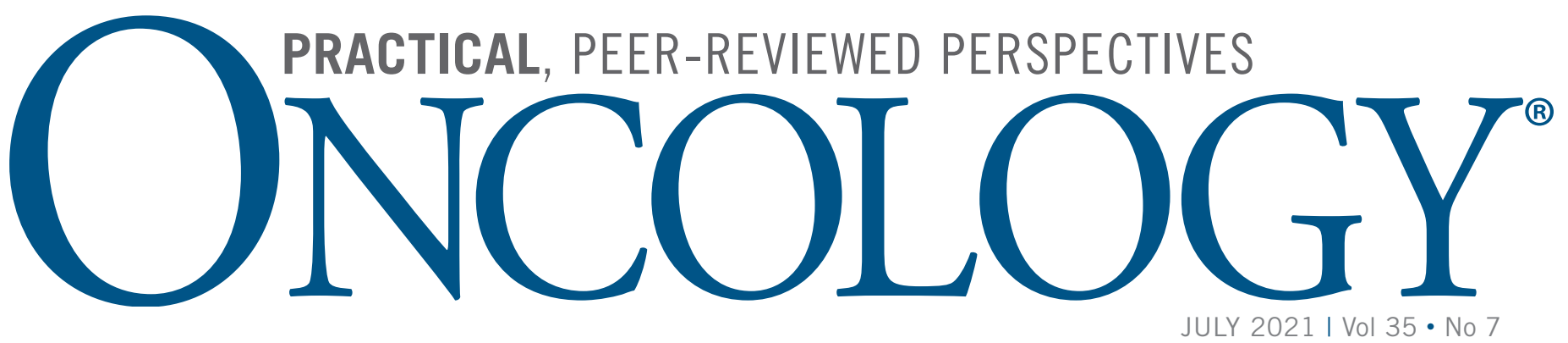

\title{
GASTROINTESTINAL CANCER
}

Pomparing GOZLIIA

and BOLOMATI

Ongoing Umbrella/Basket Trials

Examining Genetic Testing in

Gastrointestinal Malignancies

The highest level of care and expertise for your

patients with multiple myeloma.

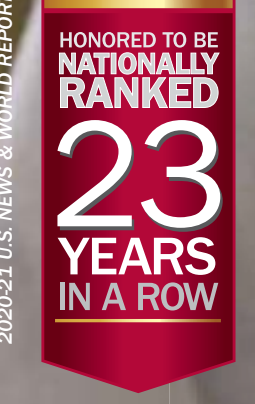

As the only specialized multiple myeloma center in the state, Indiana

University Health Medical Center is ready to partner with you.

INSIDE:

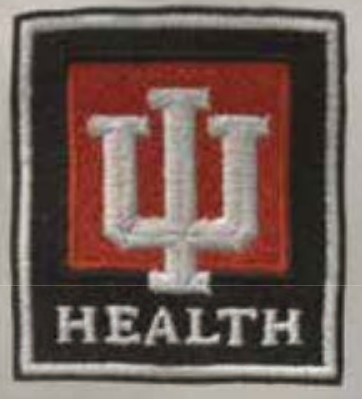

- How we're helping newly diagnosed patients achieve longerterm remissions

- Cell-based immunotherapy for multiple myeloma: A path to prevention and cures 


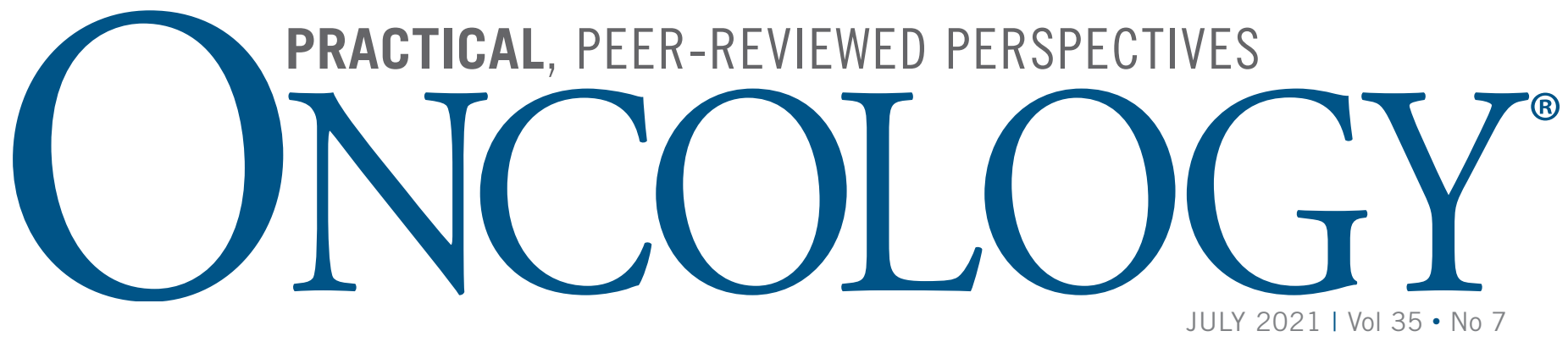

\section{GASTROINTESTINAL GANCER}

\section{BOIn paring GOZLA \\ and BOLOMATI \\ Ongoing Umbrella/Basket Trials \\ Examining Genetic Testing in \\ Gastrointestinal Malignancies}

\section{The only specialized multiple myeloma center in Indiana.}

When your patients are diagnosed with rare and complex cancers such as multiple myeloma, Indiana University Health Medical Center is your partner in the Midwest for leading-edge treatment and care.

- With the use of stem cell transplants early on in treatment of patients with multiple myeloma, our team is finding great success in helping newly diagnosed patients achieve longer-term remissions.

- Through our work with Indiana University School of Medicine, our multidisciplinary team is working to prevent and cure multiple myeloma through the use of cell-based immunotherapy.

- IU Health and Indiana University School of Medicine are actively partnering in clinical trials to advance the treatment protocols for patients with multiple myeloma.

Refer a patient at iuhealth.org/BMT 


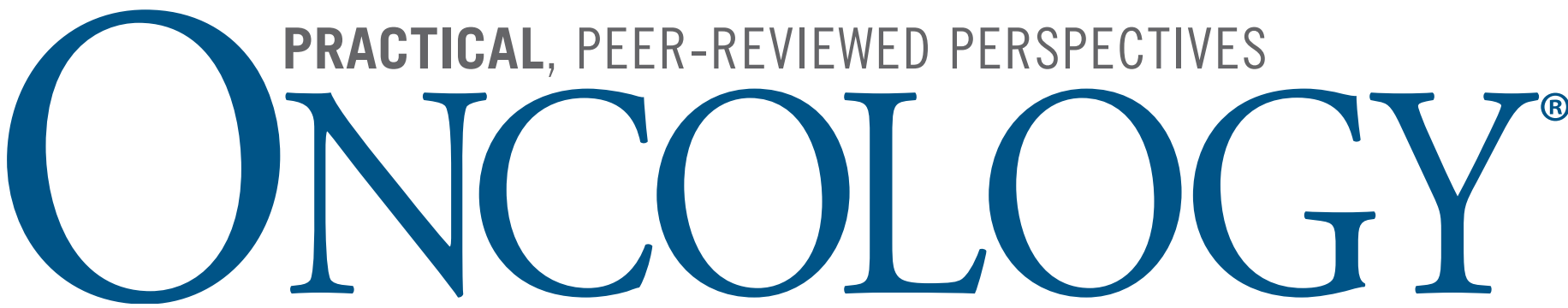

\section{GASTROINTESTINAL CANCER}

\section{BOInDaring GOZLA \\ and BOLOMATI \\ Ongoing Umbrella/Basket Trials \\ Examining Genetic Testing in \\ Gastrointestinal Malignancies}

Unmatched expertise in the treatment of multiple myeloma.

Our renowned physicians have an extensive history in establishing treatment standards for the role of cellular therapy in achieving long-term remission in patients with multiple myeloma.

\section{FEATURED PHYSICIANS}

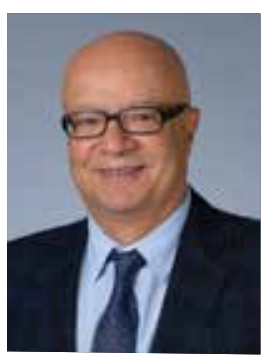

Rafat Abonour, MD Hematologist/Oncologist, Multiple Myeloma

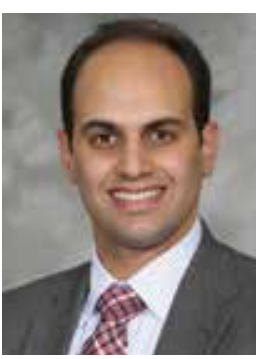

Mohammad Abu Zaid, MD Hematologist/Oncologist, Bone Marrow Transplant

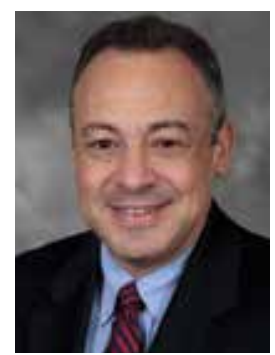

Sherif Farag, MD, PhD Hematologist/Oncologist, Bone Marrow Transplant

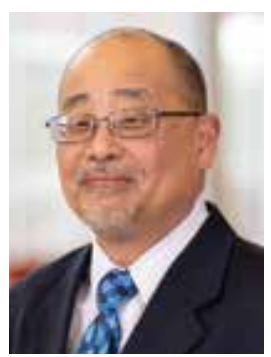

Kelvin Lee, MD Hematologist/Oncologist, Multiple Myeloma

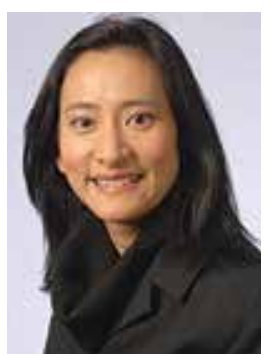

Attaya Suvannasankha, MD Hematologist/Oncologist, Multiple Myeloma 


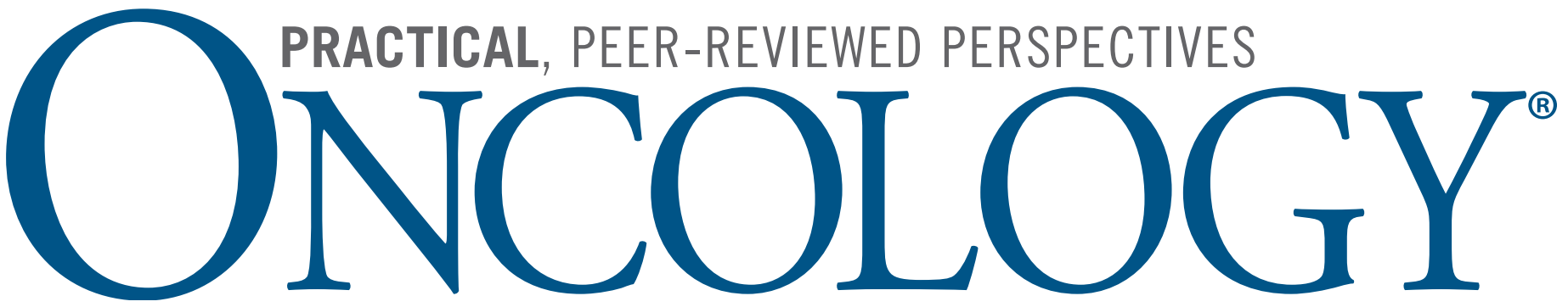

\section{GASTROINTESTINAL GANCER}

\section{Pomparing GOZLIA \\ and BOLOMATI}

Ongoing Umbrella/Basket Trials

Examining Genetic Testing in

Gastrointestinal Malignancies

\section{A nationally recognized leader in multiple myeloma treatment is always within reach.}

Multiple myeloma is a challenging disease to treat. Indiana University Health Medical Center understands this challenge-and how important it is for your patients to access the best possible treatment early in the course of the disease.

- Refer a patient: 317.944.0920 or 317.948.9401

- Contact our physician liaison, Laurita Mirkin: 463.867.9555, Imirkin@iuhealth.org

- Connect with us on Doximity

Learn more at iuhealth.org/BMT 


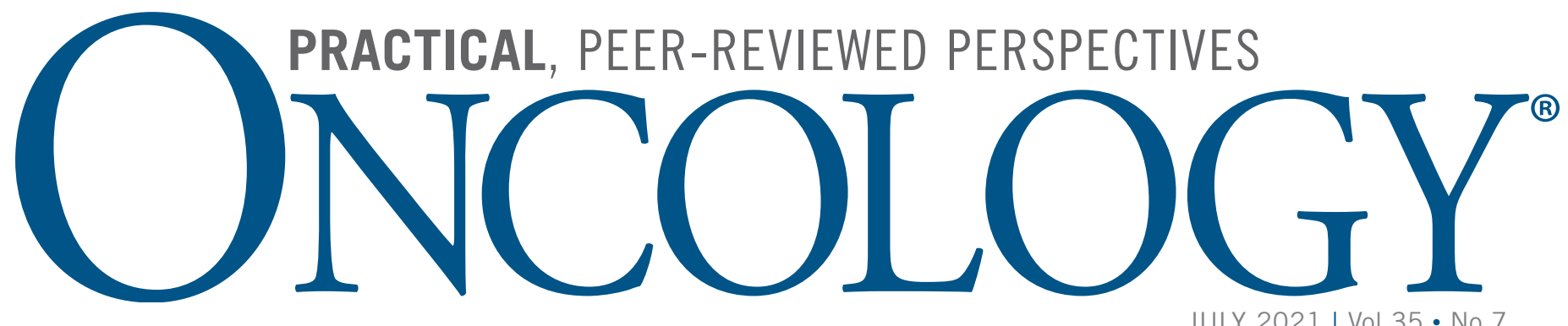

\section{GASTROINTESTINAL CANCER}

\section{POInparing GOZLIA: and BOLOMATI Ongoing Umbrella/Basket Trials Examining Genetic Testing in Gastrointestinal Malignancies}

\section{Insights From the ONCOLOGY Tumor Chairs on the 2021 ASCO Annual Meeting}

Hematologic Cancers Level of Scientific Evidence Underlying the National Comprehensive Cancer Network Clinical Practice Guidelines for Hematologic Malignancies: Are We Moving Forward?

Precision Medicine Profilin 1 Protein and Its Implications for Cancers

Urothelial Cancer Combination Therapy With Immune Checkpoint Inhibitors in Urothelial Carcinoma:

Current Data and Future Outlook

Penile Cancer Advanced Penile Cancer Presenting With Renal Failure

Immunotherapy CME The Gut Microbiome and Immunotherapy
TANIOS S. BEKAII-SAAB, MD 


\section{ARE YOU THINKING DEEP ENOUGH IN RELAPSED OR REFRACTORY MULTIPLE MYELOMA?}

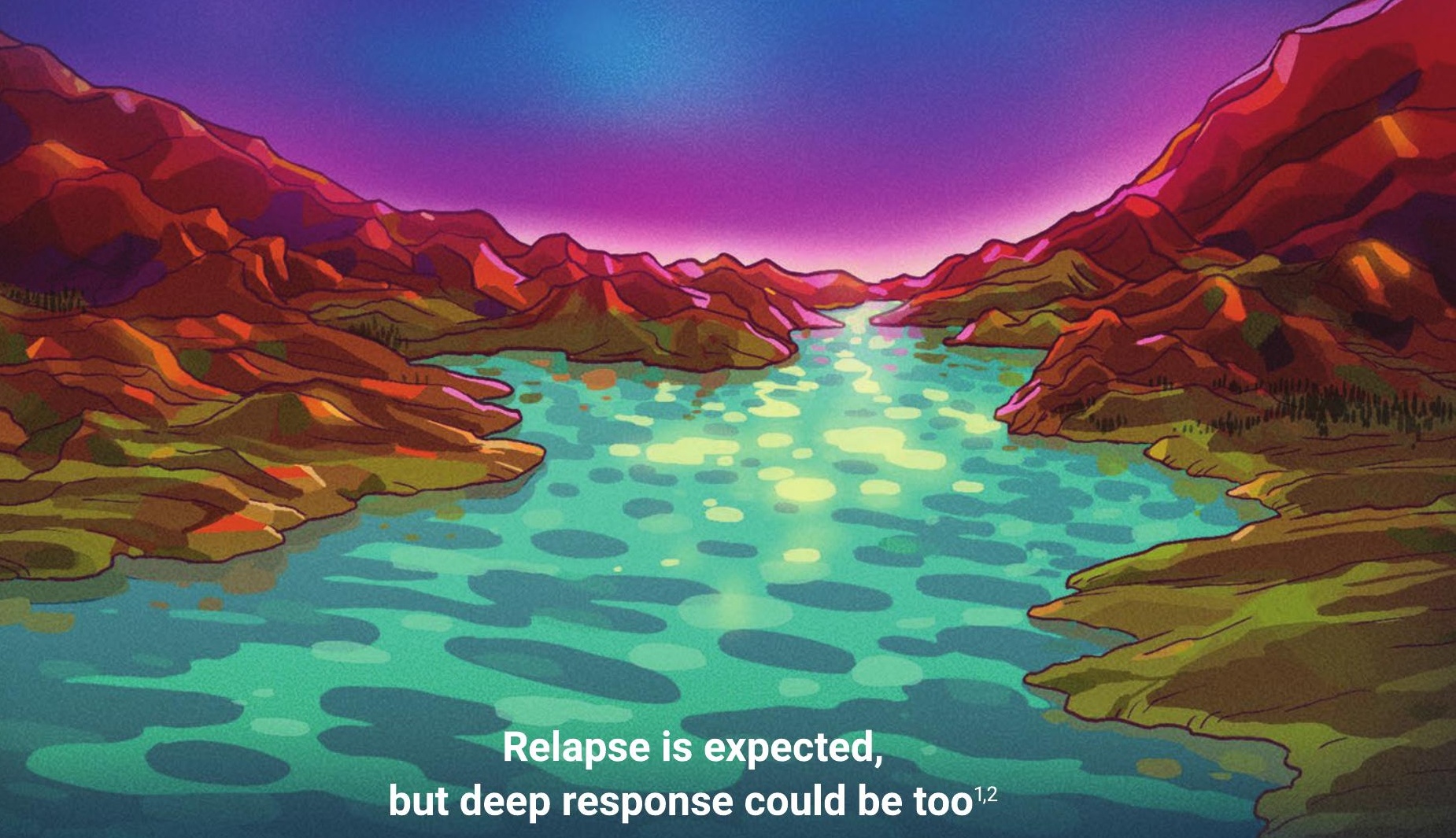

With each relapse, multiple myeloma becomes increasingly difficult to control. As the disease progresses, very few patients (less than $5 \%$ ) experience a deep response. ${ }^{1,2}$ However, evidence suggests a deep response may be associated with improved PFS and OS. ${ }^{1-3}$ Therefore, shouldn't a goal of treatment be to achieve a deep response in as many patients as possible?

The hope is that more patients may achieve a deep response with emerging therapies on the horizon.

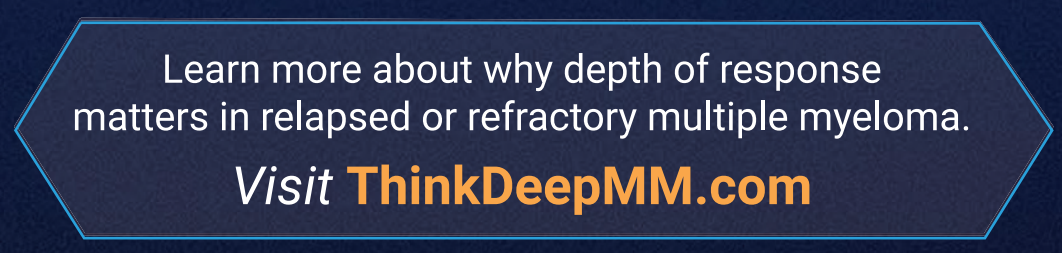

OS=overall survival; PFS=progression-free survival.

References: 1. Kumar SK, Dimopoulos MA, Kastritis E, et al. Natural history of relapsed myeloma, refractory to immunomodulatory drugs and proteasome inhibitors: a multicenter IMWG study. Leukemia. 2017;31(11):2443-2448. 2. Gandhi UH, Cornell RF, Lakshman A, et al. Outcomes of patients with multiple myeloma refractory to CD38-targeted monoclonal antibody therapy. Leukemia. 2019;33(9):2266-2275. 3. Lonial S, Anderson KC. Association of response endpoints with survival outcomes in multiple myeloma. Leukemia. 2014;28(2):258-268.

\section{Janssen $\overline{\boldsymbol{\top}}$ oncology


IN THIS ISSUE

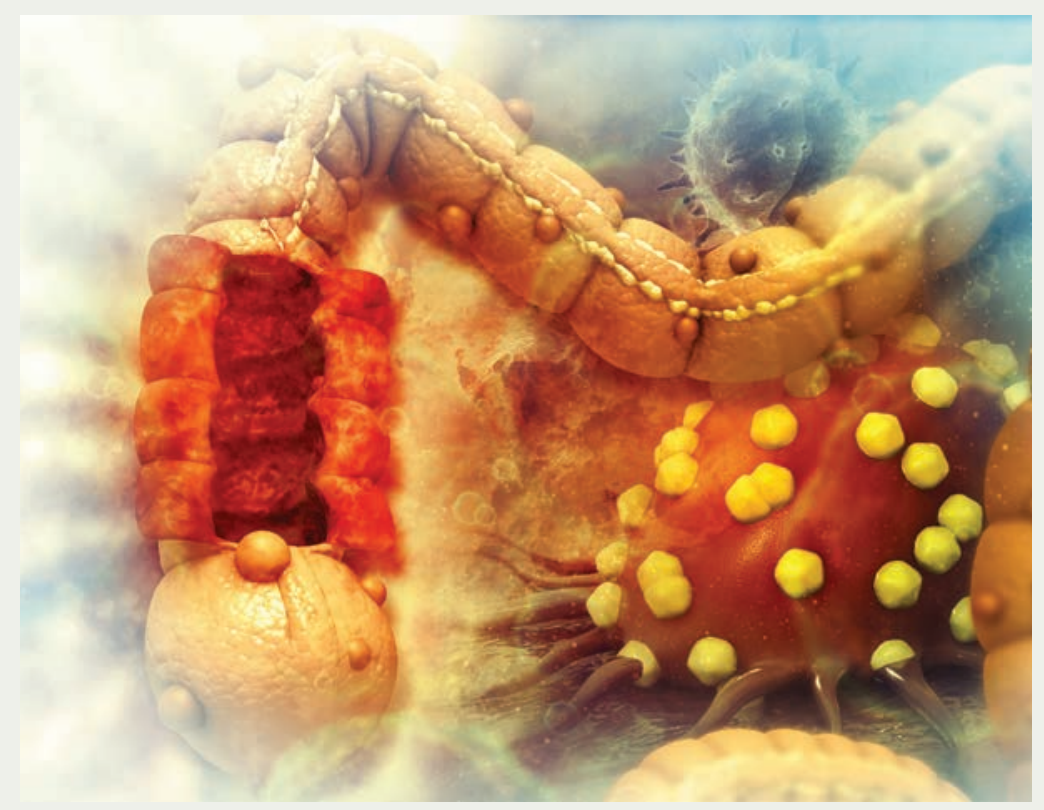

3802021 ASCO Annual Meeting

Insights From the ONCOLOGY ${ }^{\circledR}$ Tumor Chairs on the 2021 ASCO Annual Meeting

378 chairman's letter

Questions Linger Around COVID-19's Origin

Mike Hennessy Sr

379

Letter to Readers

The Future of Telehealth for Hematology/Oncology Care

Julie M. Vose, MD, MBA

\section{cancernetwork.com}

Visit CancerNetwork.com, home of the journal ONCOLOGY ${ }^{\circledR}$ and a web destination for oncologists seeking expert peer perspectives, podcasts, and other clinically practical features.

\section{VIDEO}

Suresh S. Ramalingam, MD, Discusses Adjuvant Therapy for Early NSCLC at 2021 ASCO

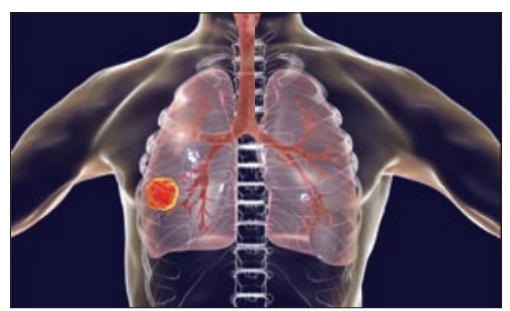

CancerNetwork.com/ASC02021_Ramalingam

\section{NEWS}

FDA Grants Priority Review to Balstilimab for Cervical Cancer After Chemotherapy

CancerNetwork.com/Balstilimab_cervical

\section{FDA APPROVAL}

Infigratinib Granted

Accelerated Approval for FGFR2+ Cholangiocarcinoma CancerNetwork.com/Sotorasib_KRASG12C

Check out our e-Newsletter for the latest in oncology.

SCAN TO SUBSCRIBE.

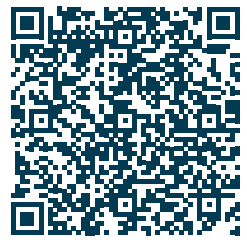


IN THIS ISSUE

GASTROINTESTINAL CANCER: COVER

382 Comparing GOZILA and COLOMATE:

Ongoing Umbrella/

Basket Trials Examining

Genetic Testing in

Gastrointestinal

Malignancies

Hideaki Bando, MD; Yoshiaki

Nakamura, MD; Daisuke Kotani, MD;

and Takayuki Yoshino, MD

GASTROINTESTINAL CANCERS:

CLINICAL TRIALS IN PROGRESS

386 GOZILA Trial

HEMATOLOGIC CANCERS: REVIEW

390 Level of Scientific

Evidence Underlying the

National Comprehensive

Cancer Network Clinical

Practice Guidelines

for Hematologic

Malignancies: Are We

Moving Forward?

Madhuri Chengappa, MBBS; Aakash P.

Desai, MBBS, MPH; Ronald S. Go, MD;

and Thejaswi K. Poonacha, MD, MBA

PRECISION MEDICINE: REVIEW

402 Profilin 1 Protein

and Its Implications

for Cancers

Ya Wang, MBBS; Yang Wang, MBBS; Rongjun Wan, MBBS; Chengping Hu, $\mathrm{MD}$; and Yichen Lu, MD

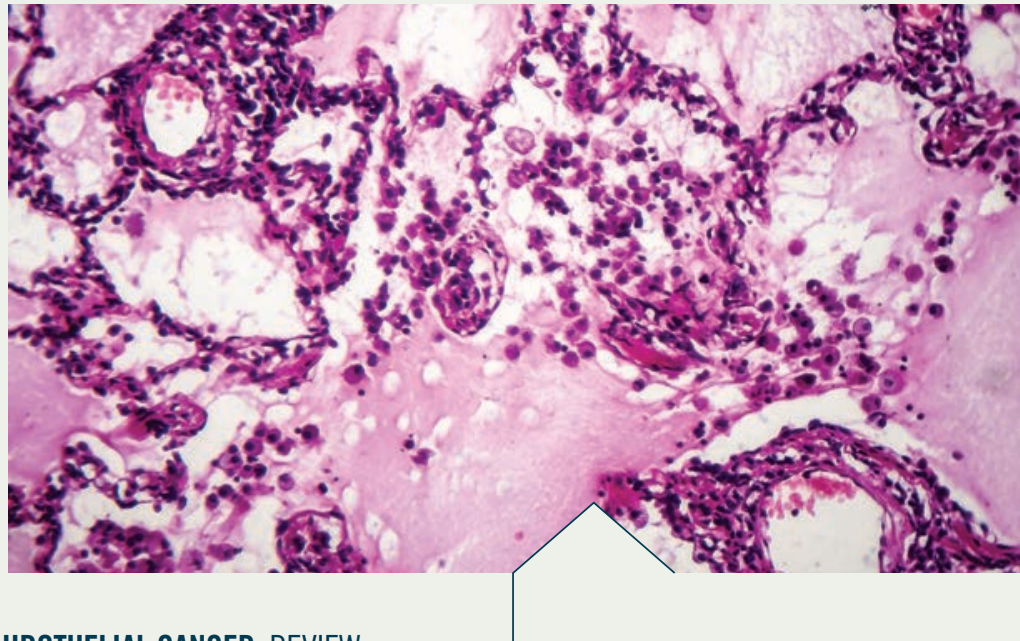

UROTHELIAL CANCER: REVIEW

410 Combination Therapy

With Immune Checkpoint

Inhibitors in Urothelial

Carcinoma: Current Data and Future Outlook

Zizhen Feng, MD, PhD; and

Jacqueline Vuky, MD

PROSTATE CANCER: CASE STUDY

422 Median Lobe Urethral

Embolus After Focal

Ablation of Gleason 7

Prostate Cancer

2021 ASCO ANNUAL MEETING: COVER

430 Rapid Reporter:

ONCOLOGY ${ }^{\circledR}$ Reviews Key

Presentations From the

2021 American Society of Clinical Oncology

Annual Meeting

IMMUNOTHERAPY:

CONTINUING MEDICAL EDUCATION

443 The Gut Microbiome and Immunotherapy

Jennifer McQuade, MD, MS, MA, LAc

Alan Makedon, BA; Paul Maroni, MD

PENILE CANCER: CLINICAL QUANDARIES

425 Advanced Penile

Cancer Presenting With

Renal Failure

Sarah Derby, MBChB; Jan Wallace,

MBChB; Balaji Venugopal; Almudena

Cascales, MSc

Published in affiliation with

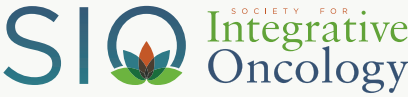

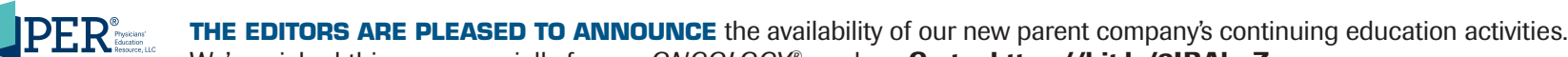
gotoper.com We've picked this one especially for our ONCOLOGY ${ }^{\circledR}$ readers. Go to: https://bit.ly/2IRAknZ guage appears within the publication: "All statements, including product claims, are those of the person or organization making the statement or claim. The publisher does not adopt any such statement or claim as its own, and any such statement or claim does not necessarily reflect the opinion of the publisher."

Advertiser and advertising agency accept and assume liability for all content (including text, representations, illustrations, opinions and facts) of advertisements printed, and also assume responsibility for any claims made against the publisher arising from or related to such advertisements. In the event that legal action or a claim is made against the publisher arising from or related to such advertisements, advertiser and advertising agency agree to fullydefend, indemnify and hold harmless the publisher, and to pay anyjudgment, expenses and legal fees incurred by the publisher as a result of said legal action or claim. The publisher reserves the right to reject any advertising which he feels is not in keeping with the publication's standards.

Publisher is not liable for delays in delivery and/or non-delivery in the event of an act of God, action by any government or quasi-governmental entity, fire, flood, insurrection, riot, explosion, embargo, strikes (whether legal or illegal), labor or materia shortage, transportation interruption of any kind, work slow-down, or any condition beyond the control of publisher affecting production or delivery in any manner.
ONCOLOGY (ISSN 0890-9091) is published monthly by MultiMedia Healthcare LLC, 2 Clarke Drive, Suite 100 Cranbury, N 08512 Annual subscription rates: US, \$237 and Canada, \$261; students and nurses, \$96; international, $\$ 296$. Single copies: $\$ 20$ each. Institutional US, $\$ 299$; Canada, $\$ 329$; international, $\$ 375$. Periodicals postage paid at Trenton, $N$ and at additional mailing offices. POSTMASTER: Please send address changes to Oncology PO Box 457, Cranbury NJ 08512-0457, USA. Publications Mail Agreement № 40612608. Return Undeliverable Canadian Addresses to: IMEX Global Solutions, PO Box 25542 London ON N6C 6B2. Canadian G.S.T number: R-124213133RT001. Printed in U.S.A.

For address changes, please notify the Circulation Department by visiting www.surveymonkey.com/s/subscriptions, or by mail to ONCOLOGY ${ }^{\circledast}$, 2021 MJH Life Sciences, PO Box 457, Cranbury NJ 08512-0457. Send old address, new address and attach a copy of mail label, if possible. 


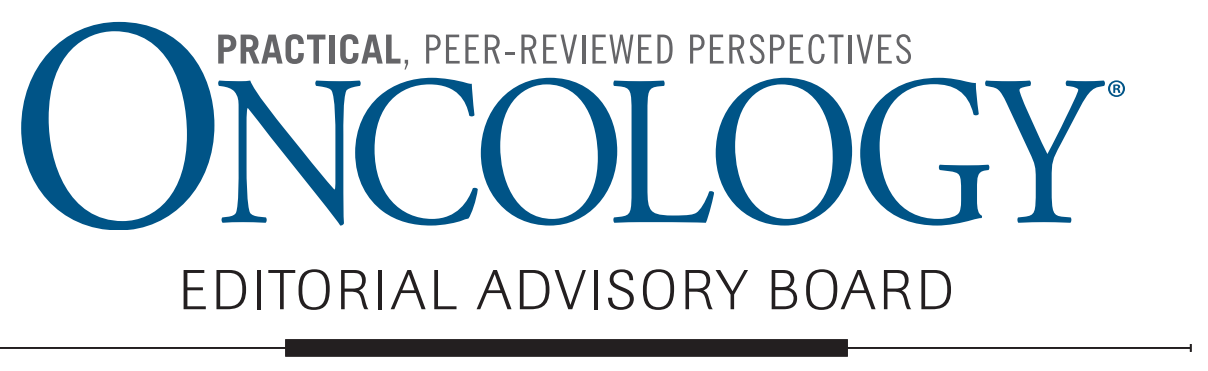

MISSION

STATEMENT

ONCOLOGY ${ }^{\circledR}$ and its website, CancerNetwork.com, provide oncologists with the practical, timely, clinical information they need to deliver the highest level of care to their patients. Expert authors and peer review ensure the quality of ONCOLOGY ${ }^{\circledR}$ and CancerNetwork.com's articles and features. Focused discussions capture key clinical take-aways for application in today's time-constrained practice environment.

\section{EDITORS-IN-CHIEF}

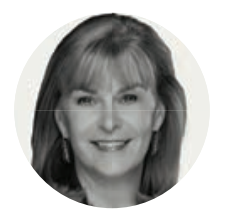

Julie M. Vose, MD, MBA Omaha, NE

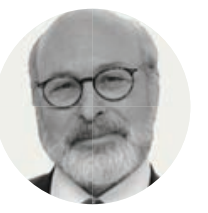

Howard S. Hochster, MD New Brunswick, NJ

\section{EDITORIAL BOARD}

\section{TUMOR CHAIRS}

BREAST CANCER

Sara A. Hurvitz, MD, Los Angeles, CA

GENITOURINARY CANCER

Robert A. Figlin, MD, Los Angeles, CA
GASTROINTESTINAL CANCER

Tanios S. Bekaii-Saab, MD, Phoenix, AZ

HEAD AND NECK CANCER

Eric J. Sherman, MD, New York, NY
HEMATOLOGIC MALIGNANCIES

C. Ola Landgren, MD, PhD, New York, NY

LUNG CANCER

Joshua M. Bauml, MD, Philadelphia, PA

\section{BOARD MEMBERS}

\section{BREAST CANCER}

William J. Gradishar, MD, FACP, Chicago, IL Tari King, MD, Boston, MA

Vered Stearns, MD, Baltimore, MD

Melinda L. Telli, MD, Palo Alto, CA

CANCER SURVIVORSHIP

Matthew J. Matasar, MD, MS, New York, NY

COLORECTAL/GASTROINTESTINAL CANCER

Edward Chu, MD, Pittsburgh, PA

Mehmet Sitki Copur, MD, FACP, Omaha, NE

Daniel Haller, MD, Philadelphia, PA

John L. Marshall, MD, Washington, DC

Shubham Pant, MD, Houston, TX

Matthew B. Yurgelun, MD, Boston, MA

Mehmet Sitki Copur, MD, FACP, Omaha, NE

GENITOURINARY CANCER

L. Michael Glodé, MD, FACP, Denver, CO

Paul Mathew, MD, Boston, MA

Elisabeth Heath, MD, FACP, Detroit, MI

Bobby Liaw, MD, New York, NY

GYNECOLOGIC ONCOLOGY

Mario M. Leitao Jr, MD, New York, NY

Franco Muggia, MD, New York, NY
HEAD AND NECK CANCER

Apar K. Ganti, MD, MS, FACP, Omaha, NE

HEALTH ECONOMICS

Nora Janjan, MD, Dallas, TX

HEMATOLOGIC MALIGNANCIES

Danielle M. Brander, MD, Durham, NC Christopher R. Flowers, MD, Houston, TX

Steven T. Rosen, MD, Duarte, CA

Naval G. Daver, MD, Houston, TX

Ehab L. Atallah, MD, Milwaukee, WI

INFECTIOUS DISEASE

Genovefa Papanicolaou, MD, New York, NY

INTEGRATIVE ONCOLOGY

Ting Bao, MD, New York, NY

Linda Carlson, PhD, RPsych, Calgary, Alberta,

Canada

LUNG CANCER

David S. Ettinger, MD, Baltimore, MD

James L. Mulshine, MD, Chicago, IL

Edward S. Kim, MD, Charlotte, NC

Jennifer W. Carlisle, MD, Atlanta, GA

MELANOMA

Richard D. Carvajal, MD, New York, NY

Jason Luke, MD, FACP, Pittsburgh, PA
NEURO-ONCOLOGY

David A. Reardon, MD, Boston, MA Stuart A. Grossman, MD, Baltimore, MD Nicole A. Shonka, MD, Omaha, NE

PEDIATRIC ONCOLOGY

David G. Poplack, MD, Houston, TX

Richard A. Drachtman, MD, New Brunswick, NJ

PROSTATE CANCER

Tomasz M. Beer, MD, Portland, OR

E. David Crawford, MD, Denver, CO

Judd W. Moul, MD, FACS, Durham, NC

PSYCHO-ONCOLOGY

Daniel C. McFarland, DO, New York, NY

Michelle Riba, MD, Ann Arbor, MI

RADIATION ONCOLOGY

Louis Potters, MD, FACR, Hempstead, NY

James B. Yu, MD, MHS, New Haven, CT

SARCOMA

Kenneth Cardona, MD, FACS, Atlanta, GA

SUPPORTIVE AND PALLIATIVE CARE

Thomas J. Smith, MD, FACP, Baltimore, MD

N. Simon Tchekmedyian, MD, Long Beach, CA

SURGICAL ONCOLOGY

Burton L. Eisenberg, MD, Newport Beach, CA

\section{INTERESTED IN SUBMITTING TO ONCOLOGY?}

Please contact Managing Editor Audrey Sternberg at ASternberg@mjhlifesciences.com for submission guidelines. 


\section{Questions Linger Around COVID-19's Origin}

Was it natural zoonotic spillover that catapulted SARS-CoV-2 from an emerging virus into a pandemic pathogen or was it something much more sinister? Is it at all possible that a lab leak in Wuhan, China, was the spark that lit the fuse?

Debate and discussion about the origin of the virus that causes COVID-19 have been at the forefront of the global consciousness since the first cases were reported in December 2019.

Early on, whispers of a potentially engineered virus quickly grew to a roar and fueled speculation that China was behind the pandemic. This narrative was so pervasive that, in February 2020, a group of 27 public health scientists published a letter in The Lancet disputing the lab leak theory and announcing their support of their counterparts in China: the scientists, public health officials, and medical professionals combating the pandemic. ${ }^{1}$

And although it's true that analyses of the genomic sequence of the virus subsequently pointed to natural origins, the questions regarding China's role persisted, led by pesky discrepancies and conflicting reports.

Fast forward to June 2021 and new evidence that has breathed new life into those origin questions. In an update to the February 2020 letter, The Lancet has published an addendum with revised disclosure statements from virologist and investigator Peter Daszak, one of the 27 authors. ${ }^{2}$ In the revised document, Daszak noted that his remuneration is paid solely in the form of a salary from EcoHealth Alliance, a New York-based nonprofit research foundation of which he is president. The company has reportedly worked directly with Wuhan laboratories and funded gain-of-function research at China's Wuhan Institute of Virology.

Consider, too, other odd associations. Recent reports have uncovered financial ties between Google and EcoHealth Alliance. This comes after accusations that the tech giant was censoring lab leak "conspiracy theory" stories in its search results. Google's health lead, David Feinberg, has dismissed those reports, insisting that the company is simply taking steps to protect users from unverified information.

We may never know where this virus came from. What we do know for certain is that the incredible strength and collaboration of the scientific community have allowed us to regain some semblance of normalcy. The development and rollout of multiple effective vaccine options have been the medical miracle of our lifetime. That, right now, will have to be the only answer that matters.

Mike Hennessy $\mathrm{Sr}$

Chairman and Founder of ONCOLOGY'S parent company, MJH Life Sciences ${ }^{\text {mp }}$

\section{REFERENCES}

1. Calisher C, Carroll D, Colwell R, et al. Statement in support of the scientists, public health professionals, and medical professionals of China combatting COVID-19. Lancet. 2020;395(10226):e42-e43. doi:10.1016/S0140-6736 (20)30418-9

2. Editors of the Lancet. Addendum: competing interests and the origins of SARS-CoV-2. Lancet. Published online June 21, 2021. doi:10.1016/S0140-6736(21)01377-5

\section{EDITORIAL}

KRISTIE L. KAHL Vice President, Content AUDREY STERNBERG Managing Editor HAYLEY VIRGIL Senior Editor MATTHEW FOWLER, ARIANA PELOSCI Assistant Editor JENNIFER POTASH Copy Chief RACHELLE LALIBERTE, PAUL SILVERMAN Copy Supervisors KELLY KING Senior Copy Editor CHENEY BALTZ, GEORGINA CARSON, KIRSTY MACKAY, RON PANAROTTI Copy Editors

\section{DESIGN \& PRODUCTION}

ROBERT MCGARR Creative Director KRISTEN MORABITO Art Director MAIA THAGARD Senior Graphic Designer JONATHAN SEVERN Circulation Director KEYONNA GRAHAM Production Director

\section{PUBLISHING \& SALES}

BRIAN HAUG Executive Vice President 609-325-4780 • bhaug@mmhgroup.com MICHELLE JANIN Director of Sales 732-429-4316•mjanin@mmhgroup.com PATRIC PASTORE National Accounts Associate 609-955-1694 • ppastore@mijlifesciences.com KRISTEN KOEDERITZ National Accounts Associate KKoederitz@mjlifesciences.com

\section{AUDIENCE DEVELOPMENT}

KELLY KEMPER Audience Development Manager

\section{CORPORATE}

MIKE HENNESSY SR Chairman \& Founder

JACK LEPPING Vice Chairman

MIKE HENNESSY JR President \& CEO

NEIL GLASSER, CPA/CFE Chief Financial Officer MICHAEL BAER Chief Marketing Officer

JOE PETROZIELLO Executive Vice President, Global Medical Affairs and Corporate Development

SILAS INMAN Senior Vice President, Content MICHAEL BALL Senior Vice President Operations SHARI LUNDENBERG Vice President, Human Resources \& Administration

CHRIS HENNESSY Vice President, Mergers \& Acquisitions JEFF BROWN Executive Creative Director. Creative Services

\section{SUBSCRIPTIONS}

888-527-7008 


\title{
LETTER TO THE READERS
}

\section{The Future of Telehealth for Hematology/Oncology Care}

\author{
Julie M. Vose, MD, MBA \\ Chief, Division of Hematology/Oncology \\ University of Nebraska Medical Center - Omaha, NE
}

$\mathrm{T}$ elehealth as a method of health care delivery has taken on greater significance due to the COVID-19 pandemic, as well as for its ability to increase access to care for those who live greater distances from their doctor's office and those unable to leave their homes. Under its section 1335 waiver authority, the Centers for Medicare \& Medicaid Services (CMS) approved temporary expansion of telehealth services during the public health emergency (PHE) to a patient's place of residence. Prior to the waiver, CMS would only pay for telehealth services on a limited basis when the patient was receiving this service in a designated rural area at a medical facility. CMS also expanded telehealth services by allowing telephonic evaluation and management services. This aspect is essential as many older or disadvantaged patients are unable to follow the instructions or lack internet access to allow telehealth video visits.

However, many of the telehealth flexibilities and policy changes made by Congress and Health and Human Services (HHS) are due to expire at the conclusion of the PHE, wherein patients and physician practices would be expected to revert to primarily face-to-face services without any type of risk-based assessment for gradually reopening medical practices and health systems to care for nonacute patients without COVID-19 infections. Patients with cancer have reported high satisfaction with telemedicine, allowing better continuity of care, enhanced communications, and greater treatment adherence, which would be a good incentive to continue the use of telehealth for this population, where appropriate.

\section{Continuation of popular telehealth services for hematology/oncology treatment will enhance care for our patients with cancer}

Prior to the PHE, there were several disincentives to the wide development of telehealth. These included difficulty securing payment from CMS for telehealth and difficulty providing telehealth services across state lines. Prior to the pandemic, many states required individual state licensure for physicians while some states participate in the Interstate Medical Licensure Compact (IMLC), which launched in 2017 and established a voluntary, expedited pathway to licensure of physicians wishing to practice in multiple states. The IMLC currently includes 29 states and the District of Columbia, and reduces the burden placed on medical providers to independently become licensed in multiple states. If all states participated in the IMLC that would break some of these barriers for the wider use of telehealth.

With hematology/oncology care facilities located in cities close to state lines or rural areas, telehealth video and telephone visits have been a great improvement for patient care. Telehealth allows patients who would otherwise need to travel long distances for a short visit to receive convenient and personalized care virtually. However, some patients who need a full physical examination; lack an established relationship with the hematology/oncology physician; or are receiving anti-neoplastic therapy and need a more thorough evaluation are more suited for in-person visits. Several bills are being considered to enhance telehealth on a more permanent basis, including the Telehealth Modernization Act (HR 1332/S 368) and the Audio-Only Telehealth Act (HR 3447/S 150). With proper safeguards, continuation of popular telehealth video and telephonic services for hematology/oncology treatment will enhance the care for our patients with cancer or blood disorders. 


\section{Insights From the ONCOLOGY ${ }^{\circledast}$ Tumor Chairs on the 2021 ASCO Annual Meeting}

T he 2021 American Society of Clinical Oncology Annual Meeting (ASCO 2021) continues to hold its place as the most impactful medical meeting for advancements in the field of cancer treatment, with pivotal presentations across a wide range of tumor types shaking up the standard of care each year.

Despite being hosted in a completely virtual format for 2 years in a row, the annual meeting provides clinicians with the opportunity to advance their knowl- edge regarding breakthroughs in the treatment of cancer, reflect on the current treatment paradigm, and collaborate with others representing an array of multidisciplinary specialties in the field of oncology.

Given the impact of these data, the editors of ONCOLOGY ${ }^{\circledR}$ sat down with some of the journal's tumor chairs to draw on their expertise and assess which presentations coming out of ASCO 2021 had the greatest potential to impact the current treatment paradigm and make way into clinical practice.

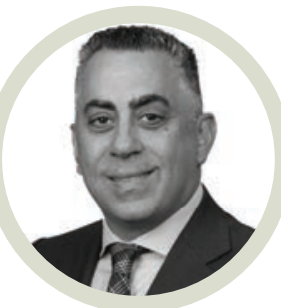

TANIOS S. BEKAII-SAAB, MD

Leader, Gastrointestinal Program

Mayo Clinic

PHOENIX, AZ
On pembrolizumab (Keytruda) in microsatellite instability-high/mismatch repair deficient (MSI-h/MMRd) metastatic colorectal cancer (mCRC)1: "We now have the results of KEYNOTE-177 [NCT02563002] in terms of the survival. [They are] very interesting, because they show that [overall] survival, although not significantly improved, was favored, meaning the hazard ratio [for pembrolizumab vs chemotherapy for MSI-h/ MMRd mCRC] was 0.74 [95\% CI, 0.53-1.03; $P=.0359$ ]. Although the median was not reached yet vs the patients who went on chemotherapy, their survival was 36.7 months. Keep in mind that a lot of these patients crossed over to pembrolizumab...I'm not waiting for survival; I'm changing my practice... because about $30 \%$ to $40 \%$ of [these] patients will never see chemotherapy in their lifetime. They're cured of their cancer. And what this update tells me is that this is going to, essentially, slightly improve survival by changing biology early and at least it will not make survival worse. The survival is actually, if anything, a little bit better with pembrolizumab first, and I think there is no excuse anymore not to say that this is the standard."
On ciltacabtagene autoleucel (cilta-cel), a chimeric antigen receptor (CAR) T-cell therapy that targets B-cell maturation antigen (BCMA), in patients with previously treated multiple myeloma ${ }^{2}$ and minimal residual disease (MRD) negativity in hematologic cancers: "The new updates for JNJ-4528 [cilta-cel] [from the] the CARTITUDE-2 trial [NCT04133636] are very exciting to see, [in] that it is a BCMA-targeted CAR T-cell [therapy] in patients with multiple myeloma who are heavily pretreated. I think [the data are] very, very strong and sends a message that cell therapy could have an important role for many patients.

If I look across the board for the blood cancers, we see better and better outcome. We have seen better outcome for many years, but it just keeps on coming. I continue to see more and more focus on MRD detection...that's what everyone is going after. And you can see deep responses, sustained MRD negativity both in newly diagnosed and relapsed [disease]. This is part of another development, and that's the moving away from old chemotherapy toward immunotherapy. The future, to me, seems to be 'bye-bye chemotherapy, welcome immunotherapy,' with a focus on sustained MRD negativity.”

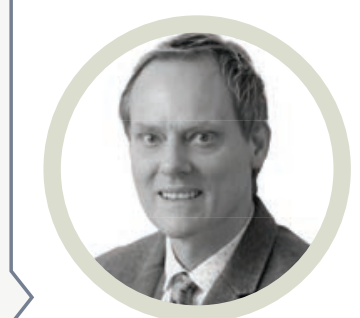

\section{OLA LANDGREN, MD, PHD}

Chief, Myeloma Program

Leader, Experimental Therapeutics Program

Sylvester Comprehensive Cancer Center

Professor of Medicine

University of Miami

MIAMI, FL 


\section{On KEYNOTE-564, evaluating pembrolizumab vs placebo as postnephrectomy adjuvant} therapy for clear cell renal cell carcinoma": "When one looks at the progression-free survival curves that were presented [at] ASCO, the curves separated very early and stayed separated over the course of the first 24 months. And what one might want to ask is this: Even though about $6 \%$ of patients had metastatic disease that was resected, was the [immuno-oncology] therapy really benefiting that population without a clear signal in the high-risk, resected nonmetastatic patient population? I think that the trial, while positive, is a bit flawed. Additionally, that could have been clarified with a better control of imaging — having blinded imaging at the beginning of the trial—-to make sure that we all agree [they were] basically investigator-assessed outcomes. And while I think that's important in general, one has to be very careful when one includes a population of patients who also have known, biopsy-proven metastatic disease. [Disease-free survival], although possibly appropriate without independent radiologic review for high-risk, resected patients [in the adjuvant setting], is generally not a form that we would take for the patients with known metastatic disease. I think [it was an] interesting trial [that is] potentially practice changing, certainly different than what we saw with targeted therapy in this setting. [But the results are] not yet in peer-reviewed publications for all of us to look at, and [the study] has some challenges that will make it a bit difficult to interpret. We're going to have to look for other adjuvant trials in a more 'pure' high-risk, resected population to assert that this is really practice changing."

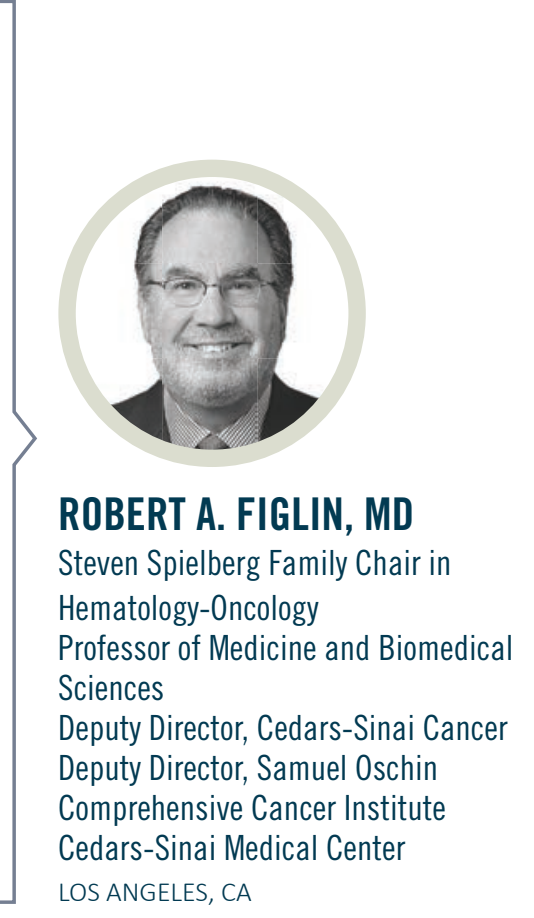

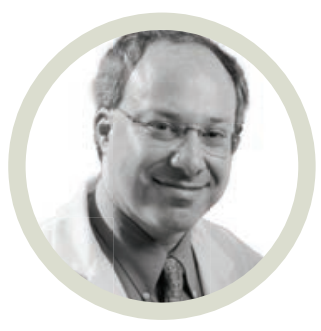

ERIC J. SHERMAN, MD

Head and Neck Oncology Service Memorial Sloan Kettering Cancer Center NEW YORK, NY
On the use of capecitabine in patients with nasopharyngeal cancer ${ }^{4,5}$ : "Two studies looked at the question of using capecitabine as adjuvant therapy for nasopharynx cancer, both looking at advanced nasopharynx cancer after cisplatin and radiation. One allowed neoadjuvant therapy, and the other did not. The one thing you saw pretty clearly is that adjuvant capecitabine led to a survival benefit-definitely a progression-free survival benefit-but it looks like an overall survival benefit, [too]. That's really important. There's a really big push away from adjuvant chemotherapy. This is showing that using a drug that can be given as a pill, not even [intravenous] treatment, and at reasonable doses, leads to a benefit independent of whether [the patient had] adjuvant therapy or not. These were not the high doses of capecitabine: One [study used] $1000 \mathrm{mg} / \mathrm{m}^{2}$ for 2 weeks on/1 week off, and the other study used just $650 \mathrm{mg} / \mathrm{m}^{2}$ twice a day, continued for a full year...This is something that may be a real [practice] changer in how we treat."

\section{References}

1. Andre T, Shiu K-K, Kim TW, et al. Final overall survival for the phase III KN177 study: pembrolizumab versus chemotherapy in microsatellite instability-high/mismatch repair deficient (MSI-H/dMMR) metastatic colorectal cancer (mCRC). J Clin Oncol. 2021;39(suppl 15):3500. doi:10.1200/JCO.2021.39.15_suppl.3500

2. Agha ME, Cohen AD, Madduri D, et al. CARTITUDE-2: efficacy and safety of ciltacabtagene autoleucel (cilta-cel), a BCMA-directed CAR T-cell therapy, in patients with progressive multiple myeloma (MM) after one to three prior lines of therapy. J Clin Oncol. 2021;39(suppl 15):8013. doi:10.1200/JC0.2021.39.15_suppl.8013

3. Choueiri TK, Tomczak P, Park SH, et al. Pembrolizumab vs placebo as post-nephrectomy adjuvant therapy for patients with renal cell carcinoma: randomized, double-blind, phase III KEYNOTE-564 study. J Clin Oncol. 2021;39(suppl 15):LBA5. doi:10.1200/JC0.2021.39.15_suppl.LBA5

4. Miao J, Wang L, Tan SH, et al. Adjuvant capecitabine in locoregionally advanced nasopharyngeal carcinoma: a multicenter randomized controlled phase III trial. J Clin Oncol. 2021;39(suppl 15):6005. doi:10.1200/ JC0.2021.39.15_suppl.6005

5. Ma J, Chen Y-P, Sun Y, et al. Metronomic capecitabine as adjuvant therapy in locoregionally advanced nasopharyngeal carcinoma: a phase 3, multicenter, randomized controlled trial. J Clin Oncol. 2021;39(suppl 15):6003. doi:10.1200/JCO.2021.39.15_suppl.6003 


\section{Comparing GOZILA and COLOMATE: Ongoing Umbrella/Basket Trials Examining Genetic Testing in Gastrointestinal Malignancies}

Hideaki Bando, MD*; Yoshiaki Nakamura, MD*; Daisuke Kotani, MD; and Takayuki Yoshino, MD

ABSTRACT: Circulating tumor DNA (ctDNA)-based nextgeneration sequencing (NGS) assays have advantages over classic tissue-based analyses because of their low invasiveness and availability of repeated sampling. Because of the low incidence of target gene alterations such as HER2 or BRAF V600E in gastrointestinal cancers, very large screening platforms are needed to develop genome-based clinical trials. For those reasons, ctDNAbased screening studies are being actively conducted; among them are the GOZILA (Guardant Originates in Zipangu Liquid biopsy Arrival) study in Japan and the COLOMATE (COlorectal Cancer Liquid BiOpsy Screening Protocol for Molecularly Assigned ThErapy) study in the United States.

Although only patients with metastatic colorectal cancer (mCRC) who had previously received standard chemotherapies are eligible for the COLOMATE study, patients with various types of solid tumors at any line of treatment are eligible for GOZILA. This broad coverage of the eligible population allows a target of 5000 patients. By contrast, effective screening of selected candidate patients for companion trials using rapid turnaround time by ctDNA-based NGS assay is the key for COLOMATE. The companion trials of targeted therapies in $\mathrm{mCRC}$ are similar between GOZILA and COLOMATE. Both studies have identified patients eligible for studies by examining ERBB2 (HER2), BRAF V600E, BRAF non-V600E, and FGFR alterations, as well as MET amplification and rechallenge with anti-EGFR antibodies. The existence of various companion trials for common alterations that can be potential therapy targets on the 2 platforms can lead to future international collaboration.

KEY WORDS: colorectal cancer; circulating tumor DNA; GOZILA; COLOMATE; companion trials; targeted therapy

\section{Introduction}

Colorectal cancer (CRC) is the third most common cancer in men and the second most common cancer in women worldwide as of 2020. ${ }^{1}$ Although several cytotoxic drugs and targeted therapies improve the survival of patients with unresectable disease, median survival is only 30 months. $^{2}$ Therefore, additional treatment strategies are needed for patients with advanced disease. Prospective studies based on genomic targets such as ERBB2 (HER2), BRAF V600E, and microsatellite instability (MSI) have demonstrated clinical benefits for patients with metastatic CRC (mCRC).

Cell-free DNA (cfDNA) refers to extracellular DNA molecules, originating from both normal and tumor cells, that are found in body fluids. ${ }^{3}$ The tumor-derived fraction of cfDNA is commonly called circulating tumor DNA (ctDNA). Analysis of ctDNA has been shown to have huge clinical utility in detecting genetic alterations with minimal invasiveness in various types of cancers, including CRC. ${ }^{4}$ Additionally, the rapid progress of next-generation sequencing (NGS) technologies for ctDNA analyses have led to significant reductions in sequencing costs, with improved sensitivity and accuracy.

Although tissue-based genotyping is still the standard method for precision oncology, the emerging technology of ctDNA-based genotyping is gradually replacing tissue-based methods in clinical practice. ${ }^{5}$ In Japan, polymerase chain reaction-based ctDNA assays for EGFR mutations in 


\section{FIGURE 1. Overview of the SCRUM-Japan Platform for Metastatic and/or
Unresectable Cancers}

\author{
SCRUM-Japan GI-SCREEN (since February 2015) \\ Metastatic and/or unresectable \\ gastrointestinal cancers \\ Enrolled patients: 5740 \\ Screening study using tissue-based \\ next generation sequencing assay \\ (Oncomine ${ }^{\mathrm{TM}}$ Comprehensive Assay)
}

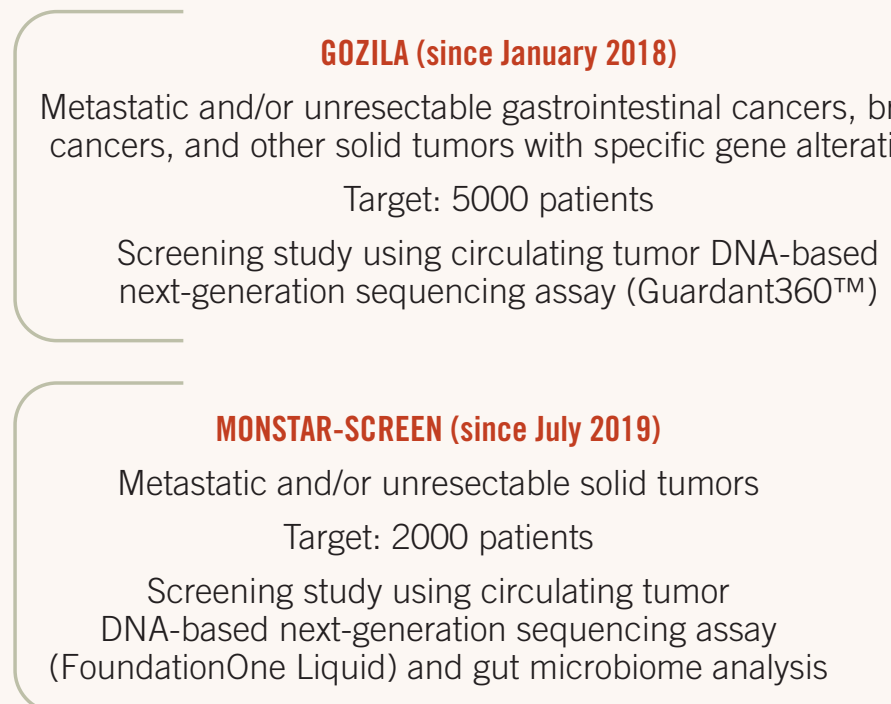

non-small cell lung cancer and RAS mutations in CRC have been approved and implemented in clinical practice. Moreover, as NGS-based methods have enabled ctDNA profiling with high sensitivity and accuracy and have confirmed analytical validities, several ctDNA-based NGS assays have been approved in both the United States and Japan.,7

ctDNA-based NGS assays have various advantages as screening tools for targeted therapies because of their low invasiveness and the availability of repeated sampling. In addition, ctDNA-based assays can detect real-time and spatial genetic status that considers both clonal evolution and tumor heterogeneity. In this review, we explain 2 studies of ctDNA-based screening platforms: GOZILA (Guardant Originates in Zipangu Liquid biopsy Arrival) in Japan (UMIN000029315) and COLOMATE (COlorectal Cancer Liquid BiOpsy Screening Protocol for Molecularly Assigned ThErapy) in the United States (NCT03765736). We also discuss the differences and similarities between the 2 platforms.

\section{SCRUM-Japan GOZILA}

To promote genome-based clinical trials in Japan, a nationwide genome screening consortium for lung cancer, the LCSCRUM-Japan, was launched in February 2013. The LC-SCRUM-Japan originally aimed to identify patients harboring ROS1 and RET fusions originally discovered in Japan. ${ }^{8}$ In February 2014, the GI-SCREEN-Japan multicenter screening project also started for gastrointestinal (GI) cancer. The project began to screen for BRAF, NRAS, and PIK3CA mutations in patients with mCRC. ${ }^{9}$ In February 2015, these 2 groups merged and initiated the SCRUM-Japan project as a collaboration of industry, government, and academia to achieve nationwide cancer genome screening for precision medicine in Japan (UMIN000016343; UMIN000016344). ${ }^{10}$ From February 2015 to June 2019, 5740 patients with GI cancer were investigated using the tissue-based Oncomine Comprehensive Assay (Thermo Fisher Scientific, Waltham, MA, USA). Subsequently, in January 2018, we initiated GOZILA, a screening study based on the SCRUM-Japan platform using comprehensive ctDNA sequencing by Guardant $360^{\mathrm{TM}}$ (Guardant Health, Redwood City, CA, USA) to rapidly screen patients for trial eligibility. ${ }^{11,12}$
Further, in July 2019, we started MONSTAR-SCREEN (UMIN000036749), a multi-omics profiling and monitoring study using ctDNA sequencing by FoundationOne Liquid (Foundation Medicine, Cambridge, MA, USA) and gut microbiome analysis (Figure 1).

Patients are eligible for GOZILA if they have metastatic and/or unresectable GI cancers, including CRC, gastroesophageal adenocarcinoma (GEA), esophageal squamous cell carcinoma (ESCC), pancreatic ductal adenocarcinoma (PDAC), and cholangiocarcinoma (CCA). Additionally, patients with metastatic and/or unresectable breast cancer and other solid tumors with specific gene alterations diagnosed by quality-assured tissue-based in vitro diagnostic tests are eligible for GOZILA.

Compared with the tissue-based GISCREEN project, GOZILA has a significantly shorter sample acquisition duration (median, 4 days vs 14 days; $P<.0001$ ) and shorter test duration (median, 7 days vs 19 days; $P<.0001)$. Based on the increased screening speed, GOZILA significantly increased the relative proportion of patients enrolled in clinical trials for targeted therapies $(9.5 \%$ vs $4.1 \% ; P<.0001) .{ }^{12}$ 
TABLE 1. Related Clinical Trials in GOZILA Study

\begin{tabular}{|c|c|c|c|c|c|}
\hline $\begin{array}{l}\text { Trial identifier } \\
\text { (title acronym) }\end{array}$ & Phase & $\begin{array}{l}\text { Estimated \# } \\
\text { of patients }\end{array}$ & Criteria for patient selection & Intervention & $\begin{array}{l}\text { Primary } \\
\text { end point }\end{array}$ \\
\hline \multicolumn{6}{|c|}{ Umbrella trials for mCRC } \\
\hline $\begin{array}{l}\text { UMIN000027887 } \\
\text { (TRIUMPH) }\end{array}$ & 2 & 30 & $\begin{array}{l}\text { Tissue or ctDNA RAS wild-type and } \\
\text { HER2 amplification }\end{array}$ & Pertuzumab and trastuzumab & ORR \\
\hline $\begin{array}{l}\text { UMIN000031221 } \\
\text { (BRAVERY) }\end{array}$ & 2 & 42 & $\begin{array}{l}\text { Tissue or ctDNA BRAF V600E } \\
\text { mutant }\end{array}$ & Eribulin & ORR \\
\hline $\begin{array}{l}\text { UMIN000031857 } \\
\text { (BIGBANG) }\end{array}$ & 2 & 36 & $\begin{array}{l}\text { Tissue or ctDNA BRAF non-V600E } \\
\text { mutant }\end{array}$ & $\begin{array}{l}\text { Encorafenib, binimetinib, and } \\
\text { cetuximab }\end{array}$ & ORR \\
\hline $\begin{array}{l}\text { JapicCTI-194677 } \\
\text { (METBEIGE) }\end{array}$ & $\begin{array}{l}1 / 2 \\
\text { (randomized) }\end{array}$ & 54 & ctDNA MET amplification & $\begin{array}{l}\text { Cabozantinib monotherapy or } \\
\text { cabozantinib plus panitumumab }\end{array}$ & ORR \\
\hline $\begin{array}{l}\text { jRCTs031190096 } \\
\text { (PURSUIT) }\end{array}$ & 2 & 50 & $\begin{array}{l}R A S \text { wild-type with previous } \\
\text { response to anti-EGFR antibody }\end{array}$ & Panitumumab and irinotecan & ORR \\
\hline \multicolumn{6}{|c|}{ Umbrella trial for unresectable CCA } \\
\hline $\begin{array}{l}\text { JMA-IIA00423 } \\
\text { (HERB) }\end{array}$ & 2 & 32 & $\begin{array}{l}\text { Tissue HER2 positivity (ctDNA test } \\
\text { was added to evaluate } H E R 2 \text { ampli- } \\
\text { fication and other gene alterations) }\end{array}$ & Trastuzumab-deruxtecan & ORR \\
\hline \multicolumn{6}{|c|}{ Basket trials for patients with solid tumors } \\
\hline $\begin{array}{l}\text { UMIN000033182 } \\
\text { (bTMB-H basket) }\end{array}$ & 2 & 70 & High blood-based TMB & Nivolumab & ORR \\
\hline $\begin{array}{l}\text { JapicCTI-194624 } \\
\text { (TIFFANY) }\end{array}$ & 2 & 26 & ctDNA FGFR alterations & Futibatinib & ORR \\
\hline $\begin{array}{l}\text { JapicCTI-194707 } \\
\text { (HERALD) }\end{array}$ & 2 & 60 & ctDNA HER2 amplification & Trastuzumab-deruxtecan & ORR \\
\hline $\begin{array}{l}\text { JapicCTI-194851 } \\
\text { (Barossa) }\end{array}$ & 2 & 52 & Tissue or ctDNA ROS1 fusion & Brigatinib & ORR \\
\hline $\begin{array}{l}\text { jRCT2011200023 } \\
\text { (NIR-B) }\end{array}$ & 2 & 60 & $\begin{array}{l}\text { Tissue or ctDNA BRCA mutation } \\
\text { (only for patients with GI cancers) }\end{array}$ & Niraparib & ORR \\
\hline
\end{tabular}

CCA, cholangiocarcinoma; ctDNA, circulating tumor DNA; GI, gastrointestinal; mCRC, metastatic colorectal cancer; ORR, objective response rate; $\mathrm{TMB}$, tumor mutational burden.

However, when we compared the efficacy between GI-SCREEN and GOZILA, patients demonstrated similar objective response rates $(20.0 \%$ vs $16.7 \%)$ and progression-free survival (PFS; median, 2.4 months vs 2.8 months). ${ }^{12}$

We have also reported the genomic profiles of GI cancers based on ctDNA-based NGS data from the GOZILA study. Patients with ESCC and CRC had the highest ctDNA detection rates $(99.1 \%$ and $96.0 \%$, respectively), whereas patients with PDAC and GEA had lower detection rates $(83.4 \%$ and $85.3 \%$, respectively). We also detected multiple biomarkers relevant to therapy selection, including KRAS, NRAS, BRAF, and PIK3CA mutations; ERBB2 (HER2), FGFR1/2, and MET amplifications; FGFR2/3, ALK,
NTRK1, and RET fusions; and MSI. In addition, both germline and somatic $B R C A$ mutations were reported in ctDNA samples from patients with PDAC and CCA. ${ }^{12}$

GOZILA is thus a large and comprehensive ctDNA-based screening platform for GI cancers and other malignancies. GOZILA also significantly shortened the screening turnaround time and improved the trial enrollment rate without compromising treatment efficacy compared with tissue-based screening.

\section{GOZILA-related clinical trials}

Various types of prospective umbrella/ basket clinical trials have been conducted based on this comprehensive GOZILA platform. GOZILA has identified patients for various types of organ-specific umbrella trials for mCRC (Table 1; see Figure 2 on page 387).

HER 2 amplification occurs in $1 \%$ to $4 \%$ of patients with mCRC, ${ }^{13-16}$ and 2 phase 2 trials, HERACLES (NCT03225937) and MyPathway (NCT02091141), showed the efficacy of HER2-targeted therapies. ${ }^{17,18}$ Therefore, we initiated a single-arm phase 2 trial to evaluate the efficacy of pertuzumab (Perjeta) plus trastuzumab (Herceptin) in patients with mCRC with HER2 amplification that had been confirmed by tumor tissue or ctDNA analysis (TRIUMPH; UMIN000027887). A HER2 positivity rate of $5 \%$ to $20 \%$ has also been reported among patients with CCA. Trastuzumab deruxtecan (Enhertu) is an antibody-drug 
conjugate consisting of trastuzumab, a cleavable tetrapeptide-based linker, and a cytotoxic topoisomerase I. Trastuzumab deruxtecan is approved for patients with HER2-positive metastatic gastric and breast cancer in both Japan and the United States. We initiated a single-arm phase 2 trial that investigated the efficacy and safety of trastuzumab deruxtecan in patients with unresectable HER2-positive CCA (HERB; JMA-IIA00423). Although the HER 2 status was diagnosed by immunohistochemistry and/or in situ hybridization, the ctDNA test was added to evaluate HER2 amplification and other gene alterations using the GOZILA platform.

$B R A F$ V600E mutation also occurs in $5 \%$ to $10 \%$ of $\mathrm{mCRCs}^{19-22}$ and is an established factor of poor prognosis. Although the results of BEACON CRC (NCT02928224) indicated that combination therapy with anti-BRAF (encorafenib; Braftovi), anti-MEK (binimetinib; Mektovi), and antiEGFR (cetuximab; Erbitux) agents led to a significant improvement in survival compared with standard irinotecan-based therapy, ${ }^{23,24}$ further development of new treatments is very much needed. Because the protein RANBP2 increases microtubule outgrowth from the kinetochores and the protein shRANBP2 impairs proliferation of BRAF V600E-mutant CRC cell lines, ${ }^{25}$ we conducted a single-arm phase 2 trial to investigate the efficacy and safety of eribulin, a microtubule inhibitor, in patients with $B R A F$ V600E mutations (BRAVERY; UMIN000031221). ${ }^{26}$ In addition, because BRAF mutations other than V600E (BRAF non-V600E) have been observed in $1.6 \%$ to $5.1 \%$ of $\mathrm{mCRCs}^{27-31}$ and are increasingly identified by NGS-based assays in patients with mCRC, we initiated a single-arm phase 2 trial to investigate the efficacy and safety of encorafenib, binimetinib, and cetuximab in patients with BRAF non-V600E mutations (BIGBANG; UMIN000031857). ${ }^{32}$

Further, a longitudinal surveillance of ctDNA by NGS-based assays during
anti-EGFR therapy indicated the emergence of acquired RAS mutations and alterations in other genes, including MET, ERBB2 (HER2), FLT3, EGFR, and $M E K{ }^{33,34}$ We initiated a randomized phase 2 trial to investigate cabozantinib (Cabometyx) monotherapy vs cabozantinib plus panitumumab (Vectibix) in patients with both primary and acquired MET amplification (METBEIGE; JapicCTI-194709) and a single-arm phase 2 trial to investigate panitumumab plus irinotecan rechallenge in patients with $R A S$ wild-type disease who developed resistance to anti-EGFR antibodies (PURSUIT; jRCTs031190096).

Additionally, GOZILA has several ongoing basket studies to test tissue-agnostic approaches (Figure 2, Table 1).

Tumor mutational burden (TMB) has been established as a predictive biomarker for the efficacy of immune checkpoint inhibitors, ${ }^{35,36}$ but no prospective clinical trials have investigated the clinical utility of blood-based TMB (bTMB) tests. We are currently investigating the efficacy of nivolumab for treating bTMB-

\section{The existence of various companion trials for the same target alteration can lead to future international collaboration with the use of [GOZILA and COLOMATE].}

high solid tumors (bTMB-H basket; UMIN000033182). FGFR alterations, including amplifications, mutations, rearrangements, and fusions, are observed in many types of cancer, and pemigatinib (Pemazyre) and infigratinib (Truseltiq) were granted accelerated approval by the FDA for patients with previously treated, unresectable, or metastatic CCA with FGFR2 fusions or other rearrangements. We are currently investigating the efficacy and safety of futibatinib, a pan-FGFR inhibitor, in patients with solid tumors with FGFR alterations (TiFFANY; JapicCTI-194624). In addition to the HERB trial for unresectable CCA, we have investigated the efficacy and safety of trastuzumab deruxtecan for HER2-amplified solid tumors, excluding GEA, breast cancer, lung cancer, CCA, uterine carcinosarcoma, and osteosarcoma, using the ctDNA-based GOZILA platform (HERALD, JapicCTI-194707). Also, for other alterations with tissue-agnostic distributions, we have initiated investigator-initiated studies, including of brigatinib (Alunbrug) for solid tumors with ROS1 fusions (Barossa; JapicCTI-194851) and of niraparib for GI cancers with $B R C A$ mutations (NIR-B; jRCT2011200023).

The results of the TRIUMPH (NCT03413995) and BRAVERY (UMIN000031221) studies have been reported. In the TRIUMPH study, pertuzumab and trastuzumab were administered every 3 weeks to patients with tissue or ctDNA RAS wild-type and HER2-amplified mCRC. ${ }^{37}$ The HER 2 amplification agreement between the tissue and ctDNA was $83 \%(62 / 75)$. Overall response rate (ORR) was the primary end point, and ORRs of $30 \%$ (95\% CI, $14 \%-50 \%$ ) in 27 tissue-positive patients and of $28 \%(95 \%$ CI, $12 \%$ $49 \%$ ) in 25 ctDNA-positive patients were observed. While 1 partial response (PR), 3 stable disease (SD), and 1 progressive disease (PD) were observed in 5 patients with ctDNA-negative/tissue-positive tumors, only 1 SD and 2 PD were observed in 3 patients with ctDNA-positive/tissue-negative tumors. The confirmed median PFS and median overall survival (OS) were 4.0 months (95\% CI, 1.4-5.6) and 10.1 months (95\% CI, 4.5-16.5) in tissue-positive patients, respectively, and 3.1 months (95\% CI, 1.4-5.6) and 8.8 months $(95 \%$ CI, 4.3-12.9) in ctDNA-positive patients, respectively. In exploratory analyses, patients with wild-type RAS, BRAF,PIK3CA, and HER 2 were more likely to respond to 


\title{
CLINICAL TRIALS IN PROGRESS
}

\author{
GOZILA
}

\section{TITLE}

Nationwide Circulating-Tumor DNA-Guided Basket and Umbrella Clinical Trials for Patients With Advanced Solid Tumors (GOZILA; UMIN000029315)

\section{BACKGROUND}

Marked advances in precision oncology have made genotyping mandatory for most patients with advanced cancer to ensure proper therapy selection. However, tissue-based genotyping hampers patient recruitment due to the long turnaround time, and it often fails to detect chronological tumor evolution and intratumoral genomic heterogeneity. Both of these are obstacles for accurate treatment selection.

To address these challenges, we initiated a nationwide plasma-based screening project, GOZILA, in which comprehensive circulating tumor DNA (ctDNA) sequencing was used to rapidly screen patients with advanced solid tumors for trial eligibility by identifying genomic alterations in tumor cells throughout the body. Using data from the GOZILA study, we demonstrated that ctDNA genotyping led to markedly faster turnaround time and an accelerated enrollment in targeted trials while maintaining efficacy equivalent with that of tissue-based genotyping. ${ }^{1}$

Here, we are conducting basket and umbrellatype investigator-initiated, single-arm phase 2 trials based on genomic alterations identified in the GOZILA study (Figure 2). Our goal is to determine if ctDNA genotyping can identify patients who benefit from targeted therapy. In addition, we will evaluate the efficacy of targeted therapies in GOZILA-affiliated trials compared with standard-of-care therapy in SCRUM-Japan Registry, a cohort study collecting regulatorygrade, real-world data.

\section{INCLUSION CRITERIA}

The key eligibility criteria included the following: (1) histopathologically confirmed metastatic gastrointestinal cancer or another advanced solid tumor with specific genomic alterations; (2) 20 years or older; and (3) life expectancy of at least 12 weeks. To avoid the suppression of ctDNA shedding due to chemotherapy, patients were included only if they showed disease progression during systemic chemotherapy and had not started the subsequent therapy at the time of blood sampling.

Eleven investigator-initiated trials are affiliated with the GOZILA study. Patients with a specific cancer type (tumor-specific) or any solid tumors (tumor-agnostic) and with a targeted genomic alteration identified in the GOZILA study are eligible in these trials.

\section{PATIENT ACCRUAL INFORMATION}

- Open date: Open to enrollment at National Cancer Center Hospital East in January 2018.

- Accrual goal: 5000

- Percent accrued: $81 \%$ accrual completed. Among affiliated clinical trials, patient accrual has been completed in TRIUMPH, BRAVERY, HERB, and TiFFANY.

\section{STUDY SITES}

A total of 31 sites are open to accrual: National Cancer Center Hospital East, Aichi Cancer Center Hospital, National Cancer Center Hospital, National Hospital Organization Kyushu Cancer Center, Hokkaido University Hospital, Saitama Cancer Center, Kanagawa Cancer Center, Kansai Rosai Hospital, National Hospital Organization Shikoku Cancer Center, National Hospital
Organization Osaka National Hospital, University of Tsukuba Hospital, Chiba Cancer Center, Kyorin University Hospital, Kindai University Hospital, Kyushu University, St Marianna University School of Medicine, Osaka University, Cancer Institute Hospital of Japanese Foundation for Cancer Research; Kobe City Medical Center General Hospital, Osaka Medical College Hospital, Gifu University, Kanazawa University, Shizuoka Cancer Center, Kagawa University Hospital, Keio University Hospital, Saitama Medical University International Medical Center, Shimane Prefectural Central Hospital, Kansai Medical University Hospital, Kyoto Katsura Hospital, Osaka International Cancer Institute, Osama General Medical Center

\section{REFERENCE}

1. Nakamura Y, Taniguchi H, Ikeda M, et al. Clinical utility of circulating tumor DNA sequencing in advanced gastrointestinal cancer: SCRUM-Japan GI-SCREEN and GOZILA studies. Nat Med. 2020;26(12):1859-1864. doi:10.1038/s41591-0201063-5.

\section{WANT TO READ MORE ON THIS TOPIC?}

ONCOLOGY® ${ }^{\circledR}$ will be publishing more content related to genomic testing in gastrointestinal malignancies from the COLOMATE and GOZILA investigators in upcoming

issues of the journal.

\section{CONTACT INFORMATION: COPRINCIPAL INVESTIGATORS}

Yoshiaki Nakamura, MD, PhD

National Cancer Center Hospital East, 6-5-1 Kashiwanoha, Kashiwa, Chiba 277-8577, Japan

yoshinak@east.ncc.g0.jp

Phone: +81 471331111

Fax: +81471346928
Takayuki Yoshino, MD, PhD

National Cancer Center Hospital East, 6-5-1 Kashiwanoha, Kashiwa, Chiba 277-8577, Japan

tyoshino@east.ncc.g0.jp

Phone: +81 471331111

Fax: +81471346928 
treatment than were those with a mutation (tissue-positive patients: ORR, 36\% vs $0 \%$; ctDNA-positive patients, ORR, $32 \%$ vs $0 \%$ ). Decreased ctDNA fraction and HER 2 plasma copy number at 3 weeks corresponded to the therapeutic response. In the BRAVERY study, the efficacy and safety of eribulin monotherapy were investigated in patients with tissue- or ctDNA-based BRAF V600E mutations. ${ }^{38}$ Among the 27 enrolled patients, the ORR was $0 \%$, the disease control rate (DCR) was $41 \%$, the median PFS was 1.4 months, and the OS was
5.2 months. Patients with RAS/AKT and epithelial mesenchymal transition-related gene alterations in ctDNA had a worse DCR (25\% vs 54\%) and PFS (HR, 2.78; P $=.02)$. At the time of disease progression, acquired gene alterations, such as KRAS and FGFR 1 amplification, were detected.

\section{FIGURE २. GOZILA UMBRELLABBASKET TRIALS}

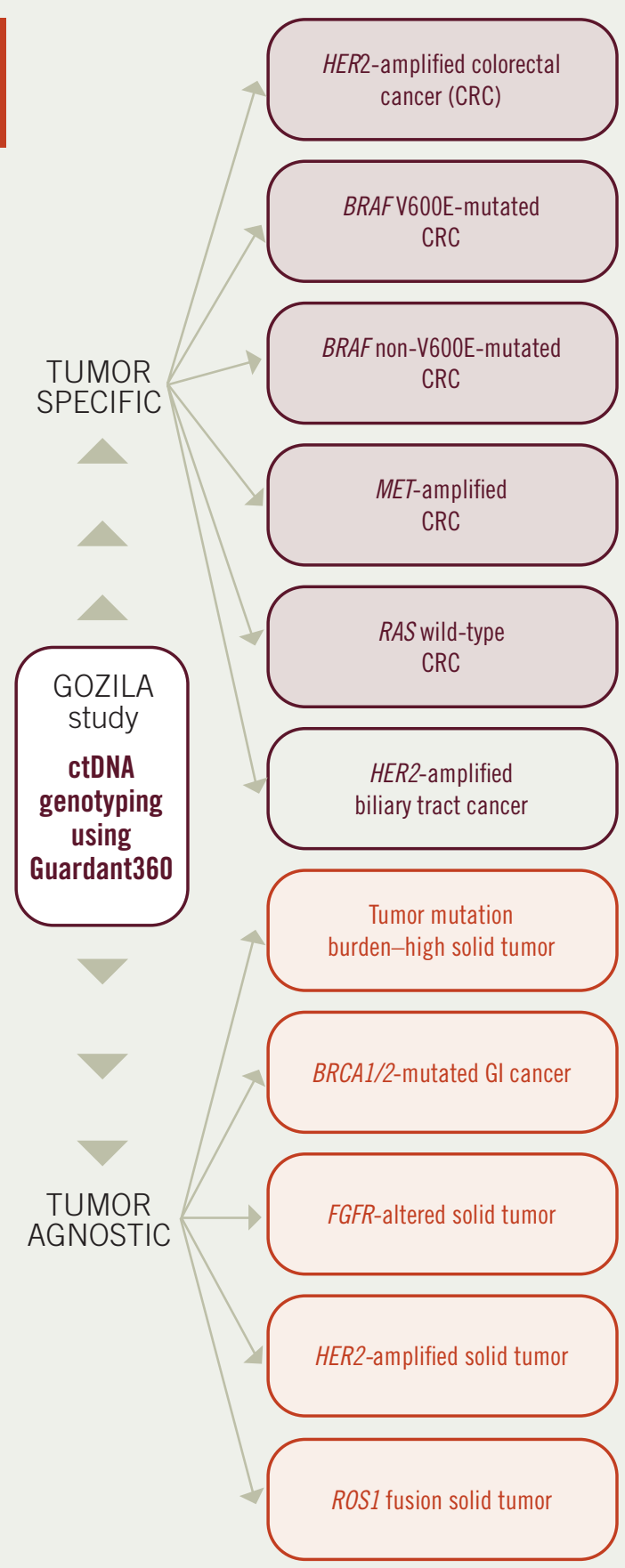

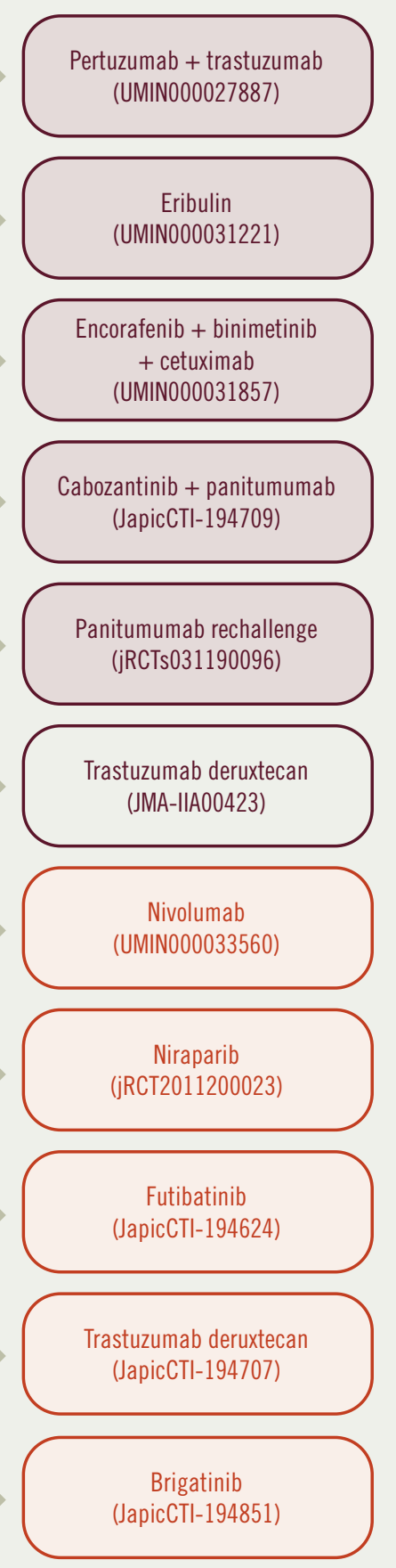




\section{TABLE २. Comparison of GOZILA and COLOMATE Studies}

\begin{tabular}{|c|c|c|}
\hline & GOZILA & COLOMATE \\
\hline Phase & $\begin{array}{l}\text { Not applicable (GOZILA is an } \\
\text { observational screening study for } \\
\text { related clinical trials) }\end{array}$ & Phase II \\
\hline Design & Basket/Umbrella & Umbrella \\
\hline Cancer type & $\begin{array}{l}\text { Solid tumors (mainly gastrointesti- } \\
\text { nal cancer) }\end{array}$ & Colorectal cancer \\
\hline Eligible patients & $\begin{array}{l}\text { Metastatic and/or unresectable } \\
\text { cancer at any line of chemotherapy }\end{array}$ & $\begin{array}{l}\text { Metastatic and/or } \\
\text { unresectable can- } \\
\text { cer after standard } \\
\text { chemotherapy }\end{array}$ \\
\hline Target number of patients & 5000 & 500 \\
\hline Study sites & 31 & 16 \\
\hline \multicolumn{3}{|l|}{ Related target therapies } \\
\hline HER2 & Yes & Yes \\
\hline BRAF V600E & Yes & Yes \\
\hline$B R A F$ non-V600E & Yes & Yes \\
\hline MET amplification & Yes & Yes \\
\hline $\begin{array}{l}\text { Anti-EGFR antibody } \\
\text { rechallenge }\end{array}$ & Yes & Yes \\
\hline FGFR alterations & Yes (tissue-agnostic approach) & Yes \\
\hline ROS1 fusion & Yes (tissue-agnostic approach) & No \\
\hline BRCA mutation & Yes (tissue-agnostic approach) & No \\
\hline
\end{tabular}

GOZILA provides the broad screening platform for various umbrella/basket clinical trials for targeted therapies, including those in which the targets are ERBB2 (HER2), BRAF V600E, BRAF non-V600E, and MET amplifications; FGFR alterations; ROS1 fusions; BRCA mutations; and TMB. The TRIUMPH study results suggest promising ORRs, PFS, and OS in patients with ctDNA RAS wild-type and HER2-amplified mCRC. Wild-type RAS, BRAF, PIK3CA, and $H E R 2$ were also predictors of response to study treatment.

\section{COLOMATE}

COLOMATE is a phase 2 umbrella screening trial sponsored by the Academic and Community Cancer Research United consortium. Actionable genomic alterations in patients with $\mathrm{mCRC}$ are investigated using Guardant $360^{\mathrm{TM}}$, and the impact of molecularly assigned therapy is assessed
(MOUNTAINEER; NCT03043313); tepotinib (Tepmetko) plus cetuximab for patients with MET amplification; anti-BRAF treatments for patients with $B R A F$ non-V600E mutation or as rechallenge; and pemigatinib for patients with FGFR alterations (Table 2). Additional companion studies are currently underway.

The primary objectives of COLOMATE are to perform blood-based genomic profiling of patients with treatment-refractory mCRC to facilitate accrual to molecularly assigned therapies and to obtain patient-matched tumor tissue and ctDNA from peripheral blood to facilitate clinically annotated genomic analyses. Secondary correlative objectives are to explore the mechanisms of acquired resistance to molecularly assigned therapy and to explore the correlation between ctDNA-based mutational burden (eg, allele frequency, copy number) and clinical outcomes, such as objective response rate, ORR, PFS, and OS.

MOUNTAINEER is an open-label, pivotal phase 2 trial that initially consisted of a single cohort of up to 45 patients (cohort A) treated with tucatinib plus trastuzumab. Interim analysis of the initial 26 patients enrolled in MOUNTAINEER demonstrated an ORR of $52.2 \%$ (12 partial responses in 23 evaluable patients), median duration of response of 10.4 months, median PFS of 8.1 months, and median OS of 18.7 months. ${ }^{39}$ The trial was expanded to include an additional 70 patients randomized 4:3 into 2 cohorts: Cohort B $(\mathrm{N}=40)$, who will receive tucatinib plus trastuzumab, and Cohort $\mathrm{C}(\mathrm{N}=30)$, who will receive tucatinib monotherapy.

\section{Differences and similarities between GOZILA and COLOMATE}

In comparing the study designs of GOZILA and COLOMATE, we observed several differences and similarities ( $\mathrm{Ta}$ ble 2). Only patients with metastatic and/or unresectable CRC are eligible for 
COLOMATE, whereas patients with all types of metastatic and/or unresectable GI cancers, breast cancers, and other types of solid tumors with specific gene alterations are eligible for GOZILA. Furthermore, patients at any line of treatment are eligible for GOZILA; as such, its target population is 5000. In contrast, effective screening of candidate patients for companion trials is key for COLOMATE. Using the rapid turnaround time from consent to treatment recommendation by ctDNA-based NGS assay, at least $35 \%$ of patients will qualify for a companion trial.

When focusing just on patients with $\mathrm{mCRC}$, the companion trials with targeted therapies are similar between GOZILA and COLOMATE (Table 2). Both groups have identified patients for studies examining HER2 amplification, BRAF V600E mutations, BRAF non-V600E mutations, MET amplification, FGFR alterations, and anti-EGFR antibody rechallenge. Because patients with various types of solid tumors can be eligible for GOZILA, some other basket studies for TMB, FGFR alterations, HER 2 amplification, ROS1 fusion, and $B R C A$ mutation are accompanied by tissue-agnostic approaches.

\section{Discussion and conclusions}

ctDNA-based genotyping has emerged as a potential tool for evaluating primary and acquired gene alterations in real time. Because the use of ctDNA-based NGS assays is feasible for detecting ctDNA in most patients with GI cancer, ${ }^{12}$ ctDNA-based approaches can be the future standard platform for basket/umbrella target-based trials. Indeed, companion trials for HER2, $B R A F$ V600E, BRAF non-V600E, and FGFR alterations, as well as MET amplification and anti-EGFR antibody rechallenge, are ongoing in both GOZILA and COLOMATE.

According to the data of ctDNA-based GOZILA and tissue-based GI-SCREEN, ctDNA-based NGS significantly improved sample unavailability $(0.3 \%$ vs $1.5 \%)$ and failure rate $(0.1 \%$ vs $10.6 \%)$.

12 On the other hand, several factors related to lower detection in ctDNA-based analyses have been reported. In GI cancers, lower detection rates of ctDNA were suggested in patients with PDAC and GEA, and in patients with lung-only or peritoneum-only metastatic sites in mCRC. ${ }^{12,40}$ Lower detections of ctDNA were also reported in patients with early or localized disease ${ }^{41}$ and in patients undergoing intensive chemotherapy. ${ }^{42}$ These factors must be taken into consideration when we apply the ctDNA-based genomic analyses.

Although, compared with tissue-based GI-SCREEN, ctDNA-based GOZILA significantly increased the relative proportion of patients enrolled in clinical trials for targeted therapies with similar efficacies, ${ }^{12}$ only $9.5 \%$ of screened patients were enrolled. To detect further targeted gene alterations and increase enrollment in related clinical trials, we are currently preparing several additional tissueagnostic studies. Furthermore, wholeexome sequencing (WES) and RNA-based NGS may uncover known fusion events in unexpected tumor histologies and identify novel fusion events with potential clinical relevance. ${ }^{43-45}$ We are now preparing next-generation comprehensivescreening platforms to investigate tissue-based WES and circulating tumor nucleic acid detection, including both ctDNA and ctRNA. Additionally, multi-immunofluorescent staining will be performed to screen a patient's immune status, including the presence of tumor-infiltrating lymphocytes, macrophages, and immune checkpoint proteins (MONSTAR-SCREEN-2).

In conclusion, both GOZILA and COLOMATE are well-qualified ctDNAbased screening platforms for companion targeted therapies, especially in patients with mCRC. The existence of various companion trials for the same target alteration can lead to future international collaboration with the use of these 2 screening platforms.

\section{*AUTHORS HAVE equal Contribution}

FINANCIAL DISCLOSURE: HB reports research funding from AstraZeneca, Sysmex, and honoraria from Taiho Pharmaceutical and Eli Lilly Japan; YN reports research grants from Taiho Pharmaceutical, Chugai Pharmaceutical, Guardant Health, Genomedia, Daiichi-Sankyo, and Seagen;

DK reports honoraria from Taiho, Ono, DaiichiSankyo, Pfizer, Takeda, Lilly, Merck Biopharma, MSD, Bristol-Myers Squibb, Chugai, and Sysmex; research funding from Ono, MSD, Novartis, IQVIA, Syneos Health, and CMIC ShiftZero; TY reports research grants from Taiho Pharmaceutical, Sumitomo Dainippon, Ono Pharmaceutical, Chugai Pharmaceutical, Amgen, Parexel International, MSD, Daiichi Sankyo, Sanofi.

$\mathbf{1}$ For full reference list, visit cancernetwork.com/Bando_7.21

\section{Bando}

is head physician in the Department of Gastroenterology and Gastrointestinal Oncology, National Cancer Center Hospital East.

\section{Kotani}

is a staff physician in the Department of Gastroenterology and Gastrointestinal Oncology, National Cancer Center Hospital East.

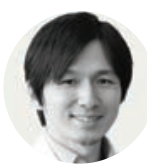

Nakamura

is a staff physician in the Department of Gastroenterology and Gastrointestinal Oncology, National Cancer Center Hospital East in Kashiwa, Japan.

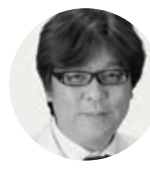

Yoshino

is director of the Department of Gastroenterology and Gastrointestinal Oncology, National Cancer Center Hospital East. 


\section{Level of Scientific Evidence Underlying the National Comprehensive Cancer Network Clinical Practice Guidelines for Hematologic Malignancies: Are We Moving Forward?}

Madhuri Chengappa*, MBBS; Aakash P. Desai*, MBBS, MPH; Ronald S. Go, MD; and Thejaswi K. Poonacha, MD, MBA

\section{ABSTRACT}

BACKGROUND: The level of scientific evidence in National Comprehensive Cancer Network (NCCN) guidelines for malignant hematological conditions haven't been recently investigated. We describe the distribution of categories of evidence and consensus (EC) among the 10 most common hematologic malignancies with regard to recommendations for staging, initial and salvage therapy, and surveillance.

METHODS: We reviewed the level of evidence for the 10 most common hematological malignancies by incidence in the United States as of 2020. The NCCN definitions for EC are: category 1, high level of evidence, such as randomized controlled trials, with uniform consensus; category $2 \mathrm{~A}$, lower level of evidence with uniform consensus; category 2B, lower level of evidence without a uniform consensus but with no major disagreement; and category 3, any level of evidence but with major disagreement. We compared our results with previously published results from 2011.
RESULTS: Of 1353 recommendations, 5\%, $91 \%, 4 \%$, and $1 \%$ fell into EC categories 1, 2A, 2B, and 3 , respectively, while in 2011 the comparable percentages were $3 \%, 93 \%, 4 \%$, and $0 \%$, respectively. Recommendations with category 1 EC were found in all guidelines, except for Burkitt lymphoma. Of all therapeutic recommendations, $6.3 \%$ were category 1 $\mathrm{EC}$, with the majority of these (56.4\%) pertaining to initial therapy. Guidelines with highest proportions of therapeutic recommendations with category $1 \mathrm{EC}$ were multiple myeloma (12.4\%), chronic lymphocytic leukemia/small lymphocytic lymphoma (6.9\%), and acute myeloid leukemia (5.6\%).

CONCLUSIONS: Recommendations in the 2020 NCCN guidelines are largely developed from lower levels of evidence but with uniform expert opinion, underscoring the urgent need and available opportunities to expand the current evidence base in malignant hematological disorders. 


\section{Introduction}

Evidence-based medicine (EBM) is an interdisciplinary approach that integrates clinical experience and patient values with the best available research information to make clinical decisions for individual patients. Health care providers always seek to base their decisions on the best available evidence. EBM aims for the ideal that health care professionals should make "conscientious, explicit, and judicious use of current best evidence" in their everyday practice. ${ }^{1}$

EBM has improved the practice of medicine by developing methods and techniques for generating systemic reviews and clinical practice guidelines (CPGs). ${ }^{2}$ The Institute of Medicine defines CPG as "statements that include recommendations, intended to optimize patient care, that are informed by a systematic review of evidence and an assessment of the benefits and harms of alternative care options." ${ }^{3,4}$ CPGs are an essential tool to translate evidence-based medicine into practice and have an emerging role in national policy to guide reimbursement and to serve as the standard of care in medical malpractice cases. ${ }^{3}$ Within hematology, several organizations provide CPGs for medical practitioners as practice guidance, including the American Society of Hematology (ASH), European Hematology Association, and National Comprehensive Cancer Network (NCCN). In the United States, NCCN guidelines are the most comprehensive standard for clinical care in malignant hematology, and they are widely used by clinicians and payers.

The NCCN is a not-for-profit alliance of 31 leading US cancer centers. designated as National Cancer Institutes (NCI). The organization has engaged in the development of clinical practice guidelines for the past years, utilizing the best contemporary evidence and expert consensus. ${ }^{5}$ Currently, 73 NCCN guidelines for different forms of cancer cover cancer detection, prevention and risk reduction, work-up and diagnosis, treatment, and supportive care. These guidelines

\section{FIGURE 1. Comparison Between the Number of Recommendations per}

\section{Guideline in 2011 and 2020}

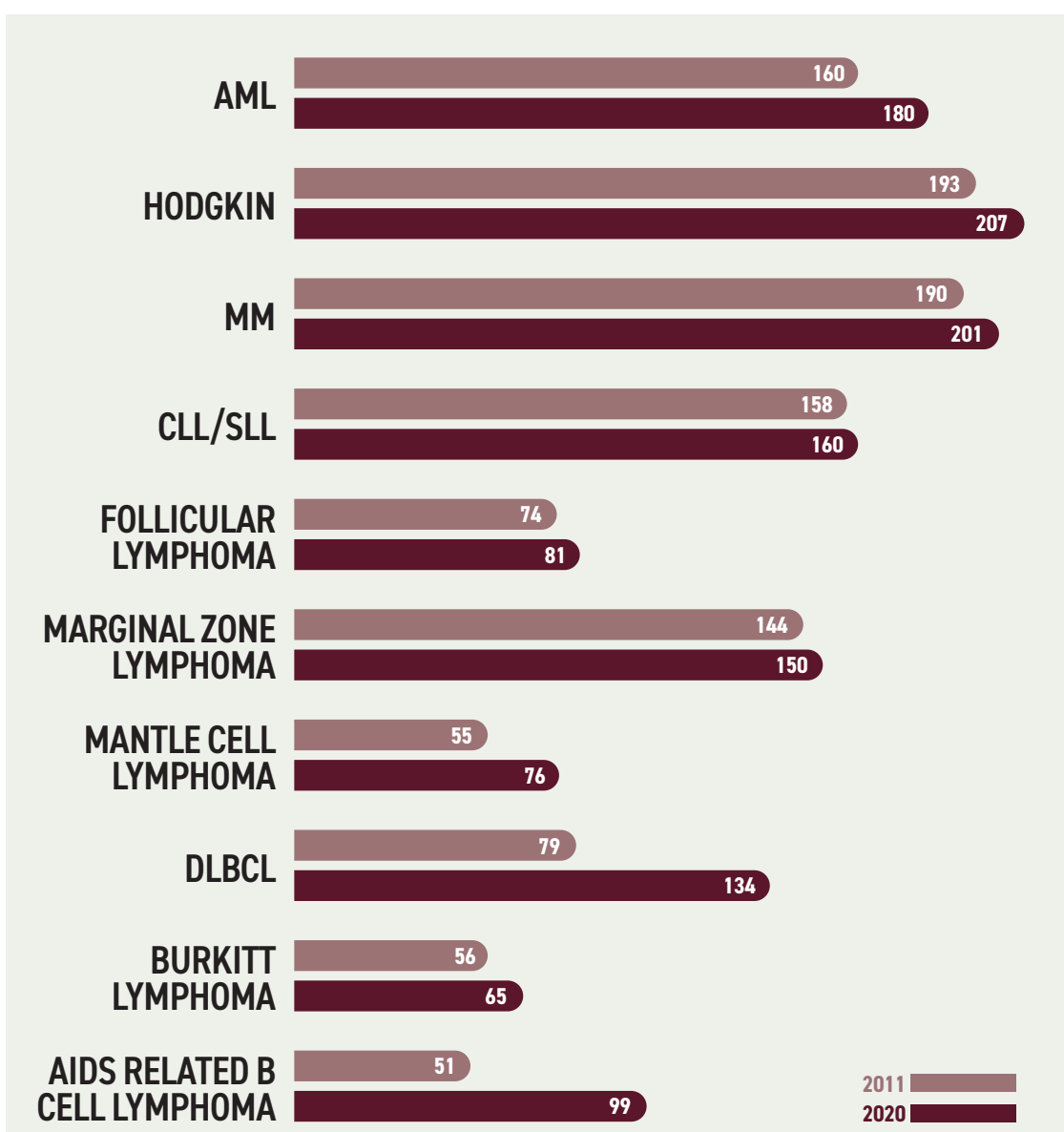

AML, acute myeloid leukemia; MM, multiple myeloma; CLL, chronic lymphocytic leukemia; SLL, small lymphocytic leukemia; DLBCL, diffuse large B-cell lymphoma.

have become important documents for guiding clinical practice, insurance reimbursements, and quality improvement initiatives around the globe. ${ }^{6}$ The guidelines are developed and updated by 60 individual panels comprised of more than 1520 members who are both clinicians and researchers. These guidelines are updated at least annually. ${ }^{7}$

The authors of a review of the 2011 NCCN clinical practice guidelines found that only $3 \%$ of the recommendations were based on high levels of evidence. ${ }^{8}$ Given that the vast majority of guidelines in malignant hematology were based on lower levels of evidence, a need was not- ed for increased comparative effectiveness research and compliance studies within the field of hematology. However, the categories of evidence and consensus in NCCN guidelines for malignant hematological conditions have not been recently investigated. In this study, we aimed to describe the distribution of categories of evidence and consensus (EC) among the 10 most common hematologic malignancies with regard to recommendations for staging, initial and salvage therapy, and surveillance. We also sought to evaluate the changes in level of evidence between the most recent NCCN guidelines, from 2020, and those published in 2011. 


\section{FIGURE 2. Distribution of Catego- ries of Evidence and Consensus for Guidelines in 2011 (A) and 2020 (B)}
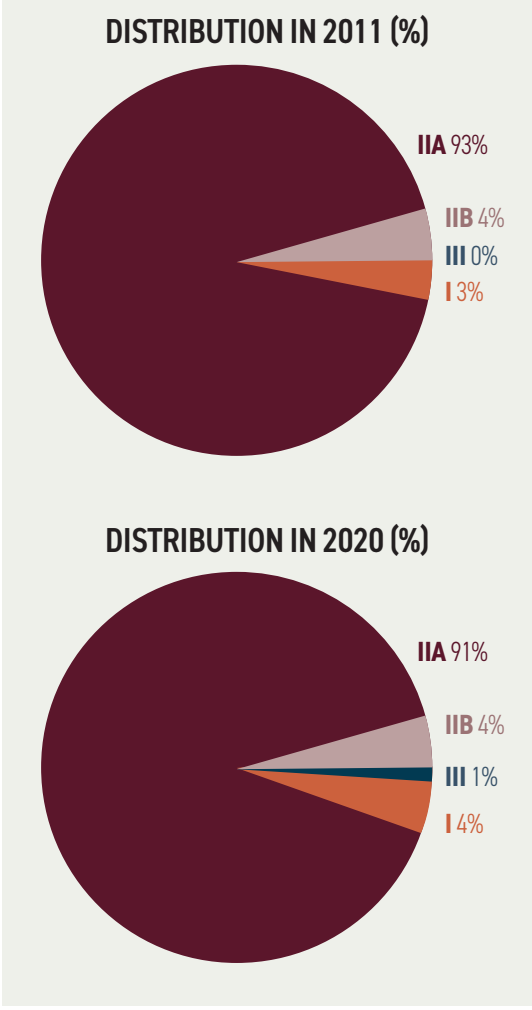

\section{Methods}

From the NCCN website, we obtained the latest versions (as of July 2020) of CPGs for relevant hematological malignancies?: acute myeloid leukemia (AML), multiple myeloma (MM), Hodgkin lymphoma (both adult and adolescent/young adult), diffuse large B-cell lymphoma (DLBCL), follicular lymphoma, chronic lymphocytic leukemia/small lymphocytic lymphoma (CLL/SLL), mantle cell lymphoma (MCL), marginal zone lymphoma (MZL), AIDSrelated B-cell lymphoma, and Burkitt lymphoma. Only comprehensive guidelines were included in this study; NCCN evidence blocks, quick guide, NCCN framework and guidelines for patients were not included. ${ }^{9-14}$

We reviewed the number of recommendations included in the CPGs for each malignancy and the level of evidence associated with each recommendation. ${ }^{15,16}$
We abstracted the recommendations after carefully reviewing the content within each guideline document and the displayed text within the graphical algorithms. We enumerated the EC categories for each of the guidelines according to the following 4 major areas of recommendations: staging, initial treatment, salvage treatment, and surveillance.

The NCCN definitions for various EC categories are as follows: (A) category 1: high-level evidence with uniform NCCN consensus that the intervention is appropriate; (B) category 2A: lower-level evidence with uniform NCCN consensus that the intervention is appropriate; (C) category 2B: lower-level evidence without a uniform NCCN consensus that the intervention is appropriate but with no major disagreement; and (D) category 3: any level of evidence but with major disagreement that the intervention is appropriate. ${ }^{9}$ Data obtained from the current guidelines as of July 2020 were compared with previously published results from November 2011. ${ }^{8}$

\section{Results}

The ten July 2020 guidelines included a total of 1353 recommendations. Of the 1353 recommendations, $5 \%, 91 \%, 4 \%$, and $1 \%$ fell into categories $1,2 \mathrm{~A}, 2 \mathrm{~B}$, and $3 \mathrm{EC}$, respectively; the comparable percentagesinNovember 2011 were $3 \%, 93 \%, 4 \%$, and $0 \%$, respectively. Recommendations with category $1 \mathrm{EC}$ were for $\mathrm{MM}$ (12\%), CLL/SLL (7\%), AML (5\%), follicular lymphoma (4\%), DLBCL (4\%), MCL (3\%), Hodgkin lymphoma (2\%), AIDS-related B-cell lymphoma (2\%), and MZL $(1 \%)$. No category 1 recommendations were noted in Burkitt lymphoma. Of all 980 therapeutic recommendations, $6.3 \%$ were in category $1 \mathrm{EC}$; the majority of these, $56.4 \%$, pertained to initial therapy. MM (12.4\%), CLL/SLL (6.9\%), and AML $(5.6 \%)$ guidelines had the highest proportions of category 1 therapeutic recommendations. No category 1 recommendations were made for staging or surveillance guidelines.
In comparison with the guidelines studied in 2011, the total number of recommendations in 2020 increased by $16.6 \%$, rising from 1160 to 1353 (Figure 1). Hodgkin lymphoma continued to have the most recommendations $(\mathrm{n}=207)$. AIDS-related B-cell lymphoma had the least recommendations in 2011 ( $\mathrm{n}$ $=51$ ), whereas in 2020, Burkitt lymphoma had the least $(n=65)$. The majority of recommendations both in 2011 and 2020 were based on category 2A EC. While a small fraction of recommendations were based on category $1 \mathrm{EC}$, that percentage did improve from $3 \%$ to $4 \%$ (Figure 2). Majority of the recommendations in this $1 \%$ increase were in the therapeutic category.

Figure 4 shows the similarity of the dis-

\section{FIGURE 3. Distribution of Areas of Recommendations in 2011 (A) and 2020 (B)}
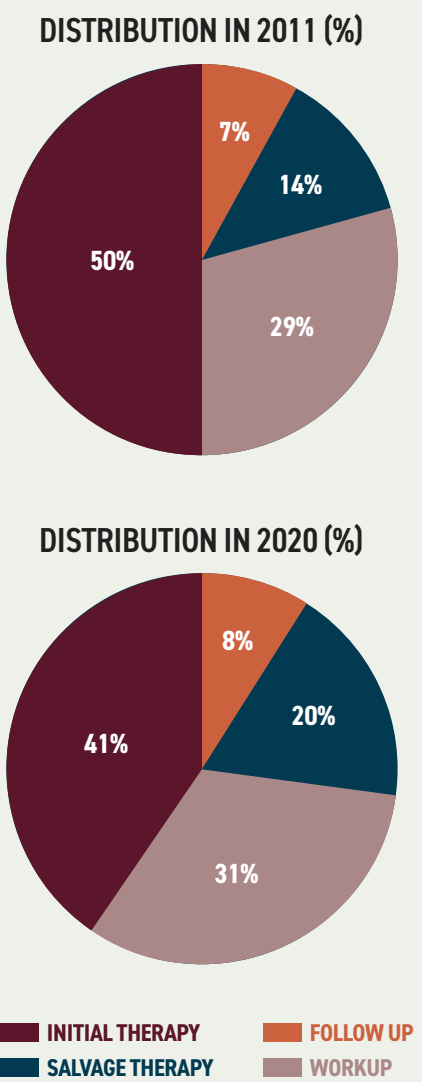


\section{FIGURE 4. Distribution of Evidence and Consensus Categories According to Area of Recommendation for All Guidelines in 2011 and 2020}

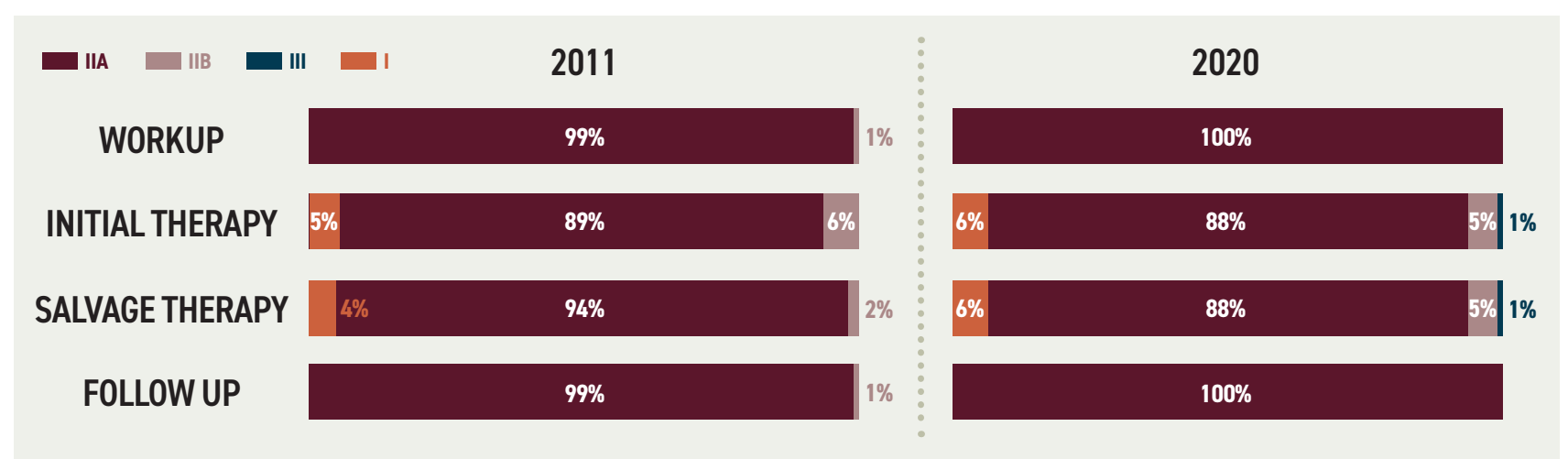

tribution of recommendations among the major areas in both years studied. The distribution of evidence among the different types of recommendations demonstrated that $5 \%$ of initial therapy recommendations and $4 \%$ of salvage therapy recommendations had category 1 EC in 2011; those figures both increased to $6 \%$ in 2020, suggesting improved evidence in the therapeutic recommendations section of the guidelines (Figure 4). Figure 5 illustrates the distribution of categories of evidence and consensus for each CPG. Nearly all recommendations on staging and surveillance were based on category 2A EC, with none having category $1 \mathrm{EC}$, both in 2011 and 2020 .

In 2011, the highest proportions of category 1 recommendations were found in the follicular lymphoma guidelines $(11 \%)$. No category 1 recommendations were found for CLL/SLL, MZL, MCL, AIDS-related B-cell lymphoma, or Burkitt lymphoma. In 2020, MM (12\%) had the highest proportion of category 1 recommendations. Between 2011 and 2020(Figure 6), the proportion of category 1 recommendations increased in MM, CLL/SLL, MCL, MZL, and AIDS-related B-cell lymphoma; CLL/ SLL had a significant increase in category 1 recommendations from $0 \%$ (2011) to $7 \%(2020)$. Burkitt lymphoma did not have any category 2 recommendations. Category 1 recommendations for follicular lymphoma had a downward trend from $11 \%$ (2011) to $4 \%$ (2020).

\section{Discussion}

Our study describes the distribution of EC categories among the 10 most common hematologic malignancies with regard to recommendations for staging, initial and salvage therapy, and surveillance based on the latest data from 2020 in comparison with previously published data from 2011. Overall, we found that the total number of recommendations increased from 2011 to 2020 by $16.6 \%$, with less than $10 \%$ of recommendations based on high-level evidence, characterized as category 1 . The proportion of category 1 recommendations increased in all CPGs except follicular cell lymphoma and Burkitt's lymphoma. In fact, follicular cell lymphoma had a decrease in category 1 recommendations. No category $1 \mathrm{EC}$ recommendations were found in screening or surveillance. Despite the increase in the total number of recommendations, the distribution of different categories of EC recommendations were largely similar to those of 2011.

Over the past 25 years, the NCCN has evolved its CPGs, and it currently has 73 CPGs that are developed and updated by 60 individual panels, composed of more than 1520 clinicians and oncology researchers from 31 NCCN member institutions. ${ }^{17} \mathrm{NCCN}$ releases CPGs, developed as algorithms or flowcharts, that cover more than $97 \%$ of all cancers affecting patients in United States and are updated several times per year. The recommendations and updates are based on the best and latest available scientific evidence integrated with expert judgment of leading clinicians, and they are intended to assist all individuals who impact decision-making in cancer care, including physicians, nurses, pharmacists, payers, patients, and families. NCCN CPGs are the most widely used CPGs for cancer care in the world and have been translated into more than 70 languages.

The NCCN guideline development panel comprises the NCCN guidelines steering committee, panels specific to each of the guidelines, and the NCCN headquarters team, which supports the panels and the activities of guideline development. Each NCCN guidelines panel consists of a panel chair, vice-chair (or cochair), and a group of representatives from the NCCN member institutions. The NCCN guideline development process consists of annual institutional review of the guidelines by the experts at the NCCN member institutions. They follow an elaborate protocol, ultimately resulting in voting if the discussion calls for a change in guidelines. The panel chair and members then develop the specific recommendations as algorithms. The 
scientific rationale and clinical evidence supporting the recommendations outlined in the algorithms are provided in the discussion sections. ${ }^{9}$

Per the NCCN, "the level of evidence depends upon the following factors, which are considered during the deliberation process by the panel: quality of data (eg, trial design and how the results/ observations were derived [randomized controlled trials (RCTs), non-RCTs, meta-analyses or systematic reviews, clinical case reports, case series]), quantity of data (eg, number of trials, size of trials, clinical observations only), and consistency of data (eg, similar or conflicting results across available studies or observations)." The determination of level of evidence is subjective and is based on the panel's decision, depending on the quality, quantity, and consistency of the available data. The degree of consensus among the panelists is based on the percentage of panel votes.

Per NCCN's policy on development and update of guidelines, "for uniform NCCN consensus defined in category 1 and category 2A, a majority panel vote of at least $85 \%$ is required. For the "NCCN consensus" defined in category $2 \mathrm{~B}$, a panel vote of at least $50 \%$ (but less than $85 \%$ ) is required. Lastly, for recommendations where there is strong panel disagreement regardless of the quality of the evidence, NCCN requires a panel vote of at least $25 \%$ to include and designate a recommendation as category 3.” The NCCN guideline document specifies that when categories are not explicitly mentioned within the guide- lines, the default designation for such recommendation is category $2 \mathrm{~A}$.

\section{Some of the limitations of NCCN guideline development process are that:}

- the literature search strategy, inclusion and exclusion criteria, and data extraction are not described in detail;

- in the current NCCN definition of categories of EC, it seems that the level of evidence is equated with the quality of evidence. High-level evidence (from RCTs) may be of low quality if serious study limitations and biases are present. Conversely, lower-level evidence (observational studies or phase 2 trials) may be of high quality if they show consistent and a large magnitude of treatment effect; and

\section{FIGURE 5. Distribution of Categories of Evidence and Consensus According to Type of Cancer Guidelines in 2011} and 2020
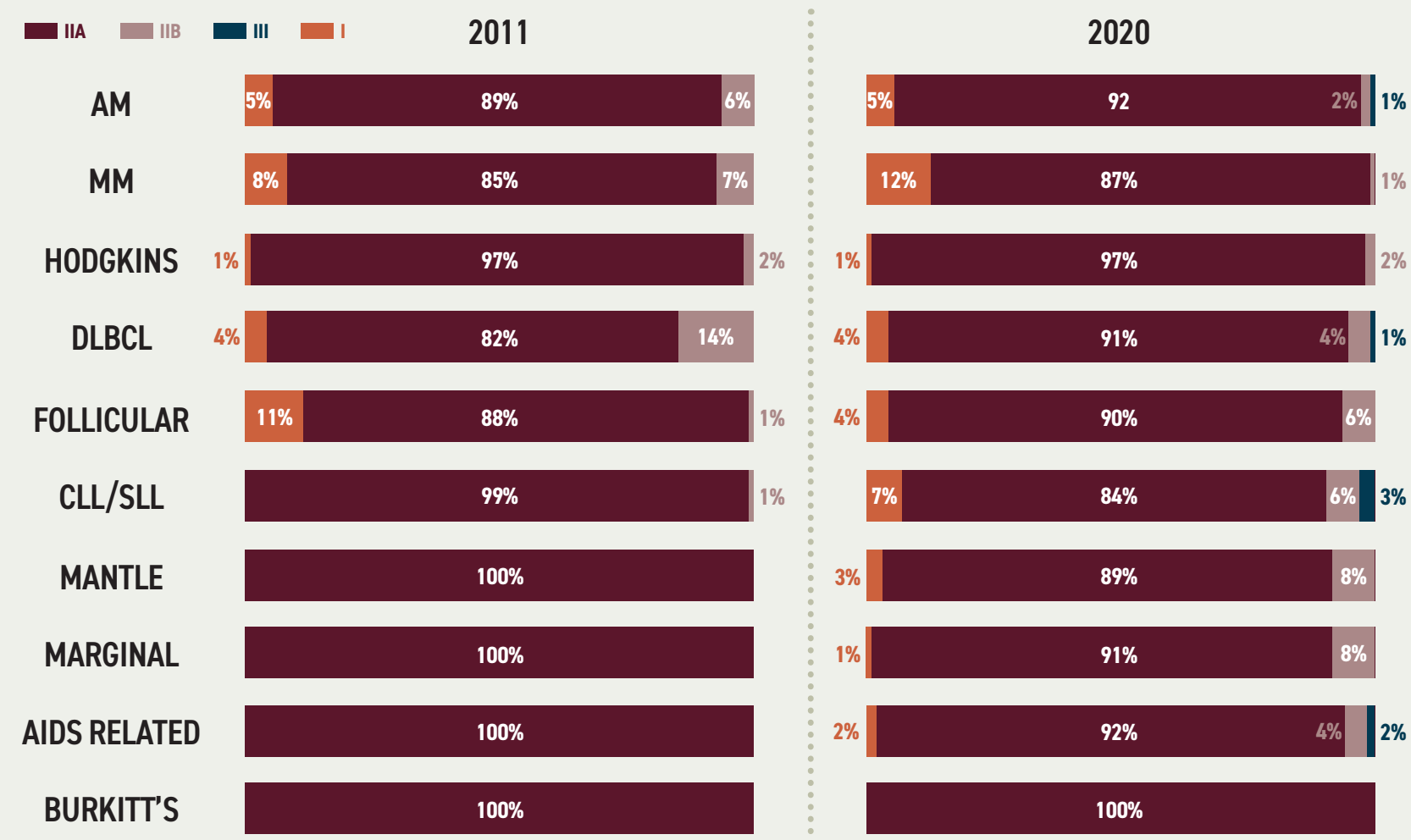

AML, acute myeloid leukemia; CLL, chronic lymphocytic leukemia; DLBCL, diffuse large B-cell lymphoma; MM, multiple myeloma;

MCL, mantle cell lymphoma; MZL, marginal zone lymphoma; SLL, small lymphocytic leukemia. 
- the panel decisions on the quality of evidence are not separated from the strength of recommendation. High-quality evidence may be worthy of only a weak recommendation if uncertainty or variability exists in terms of risks and benefits or personal values and preferences. On the contrary, strong recommendations may arise from lower-quality evidence.

Over the past 2 decades the Grading of Recommendations Assessment, Development and Evaluation (GRADE) working group has developed a common, sensible, and transparent approach to grading quality (or certainty) of evidence and strength of recommendations. ${ }^{17}$ It quantifies the strength of recommendations taking into account the estimate of effect of an intervention; the design, execution, consistency, precision, and reporting bias of studies; the patient health benefits vs harms; and the health system costs. ${ }^{18}$ Many international organizations provide input into the development of the GRADE approach, which is now considered the standard in guideline development. Interestingly, NCCN and other major cancer organizations such as the American Society of Clinical Oncology and Cancer Care Ontario have not adopted GRADE. However, some of the guidelines developed by ASH were based on GRADE. More recently, the European Society of Medical Oncology has adopted the GRADE system as described in their clinical practice guidelines standard operating procedures. If the GRADE system were used by NCCN, the distribution of EC for each guideline would potentially be different.

The relative lack of high-level evidence is not unique to the recommendations arising from NCCN's hematological malignancies' CPG. A similar comparative study of NCCN CPGs for the 10 most common cancers in the United States demonstrated that although the number of recommendations increased between 2010 and 2019, the relative distribution of category of evidence and consensus

\section{FIGURE 6. Trends in Proportion of Category 1 Recommendations From 2011} to 2020

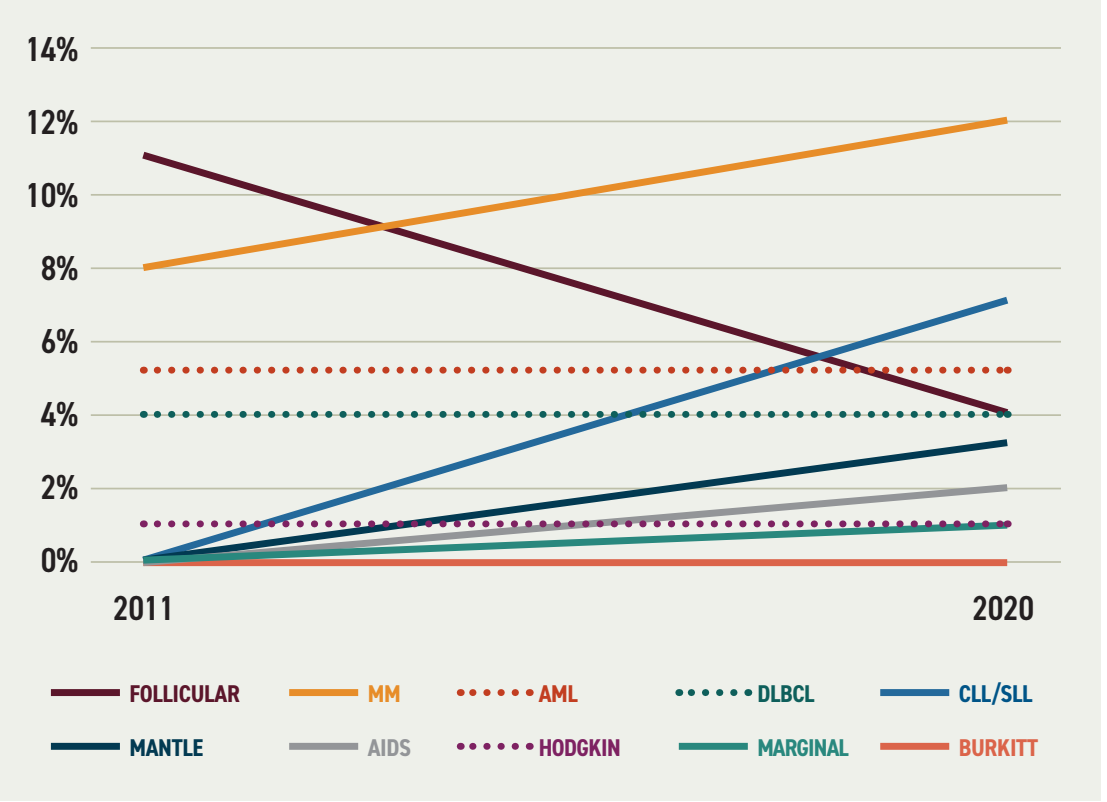

AML, acute myeloid leukemia; CLL, chronic lymphocytic leukemia; $D L B C L$, diffuse large B-cell lymphoma; MM, multiple myeloma; MCL, mantle cell lymphoma; MZL, marginal zone lymphoma; SLL, small lymphocytic leukemia.

remained essentially the same. ${ }^{16}$ Similar studies in cardiology have shown that American College of Cardiology/American Heart Association guidelines did not demonstrate any major changes in the level of evidence among CPGs between 2010 and 2019. ${ }^{19}$ In our study, we found that $5 \%$ of the recommendations were based on high levels of evidence, representing a $2 \%$ increase in comparison with 2011. It is reassuring that most of the increase in level of evidence was found in recommendations for treatment.

The National Cancer Institute has been proactive in assimilating communitybased clinical practices to pursue research in oncology and serve as sites for research trials to improve and boost the evidence base. However, these sites face challenges, partly as a result of emerging science, technology, genomics, and molecular target therapy, but more significantly because of changing health care policies and federal support for research. The entry of the pharmaceutical industry into cancer research and their collaboration with academic institutions have also increased the evidence base for cancer diagnoses and treatment. However, in some cases, financial conflicts of interest (FCOI) and the potentially disparate objectives of pharmaceutical companies and academic groups may limit the companies' overall contribution. ${ }^{20}$ While no study results directly correlate issues of FCOI and their impact on guidelines, a recent study concluded that of all the NCCN guidelines, $46 \%$ had of guideline panelists reporting at least 1 episode of FCOI. ${ }^{21}$ Relatedly, another recent study on trends in industry payments to physicians between 2014 and 2018 showed that physicians receiving total payments of more than $\$ 50,000$ from the pharmaceutical industry continued to receive similar or greater amounts, perhaps reflecting evolving industry strategy that concentrates payments, for which greater return on investment is anticipated. ${ }^{22}$ 
NCCN acknowledges that the quality of existing evidence in cancer research is highly variable. Large, well-designed RCTs may provide high-quality clinical evidence in some tumors and clinical situations. However, much of the clinical evidence available to clinicians is primarily based on data from indirect comparisons among randomized trials, phase 2 or nonrandomized trials, or, in many cases, on limited data from multiple smaller trials, retrospective studies, or clinical observations. ${ }^{23}$ Notably, only approximately $16 \%$ of NCCN recommendations are based on the results of randomized phase 3 trials. ${ }^{24}$

In situations where high-level evidence does not exist, guidelines are derived from critical evaluation of evidence, integrated with the clinical expertise and consensus of a multidisciplinary panel of cancer specialists, clinical experts, and investigators. The guidelines are updated regularly to ensure their transparency, and a "transparency document" is posted on the NCCN website, detailing changes made to recommendation category or indication of drugs or biologics, a short summary of the panel discussion, and the rationale for the change. In addition, all external submission requests are available for viewing on the NCCN website along with the transparency document. ${ }^{7}$ One could argue about the subjective nature of recommendations, as multiple options exist for 1 specific presentation.
This is because many effective regimens were not compared head-to-head in phase 3 trials. However, this allows individualization of therapy to patients based on comorbidities and other variables. Therefore, the subjective nature may be to the advantage of clinicians when high levels of evidence do not exist.

\section{Creating awareness [about current research needs] and prioritization of resources may help improve the level and quality of evidence informing the care that we offer to our patients.}

While creating high-level evidence is not the primary responsibility of NCCN, one way to draw attention to required areas of research is by identifying recommendations that are largely based on lower levels of evidence. The current NCCN guidelines do not differentiate between low levels of evidence and no evidence, but this distinction becomes extremely important to outline areas of unmet need. Furthermore, funding could be prioritized to those areas where further research is critical.

\section{Conclusions}

Recommendations in the 2020 NCCN guidelines for hematological malignancies are mostly derived from lower levels of evidence and with expert opinion, which has not changed in over 10 years. While NCCN makes every effort to gather the best existing evidence, multiple challenges exist within health care, particularly cancer care, to generate high-quality evidence. Creating awareness, with emphasis on areas where there is a dearth of high-quality evidence, and prioritization of resources may help improve the level and quality of evidence informing the care that we offer to our patients.

\section{*AUTHORS HAVE equal contribution}

CONFLICTS OF INTEREST: RSG received travel reimbursement from the NCCN to chair the histiocytic neoplasm clinical practice guidelines panel. The other authors have no disclosures.

\section{AUTHOR CONTRIBUTIONS:}

MADHURI CHENGAPPA: Data curation, writing - original draft, formal analysis

AAKASH P. DESAI: Conceptualization, data curation, investigation methodology, formal analysis, review, and editing

RONALD S. GO: Conceptualization, supervision, review, editing, and validation

THEJASWI K. POONACHA: Conceptualization, supervision, review, editing, and validation

For full reference list, visit cancernetwork.com/Chengappa_7.21

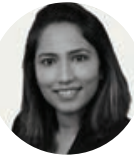

\section{Chengappa}

is from the Department of Medicine at Nazareth Hospital in Philadelphia, PA.

\section{Go}

is from the Department of Hematology at the Mayo Clinic in Rochester, MN.

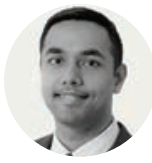

Desai

is from the Department of Hematology and Oncology at the Mayo Clinic in Rochester, MN.

\section{Poonacha}

is from the Department of Internal Medicine at the University of Minnesota Medical Center in Minneapolis, MN. 



\section{BRIEF SUMMARY OF FULL PRESCRIBING INFORMATION}

This brief summary does not include all the information needed to use GAVRETO safely and effectively.

See full Prescribing Information for GAVRETO. | GAVRETO'T (pralsetinib) capsules, for oral use. Initial U.S. approval: 2020

\section{INDICATIONS AND USAGE}

\section{Metastatic RET Fusion-Positive Non-Small Cell} Lung Cancer

GAVRETO is indicated for the treatment of adult patients with metastatic RET fusion-positive non-small cell lung cancer (NSCLC) as detected by an FDA approved test.

This indication is approved under accelerated approval based on overall response rate and duration of response. Continued approval for this indication may be contingent upon verification and description of clinical benefit in a confirmatory trial(s).

\section{RET-Mutant Medullary Thyroid Cancer}

GAVRETO is indicated for the treatment of adult and pediatric patients 12 years of age and older with advanced or metastatic $R E T$-mutant medullary thyroid cancer (MTC) who require systemic therapy.

This indication is approved under accelerated approval based on overall response rate and duration of response. Continued approval for this indication may be contingent upon verification and description of clinical benefit in a confirmatory trial(s).

\section{RET Fusion-Positive Thyroid Cancer}

GAVRETO is indicated for the treatment of adult and pediatric patients 12 years of age and older with RET fusion-positive advanced or metastatic thyroid cancer who require systemic therapy and who are radioactive iodine-refractory (if radioactive iodine is appropriate).

This indication is approved under accelerated approval based on overall response rate and duration of response. Continued approval for this indication may be contingent upon verification and description of clinical benefit in a confirmatory trial(s).

\section{WARNINGS AND PRECAUTIONS}

\section{Interstitial Lung Disease/Pneumonitis}

Severe, life-threatening, and fatal interstitial lung disease (ILD)/ pneumonitis can occur in patients treated with GAVRETO. Pneumonitis occurred in $10 \%$ of patients who received GAVRETO, including $2.7 \%$ with Grade $3-4$, and $0.5 \%$ with fatal reactions.

Monitor for pulmonary symptoms indicative of ILD/pneumonitis. Withhold GAVRETO and promptly investigate for ILD in any patient who presents with acute or worsening of respiratory symptoms which may be indicative of ILD (e.g., dyspnea, cough, and fever). Withhold, reduce dose or permanently discontinue GAVRETO based on severity of confirmed ILD.

\section{Hypertension}

Hypertension occurred in 29\% of patients, including Grade 3 hypertension in $14 \%$ of patients. Overall, $7 \%$ had their dose interrupted and $3.2 \%$ had their dose reduced for hypertension. Treatment-emergent hypertension was most commonly managed with anti-hypertension medications.

Do not initiate GAVRETO in patients with uncontrolled hypertension. Optimize blood pressure prior to initiating GAVRETO. Monitor blood pressure after 1 week, at least monthly thereafter and as clinically indicated. Initiate or adjust anti-hypertensive therapy as appropriate. Withhold, reduce dose, or permanently discontinue GAVRETO based on the severity.

\section{Hepatotoxicity}

Serious hepatic adverse reactions occurred in $2.1 \%$ of patients treated for GAVRETO. Increased AST occurred in $69 \%$ of patients, including Grade 3 or 4 in 5\% and increased ALT occurred in $46 \%$ of patients, including Grade 3 or 4 in $6 \%$. The median time to first onset for increased AST was 15 days (range: 5 days to 1.5 years) and increased ALT was 22 days (range: 7 days to 1.7 years).

Monitor AST and ALT prior to initiating GAVRETO, every 2 weeks during the first 3 months, then monthly thereafter and as clinically indicated. Withhold, reduce dose or permanently discontinue GAVRETO based on severity.

\section{Hemorrhagic Events}

Serious, including fatal, hemorrhagic events can occur with GAVRETO. Grade $\geq 3$ hemorrhagic events occurred in $2.5 \%$ of patients treated with GAVRETO including one patient with a fatal hemorrhagic event.

Permanently discontinue GAVRETO in patients with severe or life-threatening hemorrhage.

\section{Tumor Lysis Syndrome}

Cases of tumor lysis syndrome (TLS) have been reported in patients with medullary thyroid carcinoma receiving GAVRETO. Patients may be at risk of TLS if they have rapidly growing tumors, a high tumor burden, renal dysfunction, or dehydration. Closely monitor patients at risk, consider appropriate prophylaxis including hydration, and treat as clinically indicated.

\section{Risk of Impaired Wound Healing}

Impaired wound healing can occur in patients who receive drugs that inhibit the vascular endothelial growth factor (VEGF) signaling pathway. Therefore, GAVRETO has the potential to adversely affect wound healing.

Withhold GAVRETO for at least 5 days prior to elective surgery. Do not administer for at least 2 weeks following major surgery and until adequate wound healing. The safety of resumption of GAVRETO after resolution of wound healing complications has not been established.

\section{Embryo-Fetal Toxicity}

Based on findings from animal studies and its mechanism of action, GAVRETO can cause fetal harm when administered to a pregnant woman. Oral administration of pralsetinib to pregnant rats during the period of organogenesis resulted in malformations and embryolethality at maternal exposures below the human exposure at the clinical dose of $400 \mathrm{mg}$ once daily.

Advise pregnant women of the potential risk to a fetus. Advise females of reproductive potential to use effective non-hormonal contraception during treatment with GAVRETO and for 2 weeks after the final dose. Advise males with female partners of reproductive potential to use effective contraception during treatment with GAVRETO and for 1 week after the final dose.

\section{ADVERSE REACTIONS}

The following clinically significant adverse reactions are described above:

- Interstitial Lung Disease/Pneumonitis

- Hypertension

- Hepatotoxicity

- Hemorrhagic Events

- Tumor Lysis Syndrome

- Risk of Impaired Wound Healing

\section{Clinical Trials Experience}

Because clinical trials are conducted under widely varying conditions, adverse reaction rates observed in the clinical trials of a drug cannot be directly compared to rates in the clinical trials of another drug and may not reflect the rates observed in practice.

The pooled safety population in the WARNINGS AND PRECAUTIONS reflect exposure to GAVRETO as a single agent at $400 \mathrm{mg}$ orally once daily in 438 patients with $R E T$-altered solid tumors, including with RET fusion-positive NSCLC $(n=220)$, and RET-altered thyroid cancer $(n=138)$, in ARROW. Among 438 patients who received GAVRETO, $47 \%$ were exposed for 6 months or longer and $23 \%$ were exposed for greater than one year.

The most common adverse reactions $(\geq 25 \%)$ were constipation, hypertension, fatigue, musculoskeletal pain 
and diarrhea. The most common Grade 3-4 laboratory abnormalities ( $\geq 2 \%$ ) were decreased lymphocytes, decreased neutrophils, decreased hemoglobin, decreased phosphate, decreased calcium (corrected), decreased sodium, increased aspartate aminotransferase (AST), increased alanine aminotransferase (ALT), decreased platelets, and increased alkaline phosphatase.

\section{RET Fusion-Positive Non-Small Cell Lung Cancer}

The safety of GAVRETO was evaluated as a single agent at $400 \mathrm{mg}$ orally once daily in 220 patients with metastatic rearranged during transfection (RET fusion-positive) non-small cell lung cancer (NSCLC) in ARROW. Among the 220 patients who received GAVRETO, $42 \%$ were exposed for 6 months or longer and $19 \%$ were exposed for greater than one year.

The median age was 60 years (range: 26 to 87 years); $52 \%$ were female, $50 \%$ were White, $41 \%$ were Asian, and $4 \%$ were Hispanic/Latino.

Serious adverse reactions occurred in $45 \%$ of patients who received GAVRETO. The most frequent serious adverse reaction (in $\geq 2 \%$ of patients) was pneumonia, pneumonitis, sepsis, urinary tract infection, and pyrexia. Fatal adverse reaction occurred in 5\% of patients; fatal adverse reaction which occurred in $>1$ patient included pneumonia $(n=3)$ and sepsis $(n=2)$.

Permanent discontinuation due to an adverse reaction occurred in $15 \%$ of patients who received GAVRETO. Adverse reactions resulting in permanent discontinuation which occurred in $>1$ patient included pneumonitis $(1.8 \%)$, pneumonia $(1.8 \%)$, and sepsis (1\%).

Dosage interruptions due to an adverse reaction occurred in $60 \%$ of patients who received GAVRETO. Adverse reactions requiring dosage interruption in $\geq 2 \%$ of patients included neutropenia, pneumonitis, anemia, hypertension, pneumonia, pyrexia, increased aspartate aminotransferase (AST), increased blood creatine phosphokinase, fatigue, leukopenia, thrombocytopenia, vomiting, increased alanine aminotransferase (ALT), sepsis, and dyspnea.

Dose reductions due to adverse reactions occurred in $36 \%$ of patients who received GAVRETO. Adverse reactions requiring dosage reductions in $\geq 2 \%$ of patients included neutropenia, anemia, pneumonitis, neutrophil count decreased, fatigue, hypertension, pneumonia, and leukopenia.

Adverse Reactions ( $\geq 15 \%$ ) in RET Fusion-Positive NSCLC Patients Who Received GAVRETO in ARROW

\begin{tabular}{|c|c|c|}
\hline \multirow[b]{2}{*}{ Adverse Reactions } & \multicolumn{2}{|c|}{ GAVRETO N=220 } \\
\hline & $\begin{array}{c}\text { Grades } 1-4 \\
(\%)\end{array}$ & $\begin{array}{c}\text { Grade 3-4 } \\
(\%)\end{array}$ \\
\hline \multicolumn{3}{|l|}{ General } \\
\hline Fatigue $^{1}$ & 35 & $2.3^{*}$ \\
\hline Pyrexia & 20 & 0 \\
\hline Edema $^{2}$ & 20 & 0 \\
\hline \multicolumn{3}{|l|}{ Gastrointestinal } \\
\hline Constipation & 35 & $1^{*}$ \\
\hline Diarrhea $^{3}$ & 24 & $3.2^{*}$ \\
\hline Dry Mouth & 16 & 0 \\
\hline \multicolumn{3}{|c|}{ Musculoskeletal Disorders } \\
\hline Musculoskeletal Pain ${ }^{4}$ & 32 & 0 \\
\hline \multicolumn{3}{|l|}{ Vascular } \\
\hline Hypertension $^{5}$ & 28 & $14^{*}$ \\
\hline \multicolumn{3}{|c|}{ Respiratory, thoracic and mediastinal } \\
\hline Cough $^{6}$ & 23 & $0.5^{*}$ \\
\hline \multicolumn{3}{|l|}{ Infections } \\
\hline Pneumonia $^{7}$ & 17 & 8 \\
\hline
\end{tabular}

1 Fatigue includes fatigue, asthenia

2 Edema includes edema peripheral, face edema, periorbital edema, eyelid edema, edema generalized, swelling

3 Diarrhea includes diarrhea, colitis, enteritis

4 Musculoskeletal pain includes back pain, myalgia, arthralgia, pain in extremity, musculoskeletal pain, neck pain, musculoskeletal chest pain, bone pain, musculoskeletal stiffness, arthritis, spinal pain

5 Hypertension includes hypertension, blood pressure increased

6 Cough includes cough, productive cough, upper-airway cough syndrome

7 Pneumonia includes pneumonia, atypical pneumonia, lung infection, pneumocystis jirovecii pneumonia, pneumonia bacterial, pneumonia cytomegaloviral, pneumonia haemophilus, pneumonia influenza, pneumonia streptococcal

*Only includes a Grade 3 adverse reaction

Select Laboratory Abnormalities ( $\geq 20 \%$ ) Worsening from Baseline in RET Fusion-Positive NSCLC Patients Who Received GAVRETO in ARROW

\begin{tabular}{|c|c|c|}
\hline \multirow{2}{*}{ Laboratory Abnormality } & \multicolumn{2}{|c|}{ GAVRETO N=220 } \\
\hline & $\begin{array}{c}\text { Grades } 1-4 \\
(\%)\end{array}$ & $\begin{array}{c}\text { Grade } 3-4 \\
(\%)\end{array}$ \\
\hline \multicolumn{3}{|l|}{ Chemistry } \\
\hline Increased AST & 74 & 2.3 \\
\hline Increased ALT & 49 & 2.3 \\
\hline Increased alkaline phosphatase & 42 & 1.8 \\
\hline Decreased calcium (corrected) & 39 & 1.8 \\
\hline Decreased albumin & 36 & 0 \\
\hline Decreased phosphate & 35 & 11 \\
\hline Increased creatinine & 33 & 0.5 \\
\hline Decreased sodium & 29 & 7 \\
\hline Increased potassium & 26 & 0.9 \\
\hline \multicolumn{3}{|l|}{ Hematology } \\
\hline Decreased neutrophils & 61 & 16 \\
\hline Decreased hemoglobin & 58 & 9 \\
\hline Decreased lymphocytes & 56 & 19 \\
\hline Decreased platelets & 27 & 3.2 \\
\hline
\end{tabular}

Denominator for each laboratory parameter is based on the number of patients with a baseline and post-treatment laboratory value available, which ranged from 216 to 218 patients.

Clinically relevant laboratory abnormalities $<20 \%$ of patients who received GAVRETO included increased phosphate (10\%).

\section{RET-altered Thyroid Cancer}

The safety of GAVRETO was evaluated as a single agent at $400 \mathrm{mg}$ orally once daily in 138 patients with RET-altered Thyroid Cancer in ARROW. Among the 138 patients who received GAVRETO, $68 \%$ were exposed for 6 months or longer, and $40 \%$ were exposed for greater than one year. The median age was 59 years (range: 18 to 83 years); $36 \%$ were female, $74 \%$ were White, $17 \%$ were Asian, and $6 \%$ were Hispanic/Latino.

Serious adverse reactions occurred in $39 \%$ of patients who received GAVRETO. The most frequent serious adverse reactions (in $\geq 2 \%$ of patients) were pneumonia, pneumonitis, urinary tract infection, pyrexia, fatigue, diarrhea, dizziness, anemia, hyponatremia, and ascites. Fatal adverse reactions occurred in $2.2 \%$ of patients; fatal adverse reactions that occurred in $>1$ patient included pneumonia $(n=2)$.

Permanent discontinuation due to an adverse reaction occurred in $9 \%$ of patients who received GAVRETO. Adverse reactions resulting in permanent discontinuation which occurred in $>1$ patient included fatigue, pneumonia, and anemia.

Dosage interruptions due to an adverse reaction occurred in $67 \%$ of patients who received GAVRETO. Adverse reactions requiring dosage interruption in $\geq 2 \%$ of patients included neutropenia, hypertension, diarrhea, fatigue, pneumonitis, anemia, increased blood creatine phosphokinase, pneumonia, urinary tract infection, musculoskeletal pain, vomiting, 
pyrexia, increased AST, dyspnea, hypocalcemia, cough, thrombocytopenia, abdominal pain, increased blood creatinine, dizziness, headache, decreased lymphocyte count, stomatitis, and syncope.

Dose reductions due to adverse reactions occurred in $44 \%$ of patients who received GAVRETO. Adverse reactions requiring dosage reductions in $\geq 2 \%$ of patients included neutropenia, anemia, hypertension, increased blood creatine phosphokinase, decreased lymphocyte count, pneumonitis, fatigue, and thrombocytopenia.

Adverse Reactions ( $\geq 15 \%$ ) in RET-altered Thyroid Cancer Patients Who Received GAVRETO in ARROW

\begin{tabular}{|c|c|c|}
\hline \multirow[b]{2}{*}{ Adverse Reactions } & \multicolumn{2}{|c|}{ GAVRETO N=138 } \\
\hline & $\begin{array}{c}\text { Grades } 1-4 \\
(\%)\end{array}$ & $\begin{array}{c}\text { Grade } 3-4 \\
(\%)\end{array}$ \\
\hline \multicolumn{3}{|l|}{ Musculoskeletal } \\
\hline Musculoskeletal Pain ${ }^{1}$ & 42 & $0.7^{*}$ \\
\hline \multicolumn{3}{|l|}{ Gastrointestinal } \\
\hline Constipation & 41 & $0.7^{*}$ \\
\hline Diarrhea $^{2}$ & 34 & $5^{*}$ \\
\hline Abdominal Pain ${ }^{3}$ & 17 & $0.7^{*}$ \\
\hline Dry Mouth & 17 & 0 \\
\hline Stomatitis $^{4}$ & 17 & $0.7^{*}$ \\
\hline Nausea & 17 & $0.7^{*}$ \\
\hline \multicolumn{3}{|l|}{ Vascular } \\
\hline Hypertension & 40 & $21^{*}$ \\
\hline \multicolumn{3}{|l|}{ General } \\
\hline Fatigue $^{5}$ & 38 & $6^{*}$ \\
\hline Edema $^{6}$ & 29 & 0 \\
\hline Pyrexia & 22 & $2.2^{*}$ \\
\hline \multicolumn{3}{|l|}{ Nervous System } \\
\hline Headache $^{7}$ & 24 & 0 \\
\hline Peripheral Neuropathy ${ }^{8}$ & 20 & 0 \\
\hline Dizziness $^{9}$ & 19 & $0.7^{*}$ \\
\hline Dysgeusia $^{10}$ & 17 & 0 \\
\hline \multicolumn{3}{|l|}{ Respiratory } \\
\hline Cough $^{11}$ & 27 & $1.4^{*}$ \\
\hline Dyspnea $^{12}$ & 22 & $2.2^{*}$ \\
\hline \multicolumn{3}{|l|}{ Skin and Subcutaneous } \\
\hline Rash $^{13}$ & 24 & 0 \\
\hline \multicolumn{3}{|c|}{ Metabolism and Nutrition } \\
\hline Decreased Appetite & 15 & 0 \\
\hline
\end{tabular}

1 Musculoskeletal Pain includes arthralgia, arthritis, back pain, bone pain, musculoskeletal chest pain, musculoskeletal pain, musculoskeletal stiffness, myalgia, neck pain, noncardiac chest pain, pain in extremity, spinal pain

2 Diarrhea includes colitis, diarrhea

3 Abdominal Pain includes abdominal discomfort, abdominal pain, abdominal pain upper, abdominal tenderness, epigastric discomfort

4 Stomatitis includes mucosal inflammation, stomatitis, tongue ulceration

5 Fatigue includes asthenia, fatigue

6 Edema includes eyelid edema, face edema, edema, edema peripheral, periorbital edema

7 Headache includes headache, migraine

8 Peripheral neuropathy includes dysaesthesia, hyperaesthesia, hypoaesthesia, neuralgia, neuropathy peripheral, paraesthesia, peripheral sensory neuropathy, polyneuropathy

9 Dizziness includes dizziness, dizziness postural, vertigo

10 Dysgeusia includes ageusia, dysgeusia

11 Cough includes cough, productive cough, upper-airway cough syndrome

12 Dyspnea includes dyspnea, dyspnea exertiona

13 Rash includes dermatitis, dermatitis acneiform, eczema, palmar-plantar, erythrodysaesthesia syndrome, rash, rash erythematous, rash macular, rash maculo-papular, rash papular, rash pustular

* Only includes a Grade 3 adverse reaction
Clinically relevant adverse reactions in $<15 \%$ of patients who received GAVRETO included tumor lysis syndrome and increased creatine phosphokinase.

Select Laboratory Abnormalities ( $\geq 20 \%$ ) Worsening from Baseline in RET-altered Thyroid Cancer Patients Who Received GAVRETO in ARROW

\begin{tabular}{|l|c|c|}
\hline \multirow{2}{*}{ Laboratory Abnormality } & \multicolumn{2}{|c|}{ GAVRETO N=138 } \\
\cline { 2 - 3 } & $\begin{array}{c}\text { Grades 1-4 } \\
(\%)\end{array}$ & $\begin{array}{c}\text { Grade 3-4 } \\
(\%)\end{array}$ \\
\hline Chemistry & 70 & 9 \\
\hline Decreased calcium (corrected) & 69 & 4.3 \\
\hline Increased AST & 43 & 3.6 \\
\hline Increased ALT & 41 & 0 \\
\hline Increased creatinine & 41 & 1.5 \\
\hline Decreased albumin & 28 & 2.2 \\
\hline Decreased sodium & 28 & 8 \\
\hline Decreased phosphate & 27 & 0.7 \\
\hline Decreased magnesium & 26 & 1.4 \\
\hline Increased potassium & 24 & 1.4 \\
\hline Increased bilirubin & 22 & 1.4 \\
\hline Increased alkaline phosphatase & \multicolumn{2}{|c|}{} \\
\hline Hematology & 67 & 27 \\
\hline Decreased lymphocytes & 63 & 13 \\
\hline Decreased hemoglobin & 59 & 16 \\
\hline Decreased neutrophils & 31 & 2.9 \\
\hline Decreased platelets & & \\
\hline
\end{tabular}

Denominator for each laboratory parameter is based on the number of patients with a baseline and post-treatment laboratory value available, which ranged from 135 to 138 patients.

Clinically relevant laboratory abnormalities in patients who received GAVRETO included increased phosphate (40\%).

\section{DRUG INTERACTIONS}

\section{Effects of Other Drugs on GAVRETO}

Strong CYP3A Inhibitors

Avoid coadministration with strong CYP3A inhibitors. Coadministration of GAVRETO with a strong CYP3A inhibitor increases pralsetinib exposure, which may increase the incidence and severity of adverse reactions of GAVRETO. Avoid coadministration of GAVRETO with combined P-gp and strong CYP3A inhibitors. If coadministration with a combined P-gp and strong CYP3A inhibitor cannot be avoided, reduce the GAVRETO dose.

\section{Strong CYP3A Inducers}

Coadministration of GAVRETO with a strong CYP3A inducer decreases pralsetinib exposure, which may decrease efficacy of GAVRETO. Avoid coadministration of GAVRETO with strong CYP3A inducers. If coadministration of GAVRETO with strong CYP3A inducers cannot be avoided, increase the GAVRETO dose.

\section{USE IN SPECIFIC POPULATIONS}

\section{Pregnancy}

Risk Summary

Based on findings from animal studies and its mechanism of action, GAVRETO can cause fetal harm when administered to a pregnant woman. There are no available data on GAVRETO use in pregnant women to inform drug-associated risk. Oral administration of pralsetinib to pregnant rats during the period of organogenesis resulted in malformations and embryolethality at maternal exposures below the human exposure at the clinical dose of $400 \mathrm{mg}$ once daily. Advise pregnant women of the potential risk to a fetus. 
In the U.S. general population, the estimated background risk of major birth defects and miscarriage in clinically recognized pregnancies is $2-4 \%$ and $15-20 \%$, respectively.

\section{Data}

\section{Animal Data}

In an embryo-fetal development study, once daily oral administration of pralsetinib to pregnant rats during the period of organogenesis resulted in $100 \%$ post-implantation loss at dose levels $\geq 20 \mathrm{mg} / \mathrm{kg}$ (approximately 1.8 times the human exposure based on area under the curve [AUC] at the clinical dose of 400 $\mathrm{mg}$ ). Post-implantation loss also occurred at the $10 \mathrm{mg} / \mathrm{kg}$ dose level (approximately 0.6 times the human exposure based on AUC at the clinical dose of $400 \mathrm{mg}$. Once daily oral administration of pralsetinib at dose levels $\geq 5 \mathrm{mg} / \mathrm{kg}$ (approximately 0.2 times the human AUC at the clinical dose of $400 \mathrm{mg}$ ) resulted in an increase in visceral malformations and variations (absent or small kidney and ureter, absent uterine horn, malpositioned kidney or testis, retroesophageal aortic arch) and skeletal malformations and variations (vertebral and rib anomalies and reduced ossification).

\section{Lactation}

\section{Risk Summary}

There are no data on the presence of pralsetinib or its metabolites in human milk or their effects on either the breastfed child or on milk production. Because of the potential for serious adverse reactions in breastfed children, advise women not to breastfeed during treatment with GAVRETO and for 1 week after the final dose.

\section{Females and Males of Reproductive Potential}

Based on animal data, GAVRETO can cause embryolethality and malformations at doses resulting in exposures below the human exposure at the clinical dose of $400 \mathrm{mg}$ daily.

\section{Pregnancy Testing}

Verify pregnancy status of females of reproductive potential prior to initiating.

\section{Contraception}

GAVRETO can cause fetal harm when administered to a pregnant woman.

\section{Females}

Advise females of reproductive potential to use effective non-hormonal contraception during treatment with

GAVRETO and for 2 weeks after the final dose. GAVRETO may render hormonal contraceptives ineffective.

\section{Males}

Advise males with female partners of reproductive potential to use effective contraception during treatment with GAVRETO and for 1 week after the final dose.

\section{Infertility}

Based on histopathological findings in the reproductive tissues of male and female rats and a dedicated fertility study in which animals of both sexes were treated and mated to each other, GAVRETO may impair fertility.

\section{Pediatric Use}

The safety and effectiveness of GAVRETO have been established in pediatric patients aged 12 years and older for RET-mutant MTC and RET-fusion thyroid cancer. Use of GAVRETO in this age group is supported by evidence from an adequate and well-controlled study of GAVRETO in adults with additional population pharmacokinetic data demonstrating that age and body weight had no clinically meaningful effect on the pharmacokinetics of pralsetinib, that the exposure of pralsetinib is expected to be similar between adults and pediatric patients aged 12 years and older, and that the course of RET-mutant MTC and RET-fusion thyroid cancer is sufficiently similar in adults and pediatric patients to allow extrapolation of data in adults to pediatric patients.

The safety and effectiveness of GAVRETO have not been established in pediatric patients with RET fusion-positive NSCLC or in pediatric patients younger than 12 years old with RET-mutant MTC or RET-fusion thyroid cancer.

\section{Animal Toxicity Data}

In a 4-week repeat-dose toxicology study in non-human primates, physeal dysplasia in the femur occurred at doses resulting in exposures similar to the human exposure (AUC) at the clinical dose of $400 \mathrm{mg}$. In rats there were findings of increased physeal thickness in the femur and sternum as well as tooth (incisor) abnormalities (fractures, dentin matrix alteration, ameloblast/odontoblast degeneration, necrosis) in both 4- and 13-week studies at doses resulting in exposures similar to the human exposure (AUC) at the clinical dose of $400 \mathrm{mg}$. Recovery was not assessed in the 13-week toxicology study, but increased physeal thickness in the femur and incisor degeneration did not show evidence of complete recovery in the 28-day rat study.

Monitor growth plates in adolescent patients with open growth plates. Consider interrupting or discontinuing therapy based on the severity of any growth plate abnormalities and based on an individual risk-benefit assessment.

\section{Geriatric Use}

Of the 438 patients in ARROW who received the recommended dose of GAVRETO at $400 \mathrm{mg}$ once daily, $30 \%$ were 65 years or older. No overall differences in pharmacokinetics (PK), safety or efficacy were observed in comparison with younger patients.

\section{Hepatic Impairment}

GAVRETO has not been studied in patients with moderate hepatic impairment (total bilirubin $>1.5$ to $3.0 \times$ upper limit of normal [ULN] and any aspartate aminotransferase [AST]) or severe hepatic impairment (total bilirubin $>3.0 \times$ ULN and any AST). No dose adjustment is required for patients with mild hepatic impairment (total bilirubin $\leq$ ULN and AST $>$ ULN or total bilirubin $>1$ to 1.5 times ULN and any AST].

Manufactured for: Blueprint Medicines Corporation,

Cambridge, MA 02139, USA

(C) 2020 Blueprint Medicines Corporation and Genentech, Inc.

All rights reserved.

FPI-0056 12/2020

For more information, go to www.GAVRETO.com or call 1-888-258-7768.

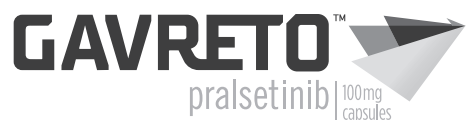




\title{
Profilin 1 Protein and Its Implications for Cancers
}

Ya Wang, MBBS; Yang Wang, MBBS; Rongjun Wan, MBBS; Chengping Hu, MD; and Yichen Lu, MD

\begin{abstract}
Profilin 1 (PFN1) is a key actin-binding protein that is involved in various cellular activities, such as cell motility, survival, and membrane trafficking. Dysfunction of PFN1 is associated with many diseases, including amyotrophic lateral sclerosis, hypertension, and cancer. Recent studies have confirmed the critical roles of PFN1 in tumorigenesis, metastasis, proliferation, and drug resistance. However, the roles of PFN1 in cancers vary among cancer types and even appear contrasting in the same cancer type in different studies. Here, we review the functions of PFN1 in cancers and discuss its potential future clinical applications. By summarizing the functions of PFN1 in cancer, we hope to better understand the mechanisms of PFN1 in cancer progression.
\end{abstract}

KEY WORDS: profilin 1; cancer; biomarker; function

\section{Introduction}

Profilin 1(PFN1) is a ubiquitoussmall-molecule protein that exists in all eukaryotes. ${ }^{1}$ PFN1 was first identified as a G-actin sequestering molecule, ${ }^{2}$ and subsequently, its true functions in actin polymerization and F-actin dynamics were revealed. ${ }^{3}$ In the following decades, the structure of PFN1 was recognized to have 3 domains: an actin-binding domain, ${ }^{4}$ a poly-L-proline (PLP)-binding domain, ${ }^{5}$ and a phosphoinositide-binding domain. ${ }^{6}$

PFN1 plays a vital role in many cell functions, including membrane trafficking, endocytosis, cell cycle, motility, proliferation, cell survival, transcription, stemness, and autophagy (Figure 1). Abnormal expression or deletion of PFN1 can affect the normal physiological activity of cells and lead to disease development. PFN1 has been deeply studied in a variety of diseases, some genetic (eg, amyotrophic lateral sclerosis) ${ }^{7}$ and some chronic (eg, hypertension). ${ }^{8}$

In the past 10 years, PFN1's role in cancer has received increasing attention. In this review, we summarize the studies of PFN1 in cancer that have been completed in recent years, discuss the roles of PFN1 in cancer, and discuss the implications for tumor diagnosis and therapy in the future.

\section{PFN1 as a biomarker for the diagnosis and prognosis of cancer}

Early diagnosis of cancers is still a major challenge worldwide, and early detection can notably reduce their associated morbidity and mortality. ${ }^{9}$ PFN1, a critical actin-binding protein, is found to be dysregulated in many cancers, which makes it possible to use it as a biomarker for diagnosis and prognosis. PFN1 mainly plays a role in the cytoplasm, but it can also be found in the nucleus and can even be secreted into the extracellular space. The rich knowledge in the proteomics field makes the detection of proteins for new diagnostic markers and targets for therapy possible. ${ }^{10}$

In some tumor types (such as renal cell carcinoma [RCC], gastric cancer, and others), high expression of PFN1 indicates later stage and worse prognosis. Via differential proteomics, PFN1 has been identified in metastatic and primary RCC, and further analysis indicated that high PFN1 expression was associated with poor outcome and that PFN1 could be used as a potential prognostic marker in RCC. ${ }^{11}$ In clear-cell RCC (ccRCC), the expression of PFN1 was decreased in early-stage tumors compared with normal tissues. However, its expression in stage IV ccRCC was significantly increased. PFN1 was selected as a candidate marker of late-stage ccRCC. ${ }^{12}$ Results of a recent study determined that the vast majority of ccRCC tumors tend to be selectively PFN1-positive in stromal cells only; dramatic transcriptional upregulation of PFN1 was found in tumor-associated vascular endothelial cells in clinical specimens of ccRCC. ${ }^{13}$ Tissue microarray 
results also showed that PFN1 was increased in metastatic ccRCC compared with primary tumors. Univariate analysis suggested that higher PFN1 expression was associated with shorter disease-free survival (HR, 7.36; $P=.047$ ) and lower overall survival. ${ }^{14}$

In gastric cancer, Tanaka et al found that PFN1 was highly expressed in fetal rat stomach. Additionally, PFN1 was overexpressed in some human and rat gastric cancers. ${ }^{15}$ The results of later studies indicated that PFN1 expression was higher in gastric cancer tissues than in adjacent normal tissues. High PFN1 expression was correlated with tumor infiltration, lymph node metastasis, and tumor-node-metastases (TNM) stage. Functional assays confirmed that silencing PFN1 could inhibit the invasion and mi- gration of gastric cancer cell lines. ${ }^{16}$

In addition, PFN1 expression was higher in non-small cell lung cancer (NSCLC). Lower expression of PFN1 was associated with better prognosis and a higher survival rate in NSCLC. ${ }^{17}$ Proteomic analysis revealed that PFN1 was differentially expressed in laryngeal carcinoma tissues compared with adjacent normal tissues. Further study results revealed that PFN1 was increased in laryngeal carcinoma tissues compared with adjacent normal tissues, indicating that PFN1 was a novel potential biomarker for the diagnosis of laryngeal carcinoma. ${ }^{18}$

However, in some other tumors (such as colorectal cancer [CRC], oral carcinoma, and others), the opposite is true. PFN1 was downregulated in pancreatic cancer. ${ }^{19-20}$ Lower expression of PFN1 was significantly associated with a shorter survival period. ${ }^{20}$ In late-stage oral squamous cell carcinoma, PFN1 expression was lower than that in normal oral epithelium, and loss of PFN1 expression was related to invasion into and metastasis of lymph nodes. ${ }^{21}$ PFN1 was also decreased in late advanced hepatocellular carcinoma (HCC) and was associated with a poor survival rate of patients. ${ }^{22-23}$ In addition, PFN1 was found to be downregulated in nasopharyngeal carcinoma ${ }^{24}$ and breast cancer. ${ }^{25}$ Combined with another 4 actin-binding proteins, PFN1 could be used to construct a model for predicting poor prognosis of esophageal squamous cell carcinoma. ${ }^{26}$

Under normal physiological conditions, PFN1 is involved in multiple cellular functions, such as cell motility, migration, ad-

\section{TABLE 1. Aberrant Expression of Profilin 1 in Various Cancers}

\begin{tabular}{|c|c|c|c|c|}
\hline Cancer type & PFN1 upregulated & PFN1 downregulated & Detection methods & Reference \\
\hline \multirow{2}{*}{$\begin{array}{l}\text { Renal cell } \\
\text { carcinoma }\end{array}$} & Metastatic tumor tissue & & Proteomics & 11 \\
\hline & Culture supernatants & & Proteomics & 34 \\
\hline \multirow[t]{2}{*}{$\begin{array}{l}\text { Clear-cell renal } \\
\text { cell carcinoma }\end{array}$} & Late stage (stage III-IV) tumor tissue & & $\begin{array}{l}\text { Proteotranscriptomic } \\
\text { analysis }\end{array}$ & 12 \\
\hline & Metastatic tumor tissue & & Tissue microarray & 14 \\
\hline \multirow[t]{2}{*}{ Gastric cancer } & Rat gastric tumor tissue & & PCR & 15 \\
\hline & Tumor tissue & & IHC; WB; PCR & 16 \\
\hline \multirow[t]{2}{*}{ Gallbladder cancer } & Serum & & Proteomics & 31 \\
\hline & Urine & & Proteomics & 32 \\
\hline \multirow[t]{2}{*}{ HCC } & Peripheral blood cells of patients with HCC & & Gene chip and PCR & 29,30 \\
\hline & & Tumor tissue/cell lines & IHC; WB & 22,23 \\
\hline \multirow[t]{2}{*}{ Breast cancer } & & Cell lines & $\begin{array}{l}\text { Differential display } \\
\text { screening }\end{array}$ & 25 \\
\hline & & $\begin{array}{l}\text { Circulating leukocytes } \\
\text { of patients with cancer }\end{array}$ & $\begin{array}{l}\text { Differential display } \\
\text { screening }\end{array}$ & 35 \\
\hline Pancreatic cancer & & Tumor tissue & $\begin{array}{l}\text { Array comparative } \\
\text { genomic hybridization; } \\
\text { tissue microarray }\end{array}$ & 19,20 \\
\hline Pancreatic cancer & & Secretome of cell lines & Proteomics & 33 \\
\hline $\begin{array}{l}\text { Oral squamous } \\
\text { cell carcinoma }\end{array}$ & & Tumor tissue & $\mathrm{IHC}$ & 21 \\
\hline $\begin{array}{l}\text { Nasopharyngeal } \\
\text { carcinoma }\end{array}$ & & Cell lines & Proteomics & 24 \\
\hline
\end{tabular}

HCC, hepatocellular carcinoma; IHC, immunohistochemistry; IV, intravenous; PCR, polymerase chain reaction; PFN1, profilin 1; WB, Western blot. 
hesion, and transduction signaling pathways. ${ }^{27}$ PFN1 is differentially expressed in various types of tissues and cells, which may explain its variable tumorigenic mechanisms in different tumors, even in different stages of the same cancer (Figure 2). Because PFN1 plays important roles in tumorigenesis and progression, targeting PFN1 dysregulation could to some extent influence the prognosis of patients with cancer. Determining the expression of PFN1 could thus be used to distinguish high-risk disease from lower-risk disease. Combination with other indices could further improve the diagnostic and prognostic value of PFN1.

In addition to dysregulation in tumor tissues, PFN1 was also found to be differentially expressed in the serum, urine, and extracellular vesicles of patients with cancer, which makes it possible to utilize PFN1 in liquid biopsy analysis of tumors. Compared with tumor tissue biopsy, liquid biopsy is a more practical method for real-time monitoring of patients with cancer. ${ }^{28}$ In addition, PFN1 was detected in the supernatants of cultured cells.

It has been shown that PFN1 gene expression is increased in peripheral blood cells of patients with HCC compared with healthy controls. ${ }^{29} \mathrm{~A}$ 9-gene expres- sion system (including PFN1) was used to discriminate patients with HCC from healthy people. ${ }^{30}$ Proteomic analysis of serum proteins showed that PFN1 was increased in patients with gallbladder cancer. The expression difference between these patients and healthy controls was more than 2-fold. ${ }^{31}$ PFN1 was differentially expressed in the urine of patients with invasive and noninvasive bladder cancer. Further studies confirmed that PFN1 was notably decreased in the epithelium of invasive bladder tumors compared with noninvasive tumors, which was associated with the clinical outcomes of bladder cancer. ${ }^{32}$ In in vitro pancreatic cancer cell lines, PFN1 was downregulated in secretomes compared with nonneoplastic pancreatic ductal cells. ${ }^{33}$ In in vitro cultured RCC cell lines, PFN1 was differentially regulated in the supernatant. Further studies revealed that PFN1 was upregulated in RCC tissues. ${ }^{34}$ Apart from its dysregulation in serum and urine, PFN1 was found to be downregulated in the circulating leukocytes of patients with breast cancer compared with healthy controls, which provides a new paradigm for highly sensitive and less invasive approaches for the diagnosis of breast cancer. ${ }^{35}$ Studies have already revealed that PFN1 can be secreted via exosomes or other secretory pathways. ${ }^{36-38}$

Extracellular PFN1 in the tumor microenvironment can be taken up by recipient cells and execute its function in recipient cells, which in turn may influence the biological behavior of cells in the microenvironment, ultimately affecting tumorigenesis and progression of cancers. As mentioned above, PFN1 is expressed differentially in the serum and urine of patients with cancer, which enables its application as a biomarker for diagnosis and prognosis in liquid biopsy (Table 1).

\section{PFN1 as a promoter or inhibitor of cancer metastasis}

Cell motility involves membrane protrusion, cell matrix adhesion, cell body translocation, and rear detachment. Many of these processes require the actin cytoskeleton and its regulators. By facilitating the exchange of ATP for ADP on G-actin, PFN1 plays a major role in actin polymerization, thus influencing motility in numerous cells. ${ }^{39}$ PFN1 also participates in cell motility by regulating actin polymerization and interactions with other regulators of actin cytoskeletons, such as ARP3, VASP, and proteins of cell signaling path-

\section{FIGURE 1. Functions of Profilin 1 in Cancer}

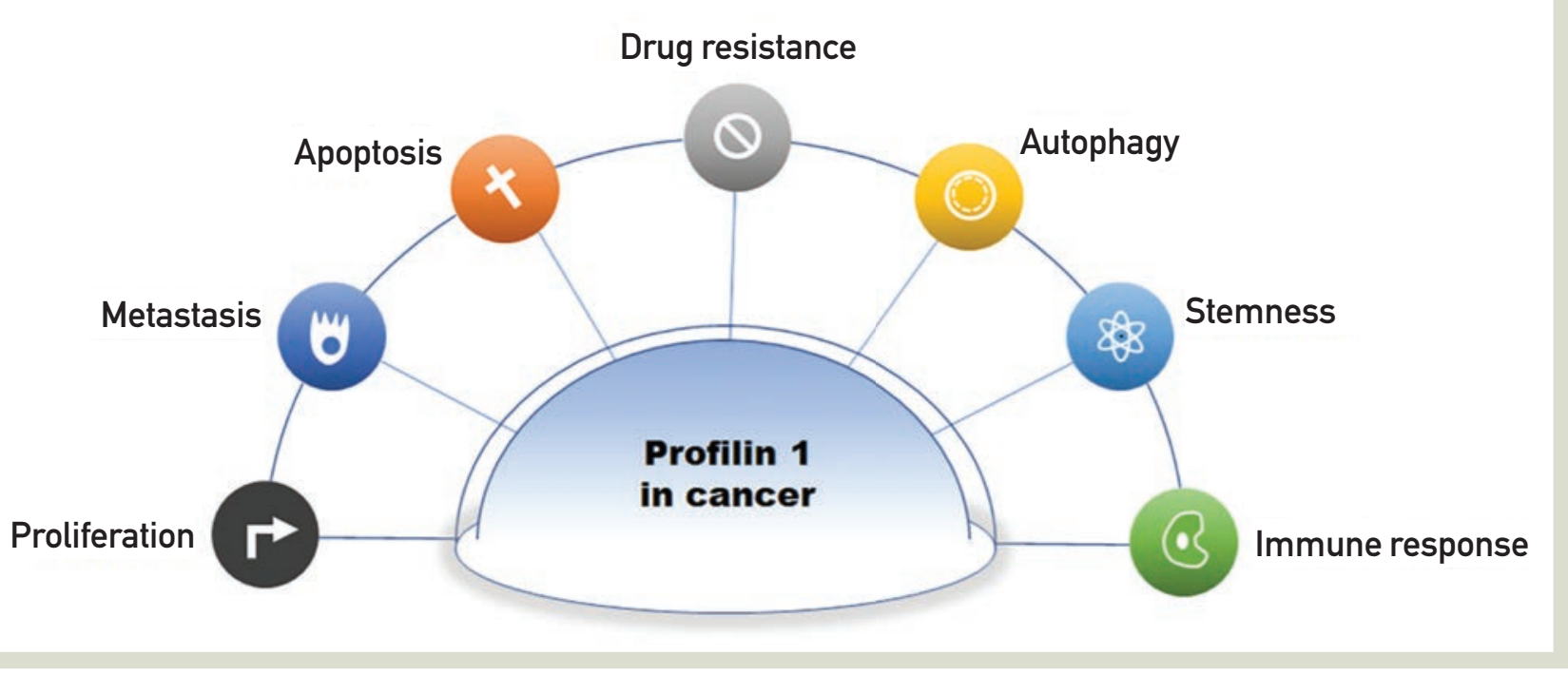




\section{TABLE 2. Mechanisms of PFN1 in Metastasis of Different Cancers}

\begin{tabular}{|c|c|c|c|c|}
\hline Cancer type & $\begin{array}{l}\text { Inhibitor of } \\
\text { metastasis }\end{array}$ & $\begin{array}{l}\text { Promoter of } \\
\text { metastasis }\end{array}$ & Underlying mechanisms & Reference \\
\hline \multirow[t]{7}{*}{ Breast cancer } & $\sqrt{ }$ & & Tyrosine phosphorylation of FAK and paxillin & 49 \\
\hline & $\sqrt{ }$ & & $\begin{array}{l}\text { Induces actin stress-fibers, upregulates focal adhesion, and } \\
\text { inhibits motility and invasiveness }\end{array}$ & 50 \\
\hline & $\sqrt{ }$ & & $\begin{array}{l}\text { PFN1 downregulation results in a hypermotile phenotype of } \\
\text { MDA-MB-231 cells in an Ena/VASP-dependent mechanism. }\end{array}$ & 51 \\
\hline & $\sqrt{ }$ & & Negatively regulated by miRNA-182 & 52 \\
\hline & $\sqrt{ }$ & & $\begin{array}{l}\text { Knockdown of PFN1 abrogated the inhibitory effect of tyrphostin } \\
\text { A9 on cell migration. }\end{array}$ & 59 \\
\hline & $\sqrt{ }$ & & Interacts with and ubiquitinates PFN1 & 53 \\
\hline & & $\sqrt{ }$ & $\begin{array}{l}\text { Phosphorylation of PFN1 at serine } 137 \text { enhances oncogenic } \\
\text { properties. }\end{array}$ & 64 \\
\hline Bladder cancer & & $\sqrt{ }$ & Noncanonical Wnt/Ca2+ signaling pathway & 66 \\
\hline Lung cancer & & $\sqrt{ }$ & NA & 17 \\
\hline Colorectal cancer & & $\sqrt{ }$ & Regulated by HLA-F-AS1 & 62 \\
\hline $\begin{array}{l}\text { Hepatocellular } \\
\text { carcinoma }\end{array}$ & $\sqrt{ }$ & & Negatively regulated by microRNA-19a-3p & $22,23,60$ \\
\hline Prostate cancer & $\sqrt{ }$ & & $\begin{array}{l}\text { Inactivation of the tumor suppressive function of PFN1 by } \\
\text { cathepsin X }\end{array}$ & 61 \\
\hline Gastric cancer & & $\sqrt{ }$ & Integrin $\beta 1 / F A K$ pathway & 16 \\
\hline
\end{tabular}

Ena/VASP, Enabled/vasodilator stimulated phosphoprotein; FAK, focal adhesion kinase; NA, not applicable; PFN1, profilin 1.

ways. Cell-cell adhesion and cell-matrix adhesion are critical contributors to maintaining tissue architecture. Dysregulation of cell-cell adhesion is an important sign in tumor initiation and progression of malignancy. PFN1 can modulate cell adhesion and epithelial-to-mesenchymal transition (EMT) in cancer cells. However, the mechanisms by which PFN1 regulates cell adhesion are still not very clear. Undoubtedly, learning more about the roles of PFN1 in cell adhesion and motility will help us better understand its roles in modulating tumor invasion and migration.

Since PFN1 plays a critical role in actin polymerization, it is an indispensable regulator of cell motility. PFN1 participates in the invasion and metastasis of multiple cancers. However, the roles of PFN1 in regulating cell motility are context specific. ${ }^{27}$ Exogenous PFN1 with intact actin-binding abilities can ameliorate the adherence and spreading capabilities of cancer cells and exert tumor-suppressive effects in breast cancer. ${ }^{40}$ Consistent with the results of the study by Wittenmayer et al, Zou et al found that PFN1 overexpression could revert MDA MB-231 cells to an epithelioid phenotype, with restored adherence junctions. ${ }^{41}$ In addition, PFN1 overexpression could promote AMPK activation and p27 phosphorylation, which in turn induces epithelial morphological reversion of mesenchymal breast cancer through restoration of adherens junctions. ${ }^{42}$ These studies highlighted the involvement of PFN1 in epithelial adhesion and differentiation, which helped us better understand its roles in cancer cell motility.

Invadopodia are actin-driven membrane protrusions that can deliver matrix metalloproteinases to degrade the matrix and support invasion and dissemination of tumor cells. Any dysregulation of the actin cytoskeleton can impair the formation and maturation of invadopodia. ${ }^{43-46}$ PFN1 can regulate $\mathrm{PI}(3,4) \mathrm{P} 2$, which in turn negatively regulates lamellipodin at the leading edge of breast cancer cells and thus inhibits those cells' motility. ${ }^{47}$ The depletion of PFN1 leads to an increase in the level of $\mathrm{PI}(3,4) \mathrm{P} 2$ in invadopodia and its interacting adaptor Tks5. The interaction of PI(3,4)P2-Tks5 has been shown to promote the anchorage, maturation, and turnover of invadopodia, which in turn enhances the invasiveness and motility of breast cancer. ${ }^{48}$ Breast cancer is an invasive adenocarcinoma, and numerous studies have found that PFN1 is downregulated in breast cancer tissues. ${ }^{49-54}$ Overexpression of PFN1 reduces the invasion and migration of breast cancer cells, while loss of PFN1 significantly enhances breast cancer cell motility and invasion. Mechanisms involved in PFN1's negative roles in breast cancer metastasis include Enabled (Ena)/vasodilator stimulated phosphoprotein (VASP)-dependent lamellipodial protrusion, ${ }^{51}$ miRNA-182 regulation, ${ }^{52}$ and regulation of PFN1 degradation. ${ }^{53}$ 
Mouneimne et al found that PFN1 knockdown (KD) could increase F-actin bundles and enhance stress fiber formation. by the HLA-F-AS1/miRNA-330-3p/ PFN1 or HCP5/miRNA-299-3p/PFN1/ AKT axis. ${ }^{62-63}$

\section{PFN1 participates in multiple biological processes of tumor development and progression [and] plays opposite roles in different tumors and at different periods of tumor, potentially leading to the conclusion that PFN1's function in tumor has spatial and temporal specificity.}

In that study, the numbers of protrusions in PFN1-KD cells were markedly decreased, and PFN1-KD could inhibit the motility of breast cancer. ${ }^{55}$ Moreover, Liu et al indicated that the interaction of LMO2PFN1 and LMO2-ARP3 could promote the formation of lamellipodia/filopodia in basal-type breast cancer cells. ${ }^{56}$ Ena/ VASP is a critical regulator of the actin cytoskeleton at the leading edge of cells, which controls membrane protrusions and cell motility. Cell-substrate adhesion and downregulation of Protein Kinase A (PKA) promote interactions of PFN1 with VASP, which is another mechanism by which PFN1 regulates cell motility. ${ }^{57-58}$ Knockdown of PFN-1 has been shown to abrogate the inhibitory effect of tyrphostin A9, suggesting that modulating PFN1 expression could have therapeutic potential in the treatment of metastatic breast cancer. ${ }^{59}$

As in breast cancer, PFN1 was found to be a suppressor of migration in HCC. ${ }^{22,23,60}$ All-trans retinoic acid $^{60}$ and guttiferone $\mathrm{K}^{22}$ could inhibit hepatocellular cell migration and proliferation by upregulating the expression of PFN1. In prostate cancer, cathepsin X can inactivate PFN1, thus promoting adhesion, invasion, and migration of cancer cells. ${ }^{61}$ In CRC, elevated expression of PFN1 obviously inhibited invasion and migration. PFN1 was suppressed
Interestingly, Ding et al showed that in the early stages of metastasis, breast cancer cells exhibit a hyperinvasive phenotype characterized by upregulation of MMP-9 and by faster invasion when PFN1 expression is downregulated. However, in the late stages of metastasis, loss of PFN1 markedly inhibits the growth of metastatic colonies of breast cancer cells. ${ }^{54}$ Rizwani et al reported that PFN1 expression was elevated in breast cancer tissues and that overexpression of PFN1 could inhibit the migration of breast cancer cells. The phosphorylation of S137 mutants abrogated PFN1's promotion of migration. These studies provided a different vision of PFN1's role in breast cancer metastasis. ${ }^{64}$

In gastric cancer, silencing PFN1 inhibited the invasion and migration of cells, and the PFN1 expression level in cancer tissue was positively correlated with tumor infiltration and lymph node metastasis. ${ }^{16}$ However, different conclusions were drawn from the study of Ma et al. The authors found that PFN1 expression was inversely correlated with lymph node metastasis. ${ }^{65}$ In the lung cancer cell line A549, downregulation of PFN1 inhibited migration. ${ }^{17}$ In addition, in vitro studies support the importance of PFN1 in the proliferation and migration of RCC cells, and treatment with a novel computationally designed PFN1-actin interaction inhibitor reduced the proliferation and migration of RCC cells in vitro and RCC tumor growth in vivo. ${ }^{13}$ Additional studies have demonstrated that downregulation of PFN1 can also suppress the migration of laryngeal cancer $^{18}$ and bladder cancer. ${ }^{66}$

Although more studies on PFN1 have been completed recently, its roles in cancer metastasis are still unclear. The concentrations of actin and PFN1 are time- and space-specific, and so is the regulation of the actin cytoskeleton (Table 2). Additional thorough studies are needed to comprehend the mechanisms and laws regulating the actin cytoskeleton. More importantly, in addition to actin dependence, PFN1 affects cell migration in an actin-independent manner by interacting with proteins with PIP2 or PLP domains. Furthermore, lncRNAs and microRNAs also modulate the functions of PFN1. All of these proteins and RNAs interact with PFN1 and indirectly influence the functions of cancer cells, which makes understanding the roles of PFN1 in cancer metastasis and other functions more complicated (Table 3).

\section{PFN1 as a regulator of proliferation, apoptosis, and drug resistance in cancer}

In yeast, the gene encoding PFN1 is essential for cytokinesis. ${ }^{67}$ Early studies revealed that PFN1-/- embryos died as early as the 2-cell stage, while PFN1-/+embryos displayed reduced survival during embryogenesis compared with wildtype embryos; this indicates that PFN1 is essential for cell division and survival during embryogenesis. ${ }^{68}$ PFN1 silencing in endothelial cells inhibits proliferation. ${ }^{69}$ In addition, homozygous deletion of PFN1 in chondrocytes failed to complete abscission at late-stage cytokinesis. ${ }^{70}$ The results of all these studies imply that PFN1 plays a role in cell proliferation. In breast cancer, PFN1 overexpression (PFN1-OE) has been shown to inhibit cell growth and exert an inhibitory effect 
on tumorigenesis, ${ }^{25,40,52,71-75}$ and PFN1OE suppresses the activation of AKT, which in turn inhibits the growth of tumor cells. ${ }^{71}$ PFN1-OE cells arrested at the G1 phase, which was partly attributed to the upregulation of P2 $7^{\text {kip } 1.72}$ miRNA-182 could downregulate PFN1 expression and promote triple-negative breast cancer cell proliferation. ${ }^{52}$ However, Yap et al put forward opposite views. The authors' research results revealed that silencing PFN1 resulted in a multinucleation phenotype of breast cancer cells, thus inhibiting proliferation. ${ }^{76}$ Recent studies from Chakraborty et al also reported that PFN1 knockdown could upregulate SMAD3 and inhibit the proliferation of breast cancer. ${ }^{77}$ Results of single-cell studies on the extracellular matrix revealed that stiff extracellular matrix led to upregulation of PFN1, possibly promoting the proliferation of breast cancer. ${ }^{78}$ Apart from breast cancer, PFN1 was also found to suppress proliferation in pancreatic adenocarcinoma, ${ }^{20}$ endometrial cancer, ${ }^{79}$ and HCC. ${ }^{23,60}$ In gastric cancer, silencing PFN1 caused cell cycle arrest at G0/ G1 phase, thus restraining cell proliferation. ${ }^{16}$ Knockdown of PFN1 could also inhibit the proliferation of laryngeal cancer. ${ }^{18}$ Our previous studies found that overexpression of PFN1 could promote the proliferation of multiple myeloma cells by accelerating the cell cycle from G1 to $S$ phase.$^{80}$ PFN1 is indispensable for cytokinesis. Nevertheless, PFN1 is involved in regulating cell proliferation not only by impacting cytokinesis but also by modulating cell cycle-related proteins. Otherwise, PFN1 could also interact with cell signaling pathways and indirectly influence cell proliferation.

Tumor growth is not only about uncontrolled proliferation but also resistance to apoptosis. ${ }^{81}$ Actin dynamics have notable impacts on multiple stages of apoptosis. ${ }^{82} \mathrm{PFN} 1$, as a critical actin-binding protein, is an indispensable regulator of actin dynamics, through which PFN1 participates in regulating apoptosis. PFN1 overexpression could upregulate the most common tumor-associated hotspot mutation of p53-p53R273H-thus sensitizing cancer cells to apoptosis via the intrinsic apoptotic pathway. ${ }^{83}$ PFN1 has been shown to facilitate apoptosis of breast cancer cells, thus exerting a suppressive effect on tumorigenesis. ${ }^{73,75,83,84}$
By inducing apoptosis and reducing autophagy, PFN1 has also been shown to sensitize pancreatic cancer cells to irradiation. Additionally, overexpression of PFN1 can significantly elevate apoptotic markers such as cleaved caspase- 3 and cleaved PARP after irradiation, suggesting that PFN1 can modulate radiosensitivity partly by regulating apoptosis. ${ }^{85}$

Given that PFN1 is involved in cell proliferation and apoptosis, it is not difficult to understand its roles in the drug resistance of tumor cells. PFN1 was found to be downregulated in butyrate-treated CRC cells, ${ }^{86}$ and proteomics studies revealed that PFN1 was differentially expressed in erinacine A-treated CRC cells, ${ }^{87}$ which suggested the roles of PFN1 in drug-mediated cell death and inhibition of proliferation. In addition, proteomics showed that PFN1 was differentially expressed in mitotane-treated adrenocortical carcinoma, ${ }^{88}$ and PFN1 was found to be increased in tocotrienol-treated MDA-MB-231 cells, ${ }^{89}$ indicating its roles in predicting the response to anticancer therapies. Compared with temozolomide (TMZ)-treated glioblastoma cells, PFN1 was downregulated

\section{FIGURE 2. Two Sides of Profilin 1 in Different TumorTypes}

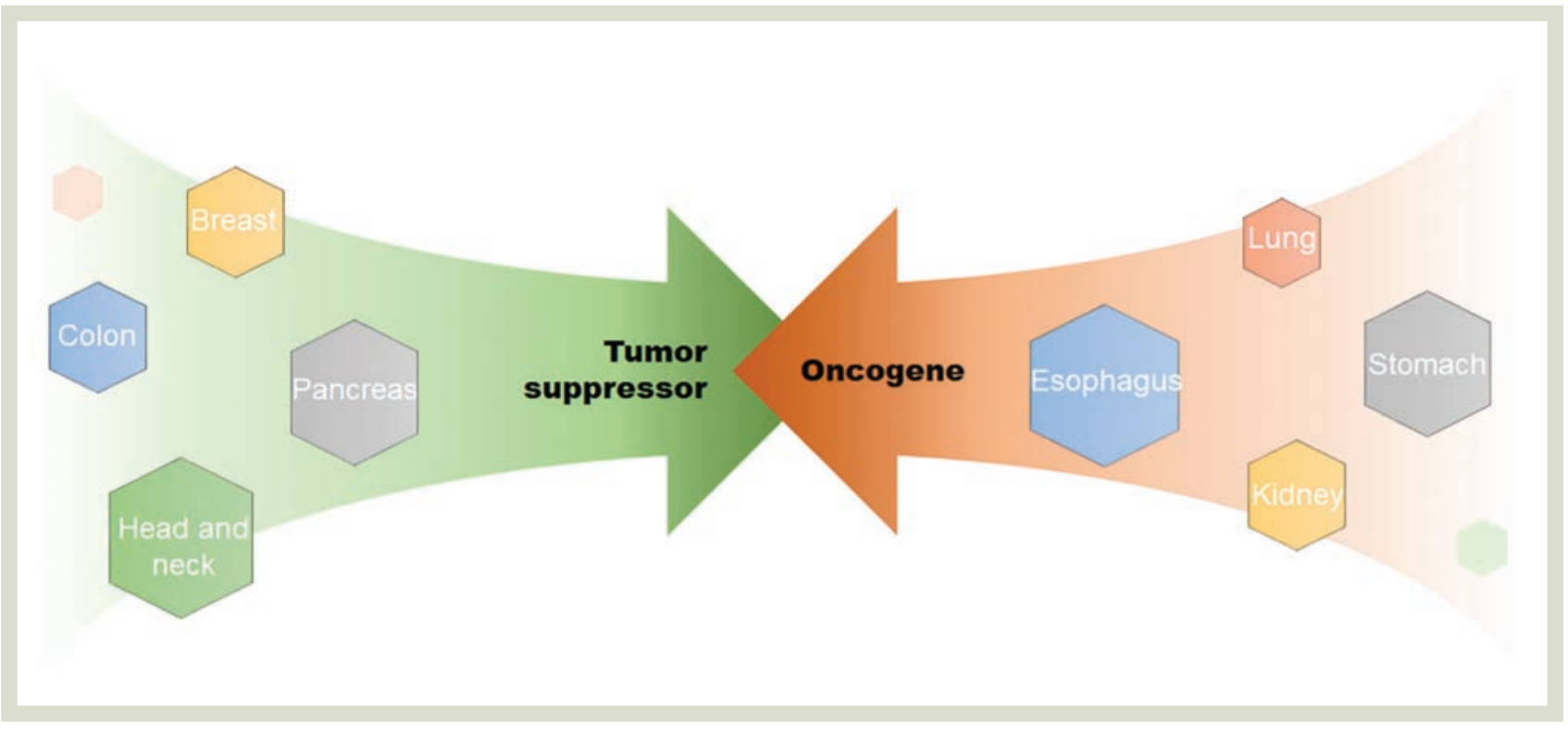




\section{TABLE 3. Signaling Pathways or Proteins That Interact With PFN1}

\begin{tabular}{|c|c|c|c|}
\hline Signaling pathway/protein & Relationship with PFN1 & Functions & Reference \\
\hline $\begin{array}{l}\text { Focal adhesion kinase } \\
\text { and paxillin }\end{array}$ & $\begin{array}{l}\text { Overexpression of PFN1 increases } \\
\text { tyrosine phosphorylation }\end{array}$ & Resistance to deatachment & 49 \\
\hline Ena/VASP proteins & $\begin{array}{l}\text { Loss of PFN1 increases membrane } \\
\text { recruitment }\end{array}$ & Promotion of motility & 51 \\
\hline R-cadherin & $\begin{array}{l}\text { Overexpression of PFN1 increases } \\
\text { expression }\end{array}$ & Regulation of epithelial cell-cell adhesion & 41 \\
\hline PTEN and AKT & $\begin{array}{l}\text { Overexpression of PFN1 causes PTEN } \\
\text { upregulation and decreased activation } \\
\text { of AKT }\end{array}$ & Tumor suppression & 71 \\
\hline p27kip1 & PFN1 overexpression increases stability & Tumor suppression & 72 \\
\hline Phosphoinositide & Interaction with PFN1 & Inhibiting motility & 47 \\
\hline p53 R273H & Is upregulated by PFN1 overexpression & Potentiating apoptosis & 83 \\
\hline $\begin{array}{l}\text { C-terminus of Hsc70- } \\
\text { interacting protein }\end{array}$ & Interacts with and ubiquitinates PFN1 & Enhanced cell migration & 53 \\
\hline HLA-F-AS1 & Regulates expression of PFN1 & $\begin{array}{l}\text { Promoting the proliferation, migration, } \\
\text { invasion, and blocking apoptosis of CRC cells }\end{array}$ & 62 \\
\hline miRNA-19a-3p & Regulates expression of PFN1 & $\begin{array}{l}\text { Inhibiting the proliferation, migration, invasion, } \\
\text { and EMT of HCC cells }\end{array}$ & 23 \\
\hline VASP & Interacts with PFN1 & Cell motility and metastasis & 57,58 \\
\hline miRNA-182 & Negatively regulates PFN1 expression & $\begin{array}{l}\text { Promoting proliferation and invasion of MDA- } \\
\text { MB-231 cells }\end{array}$ & 52 \\
\hline Cathepsin X & $\begin{array}{l}\text { PFN1 is a target of cathepsin } \mathrm{X} \text { car- } \\
\text { boxypeptidase activity }\end{array}$ & $\begin{array}{l}\text { Promoting adhesion, migration, and } \\
\text { invasiveness }\end{array}$ & 61 \\
\hline $\operatorname{ER} \alpha$ & Interacts with PFN1 & $\begin{array}{l}\text { PFN1 is a putative corepressor of ER } \alpha \\
\text { functions }\end{array}$ & 73 \\
\hline SIRT3-HIF1 $\alpha$ axis & PFN1 upregulates SIRT3 & Tumor suppression & 20 \\
\hline $\begin{array}{l}\text { Noncanonical Wnt/Ca2+ } \\
\text { signaling pathway }\end{array}$ & $\begin{array}{l}\text { PFN1 suppression could induce de- } \\
\text { crease of key proteins of this pathway }\end{array}$ & Suppresses cell adhesion and tumor growth & 66 \\
\hline SMAD3 & $\begin{array}{l}\text { PFN1 deficiency leads to SMAD3 } \\
\text { upregulation }\end{array}$ & Reduces metastatic outgrowth & 77 \\
\hline
\end{tabular}

EMT, epithelial-to-mesenchymal transition; Ena/VASP, Enabled/vasodilator stimulated phosphoprotein; ER $\alpha$, estrogen receptor alpha; FAK, focal adhesion kinase; miRNA, microRNA; PFN1, profilin 1.

in OKN-007 combined with TMZ-treated glioblastoma cells. Further study results revealed that PFN1 is involved in TMZ resistance. ${ }^{90}$ Results of our previous studies showed that PFN1 could interact with the Beclin 1 complex and participate in bortezomib resistance in multiple myeloma. ${ }^{80}$ Since PFN1 is involved in multiple cell processes, including proliferation, apoptosis, and proteomics, it was recognized as a biomarker for therapy sensitivity, and it is worth further exploring its roles in drug resistance. In addition, PFN1 was found to participate in angiogenesis, ${ }^{91-92}$ initiation of tumors, ${ }^{93}$ and autophagy. ${ }^{80}$ Loss of PFN1 in A549 cell lines resulted in fewer early apoptotic cells after treatment with piperlongumine, and PFN1 sensitized A549 cells to anticancer agents. ${ }^{17}$ PFN1 serves as a bridge for actin-cytoskeleton and cell signaling pathways and is involved in multiple biological and physiological processes. Dysregulation of PFN1 in cancer cells has a notable impact on sensitivity to chemotherapy or radiotherapy and may be a new target for the treatment of drug-resistant or radioresistant patients.

\section{Other functions of PFN 1 in tumors}

Studies have already confirmed that PFN1 is essential for cell survival in early embryos, as PFN1-KN could induce Drosophila embryos to die at the 2 -cell stage. ${ }^{94}$ 
For further investigation of PFN1's roles in tissue-specific stem cells, Zheng et al established PFN1flox/flox mice that inducibly delete PFN1 in HSCs. Results showed that PFN1 was essential for the retention and metabolism of mouse hematopoietic stem cells in bone marrow partially through the axis of PFN1/ Ga13/EGR1. ${ }^{95}$ These study results implied important roles of PFN1 in stem cell function, which were still unclear and deserved further research. Later study results have found that both overexpression and depletion of PFN1 could reduce the stem-like phenotype of MDA-MB-231 (MDA-231) triple-negative breast cancer cells, suggesting that a balanced expression of PFN1 was required for maintenance of optimal stemness and tumor-initiating ability of breast cancer cells. ${ }^{93}$ Considering that tumor heterogeneity is still an ongoing challenge for cancer treatment and that cancer stem cells (CSC) are considered to be a determining factor of tumor heterogeneity, ${ }^{96}$ intensive studies on PFN1's roles in CSC may provide us new insight into tumor initiation.
As mentioned above, PFN1 has been shown to be a critical participator of actin dynamics and to play important roles in cell migration. For cytotoxic T lymphocytes (CTLs), migration abilities are essential for patrolling tissues and locating targeted cells. ${ }^{97-98}$ Schoppmeyer et al thus studied PFN1's roles in CTL functions. The authors found that PFN1 negatively regulated CTL-mediated elimination of target cells and that PFN1 downregulation promoted CTL invasion into a 3D matrix in vitro. In patients with pancreatic cancer, PFN1 expression was substantially decreased in peripheral CD8+ T cells. ${ }^{99}$ However, considering the complexity of immune responses in vivo, the exact roles of PFN1 in tumor immunity remain unclear and need to be further explored.

\section{Conclusions}

Based on previous studies, we found that PFN1 participates in multiple biological processes of tumor development and progression. Meanwhile, it is noteworthy that PFN1 plays opposite roles in different tumors and at different periods of tumor, potentially leading to the conclusion that PFN1's function in tumor has spatial and temporal specificity. Future studies on PFN1 should take this into account. PFN1 was shown to be of great significance for diagnosis and prognosis prediction and for monitoring the therapeutic effect of anticancer drugs, and PFN1's roles in tumor stemness and immunity may provide a new avenue for cancer therapy. Although much research has been done on PFN1 and cancer, puzzles still need to be solved. With deepening research, the function of PFN1 in cancer would be further clarified and its clinical value would be more prominent.

FINANCIAL DISCLOSURE: The authors have no significant financial interest in or other relationship with the manufacturer of any product or provider of any service mentioned in this article.

CONFLICTS OF INTEREST: Authors declare no conflicts of interest for this article.

ACKNOWLEDGMENT: The authors are thankful for financial support from the Doctoral Fund Project of Hunan Provincial People's Hospital (program number BSJJ201812).

For full reference list, visit cancernetwork.com/Wang_7.21

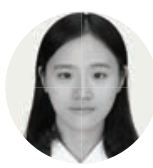

\section{Wang}

is from the Department of Respiratory Medicine in the Department of Respiratory and Critical Care Medicine, National Key Clinical Specialty at Xiangya Hospital of Central South University in Hunan, China.

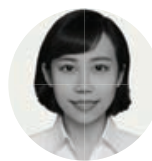

Wang

is from the Department of Respiratory Medicine in the Department of Respiratory and Critical Care Medicine, National Key Clinical Specialty at Xiangya Hospital of Central South University in Hunan, China.

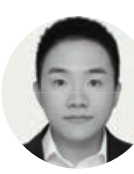

\section{Wan}

is from the Department of Respiratory Medicine in the Department of Respiratory and Critical Care Medicine, National Key Clinical Specialty at Xiangya Hospital of Central South University in Hunan, China.

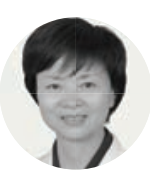

\section{Hu}

is from the Department of Respiratory Medicine in the Department of Respiratory and Critical Care Medicine, National Key Clinical Specialty at Xiangya Hospital of Central South University in Hunan, China.

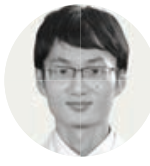

\section{Lu}

if from the Department of Oncology in the Hunan Provincial People's Hospital/ The First Affiliated Hospital of Hunan Normal University in Changsha, Hunan, PR China 


\section{Combination Therapy With Immune Checkpoint Inhibitors in Urothelial Carcinoma: Current Data and Future Outlook}

Zizhen Feng, MD, PhD; and Jacqueline Vuky, MD

ABSTRACT: Until 5 years ago, systemic therapeutic options for urothelial carcinoma, the most common form of bladder cancer, had been limited to cisplatin-based regimens (methotrexate/vinblastine/doxorubicin/cisplatin; gemcitabine/cisplatin) and taxanes (as single agents or in combinations), which had minimal survival benefit and excessive toxicity. In 2016, systemic options expanded with the advent of immunotherapy oncology (IO) drug approvals. Due to the different and lower toxicity profiles of these 10 agents, their approvals in the first-line platinum-ineligible (pembrolizumab [Keytruda]; atezolizumab[Tecentriq]) and second-line metastatic (avelumab [Bavencio]; atezolizumab; durvalumab [Imfinzi]; nivolumab [Opdivo]; pembrolizumab) settings expanded our ability to treat a broader population of patients with metastatic disease. However, this came with the realization that response rates were low and, although there were durable responses, there was also a lack of predictive markers to select which patients would benefit. With several recent approvals-of the first biomarker-targeted therapy to treat urothelial carcinoma, erdafitinib (Balversa), and of the antibody-drug conjugates enfortumab vedotin (EV; Padcev) and sacituzumab govitecan-we are now challenged with sequencing and exploring combination therapies with IOs. This article explores the current and future outlooks for combination therapy with IOs in urothelial carcinoma.

\section{Introduction}

Bladder cancer is the sixth most common cancer in the United States, with an estimated 81,400 new cases in 2020. Although bladder cancer has 4 stages, for systemic treatment we recognize 3 clinical stages: non-muscle-invasive bladder cancer (NMIBC), muscle-invasive bladder cancer (MIBC), and locally advanced/metastatic urothelial carcinoma (mUC). Approximately $70 \%$ to $80 \%$ of patients present with NMIBC at diagnosis and have an excellent 5 -year overall survival (OS) of $69.2 \%$ to $95.8 \%$. ${ }^{1}$ About $10 \%$ to $15 \%$ of patients present with MIBC at the time of diagnosis and have about a $50 \%$ chance of progressing to metastatic disease. ${ }^{2} \mathrm{mUC}$ accounts for $10 \%$ to $15 \%$ of all bladder cancers at diagnosis, with a 5 -year OS for patients of fewer than $10 \%$ with platinum-based chemotherapy.

\section{1. mUC}

Historically, mUC is considered a chemotherapysensitive disease with response rates of $50 \%$ to $60 \% .^{3-5}$ However, cisplatin-based regimens (eg, methotrexate/vinblastine/doxorubicin/cisplatin [MVAC]; gemcitabine/cisplatin) result in a high rate of complications, including nephrotoxicity, ototoxicity, neurotoxicity, and cardiotoxicity, and about half of patients are ineligible to receive cisplatin-based therapy due to renal insufficiency, poor performance status, or preexisting neurotoxicity and hearing loss. Median OS (OS) for patients with mUC is poor, at 13.8 to 14.8 months. ${ }^{5}$

In 2017, after promising results from the phase 


\section{KEYNOTE-052 (NCT02335424)} and phase 2 IMvigor210 trials (NCT02108652), the FDA granted accelerated approval to 2 immune checkpoint inhibitors (ICIs), pembrolizumab and atezolizumab, as first-line treatment for patients with locally advanced or metastatic UC who were not eligible for platinum-based therapy. ${ }^{6-8}$ Both trials enrolled similar patients; they were cisplatin ineligible and treatment naïve in the metastatic setting. The results of both trials demonstrated comparable response rates of $23 \%$ to $29 \%$ (Table 1 ). In the KEYNOTE-052 trial, the median duration of response (DOR) was 30 months, with an OS of 11.3 months; in patients with a combined positive score of 10 or higher, the objective response rate (ORR) was $47 \%$ and median OS was 18.5 months. This was similar to that of patients with lymph node-only disease. In the $42 \%$ of patients with an ECOG performance status of 2 and in the $38 \%$ of patients who were older than 75 years, the response rates and overall outcomes were similar. ${ }^{7}$

Patients treated in the second-line setting have a poor prognosis, with a median OS of less than 6 months. Between 2016 and 2017, atezolizumab, nivolumab, avelumab, durvalumab, and pembrolizumab were approved by the FDA as second-line treatment for advanced or metastatic UC following the failure of platinum-based systemic treatment. This approval came after convincing data from the phase 2 IMvigor 210, ${ }^{8}$ phase 2 CheckMate 275 (NCT02387996), ${ }^{9}$ phase 1 JAVELIN solid tumor (NCT01772004), ${ }^{10}$ phase $1 / 2$ STUDY 1108 trial (NCT01693562), ${ }^{11}$ and phase 3 KEYNOTE-045 trials (NCT02256436). ${ }^{12}$ Table 2 describes the outcomes of these trials in second-line post-platinum therapy.

\subsection{ICls in combination with chemotherapy in mUC}

Cytotoxic therapy can promote antitumor immunity by modifying the tumor-suppressive microenvironment composition.

TABLE 1. First-line ICI Monotherapy in mUC

\begin{tabular}{|c|c|c|}
\hline & KEYNOTE52/pembrolizumab & $\begin{array}{l}\text { IMvigor210 (cohort 1)/ } \\
\text { atezolizumab }\end{array}$ \\
\hline \multicolumn{3}{|c|}{ Single-arm phase 2 trial, cisplatin ineligible, treatment naïve in the metastatic setting } \\
\hline $\mathbf{N}$ & 370 & 119 \\
\hline ORR & $29 \%$ & $23 \%$ \\
\hline - CR & $9 \%$ & $9 \%$ \\
\hline - PR & $20 \%$ & $14 \%$ \\
\hline \multirow[t]{3}{*}{ - PD-L1 status } & CPS $\geq 10: 47 \%$ & ICO: $8 \%$ \\
\hline & CPS < 10: $20 \%$ & IC1: $\quad 10 \%$ \\
\hline & & IC2/3: 9\% \\
\hline - Lymph node only & $49 \%$ & $32 \%$ \\
\hline Median OS & $11.3 \mathrm{mo}$ & $15.9 \mathrm{mo}$ \\
\hline \multirow[t]{2}{*}{ - PD-L1 status } & $\mathrm{CPS} \geq 10: 18.5 \mathrm{mo}$ & IC0/1: $19.1 \mathrm{mo}$ \\
\hline & CPS < 10: $9.7 \mathrm{mo}$ & IC2/3: $12.3 \mathrm{mo}$ \\
\hline - Lymph node only & $27.0 \mathrm{mo}$ & N/A \\
\hline Median DOR & $\begin{array}{l}30.1 \mathrm{mo} \\
(95 \% \mathrm{Cl}, 18.1-\mathrm{NR})\end{array}$ & N/A \\
\hline Median PFS & $2.2 \mathrm{mo}$ & $2.7 \mathrm{mo}$ \\
\hline TRAEs & $67.3 \%$ & $66 \%$ \\
\hline - Grade 3/4 TRAEs & $20.8 \%$ & $16 \%$ \\
\hline
\end{tabular}

CPS, combined positive score; CR, complete response; DOR, duration of response; IC, tumor-infiltrating immune cells; $\mathrm{ICl}$, immune checkpoint inhibitor; mo, months; mUC, metastatic urothelial carcinoma; N/A, not applicable; NR, not reached; ORR, objective response rate; OS, overall survival; PFS, progression-free survival; PR, partial response; TRAE, treatment-related adverse event.

The release of "danger" molecules from tumor cells, such as calreticulin, polarizes dendritic cells toward a proinflammatory phenotype, thus increasing priming toward T helper 1 cells and away from regulatory T cells. Multiple lines of evidence suggest that immunotherapies combined with chemotherapy may deliver a greater potency than ICI monotherapy. ${ }^{13,14}$

To test this hypothesis, the phase 3 KEYNOTE-361 (NCT02853305) and phase 3 IMvigor130 (NCT02807636) trials randomized first-line patients with mUC who were cisplatin eligible to pembrolizumab or atezolizumab monotherapy; ICI in combination with chemotherapy; or chemotherapy alone. The primary end point for both was progression-free survival (PFS). In both studies, the ICI-only arm in patients with low PDL1 tumors was ended early after an FDA alert in May 2018 warned of decreased survival in this group. In both trials, the primary end point was not reached. In KEYNOTE-361, the PFS in the pembrolizumab/chemotherapy combination arm was 8.3 months compared with 7.1 months in the chemotherapy-only arm (HR, 0.78; 95\% CI, 0.65-0.93; P = $.0033)$; OS was 17.0 vs 14.3 months, respectively (HR, 0.86; $95 \%$ CI, 0.72-1.02; $P=.0407) .{ }^{15}$ In the IMvigor 130 trial at a median follow-up of 11.8 months, median PFS was 8.2 months in the combination arm vs 6.3 months in the chemotherapyonly arm (HR, 0.82; 95\% CI, 0.70-0.96; $P=.007)$ and median OS was 16.0 vs 13.4 months, respectively (Table 3 ). ${ }^{16}$ The IMvigor130 and KEYNOTE-361 trials currently support chemotherapy as firstline therapy for platinum-eligible patients. When combined with chemotherapy, ICI does not appear to confer any additional benefit in ORR, PFS, or OS. 
Sequential therapy with chemotherapy then maintenance ICI is the current standard of care (SOC) based on the phase 3 JAVELIN 100 trial (NCT02603432), which demonstrated improvement in OS for avelumab vs placebo post chemotherapy in patients with stable disease or better response to first-line chemotherapy. ${ }^{17}$

The use of lower doses of chemotherapy to stimulate an immune response is also being investigated in a phase 2 trial (NCT02581982) in the second-line setting with low-dose paclitaxel and pembrolizumab. Data presented at the 2021 American Society of Clinical Oncology Genitourinary Annual Meeting (ASCO GU) reported a response rate of $33 \%$. The 6 -month PFS was $46.8 \%$ (95\% CI, $27 \%-64.2 \%)$ and median OS was 11.7 months $(95 \%$ CI, 8.7 to not reached). This preliminary result illustrates that the addition of a low dose of paclitaxel to pembrolizumab results in a favorable response and is tolerable. However, this should be confirmed in a phase 3 setting. ${ }^{18}$ doublets (ie, an anti-PD-1/PD-L1 agent plus an anti-CTLA-4 agent) vs chemotherapy as first-line treatment for mUC in both platinum-eligible and -ineligible patients. Tremelimumab is a fully humanized immunoglobulin G2 monoclonal antibody against CTLA-4. The DANUBE trial (NCT 02516241) evaluated the efficacy in mUC of durvalumab vs durvalumab/tremelimumab, followed by durvalumab maintenance vs chemotherapy. The primary end points of the trial were PFS and OS of the durvalumab/tremelimumab combination vs SOC, stratifying for PD-L1 status. The trial did not meet its coprimary end points. ${ }^{22}$ In the high-PD-L1 population, OS was 14.4 months with durvalumab alone vs 12.1 months in the chemotherapy group (HR, 0.89; 95\% CI, 0.71$1.11 ; P=.3)$. Overall OS was 15.1 months with durvalumab/tremelimumab vs 12.1 months with chemotherapy (HR, 0.85 ; 95\% CI, 0.72-1.02; $P=.075$ ) in the intent-to-treat population.

\section{Due to the different and lower toxicity profiles of these [immunotherapy oncology] agents, their approvals in the first-line platinum-ineligible and second-line metastatic settings expanded our ability to treat a broader population of patients with metastatic disease.}

\subsection{Dual immunotherapy in $\mathbf{m U C}$}

Combination therapy with both antiPD-1/PD-L1 and anti-CTLA-4 agents has proven synergistic effects, and ipilimumab (Yervoy)/nivolumab has been approved in the treatment of melanoma, renal cell carcinoma, and microsatellite instability-high colon cancer. However, the combination has higher toxicity than monotherapy. ${ }^{19-21}$

There are three phase 3 trials studying immunotherapy oncology (IO)
Currently accruing trials include NILE (NCT03682068), which is investigating the combinations of durvalumab/tremelimumab/chemotherapy vs durvalumab/ chemotherapy vs chemotherapy alone in the first-line setting; and CheckMate 901 (NCT03036098), which is studying nivolumab with either ipilimumab or chemotherapy vs chemotherapy alone, with primary end points of PFS and OS. Both trials are accruing patients who are cisplatin eligible and ineligible.

\subsection{ICls in combination with antibody-drug conjugates}

$\mathrm{EV}$, an antibody-drug conjugate (ADC) that targets Nectin-4, was approved in December 2019 based on the results of EV-201, a phase 2 study in patients with prior chemotherapy and ICI therapy. ${ }^{23}$ Approval was based on cohort 1, which included 125 patients who had progressed on both ICI and chemotherapy, with a confirmed response rate of $44 \%$ reported. The disease progression rate was $18 \%$, translating to an $82 \%$ clinical benefit rate (CBR) in all subgroups. OS was 12.4 months (95\% CI, 9.46-15.57). At ASCO GU 2021, the results of cohort 2 (cisplatin-ineligible patients with prior IO therapy) were reported; their ORR was $52 \%$, including $20 \%$ who had a complete response (CR). Median PFS and median OS were 5.8 months and 14.7 months, respectively. ${ }^{24}$

EV-301 (NCT03474107), a randomized phase 3 trial evaluating EV vs chemotherapy alone in patients with previously treated mUC, demonstrated median OS of 12.9 months with EV vs 9.0 months with chemotherapy (HR, 0.70; $95 \%$ CI, 0.560.89 ; 1 -sided $P=.001$ ) after a median follow-up of 11.1 months. The incidence of treatment-related adverse events (TRAEs) was $93.9 \%$ vs $91.8 \%$, and the incidence of grade 3 or higher TRAEs was $51.4 \%$ vs $49.8 \%$, in the EV and chemotherapy groups, respectively. ${ }^{25}$

In the phase 1/2 EV-103 trial which combined EV with pembrolizumab in cisplatin-ineligible patients in the firstline setting, the combination had an ORR of $73 \%$ and an additional $20 \%$ of patients experienced stable disease (disease control rate $93 \%$ ). The results did not appear to be dependent on PD-L1 expression. ${ }^{26}$ This rate of high response to EV both as monotherapy and in combination with pembrolizumab is promising, and it has resulted in several ongoing trials including EV-302 (NCT04223856), an open-label, randomized phase 3 trial studying EV in combination with pembrolizumab vs chemotherapy alone in 
TABLE 2. ICls in Platinum-Refractory mUC

\begin{tabular}{|c|c|c|c|c|c|}
\hline Agent & Atezolizumab & Pembrolizumab & Nivolumab & Avelumab & Durvalumab \\
\hline Trial & $\begin{array}{l}\text { IMvigor } 210 \text { (cohort 2): } \\
\text { phase 2, single arm }\end{array}$ & $\begin{array}{c}\text { KEYNOTE-045: } \\
\text { phase 3, randomized }\end{array}$ & $\begin{array}{l}\text { CheckMate 275: } \\
\text { phase 2, single arm }\end{array}$ & $\begin{array}{l}\text { JAVELIN Solid Tumor: } \\
\text { phase } 1 \mathrm{~b}\end{array}$ & $\begin{array}{l}\text { Study 1108: } \\
\text { phase } 1 / 2\end{array}$ \\
\hline $\mathbf{N}$ & 310 & 270 & 265 & 249 & 191 \\
\hline ORR & $15 \%$ & $21 \%$ & $20 \%$ & $16 \%$ & $18 \%$ \\
\hline Median DOR & $24.8 \mathrm{mo}$ & $29.7 \mathrm{mo}$ & $17.7 \mathrm{mo}$ & $20.5 \mathrm{mo}$ & N/A \\
\hline Median OS & $7.9 \mathrm{mo}$ & $10.3 \mathrm{mo}$ & $8.7 \mathrm{mo}$ & $7.0 \mathrm{mo}$ & $18.2 \mathrm{mo}$ \\
\hline Median PFS & $2.1 \mathrm{mo}$ & $2.1 \mathrm{mo}$ & $2.0 \mathrm{mo}$ & $1.6 \mathrm{mo}$ & $1.5 \mathrm{mo}$ \\
\hline Grade $3 / 4$ TRAEs & $16 \%$ & $15 \%$ & $18 \%$ & $12 \%$ & $7 \%$ \\
\hline
\end{tabular}

DOR, duration of response; ICl, immune checkpoint inhibitor; mo, months; mUC, metastatic urothelial carcinoma; N/A, not applicable;

ORR, objective response rate; OS, overall survival; PFS, progression-free survival; TRAE, treatment-related adverse event.

previously untreated locally advanced or metastatic UC.

Sacituzumab govitecan (Trodelvy) is another ADC targeting trophoblast cell surface antigen-2 (Trop-2) linked to SN38, a topoisomerase inhibitor, that has shown promising activity in the thirdline setting. Trop- 2 is a transmembrane calcium signal transducer that is highly expressed in many human epithelial malignancies. ${ }^{27}$ It regulates cancer growth, invasion, and metastasis by multiple signaling pathways. ${ }^{28}$ Recent data from cohort 1 of the phase 2 TROPHY-U-01 trial (NCT03547973) reported an ORR of $27 \%$ with sacituzumab govitecan in patients with mUC who were previously treated with chemotherapy and PD-1/ PD-L1 therapy. CR was reported in 5\% of patients, and $22 \%$ achieved partial response. ${ }^{29,30}$ Given the promising data from cohort 1 , the response rate to and safety of sacituzumab govitecan combined with pembrolizumab will be assessed in cohort 3 patients who progressed after prior platinum-based therapies but have not received immunotherapy.

Additionally, the FDA has recently granted accelerated approval to sacituzumab govitecan for locally advanced or metastatic UC previously treated with platinum-based chemotherapy and immunotherapy. ${ }^{31} \mathrm{~A}$ phase 3 trial, TROPiCS-04 (NCT04527991), has also been initiated to compare sacituzumab govitecan with physician's choice (singleagent taxane or vinflunine) in patients with metastatic or locally advanced unresectable UC who were treated with prior chemotherapy and ICI therapy. ${ }^{32}$

\subsection{ICls in combination with targeted therapy in mUC}

Combinatorial regimens of ICI and targeted therapy can result in synergistic effects (by targeting complementary pathways), improve outcomes of immunotherapy (by sensitizing tumor cells and improving effector cells), and overcome resistance to checkpoint therapy. Some targeted therapies may directly modulate the immune response by attenuating the activities of specific immune cell populations that restrain cytotoxic T lymphocytes. ${ }^{33,34}$

Erdafitinib, an FGFR inhibitor, received accelerated approval from the FDA in 2019 as the first targeted drug for patients with either locally advanced mUC or mUC harboring the FGFR2/3 mutation or gene fusion (found in approximately $20 \%$ of patients with mUC). A recent study showed that inhibiting erdafitinib led to upregulation of BST2, a gene associated with activated interferon signaling and the immune system. ${ }^{35}$ Patients with FGFR mutations or fusions may have lower sensitivity to ICI.

Table 3 describes the current trials with erdafitinib and ICI combinations. An ongoing phase $1 \mathrm{~b} / 2$ trial (NCT03473743) is studying the safety, tolerability, and efficacy of the combination of erdafitinib with cetrelimab, a PD-1 antibody, in cisplatin-ineligible patients with locally advanced or mUC harboring FGFR gene alterations.

The FORT-2 study (NCT03473756) is a phase $1 \mathrm{~b} / 2$ trial studying the combination of atezolizumab with rogaratinib, an FGFR inhibitor, as first-line treatment in cisplatin-ineligible patients harboring the FGFR alteration, with primary end points of toxicity and PFS.

FIERCE-22 (NCT03123055) is a phase $1 \mathrm{~b} / 2$ single-arm trial evaluating the combination of pembrolizumab with vofatamab, an FGFR3 inhibitor, in patients with platinum-refractory mUC. The combination was active in patients with both wild-type and mutated FGFR3, with an ORR of $29.6 \%{ }^{36}$

BISCAY was a novel biomarker-directed platform that sought to evaluate ICI in combination with targeted therapies in patients with platinum-refractory mUC. Combinations of durvalumab with AZD4547 (an FGFR inhibitor), olaparib (Lynparza; a PARP inhibitor), and vistusertib (a TORC inhibitor) were evaluated. Unfortunately, the overall response to durvalumab was not amplified by adding any of these targeted agents. ${ }^{37}$

The tryptophan catalytic enzyme IDO1 
is an important immune regulatory protein; it can drive cancer immunosuppression by stimulating regulatory $T$ cells and suppressing CD8 T effector cells, and it can be upregulated by anti-PD-1 agents. Linrodostat mesylate, an oral IDO1 inhibitor, has been evaluated in combination with nivolumab in the phase $1 / 2$ advBC trial (NCT02658890) for patients with mUC who were not previously treated by ICIs, following 1 or more prior lines of therapy. Results included an ORR of $37 \%$ and a disease control rate of $56 \% .^{38}$

MARIO-275 (NCT03980041) is a randomized phase 2 trial evaluating the safety and efficacy of the PI3K-gamma inhibitor eganelisib combined with nivolumab in patients with $\mathrm{mUC}$ who have progressed

\section{TABLE 3. Randomized Phase 3 Immuno-Oncology Combination Therapy in mUC}

\begin{tabular}{|c|c|c|c|c|c|}
\hline \multicolumn{6}{|c|}{ Combination with chemotherapy } \\
\hline & \multicolumn{2}{|l|}{ IMvigor 130} & \multicolumn{3}{|c|}{ KEYNOTE-361 } \\
\hline Arms & \multicolumn{2}{|l|}{$\begin{array}{l}\text { - Atezolizumab } \\
\text { - Chemotherapy } \\
\text { - Atezolizumab/chemotherapy }\end{array}$} & \multicolumn{3}{|c|}{$\begin{array}{l}\text { - Pembrolizumab } \\
\text { - Chemotherapy } \\
\text { - Pembrolizumab/chemotherapy }\end{array}$} \\
\hline PFS & \multicolumn{2}{|l|}{$\begin{array}{l}\text { - Chemotherapy: } 6.3 \text { mo } \\
\text { - Atezolizumab/chemotherapy: } 8.2 \text { mo } \\
\text { - HR, } 0.82(95 \% \mathrm{Cl}, 0.70-0.96) \\
\text { - } \mathrm{P}=.007\end{array}$} & \multicolumn{3}{|c|}{$\begin{array}{l}\text { - Chemotherapy: } 7.1 \text { mo } \\
\text { - Pembrolizumab/chemotherapy: } 8.3 \text { mo } \\
\text { - P = .0033 }\end{array}$} \\
\hline OS & \multicolumn{2}{|l|}{$\begin{array}{l}\text { - Chemotherapy: } 13.4 \text { mo } \\
\text { - Atezolizumab/chemotherapy: } 16.0 \text { mo } \\
\text { - HR, } 0.83(95 \% \mathrm{Cl}, 0.69-1.00) \\
\text { - P }=.027\end{array}$} & \multicolumn{3}{|c|}{$\begin{array}{l}\text { - Chemotherapy: } 14.3 \text { mo } \\
\text { - Pembrolizumab/chemotherapy: } 17.0 \text { mo } \\
\text { - P =.0407 }\end{array}$} \\
\hline \multicolumn{6}{|c|}{ Dual immunotherapy } \\
\hline \multicolumn{2}{|r|}{ NILE } & \multicolumn{2}{|l|}{ CheckMate 901} & \multicolumn{2}{|r|}{ DANUBE } \\
\hline Arms & $\begin{array}{l}\text { - Durvalumab/chemotherapy } \\
\text { - Durvalumab/tremelimumab/ } \\
\text { chemotherapy } \\
\text { - Chemotherapy }\end{array}$ & \multicolumn{2}{|c|}{$\begin{array}{l}\text { - Nivolumab/ipilimumab } \\
\text { - Nivolumab/chemotherapy } \\
\text { - Chemotherapy }\end{array}$} & \multicolumn{2}{|c|}{$\begin{array}{l}\text { - Durvalumab } \\
\text { - Durvalumab/tremelimumab } \\
\text { - Chemotherapy }\end{array}$} \\
\hline $\mathbf{O S}$ & N/A & $\mathrm{N} / \mathrm{A}$ & & \multicolumn{2}{|c|}{$\begin{array}{l}\text { - Durvalumab vs chemo: } 14.4 \text { mo vs } 12.1 \mathrm{mo} \text {; } \\
\text { HR, } 0.89 \text { (95\% Cl, 0.71-1.11; P = .3039) } \\
\text { - Durvalumab/tremelimumab vs chemother- } \\
\text { apy: } 15.1 \mathrm{mo} \text { vs } 12.1 \mathrm{mo} ; \mathrm{HR}, 0.85(95 \% \mathrm{Cl} \text {, } \\
\text { 0.72-1.02; P = .0751) }\end{array}$} \\
\hline \multicolumn{6}{|c|}{ Combination with antibody-drug conjugates } \\
\hline & \multicolumn{2}{|l|}{ EV-103 } & \multicolumn{3}{|c|}{ TROPHY-U-01 Cohort 3} \\
\hline Trial & \multicolumn{2}{|c|}{ Phase $1 \mathrm{~b} / 2$ study in cisplatin-ineligible, first-line $\mathrm{mUC}$} & \multicolumn{3}{|c|}{$\begin{array}{l}\text { Single-arm, phase } 2 \text { study in progressive disease after prior } \\
\text { platinum-based therapies, but ICI naïve. }\end{array}$} \\
\hline Arms & \multicolumn{2}{|l|}{ - Enfortumab vedotin/pembrolizumab } & \multicolumn{3}{|c|}{ - Sacituzumab govitecan/pembrolizumab } \\
\hline Results & \multicolumn{2}{|l|}{$\begin{array}{l}\text { - ORR: } 73.3 \%(95 \% \text { Cl, 58.1\%-85.4\%) } \\
\text { - CR: } 15.6 \% \\
\text { - PR: } 57.8 \% \\
\text { - Median PFS: } 12.3 \text { mo } \\
\text { - 12-mo OS: } 81.6 \%\end{array}$} & \multicolumn{3}{|c|}{ - Ongoing } \\
\hline \multicolumn{6}{|c|}{ Combination with FGFR inhibitors } \\
\hline \multicolumn{2}{|r|}{ BISCAY } & FORT-2 & \multicolumn{2}{|r|}{ FIERCE-22 } & NCT03473743 \\
\hline Trial & $\begin{array}{l}\text { Durvalumab combination with FGFR } \\
\text { inhibitor AZD4547, or PARP inhibitor } \\
\text { olaparib, or TORC inhibitor vistusertib }\end{array}$ & $\begin{array}{l}\text { Phase } 1 \mathrm{~b} / 2 \text { trial studying } \\
\text { FGFR inhibitor rogaratinib } \\
\text { with atezolizumab }\end{array}$ & & $\begin{array}{l}\text { Phase } 1 \mathrm{~b} / 2 \text {, single-arm } \\
\text { trial, evaluating FGFR3 } \\
\text { inhibitor vofatamab } \\
\text { with pembrolizumab }\end{array}$ & $\begin{array}{l}\text { Phase } 1 \mathrm{~b} / 2 \text { trial to investi- } \\
\text { gate erdafitinib with PD- } 1 \\
\text { antibody cetrelimab }\end{array}$ \\
\hline Results & - No difference in ORR & - Ongoing & & Terminated & - Ongoing \\
\hline
\end{tabular}

DOR, duration of response; ICls, immune checkpoint inhibitor; mo, months; mUC, metastatic urothelial carcinoma; N/A, not applicable; ORR, overall response rate; OS, overall survival; PFS, progression-free survival; PR, partial response. 

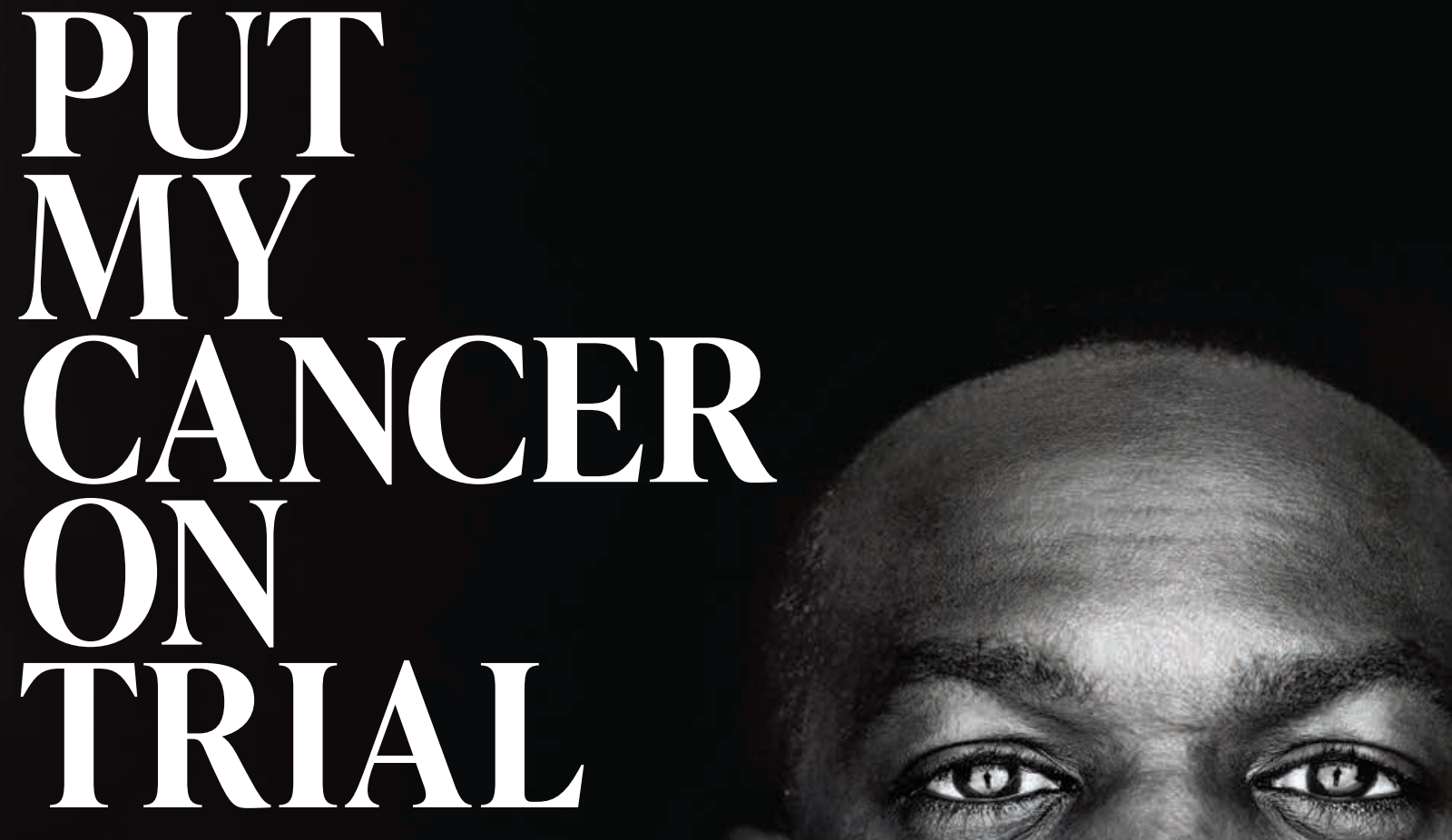

Shawn, 45

Diagnosed with colorectal cancer

\section{Cancer doesn't discriminate. So why do our clinical trials?}

African Americans make up 13\% of the US population but only $3 \%$ of participants in clinical trials for cancer.*

Clinical trials are a lifeline, and often the only way someone with advanced cancer can survive. The time is now to end racial disparities in cancer clinical trials.

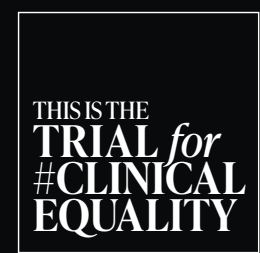

Demand change. Take action. ClinicalEquality.com

\section{FCBHEALTH}

NETWORK

*Percentages of patients calculated from trials supporting FDA drug approvals between July 2008 and June 2018 that documented the inclusion of White, Asian, Black, and Hispanic patients. 
on a platinum-based chemotherapy regimen but who are immunotherapy naïve. Eligible patients were randomized 2:1 to receive eganelisib in combination with nivolumab or placebo with nivolumab, stratified by the level of baseline circulating monocytic myeloid-derived suppressor cells (MDSCs). One of the mechanisms of PI3K-gamma is to maintain the immunosuppressive role of macrophages and MDSCs. Preliminary results showed that the primary end point, ORR, was higher in the eganelisib/nivolumab arm compared with the placebo/nivolumab arm in both the low monocytic MDSC subgroup (38.5\% vs $23.1 \%$, respectively) and the PD-L1 negative subgroup (26.1\% vs $14.3 \%$, respectively). TRAEs were more common in the eganelisib/nivolumab arm even after dose reduction. ${ }^{39}$

\section{MIBC}

The standard treatment for patients with

\section{TABLE 4. Neoadjuvant ICI Combination Therapy in MIBC}

\begin{tabular}{|c|c|c|c|c|c|c|}
\hline \multicolumn{7}{|c|}{ Dual immunotherapy } \\
\hline \multicolumn{3}{|c|}{ NABUCCO } & \multicolumn{2}{|l|}{ NCT02812420 } & \multicolumn{2}{|c|}{ DUTRENEO* } \\
\hline Trial & \multicolumn{2}{|l|}{ Phase 1} & \multicolumn{2}{|l|}{ Phase 1} & \multicolumn{2}{|l|}{ Phase 2 , randomized } \\
\hline Arms & \multicolumn{2}{|l|}{ - Nivolumab/ipilimumab } & \multicolumn{2}{|l|}{$\begin{array}{l}\text { - Durvalumab/ } \\
\text { tremelimumab }\end{array}$} & \multicolumn{2}{|c|}{$\begin{array}{l}\text { - "Cold" tx chemo } \\
\text { - "Hot" tx chemo } \\
\text { - "Hot" tx durvalumab/tremelimumab }\end{array}$} \\
\hline Sample & \multicolumn{2}{|c|}{$\begin{array}{l}\text { - } 24 \text { patients refused or ineligi- } \\
\text { ble for cisplatin } \\
\text { - cT1-4N1-3 }\end{array}$} & \multicolumn{2}{|c|}{$\begin{array}{l}\text { - } 28 \text { cisplatin ineligible } \\
\text { and high-risk patients, } \\
\text { - cT1-T4 N0 }\end{array}$} & \multicolumn{2}{|c|}{$\begin{array}{l}\text { - } 61 \text { cisplatin-eligible patients } \\
\text { - cT2-4/or N+ }\end{array}$} \\
\hline pTONO rate & \multicolumn{2}{|l|}{ - $46 \%$} & \multicolumn{2}{|l|}{ - 37.5\% } & \multicolumn{2}{|c|}{$\begin{array}{l}\text { - "Cold" chemo: } 68.8 \% \\
\text { - "Hot" chemo: } 36.4 \% \\
\text { - "Hot" durvalumab/tremelimumab: } 34.8 \%\end{array}$} \\
\hline$\leq$ pT1No rate & \multicolumn{2}{|l|}{ - $61 \%$} & \multicolumn{2}{|l|}{ - $58 \%$} & \multicolumn{2}{|c|}{$\begin{array}{l}\text { - "Cold" chemo: } 75.0 \% \\
\text { - "Hot" chemo: } 59.1 \% \\
\text { - "Hot" durvalumab/tremelimumab: } 56.5 \%\end{array}$} \\
\hline Biomarkers & \multicolumn{2}{|c|}{$\begin{array}{l}\text { - CR rate: } 73 \% \text { if } P D-L 1 \text { positive } \\
\text { - } C R \text { rate: } 33 \% \text { if } P D-L 1 \text { nega- } \\
\text { tive }(P=.15)\end{array}$} & \multicolumn{2}{|c|}{$\begin{array}{l}\text { - Response rate not relat- } \\
\text { ed to PD-L1 expression, } \\
\text { TMB, or DDR }\end{array}$} & \multicolumn{2}{|c|}{$\begin{array}{l}\text { - Higher PD-L1 expression predicted higher pCR rate } \\
\text { only when treated with combined } \mathrm{ICI} \text {, not when treated } \\
\text { with chemotherapy }\end{array}$} \\
\hline \multicolumn{7}{|c|}{ Combination with chemotherapy } \\
\hline \multicolumn{3}{|c|}{ GU14-188-C1 } & \multicolumn{2}{|l|}{ GU14-188-C2 } & BLASST-1 & SAKK06/17 \\
\hline Trial & Phase $1 \mathrm{~b} / 2$ & \multicolumn{2}{|c|}{ Phase 1b/2 } & \multicolumn{2}{|c|}{ Phase 2} & Phase 2 \\
\hline Arms & $\begin{array}{l}\text { - Gemcitabine/cisplatin/ } \\
\text { pembrolizumab }\end{array}$ & \multicolumn{2}{|c|}{$\begin{array}{l}\text { - Gemcitabine/ } \\
\text { pembrolizumab }\end{array}$} & \multicolumn{2}{|c|}{$\begin{array}{l}\text { - Gemcitabine/cisplatin/ } \\
\text { nivolumab }\end{array}$} & $\begin{array}{l}\text { - Gemcitabine/cisplatin/ } \\
\text { durvalumab }\end{array}$ \\
\hline Samples & $\begin{array}{l}\text { - } 43 \text { cisplatin-eligible pa- } \\
\text { tients with cT2-4aNoM0 }\end{array}$ & \multicolumn{2}{|c|}{$\begin{array}{l}\text { - } 37 \text { cisplatin-ineligible } \\
\text { patients with cT2-4aN0M0 }\end{array}$} & \multicolumn{2}{|c|}{$\begin{array}{l}\text { - } 41 \text { cisplatin-eligible } \\
\text { patients with cT2-T4a, } \mathrm{N} \leq \text {, M0 }\end{array}$} & $\begin{array}{l}\text { - 30/61 cisplatin-eligible } \\
\text { patients with cT2-T4a cNo-1 }\end{array}$ \\
\hline pTONO rate & - $44.4 \%$ & \multicolumn{2}{|c|}{ - $45.2 \%$} & .48 & $7 \%$ & - $30.0 \%$ \\
\hline$\leq \mathrm{pT} 1 \mathrm{NO}$ rate & - $61.1 \%$ & - 51. & & -65 & & - 50.0\% \\
\hline Biomarkers & $\begin{array}{l}\text { - Response rate not } \\
\text { related to PD-L1 score }\end{array}$ & $\begin{array}{l}\text { - Re } \\
\text { ed to }\end{array}$ & $\begin{array}{l}\text { ponse rate not relat- } \\
\text { PD-L1 score }\end{array}$ & $\begin{array}{l}-\mathrm{Re} \\
\mathrm{PD}-\end{array}$ & $\begin{array}{l}\text { sponse rate not related to } \\
-1 \text { score }\end{array}$ & - N/A \\
\hline & & & Combination with $t_{c}$ & $\operatorname{arget} t$ & lerapy & \\
\hline & NEODL & RVARI & & & ABATE & trial \\
\hline Trial & Phase 2 & & & Phase & & \\
\hline Arms & - Durvalumab/olaparib & & & - Cab & Ozantinib/atezolizumab & \\
\hline Samples & - cT2-T4a & & & - cT2 & T4a & \\
\hline pTONO rate & - $50 \%$ & & & - N/A & & \\
\hline$\leq \mathrm{pT} 1 \mathrm{No}$ rate & - N/A & & & - N/A & & \\
\hline
\end{tabular}

*DUTRENEO trial "hot" and "cold" tumors determined by proinflammatory interferon-gamma signature and tumor inflammation signature. DDR, DNA damage response; MIBC, muscle-invasive bladder cancer, N/A, not applicable; pCR, pathologic complete response;

TMB, tumor mutation burden; tx, treatment. 
MIBC is neoadjuvant chemotherapy followed by radical cystectomy with a recent meta-analysis showing an absolute OS benefit of $5 \%$. Local recurrence was $30 \%$ to $54 \%$ with a $50 \%$ reduction in the risk of distant disease with neoadjuvant therapy followed by radical cystectomy. ${ }^{40,41}$ Pathological response is a surrogate for OS. ${ }^{42-44}$ However, as a result of cisplatin ineligibility $(50 \%)$ or refusal to receive chemotherapy $(30 \%)$, only about $20 \%$ of patients with MIBC are treated with cisplatin-based neoadjuvant chemotherapy. ${ }^{40}$

Due to the success of IO therapy in the metastatic setting, multiple trials of ICIs in the neoadjuvant setting for MIBC are currently accruing. The agents include ICI monotherapy and combinations of IO/ chemotherapy and $\mathrm{IO} /$ targeted therapies.

Both the phase 2 PURE-01 (NCT02736266) and phase 2 ABACUS trials (NCT02662309) evaluating pembrolizumab and atezolizumab reported favorable pathological response rates in the neoadjuvant setting. Pathological CR (pCR) in the PURE-01 trial was $42 \%$ and an additional $12 \%$ patients were either $\mathrm{pTa}$, Tis or $\mathrm{pT} 1$. PD-L1 expression was associated with higher $\mathrm{pCR}$ response. ${ }^{45}$ In the ABACUS trial, the pCR rate was $31 \%$, with no significant correlation with PD-L1 expression. ${ }^{46}$ The pCR rates from the PURE-01 and ABACUS trials are similar to historic rates reported with neoadjuvant chemotherapy (38\% with MVAC) ${ }^{42}$ and with fewer toxicities. Given these promising phase 2 data, ICI combination therapy is being investigated in the neoadjuvant setting.

\subsection{Neoadjuvant dual immunotherapy with MIBC}

NABUCCO (NCT03387761) is a single-arm phase $1 \mathrm{~b}$ trial that studied the feasibility of preoperative ipilimumab plus nivolumab in MIBC, with 24 enrolled patients. Eleven patients $(46 \%)$ achieved $\mathrm{pCR}$ ( $\mathrm{pTON} 0)$ and $58 \%$ of patients were downstaged to noninvasive disease. The pCR rate was $73 \%$ in the PD-L1-positive group and $33 \%$ in the PD-L1-negative group $(P=.15) .{ }^{47}$ Currently, a nonrandomized phase 2 study designed to evaluate neoadjuvant nivolumab vs nivolumab and ipilimumab in patients with MIBC who are ineligible for cisplatin-based chemotherapy (NCT03520491) is underway and still recruiting (Table 4).

\section{In the muscle invasive setting, the response rates to anti-PD-1/L1... appear to achieve pCR and pathological non- muscle invasive rates that compare favorably with historical controls.}

The results of a pilot trial of the combination of durvalumab and tremelimumab as neoadjuvant therapy (NCT02812420) have been reported for cisplatin-ineligible patients with high-risk disease, defined as bulky tumors, variant histology, lymphovascular invasion, hydronephrosis, or high-grade upper tract disease. ${ }^{48,49} \mathrm{The}$ primary end point was safety, and $21.4 \%$ of patients had grade $\geq 3$ immunotherapy-related toxicity; $37.5 \%$ of patients achieved pCR and $58.0 \%$ of patients downstaged to pT1 or less. ${ }^{48}$

The phase 2 DUTRENEO trial (NCT03472274) sought to evaluate tumor proinflammatory interferon-gamma tumor inflammation signature (TIS) to predict which patients might benefit from dual IO therapy (durvalumab/tremelimumab) vs chemotherapy as neoadjuvant therapy. ${ }^{50}$ Patients with cT2-4/or who were $\mathrm{N}+$ stage and cisplatin eligible were classified as having "hot" or "cold" tumors according to TIS score determined by Nanostring technology. ${ }^{51}$ Patients with "hot" tumors were randomized to a cisplatin-based chemotherapy group or a combined chemotherapy/ICI group; patients with "cold" tumors were treated with cisplatin-based chemotherapy. The pCR rate was $68.8 \%$ in the "cold" chemotherapy group, $36.4 \%$ in the "hot" chemotherapy group, and $34.8 \%$ in the "hot" combined IO group. Tumors with higher PD-L1 expression predicted higher pCR rate only when treated with combined IO, not with chemotherapy..$^{50}$ The AE profile of combined IO therapy seemed to be favorable compared with that of chemotherapy. However, the role of TIS score in immunotherapy selection is still unclear. Further study is required to validate this biomarker.

\subsection{Neoadjuvant ICI/ chemotherapy combination treatments in MIBC}

Numerous clinical trials with ICI/chemotherapy combinations in the neoadjuvant setting are underway for MIBC (Table 4). The GU14-188 trial (NCT02365766) is a phase $1 \mathrm{~b} / 2$ study evaluating neoadjuvant pembrolizumab with gemcitabine/cisplatin (GC) in cisplatin-eligible) and gemcitabine alone in cisplatin-ineligible patients with T2-4aN0M0 bladder cancer. The primary end points were toxicity and pathologic non-muscle-invasive response rate (PaIR) (ie, ypT0, Tis, Ta,T1, and N0). ${ }^{52}$ A total of 42 patients were enrolled in the cisplatin-eligible cohort. With pembrolizumab/GC, the PaIR was $61 \%$ and the pCR rate was $44 \%$. Neither correlated with the baseline PD-L1 score. At a median follow-up of 34.2 months, the estimated 36-month recurrence-free survival was $63 \%$ and OS was $82 \%$. Cohort 2 of the GU14-188 trial was designed to study the tolerability and efficacy of neoadjuvant gemcitabine with pembrolizumab in cisplatin-ineligible patients with cT24aNOM0 MIBC, with PaIR as the primary end point. The interim results were similar to those of cohort 1 ; PaIR was $51.6 \%$ and the pCR rate was $45.2 \%$, and the response rate was not related to the baseline PDL1 score..$^{53}$ Given that the current SOC is dose-dense MVAC, with a pCR rate of $38 \%,{ }^{42}$ the results of GU14-188 suggest 
that the pembrolizumab/GC combination is feasible with manageable toxicity.

Another phase 2 trial (NCT02690558) recently reported a $36 \%$ pCR rate and PaIR of $56 \%$ with neoadjuvant pembrolizumab plus gemcitabine and a split dose of cisplatin in patients with clinical T24a N0/X M0 urothelial bladder cancer who were eligible for cisplatin. ${ }^{54}$

The BLASST-1 trial (NCT03294304) was a phase 2 study of neoadjuvant nivolumab with GC in patients with MIBC prior to radical cystectomy (RC). The primary end point was non-muscle invasive disease $(\mathrm{y} p T \leq 1)$. Secondary end points were the safety of GC plus nivolumab and PFS at 2 years. A total of 41 patients were enrolled and $65.8 \%$ responded, including patients with $\mathrm{cN} 1$ disease. The $\mathrm{pCR}$ rate was $49 \%$ and response was not associated with PD-L1 status. The majority of AEs were from GC; immune-related AEs were seen in 3 patients but none required steroids. ${ }^{55}$ who underwent surgery. Twelve percent and $2 \%$ of patients had grade 3 and grade 4 durvalumab-related AEs, respectively. ${ }^{56}$

NIAGARA (NCT03732677) is a randomized, open-label, global phase 3 trial of efficacy and safety of durvalumab in combination with GC as neoadjuvant therapy followed by durvalumab alone in the adjuvant setting in MIBC. The trial is still recruiting..$^{57}$

The Aura trial (NCT03674424), currently recruiting, is an open-label, interventional, randomized phase 2 study investigating the safety and efficacy of avelumab in combination with chemotherapy in the adjuvant setting in patients with MIBC, both cisplatin eligible and ineligible.

\subsection{Neoadjuvant ICIs in combination with targeted therapy in MIBC}

Olaparib inhibits the DNA repair enzyme PARP and has been approved in ovari-

\section{There are indeed a wealth of agents and trials, but the meaningful end points and outcomes of $O S$, patient-reported quality of life, and pathological response rates, depending on clinical stage, remain critical to determining the best treatment options for our patients.}

The SAKK 06/17 trial is another open-label, single-arm phase 2 study, enrolling 61 patients who had MIBC and were cisplatin eligible, cT2-T4a cN0-1. Four cycles of preoperative GC were administered, with durvalumab added starting with cycle 2 . Durvalumab was then continued after surgery every 4 weeks for 10 cycles. The primary end point was event-free survival at 2 years, with $\mathrm{PaIR}$ as the secondary end point. Interim results showed that the PaIR rate was $60 \%$, with $34 \%$ pCR in 53 patients an, breast, and prostate cancer treatment. It has been hypothesized that PARP inhibitors can augment the tumor mutational burden, making bladder cancer more immunogenic by increasing neoantigen production and upregulating PDL1 expression. ${ }^{58}$ The NEODURVARIB trial (NCT03534492) was a single-arm phase 2 study designed to assess the impact of neoadjuvant treatment with the combination of durvalumab plus olapar$\mathrm{ib}$ in patients with resectable MIBC (cT2T4a). Six to 8 weeks prior to planned cystectomy, patients received durvalumab $1500 \mathrm{mg}$ intravenously every 4 weeks for up to 2 months and olaparib $300 \mathrm{mg}$ twice a day orally for up to 56 days. Preliminary data showed that 10 patients $(50 \%)$ had a pCR, which was very promising. However, 3 patients developed grade 4 AEs including wound evisceration, hemorrhage, and septic shock. ${ }^{58}$

Cabozantinib (Cabometyx) is a small molecule tyrosine kinase inhibitor (TKI) targeting c-Met, VEGFR2, AXL, and RET. Cabozantinib in combination with atezolizumab resulted in favorable responses in patients with mUC in the phase $1 \mathrm{~b}$ COSMIC-021 trial (NCT03170960). ${ }^{59}$ Currently, this combination is being studied in the adjuvant setting. The ABATE trial (NCT04289779), currently recruiting, is a single-arm, open-label phase 2 study investigating the efficacy of cabozantinib and atezolizumab in patients with resectable MIBC who are ineligible for or decline cisplatin-based therapy. In this trial, atezolizumab $1200 \mathrm{mg}$ is given every 3 weeks along with cabozantinib $40 \mathrm{mg}$ daily. Each cycle is 21 days, and 3 cycles will be given before RC.

The promising results from the combination of linrodostat, the IDO1 inhibitor, and nivolumab in the mUC setting have led to the current study of linrodostat in the ENERGIZE trial (NCT03661320), a randomized, 3-arm phase 3 trial of neoadjuvant chemotherapy alone ( $\operatorname{arm} \mathrm{A}$ ) or chemotherapy plus nivolumab ( $\operatorname{arm} B$ ) or chemotherapy plus nivolumab with linrodostat (arm C) for cisplatin-eligible patients with MIBC. The trial was designed to evaluate and compare the efficacy and safety of this regimen followed by adjuvant nivolumab alone (arm B) or nivolumab and linrodostat (arm C). ${ }^{60}$

\subsection{Neoadjuvant ICIs with ADCs}

The ADC EV was approved in December 2019 based on the promising results of a phase 2 study in the metastatic setting. Consequently, 2 open-label, randomized phase 3 trials involving EV in the neoadjuvant setting opened and are underway. These 
TABLE 5. Ongoing Phase 3 Neoadjuvant 10 Trials in MIBC

\begin{tabular}{|c|c|c|c|c|}
\hline Trials & Eligibility stage & Cisplatin eligible & Arms & Primary end points \\
\hline \multicolumn{5}{|c|}{ Dual ICls } \\
\hline - NCT04209114 & - T2-T4a, N0, M & - No & $\begin{array}{l}\text { 1. Nivo+bempeg } \rightarrow \text { surgery } \\
\rightarrow \text { nivo+bempeg } \\
\text { 2. Nivo } \rightarrow \text { surgery } \rightarrow \text { nivo }\end{array}$ & $\begin{array}{l}\text { - } \mathrm{pCR} \\
\text { - EFS }\end{array}$ \\
\hline \multicolumn{5}{|c|}{ ICI combination with chemotherapy } \\
\hline - KEYNOTE-886 & $\begin{array}{l}\text { - T2-T4aN0M0 or } \\
\text { T1-T4aN1M0 }\end{array}$ & - Yes & $\begin{array}{l}\text { 1. Pembro+gem/cis } \rightarrow \text { surgery } \\
2 \text { Placebo }+ \text { gem/cis } \rightarrow \text { surgery }\end{array}$ & $\begin{array}{l}\text { - } \mathrm{pCR} \\
\text { - EFS }\end{array}$ \\
\hline - NIAGARA & - T2-T4aN0/1M0 & - Yes & $\begin{array}{l}\text { 1. Durva }+ \text { gem/sis } \rightarrow \text { surgery } \\
\text { 2. Gem/cis } \rightarrow \text { surgery }\end{array}$ & $\begin{array}{l}\text { - } \mathrm{pCR} \\
\text { - EFS }\end{array}$ \\
\hline - ENERGIZE & $\begin{array}{l}\text { - T2-T4a, N0 } \\
(<10 \mathrm{~mm} \text { on CT or } \\
\text { MRI), M0 }\end{array}$ & - Yes & $\begin{array}{l}\text { 1. Gem/cis } \rightarrow \text { surgery } \\
\text { 2. Nivo+gem/cis+linrodostate } \rightarrow \\
\text { surgery } \rightarrow \text { nivo+linrosostate } \\
\text { 3. Nivo+gem/cis+pbo } \rightarrow \text { surgery } \\
\rightarrow \text { nivo+pbo }\end{array}$ & $\begin{array}{l}\text { - pCR } \\
\text { - EFS }\end{array}$ \\
\hline \multicolumn{5}{|c|}{ ICI combination with antibody-drug conjugate } \\
\hline $\begin{array}{l}\text { - MK-3475-905/ } \\
\text { KEYNOTE-905/ EV-303 }\end{array}$ & $\begin{array}{l}\text { - cT2-T4aN0M0 or } \\
\text { cT1-T4aN1M0 }\end{array}$ & - No & $\begin{array}{l}\text { 1. Pembro } \rightarrow \text { surgery } \rightarrow \text { pembro } \\
\text { 2. Surgery alone } \\
\text { 3. Pembro+EV } \rightarrow \text { surgery } \\
\quad \rightarrow \text { pembro+EV }\end{array}$ & $\begin{array}{l}\text { - } p C R \text { in all participants } \\
\text { and } C P S \geq 10 \\
\text { - EFS in all participants } \\
\text { and } C P S \geq 10\end{array}$ \\
\hline $\begin{array}{l}\text { - MK-3475-B15/ } \\
\text { KEYNOTE-B15/ EV-304 }\end{array}$ & $\begin{array}{l}\text { - T2-T4aN0M0 or } \\
\text { T1-T4aN1M0 }\end{array}$ & - Yes & $\begin{array}{l}\text { 1. EV+pembro } \\
\text { 2. Gem/cis }\end{array}$ & $\begin{array}{l}\text { - pCR } \\
\text { - EFS }\end{array}$ \\
\hline
\end{tabular}

Bempeg, Benpegaldesleukin CPS, combined positive score; durva, durvalumab; EFS, event-free survival; EV, enfortumab vedotin; gem/cis, gemcitabine/cisplatin; ICl, immune checkpoint inhibitor; IO, immuno-oncology; nivo, nivolumab; pbo, placebo; pCR, pathological complete response; pembro, pembrolizumab.

are MK-3475-905/KEYNOTE-905/ EV-303 (NCT03924895), which will evaluate perioperative pembrolizumab vs perioperative EV in combination with pembrolizumab vs cystectomy alone in cisplatin-ineligible patients with MIBC; and MK-3475-B15/KEYNOTE-B15/EV304 (NCT04700124), which evaluates perioperative $\mathrm{EV}$ in combination with pembrolizumab vs neoadjuvant chemotherapy in cisplatin-eligible patients with MIBC (Table 5).

In response to the promising data from TROPHY-U-01, there is a planned phase 2 trial evaluating neoadjuvant sacituzumab govitecan alone or in combination with pembrolizumab before RC (SURE trial). ${ }^{61}$

\section{High-risk NMIBC}

Approximately $70 \%$ of patients present with NMIBC such as pTa (70-75\%), carcinoma in situ (CIS; $10 \%$ ), and pT1
$(15 \%)$ disease. Patients are risk stratified as low, intermediate, and high risk depending on pathological stage, grade, variant histology, size, and focality. Treatment and surveillance is based on risk stratification following transurethral resection of the bladder tumor and intravesical therapy with BCG and/or chemotherapy. Patients who are BCG unresponsive, candidates for early cystectomy, and at risk for progression to MIBC are the focus of investigational studies. BCG-unresponsive patients include those with refractory and BCG-relapsing cases; these are defined as persistent high-grade disease at 6 months despite adequate treatment and those with recurrence of high-grade disease after achieving a disease-free state at 6 months after adequate BCG, respectively. ${ }^{62}$

In January 2020, the FDA approved pembrolizumab based on the data showing that cohort A of KEYNOTE-057 (NCT02625961) demonstrated a $40.6 \% \mathrm{CR}$ at 3 months, with a median DOR of 16.2 months. ${ }^{63}$ SWOG 1605 (NCT02844816) showed atezolizumab provided a similar response in patients with BCG-unresponsive highrisk NMIBC. The CR rate was $41.1 \%$ (95\% CI, 29.7\%-53.2\%) at 3 months and $26.0 \%(95 \% \mathrm{CI}, 16.5 \%-37.6 \%)$ at 6 months. ${ }^{64}$ Trials are currently ongoing with durvalumab monotherapy in BCG-refractory CIS of the bladder (NCT02901548) and in NMIBC (NCT03759496).

\subsection{ICls in combination with BCG in high-risk NMIBC}

The possible roles of immune checkpoint signaling in BCG-resistant NMIBC ${ }^{65,66}$ and the promising data of ICI monotherapies ${ }^{64,67}$ suggest that combining ICIs with BCG therapy may increase the response rate. Several randomized, open-label trials are currently ongoing, including KEYNOTE-676 (NCT03711032), a phase 3 study of BCG with or without 
pembrolizumab in patients with highrisk NMIBC $^{68}$; ALBAN (NCT03799835), a phase 3 trial evaluating the efficacy of atezolizumab in combination with 1-year BCG bladder instillation in BCGnaïve, high-risk NMIBC; POTOMAC (NCT03528694), a phase 3 trial investigating durvalumab and BCG vs BCG alone in high-risk, BCG-naïve NMIBC; and CheckMate 9UT (NCT03519256), a phase 2 study of nivolumab monotherapy, nivolumab plus linrodostat mesylate, or nivolumab plus BCG in BCG-unresponsive, high-risk NMIBC. We await the results of these still-accruing trials.

\section{Discussion}

The emerging data for ICI combinations in bladder cancer are exciting, with the potential to change the therapeutic landscape for all patients with this disease. Up to $50 \%$ of patients with mUC are platinum ineligible or decline chemotherapy in the first-line setting, and ICI monotherapy has allowed these patients to receive systemic therapy; some have achieved a durable response with lower toxicity. This is tempered by the finding that patients with tumors with low or no PD-L1 expression might not benefit, and for platinum-eligible patients, chemotherapy remains the SOC. In the second-line setting, ICIs have demonstrated survival benefit over chemotherapy. We now have EV, sacituzumab govitecan, and erdafitinib to be used in the third- or fourth-line setting depending on FGFR2/3 mutational status and other clinical factors such as patient preference and toxicity profile.

As outlined in this paper, the development of regimens that combine ICIs with other IO drugs (eg, anti-CTL-4 agents), chemotherapy (including ADCs), targeted therapy (eg, FGFR inhibitors, PARP inhibitors, multitargeted TKIs), and immune suppressors to overcome ICI resistance (eg, IDO1 inhibitors) is ongoing. The most promising regimen to date is the combination of EV with pembrolizumab, with a response rate of more than $70 \%$ and a CBR of $90 \%$ in patients who are cisplatin ineligible, regardless of PD-L1 status. This regimen is now being compared with chemotherapy in the first-line setting in platinum-eligible patients and in the muscle-invasive setting.

\section{The emerging data for ICI combinations in bladder cancer are exciting, with the potential to change the therapeutic landscape}

In the muscle-invasive setting, the response rates to anti-PD-1/L1 as single agents when combined with other IO drugs (eg, anti-CTLA-4 agents), or with targeted therapy and chemotherapy, all appear to achieve pCR and pathological non-muscle-invasive rates that compare favorably with historical controls. If confirmed in larger randomized trials, these results will give patients who have not been able to receive chemotherapy in this setting an opportunity to receive therapy. The results of the CheckMate 274 trial (NCT02632409) show disease-free survival benefit in the adjuvant setting with nivolumab in patients with and without prior neoadjuvant therapy who demonstrate high-risk pathological features for recurrence. Again, the goal here is the ability to expand therapeutic options for patients, which will hopefully translate to improvement in OS with less toxicity. An additional advantage of neoadjuvant therapy is the ability to include biomarker analysis to help predict which patients might benefit. Beyond PDL1, other predictive and prognostic biomarkers need to be investigated

ICIs are tools in the spectrum of treatments being investigated to improve outcomes in high-risk NMIBC. ${ }^{69}$ Novel intravesical therapies-including recombinant adenovirus gene transfer vector; oportuzumab monatox (Vicinium; a recombinant fusion protein drug consisting of an epithelial cell adhesion molecule-specific antibody fragment linked to a pseudomonas exotoxin $\mathrm{A})^{70}$; and combination therapy along with BCG in the earlier setting-can potentially reduce progression to muscle-invasive disease and to recurrent high-risk disease, as well as reduce the need for early cystectomy.

There are indeed a wealth of agents and trials, but the meaningful end points and outcomes of OS, patient-reported quality of life, and pathological response rates, depending on clinical stage, remain critical to determining the best treatment options for our patients.

FINANCIAL DISCLOSURE: JV receives research funding at her institution for clinical trials from Merck, Bristol Meyers Squibb, Genentech, Astellas, Seattle Genetics, Astra Zeneca.

CONFLICTS OF INTEREST: JV serves on advisory boards for AstraZeneca, Bristol Meyers Squibb, and Seattle Genetics

For full reference list, visit cancernetwork.com/Feng_7.21

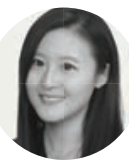

Feng is a hematology and oncology fellow at the Knight Cancer Institute of the Oregon Health \& Science University in Portland, OR.

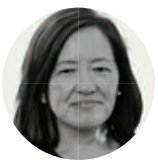

Vuky is an associate professor and Program Director of Genitourinary (nonProstate) Oncology at the Knight Cancer Institute, Oregon Health \& Science University in Portland, OR. 


\section{Median Lobe Urethral Embolus After Focal Ablation of Gleason 7 Prostate Cancer}

Alan Makedon, BA; Paul Maroni, MD

ABSTRACT: This is the case of a man, aged 56 years, who presented with urinary intermittency, frequency, urgency, and dysuria 5 months after undergoing focal laser ablation (FLA) of Gleason $3+4=7$ prostate cancer (PC). Cystoscopy revealed a foreign body obstruction of the bladder and the patient experienced immediate relief after its removal. Final pathology confirmed the diagnosis of the foreign body as a piece of necrotic prostatic tissue originating from the median lobe. To our knowledge, this is the first case of intermittent urethral obstruction by a sloughed median prostatic lobe following FLA. FLA is an emerging therapy for low- or intermediate-grade PCs, and this case highlights the need for continued evaluation of long-term outcomes of this procedure.

KEY POINTS: focal therapy, focal laser ablation, prostate cancer, foreign body obstruction, median lobe embolus

\section{Background}

Focal laser ablation (FLA) is a technology whose use is emerging in the management of small, low- or intermediate-risk prostate cancers (PCs). Although the gold-standard therapies for the management of PC are active surveillance, radical prostatectomy, and/or radiation therapy, several contemporary studies have shown the benefit of FLA in the reduction of urinary and erectile adverse effects (AEs) while adhering to oncologic principles. ${ }^{1}$ FLA uses MRI imaging guidance and temperature monitoring with a laser platform in order to achieve accurate and homog- enous prostate tissue ablation. ${ }^{2}$ In this case report, we describe a patient who presented to our clinic with intermittent obstructive urinary symptoms 5 months after his FLA for Gleason 3+4=7 PC.

\section{Case Presentation}

We present a male, aged 56 years, with an elevated prostate-specific antigen (PSA) of 6.2 who was found to have Gleason 6 PC on systematic biopsy. Approximately 6 months later, MRI identified a Prostate Image-Reporting and Data System (PI-RADS) 4 lesion in the right base to mid-gland transition zone, and target- ed biopsy upstaged his cancer to Gleason $3+4=7$ (Figure 1). After he consulted with numerous physicians, he underwent uncomplicated FLA of his prostate.

Five months after the procedure, he presented to the clinic with urinary urgency, frequency, dysuria, and a complaint of sudden stoppage of urinary flow midstream. His American Urological Association (AUA) symptom score was 22 on tamsulosin. Cystoscopy revealed a foreign body (FB) with the appearance of partially calcified tissue, measuring approximately $1 \mathrm{~cm}$, in the bladder. Careful inspection of the patient's pretreatment and posttreatment MRI demonstrated loss of a median prostate lobe after treatment, with a nodular FB in the bladder (Figure 2A and 2B). The FB was removed endoscopically under anesthesia in multiple pieces using cold-cup biopsy forceps. The patient experienced immediate improvement in his urinary complaints. Final pathology demonstrated a small, detached fragment of granulation tissue admixed with fragments of stone. He had immediate resolution of symptoms, and his postoperative AUA symptom score was 6 . Seven months after the FLA procedure, he had a PSA of 1.6.

\section{Discussion}

Over the last decade, there has been an increase in novel techniques for management of localized PC. FLA, cryoablation (CA), and focal therapy, using high-inten- 
sity focused ultrasound (HIFU), ${ }^{3}$ all offer minimally invasive and organ-sparing options for such low- or intermediate-grade, unifocal cancers. Specifically, FLA embodies many benefits of a minimally invasive procedure for the treatment of localized PC, as it has minimal impact on quality of life and reasonable oncologic control with few AEs. ${ }^{4}$ Given the novelty of the procedure, some rare complications related to FLA may emerge over time.

Long-term outcomes of focal therapies such as HIFU and cryotherapy have been reported. One such systematic review and meta-analysis of HIFU revealed that following wholegland HIFU, the incidence rates of urinary obstruction, retention, and infection were $15 \%, 11 \%$, and $7 \%$, respectively. The incidence rates of urinary obstruction, retention, and infection following partial-gland HIFU were $2 \%, 9 \%$, and $11 \%$, respectively. ${ }^{5,6}$ The differences in complication rates between wholeand partial-gland prostate ablation reflect the need for ongoing analysis of therapy options for localized PC. A study of cryosurgical ablation (CSA) for patients with low-grade PC found that the most common AE 1 year post CSA was urinary tract obstruction, sometimes necessitating the removal of necrotic prostatic tissue and calcifications. ${ }^{7}$ Although FB obstruction of the bladder complications have been reported following HIFU and CSA, they have not been addressed or studied in FLA.

A median lobe bladder FB embolus is an extraordinarily unusual complication after an ablative treatment of the pros-

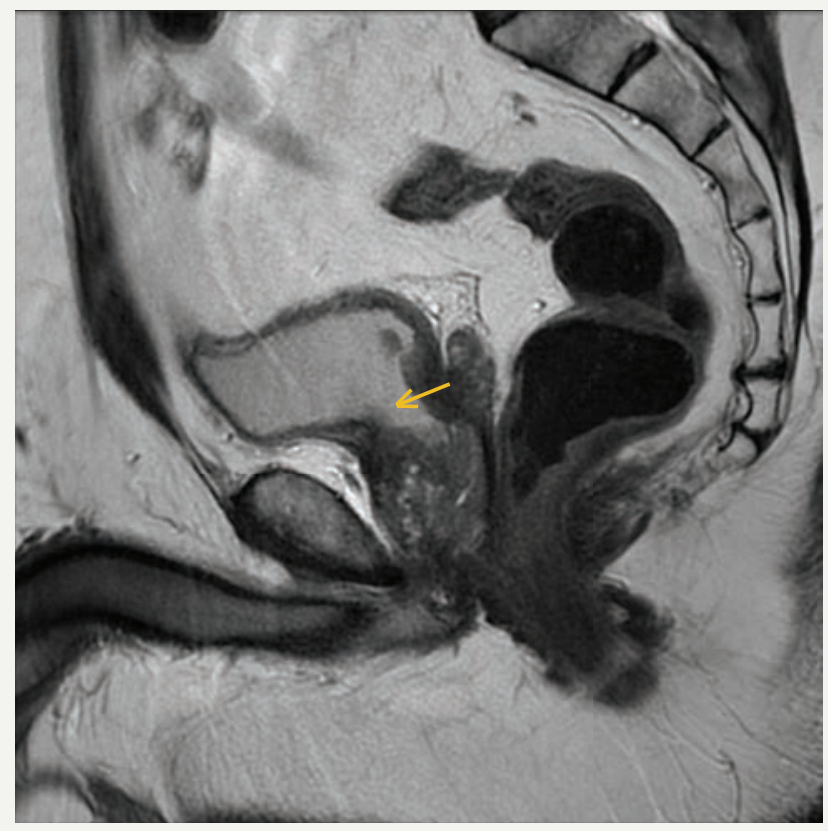

FIGURE २A. Sagittal T2 After FLA Showing Ablated

\section{Prostate (arrow).}

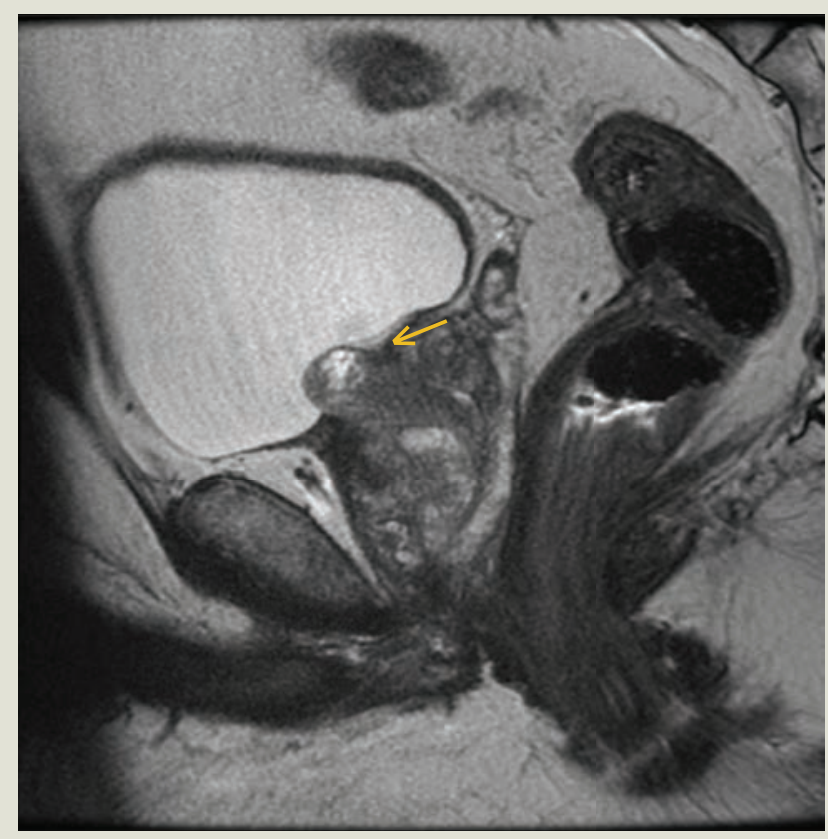

FIGURE 1. Sagittal T2 With PI-RADS-4 Lesion in the Right Base to Midgland Transition Zone (arrow).

PI-RADS: Prostate Image - Reporting and Data System.

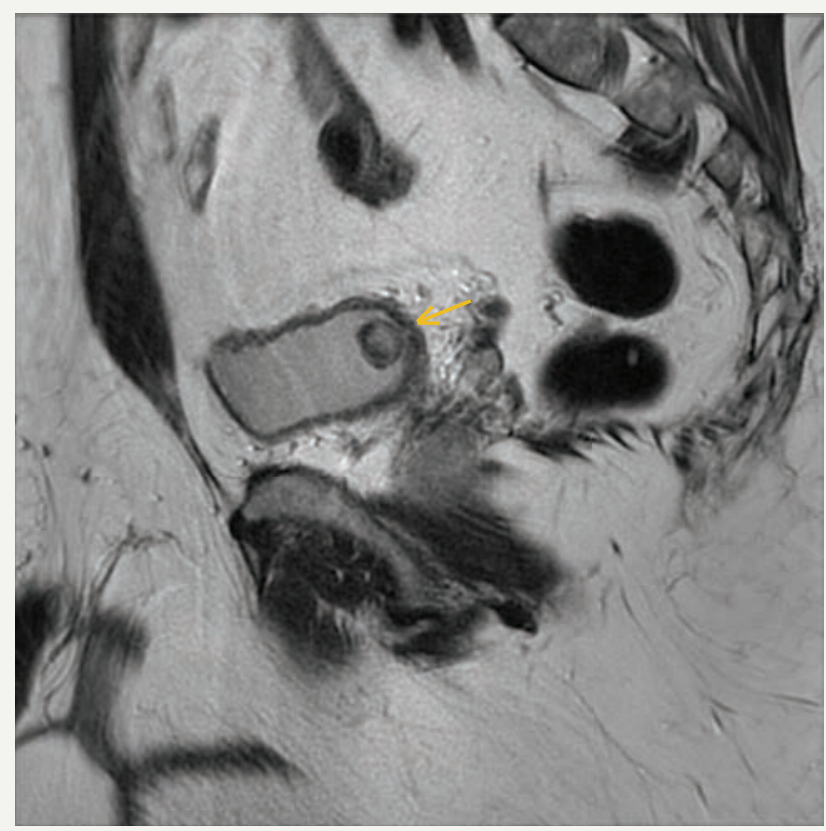

FIGURE 2B. Sagittal T2 After FLA Showing Presence of FB, Alternate View (arrow).

FB: foreign body; FLA: focal laser ablation; PI-RADS: Prostate Image - Reporting and Data System. 
tate. Presumably, the FLA caused necrosis of the base of the median lobe through destruction of the proximal blood supply, resulting in amputation of the median lobe. This FB intermittently embolized into the prostatic urethra/bladder neck causing sudden cessation of urine flow accompanied by irritative voiding symptoms. We can find no other instance of a similar complication related to ablative therapies of the prostate in the searchable literature.

Given the peculiar nature of this complication and the likely increased utilization of ablative procedures for prostate disease in future care, endoscopic evaluation with cystoscopy may be an earlier step in the management of urinary morbidity after these treatments. Urethral sloughing after HIFU and CA is a more common cause of urethral obstruction, and cystoscopy may be both diagnostic and therapeutic. Checking for urinary infection would be best clinical practice, but medical management would not have helped this patient. We do not think that those patients with protuberant median lobes should be denied ablative treatments for prostate disease, but perhaps a higher index of suspicion would be prudent if patients experience urinary issues post treatment.

This also begs the question of whether FLA would be a reasonable treatment for benign prostate enlargement. The defect present on his posttreatment MRI was similar to a defect related to transurethral resection of the prostate, and, compared with what he was experiencing prior to FLA, the patient had notable improvement in his benign prostatic hyperplasia symptomatology.

To our knowledge, this is the first case of prostatic tissue obstructing the bladder following FLA of the prostate. This case highlights the necessity for long-term assessment of outcomes and complications following emerging focal therapies, specifically laser ablation.

\section{FINANCIAL DISCLOSURE: PM: Astellas}

(investigator), Dendreon (investigator)

$\rightarrow$

For full reference list, visit:

cancernetwork.com/Makedon_7.21

\section{Makedon}

is an MD candidate at the University of Colorado School of Medicine in Aurora, CO.

\section{Maroni}

is an associate professor of surgery-urology in the Department of Surgery and Urologic Oncology Program Lead at the the University of Colorado Anschutz Medical Campus in Aurora, CO.

\section{ONCOLOGY}

\section{Now Accepting Original Research Articles For Publication}

Authors are encouraged to submit high-quality, original clinical trial manuscripts and investigations.

\section{Benefits of publishing in ONCOLOGY}

- Gain access to a diverse audience of multidisciplinary oncology professionals

- Broaden the impact of your research through print, digital, and audio channels

- Opportunity to be featured on the ONCOLOGY ${ }^{\circledR}$ Peer Review On-The-Go bi weekly podcast

All research is PubMed indexed and double-blind peer reviewed. Authors wishing to submit their original research manuscripts are encouraged to review the $O N C O L O G{ }^{\circledR}$ author guidelines by visiting:

\section{cancernetwork.com/call-for-papers}

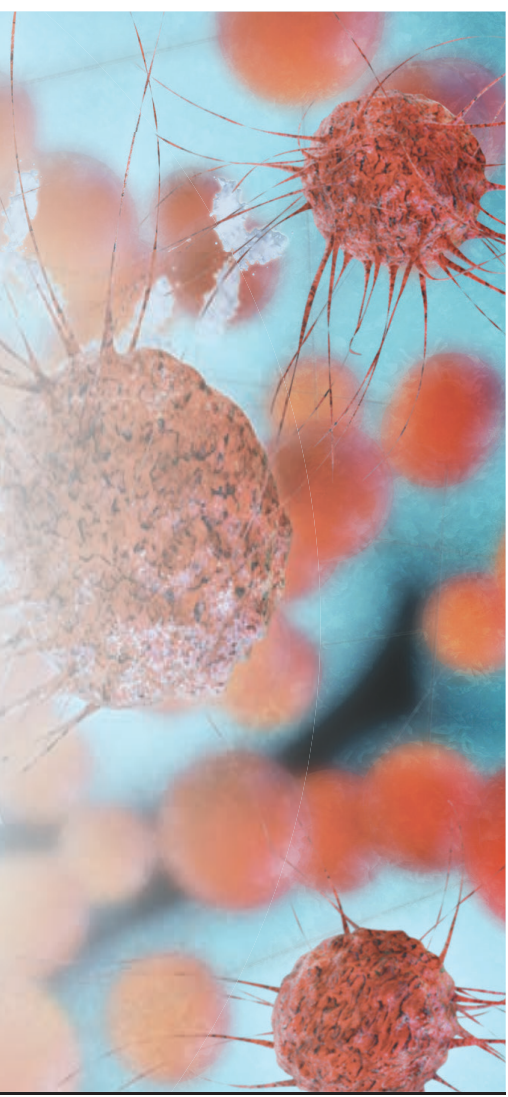




\section{Advanced Penile Cancer Presenting With Renal Failure}

Sarah Derby, MBChB' ; Jan Wallace, MBChB2; Balaji Venugopal, MBBS MD3; Maria Almudena Cascales Garcia, MB Msc²

'Wolfson Wohl Cancer Research Centre, Institute of Cancer Sciences, University of Glasgow, Scotland, United Kingdom

${ }^{2}$ Beatson West of Scotland Cancer Centre, United Kingdom ${ }^{3}$ University of Glasgow, Scotland, United Kingdom

\section{THE \\ CASE}

"MK," a man aged 67 years, presented with fatigue and nausea to his primary care physician. Routine blood tests showed a stage III acute kidney injury and the patient was admitted urgently into the hospital for investigation. On initial assessment by the nursing staff, with urine dip and bladder scan, he was found to have a large fungating mass on his penis. Upon further questioning, MK reported having had trouble with urination for a number of years and that he had been concealing the mass for at least 1 year due to embarrassment. He required a suprapubic catheter as the mass had completely obstructed his urethra. Clinical examination revealed that the external component on the penile shaft was entirely destroyed by the tumor, with extension deep into the entirety of the scrotum, and perineal soft-tissue invasion was also apparent.

CT staging scans confirmed the primary tumor and a suspicious left 1.2-cm inguinal lymph node but no distant metastases. MRI of the pelvis revealed complete replacement of the penis with tumor as well as invasion into the scrotum and bilateral groin soft tissue; additionally, early pubic bone invasion was present, with left groin lymphadenopathy. Biopsy verified squamous cell carcinoma of the penis, and discussion with the multidisciplinary team uroradiologist confirmed bony invasion (Figure 1).

Due to the extent of disease and after local bony involvement was confirmed, the disease was deemed inoperable. Given MK's surprisingly limited disease and relative fitness otherwise, he was referred to oncology for assessment of fitness for treatment. It was felt that given the extent of disease and patient fitness, radiotherapy for local control might be the best option to palliate symptoms. Physicians considered concurrent and neoadjuvant chemotherapy, but because MK's renal failure was slow to improve, the concern was that the delay in time caused by chemotherapy might result in the radiotherapy field no longer being feasible if the cancer were to progress further.

Therefore, treatment was planned with 60 Gy in 30 fractions, with a 6-Gy boost to the primary site using volumetric arc technique, and the use of $1-\mathrm{cm}$ wax bolus covering the gross total vol-

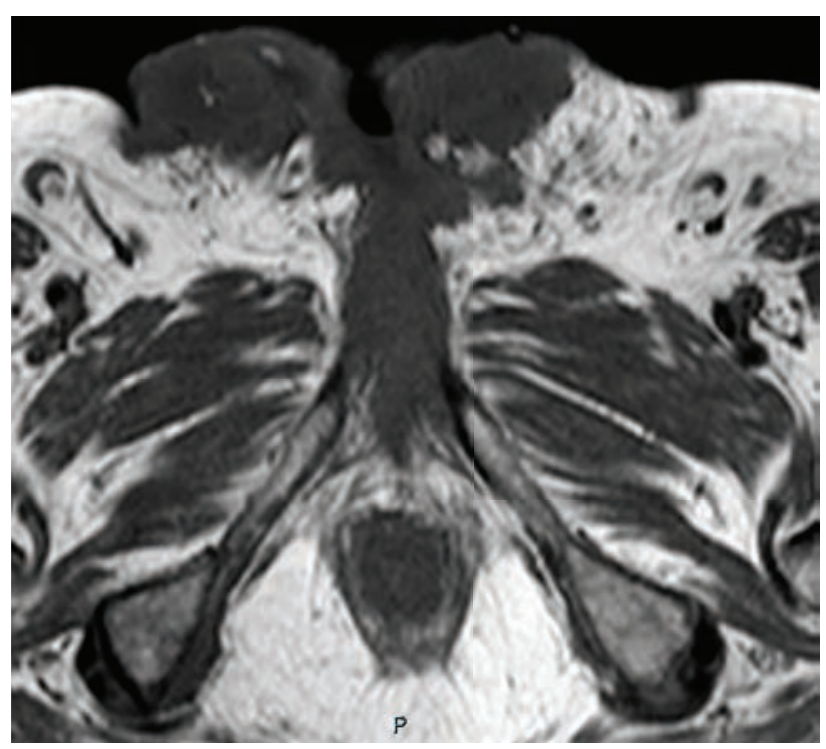

FIGURE 1. MRI of Penile Cancer Showing Early Invasion Into Bone and Destruction of Penile Anatomy

\section{What treatment options would you consider for this patient?}
A. Palliative bleomycin/vincristine/methotrexate chemotherapy
B. Maximal debulking of tumor including pelvic bone resection and reconstruction with pelvic and inguinal lymphadenectomy
C. High-dose radiotherapy +/- concurrent chemotherapy for long-term local control
D. Local debulking of the penis for palliation only
E. Single fraction of radiotherapy for palliation only

TURN TO PAGE 426 for the answer and a discussion of this case by experts. 


\section{CORRECT ANSWER: C}

\section{Continued from page 425}

\begin{abstract}
Answer
A. While palliative or neoadjuvant chemotherapy may be an appropriate option, bleomycin/ methotrexate/cisplatin, which has been used neoadjuvantly in penile cancer, may result in high levels of bleomycin-related toxicity. ${ }^{1}$
\end{abstract}

B. Debulking in this patient would be an extremely morbid surgery, requiring bony resection; it would also likely result in positive margins and so would not be appropriate. Surgical treatment is discussed later in the article, including management of pelvic and inguinal lymph nodes.

\section{Correct}

D. Palliative local debulking for penile cancer may be appropriate in some circumstances, but this case displays extensive cutaneous and bony involvement. Therefore, local debulking is likely to result in positive margins and poor wound healing, potentially causing further morbidity in what is likely to be a palliative case.

E. While palliative radiotherapy may be an appropriate treatment option, a single 8 Gy fraction is unlikely to be effective given the large area requiring treatment.

ume of the penis and nodes plus margin (Figure 2). MK successfully completed radical radiotherapy with acute grade 2 radiation dermatitis. Initial scans showed a response to treatment, although some of the necrotic residual tumor bed was slow to resolve. The suprapubic catheter was required for ongoing urinary diversion as the tumor shrinkage had essentially resulted in a loss of any normal penile anatomy (Figure 3). Scans continued to show an improvement in the appearance of the tumor bed until there was no discernible remaining cancer. The patient has remained on follow-up for 2 years with no evidence of distant disease or local progression. Of interest, MK developed a transient viral human papillomavirus (HPV) infection in the form of warts at the scrotum 18 months after completing radiotherapy and went on to have HPV immunization.

\section{Background}

Penile cancer incidence in developed countries is rare, and management requires careful consideration. In the United Kingdom, it accounts for only about $0.2 \%$ to $0.6 \%$ of cancers in men and 2.9 new cases per year per 100,000 in Scotland..$^{2,3}$ Similarly, in the United States, reported incidence is low at 0.81 per $100,000 .{ }^{4}$ However, in developing countries the incidence can be significantly higher, with incidence of up to 6.15 per 100,000 in such localities as Maranhão, Brazil. ${ }^{5}$ Evidence indicates, however, that incidence may be increasing in developed countries: For example, in Saxony, Germany, reported incidence has increased from 1.2 per 100,000 in 1961 to 1.8 per 100,000 in $2012 .^{6}$

Risk factors for penile cancer that have been investigated include age, sexual history, and socioeconomic status. ${ }^{3}$ Historically, penile cancer has been considered primarily a disease of the elderly; however, evidence increasingly supports that a significant proportion of cases occur in younger men; a prospective study showed that a quarter of patients were diagnosed when aged less than 50 years. ${ }^{7}$ This may relate to cases driven by HPV, as it is known that HPV-related cancer incidence has generally increased in past decades and prior sexual history can play an important role in this particular cancer subgroup. ${ }^{8}$ Interestingly, HPV-positive disease may be associated with better survival, a phenomenon that has also been seen in head-and-neck and oropharyngeal cancers. ${ }^{9}$ The proportion of penile cancers that are HPV driven is lower than in other related groups, such as cervical cancer, but up to one-third of penile cancers may be HPV related. ${ }^{10,11}$ Some penile cancers are associated with inflammation or chronic inflammatory processes, as chronic inflammatory processes including balanitis, phimosis, and lichen sclerosis increase penile cancer risk. Smoking is another risk factor. ${ }^{12}$ Interestingly, neonatal circumcision appears to be associated with a reduced risk of penile cancer. ${ }^{13}$ Lower socioeconomic status has also been associated with an increased risk of penile cancer, and low educational level has also been associated with later presentation of more advanced primary tumors. ${ }^{14}$ Increased rates of penile cancer have been noted in patients of lower socioeconomic backgrounds, both in developing and developed countries. ${ }^{14,15}$

\section{Presentation and Staging}

Staging of penile cancer is important (Table). The factor that most strongly influences survival and outcome is lymph node staging; 5 -year survival is only $25 \%$ in patients with 3 or more positive lymph nodes compared with $77 \%$ in patients with 2 or fewer positive nodes. ${ }^{16}$ Similarly, pelvic disease and bilateral disease are also associated with poor prognosis; there are few survivors at 5 years in the presence of pelvic nodes. ${ }^{17}$ Groin node dissection is therefore an important consideration in patients undergoing radical surgery. However, nodal dissection significantly increases the risk of complications such as lymphedema. ${ }^{17}$ Another complicating factor is the presence of clinically occult lymph node metastases, which affects a significant proportion (up to $20 \%$ ) of patients. ${ }^{18}$ While advanced-stage penile cancer can cause significant problems locoregionally, the risk of distant metastasis is low and tends to occur late in the course of disease. Metastatic disease at presentation is seen in less than $5 \%$ of patients. ${ }^{19}$

\section{Treatment \\ Early disease}

Penile cancer treatment varies depending on stage, site, and grade of disease. 
Early-stage T1 cancers that are grade 1 or 2 may be treated with conservative organ-preserving techniques such as surgery, but treatments have also included Mohs micrographic surgery, laser therapy, and radiotherapy in the form of interstitial implant brachytherapy. ${ }^{20-22}$ More than $90 \%$ of recurrences occur in the first 5 years, and patients therefore require close follow-up for locoregional recurrence, particularly in the first 2 years after follow-up when the risk of local recurrence is highest. ${ }^{23}$ In T1, grade 3 disease, surgery tends to require more extensive intervention and may require glansectomy with reconstruction with wider margins $(8 \mathrm{~mm})$, due to the high risk of regional spread. ${ }^{24,25}$ Depending on site, it may still be possible to maintain functionality for urination and in some cases sexual function. Brachytherapy and external beam therapy can also be considered but require close surveillance for recurrence. ${ }^{26,27}$ Brachytherapy can be an attractive option as it is superior to external beam treatment in providing organ preservation, particularly in $\mathrm{T} 1$ or T2 disease. ${ }^{28}$

\section{Locally advanced disease}

The management of locally advanced penile cancer is somewhat complex and can be controversial. In particular, management of lymph nodes can be a matter of debate. As previously mentioned, even clinically node-negative cancers can hide occult lymph node metastases, and for patients with T1b or greater disease, a modified lymph node dissection or sentinel node biopsy is recommended. ${ }^{29}$ In clinically node-positive disease, inguinal lymph node dissection is generally recommended, and consideration should be given to neoadjuvant chemotherapy in bulky or fixed N3 disease. ${ }^{30}$ Further, the presence of inguinal lymph nodes increases the risk of pelvic nodal involvement; the presence of 3 or more inguinal nodes has been associated with at least a 4-fold greater risk of pelvic nodal disease, with a rate of more than $20 \% .{ }^{31}$ The European Association of Urology (EAU) recommends pelvic lymph node dissection in all patients with 2 or more inguinal node metastases. ${ }^{32}$ Adjuvant chemotherapy may also be considered in $\mathrm{N} 2$ or N3 disease following lymphadenectomy; retrospective data support this and demonstrate good rates of disease-free survival compared with historical controls. ${ }^{33}$ Several chemotherapy regimens, including cisplatin/5-fluorouracil (5FU), have been proposed in the adjuvant and neoadjuvant settings. ${ }^{34,35}$ More recently, the more intensive paclitaxel/cisplatin/ifosfamide regimen showed a good objective response rate (ORR; 50\%) in the neoadjuvant setting, and 3 of 30 men had complete responses in a phase 2 trial (NCT00512096). ${ }^{36}$

\section{The role of radiotherapy}

The role of adjuvant radiotherapy remains a matter for debate, and management guidelines do not universally agree. The EAU penile cancer guidelines do not routinely recommend consideration of adjuvant radiotherapy; however, a number of centers propose a role for adjuvant radiotherapy, particularly in disease that may not be feasibly operable or has not responded to neoadjuvant chemotherapy. ${ }^{9,37-39}$ Considering radiotherapy also may have some advantage in cases of extracapsular spread, which is a particularly poor prognostic factor. ${ }^{40}$ In this controversial field of perioperative treatment, the InPACT trial (International Penile Advanced Cancer Trial, an International Rare Cancers Initiative study; NCT02305654) seeks to clarify the optimal sequencing of surgery in combination with chemotherapy and radiotherapy. ${ }^{41}$ Specifically, InPACT compares initial treatment through 3 arms: standard surgery, neoadjuvant chemo- therapy followed by standard surgery, and neoadjuvant chemoradiotherapy followed by standard surgery.

In some patients, surgery is not feasible due to the location of disease or its extent, or due to patient fitness or wishes. In these cases, radiotherapy can be considered with or without chemotherapy for aggressive local control, as demonstrated in MK's case. ${ }^{42}$

\section{Systemic palliative options}

Cisplatin tends to be the backbone for penile cancer treatment regimens. Tax-

\section{FIGURE 2. 95\% Isodose Coverage With Conformal Volumetric Arc Technique Treatment and Wax Bolus}

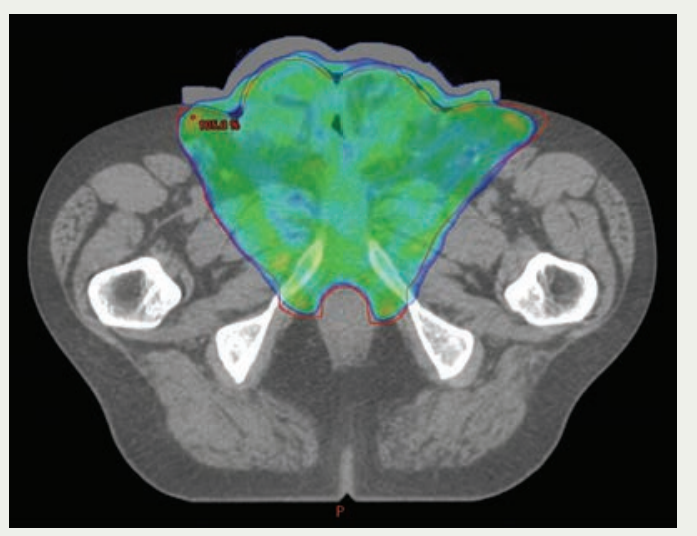

\begin{abstract}
FIGURE 3. MRI From Most Recent Follow-Up at 2 Years, Demonstrating No Disease
\end{abstract}

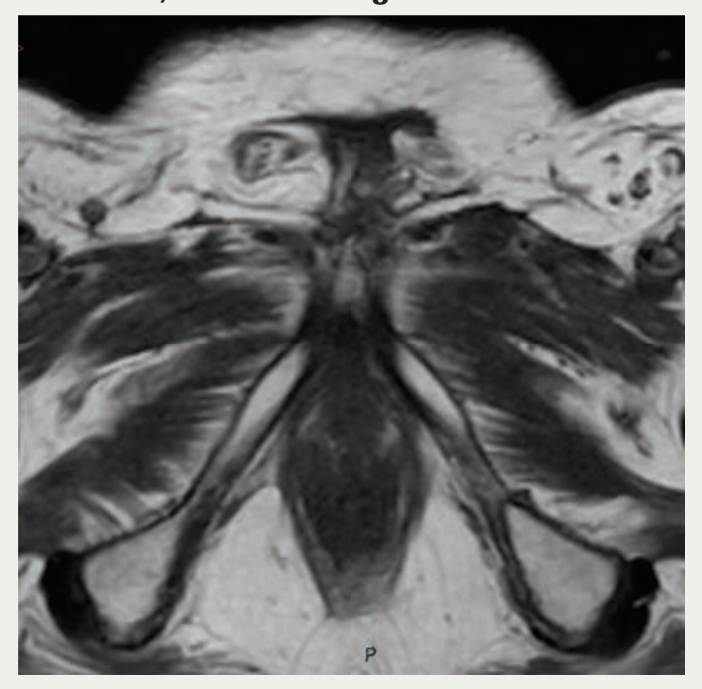




\section{TABLE. Penile CancerTnM Staging Classification}

\begin{tabular}{|c|c|c|c|}
\hline Stage & T stage & N stage & M stage \\
\hline Stage I & $\begin{array}{l}\text { T1a: invades subepithelial } \\
\text { connective tissue; grade } 1 \text { or } 2\end{array}$ & No & Mo \\
\hline Stage II & $\begin{array}{l}\text { T1b : grade } 3 \\
\text { T2 : invades corpus spongiosum } \\
\text { T3: invades corpus cavernosum }\end{array}$ & No & Mo \\
\hline Stage III & T1- T3 & $\begin{array}{l}\text { N1: metastasis in 1-2 } \\
\text { inguinal lymph nodes } \\
\text { N2: Metastasis in more } \\
\text { than } 2 \text { lymph nodes OR } \\
\text { bilateral lymph nodes }\end{array}$ & Mo \\
\hline \multirow[t]{2}{*}{ Stage IV } & $\begin{array}{l}\text { T4: invasion of adjacent } \\
\text { structures }\end{array}$ & No-2 & \multirow{2}{*}{$\begin{array}{l}\text { Or M1 } \\
\text { disease: } \\
\text { distant } \\
\text { metastasis }\end{array}$} \\
\hline & Any $T$ & $\begin{array}{l}\text { N3: pelvic lymph nodes } \\
\text { OR extranodal extension } \\
\text { of any lymph nodes }\end{array}$ & \\
\hline
\end{tabular}

TNM, tumor, node, metastasis from TNM Classification of Malignant Tumours (8th edition).

anes, however, have also demonstrated response. Still, because trials have been small, reported responses to treatment have been variable. ${ }^{43,44}$ The combination of docetaxel, cisplatin, and $5 \mathrm{FU}$ was evaluated in 29 patients with locally advanced or metastatic disease by the UK National Cancer Research Institute penile cancer subgroup; the overall ORR was $38.5 \% .{ }^{45}$ More recently, the VinCap trial (NCT02057913) evaluated vinflunine in locally advanced or metastatic penile cancer and reported a clinical benefit rate of $45.5 \%$. The authors concluded that further research could be of value with this novel vinca alkaloid. ${ }^{46}$ Other systemic options reviewed in penile cancer have included EGFR-targeted therapies such as cetuximab (Erbitux); a review of treatment with cetuximab, erlotinib (Tarceva), and gefitinib (Iressa) demonstrated response in $23.5 \%$ of patients. ${ }^{47}$

Penile cancer is rare, and like many rare cancers, it does not attract a great deal of investigation of novel agents. Therefore, treatments such as immunotherapy in penile cancer have not been rigorously tested. ${ }^{48}$ Indeed, 1 phase 2 trial attempted to investigate the use of pembrolizumab
(Keytruda) in penile cancer but was closed early due to poor recruitment (NCT02837042). Case reports of immunotherapy use in penile cancer have indicated that durable responses can be seen even after the failure of multimodal treatment. One report described a man, aged 79 years, with chemo- and radioresistant disease who showed durable response on atezolizumab. Further responses of up to 38 months were demonstrated in a case series using pembrolizumab. ${ }^{49,50}$ These case reports demonstrate the challenge of caring for patients with rare cancers as trials are expensive and difficult to recruit to. Results are eagerly awaited of current clinical trials that are assessing the efficacy of immunotherapy as monotherapy or in combination with radiotherapy in penile carcinoma (NCT03686332; NCT03391479). Our center also has experience in using palliative immunotherapy in penile cancer that generated objective response in otherwise treatment-resistant disease (unpublished data).

More broadly, the use of T-cell therapy in HPV-related cancers has been investigated with durable response rates. Although this is a majority cervical cancer population, treatment by molecular profiling may have application for HPV-related penile cancers. ${ }^{51}$ Participation in trials that distinguish disease by molecular subtype may be advantageous in penile cancer, due to its overlap with other squamous cancers and targetable pathways may be utilized. ${ }^{52}$

\section{Final Thoughts}

It is rare for specialists, much less primary care physicians, to see penile cancer. Disease heterogeneity and late presentation mean that treatment planning can be challenging and is often fraught with controversy. Cumulative investigation into the importance of HPV and of key driver mutations affecting prognosis is broadening our understanding of this disease. The crucial factor to improving outcomes in penile cancer will be clinical trial engagement and successful recruitment of patients, with utilization of early-phase and basket-design trials. An important step in recognizing the challenge of treating these rare cancers is the formation of the Global Society of Rare Genitourinary Tumors (www.GSRGT.com), created to promote evidence-based care.

FINANCIAL DISCLOSURE: The authors have no significant financial interest in or other relationship with the manufacturer of any product or provider of any service mentioned in this article.

\section{ABOUT THE SERIES EDITORS:}

Maria T. Bourlon, MD is associate professor, Head Urologic Oncology Clinic, national researcher. Instituto Nacional de Ciencias Médicas y Nutrición Salvador Zubirán. Mexico City, Mexico. She is also a member of ASCO's IDEA Working Group.

E. David Crawford, $M D$, is chairman, Prostate Conditions Education Council; editor in chief, Grand Rounds in Urology; and professor of urology, University of California San Diego, La Jolla, CA.

For full reference list, visit cancernetwork.com/Derby_7.21 


\section{cancervetwork}

Hear timely \& informative insights from some of the leading voices in the field.
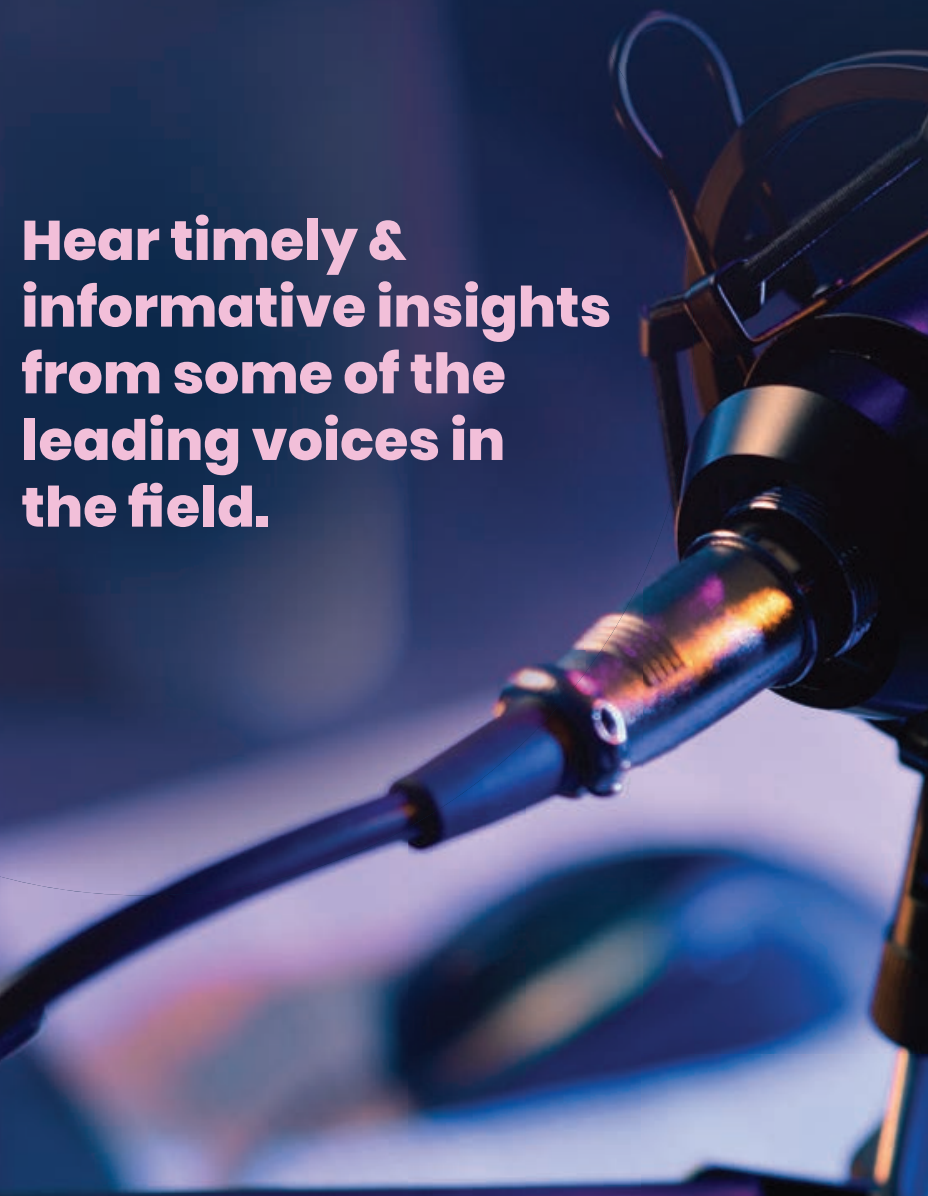

$\because$

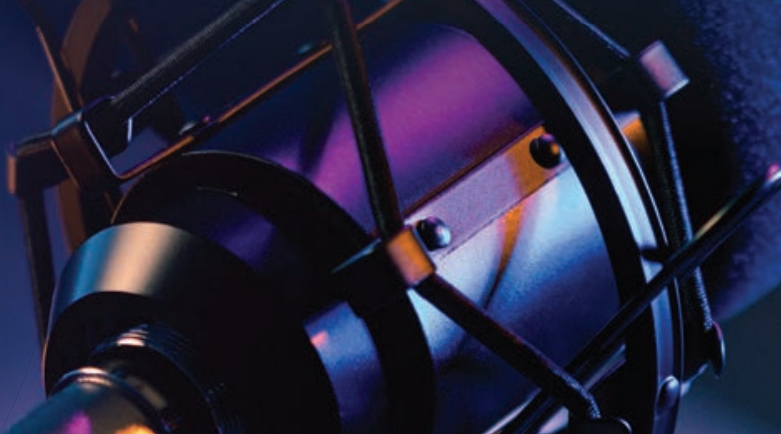

$\because$

Listen: www.cancernetwork.com/resources/podcasts

Sean to listen

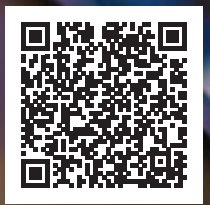




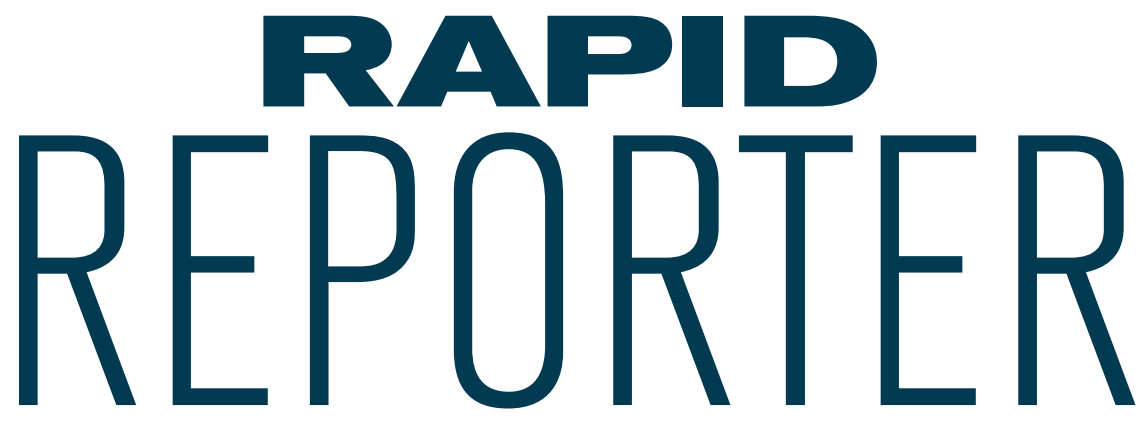

ONCOLOGY ${ }^{\circledR}$ Reviews Key Presentations From the 2021 American Society of Clinical Oncology Annual Meeting
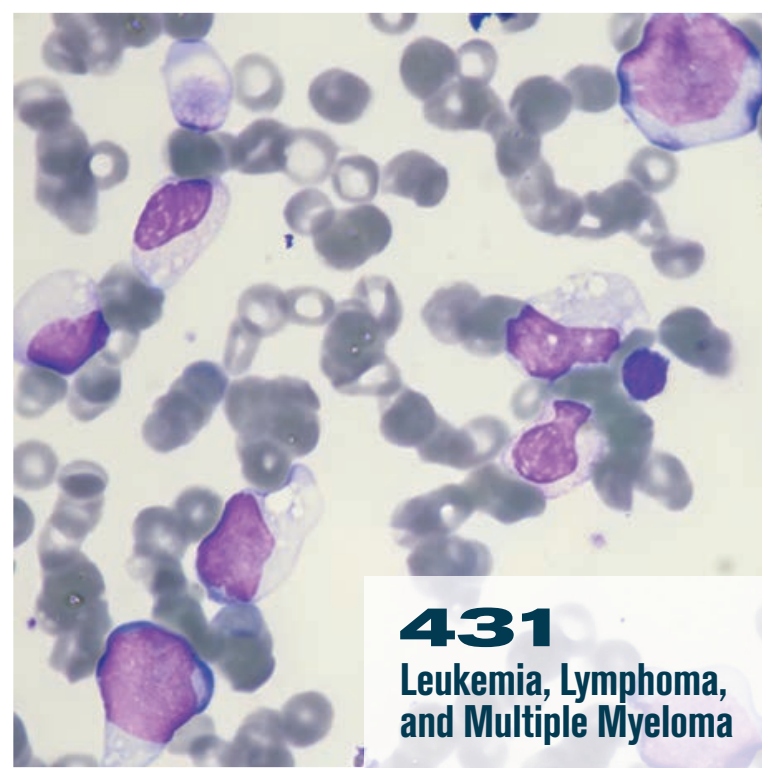

Leukemia, Lymphoma, and Multiple Myeloma

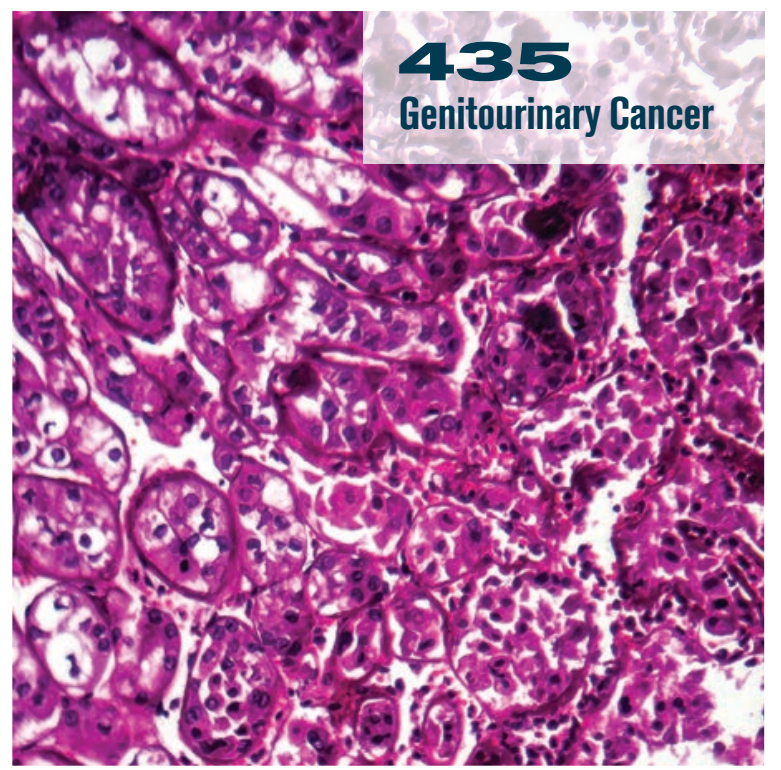

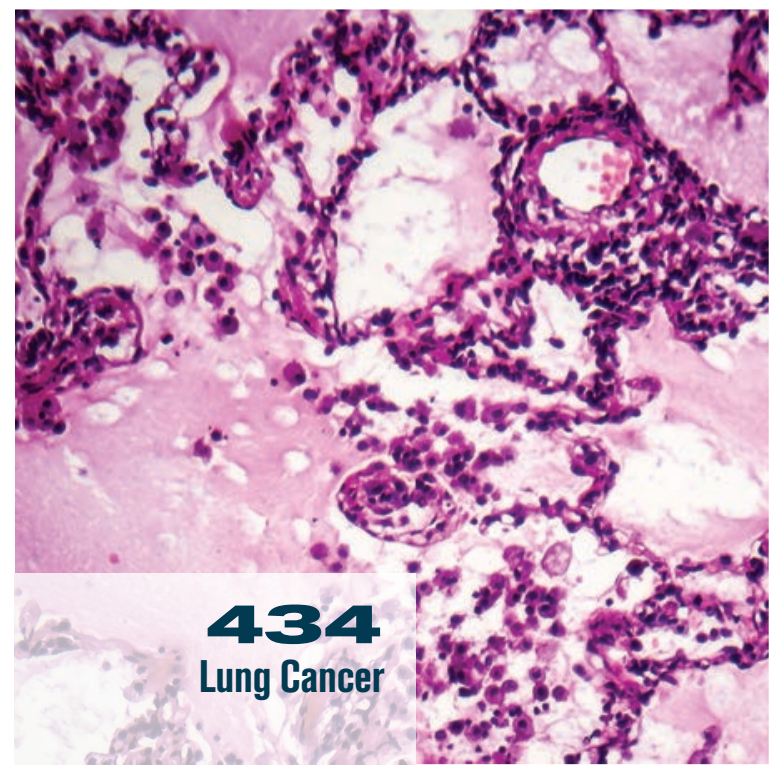

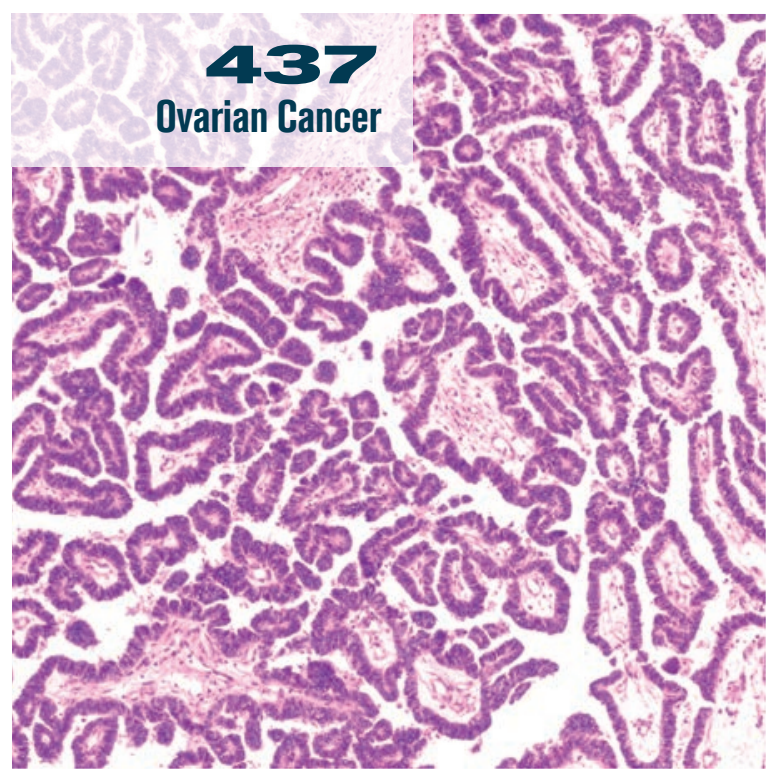




\section{Responses to Tafasitamab/Lenalidomide in DLBCL Are Sustained at 3 Years}

The combination of tafasitamab-cxix (Monjuvi) plus lenalidomide (Revlimid) for the treatment of relapsed or refractory diffuse large B-cell lymphoma (DLBCL) was capable of inducing sustained responses, according to 3-year follow-up data from the phase 2 L-MIND study (NCT02399085).

As of the data cutoff on October 30,2020, the best objective response rate (ORR) was $57.5 \%$ (95\% CI, $45.9 \%-68.5 \%)$ at a median follow-up of at least 35 months. Overall, $40 \%$ of patients experienced a complete response (CR) and $17.5 \%$ had a partial response. Median duration of response was 43.9 months (95\% CI, 26.1 to not reached). The best ORR was $67.5 \%$, with a CR rate of $47.5 \%$, in patients who received 1 prior treatment. The response rate was $47.5 \%$, with a $\mathrm{CR}$ rate of $32.5 \%$, in patients who received 2 or more prior treatments. The median progression-free survival and overall survival were 11.6 months and 33.5 months, respectively.

In this open-label, multinational, single-arm study, investigators assigned 81 patients with relapsed/refractory DLBCL to $12 \mathrm{mg} / \mathrm{kg}$ intravenous tafasitamab plus $25 \mathrm{mg}$ daily oral lenalidomide for up to twelve 28-day cycles. Patients with stable disease or better then received the same dose of tafasitamab, an Fc-modified humanized anti-CD19 monoclonal antibody, as monotherapy until disease progression.

Forty $(49.4 \%)$ patients had grade $\geq 3$ treatment-emergent neutropenia. Fourteen $(17.3 \%)$ had grade $\geq 3$ thrombocytopenia, while $10(12.3 \%)$ had grade $\geq 3$ febrile neutropenia.

$\rightarrow$ For the full article, visit cancernetwork.com/ASC021_L-Mind

\section{Patients With CLL and Other B-Cell Malignancies Benefit from TG-1701}

TG-1701 elicited promising clinical and pharmacodynamic activity across doses in patients with chronic lymphocytic leukemia (CLL) and other B-cell malignancies, according to updated results from a phase 1/2 trial (NCT03671590).

The maximum tolerated dose of the Bruton tyrosine kinase inhibitor was not reached in patients who received TG1701 monotherapy up to a dose of $400 \mathrm{mg}$ daily. The efficacy dose-escalation cohort, which received $100 \mathrm{mg}$ to $400 \mathrm{mg}$ of single-agent TG-1701 daily, achieved an overall response rate (ORR) of $57 \%$ after a median follow-up of 20.3 months. After a median follow-up of 12.2 months, the disease-specific monotherapy cohort, in which patients received $200 \mathrm{mg}$ of the agent daily, had an ORR of $95 \%$ in patients with CLL, $65 \%$ in those with mantle cell lymphoma, and $95 \%$ in those with Waldenström macroglobulinemia.

In the CLL cohort, a daily 300-mg monotherapy dose of TG1701 yielded an ORR of $100 \%$ at a median follow-up of 8.6 months. Additionally, a dose-escalation cohort that combined $100 \mathrm{mg}$ to $300 \mathrm{mg}$ of TG-1701 with ublituximab (TGTX1101) and umbralisib (Ukoniq) daily achieved an ORR of $79 \%$ at a median follow-up of 15.6 months across multiple B-cell malignancies, with a complete response rate of $21 \%$.

The dose-escalation portion of the trial included a TG-1701 monotherapy cohort and a second cohort evaluating the combination of TG-1701 plus ublituximab/umbralisib. After the optimal dose had been determined, the trial then moved on to focus on the disease-specific cohorts, which examined both the single-agent and combination regimens. Across most study cohorts, the most common reason for treatment discontinuation was disease progression. Two patients discontinued due to non-treatment-related adverse effects in the 200-mg disease-specific cohort.

$\rightarrow$ For the full article, visit cancernetwork.com/ASC021_TG-1701

\section{High Rate of Response Is Noted in MCL and CLL With Cirmtuzumab Plus Ibrutinib}

Patients with mantle cell lymphoma (MCL) or chronic lymphocytic leukemia (CLL) who were treated with cirmtuzumab and ibrutinib (Imbruvica) in a phase 1/2 trial (NCT03088878) showed promising responses with therapy, and the combination had acceptable tolerability.

The objective response rates for patients with MCL and CLL were $83.3 \%$ and $91.1 \%$, respectively. The complete response rates were $38.9 \%$ and $14.7 \%$ for the MCL and CLL arms, respectively. Ultimately, $94.4 \%$ of patients with MCL and $100 \%$ of patients with CLL derived a clinical benefit from the cirmtuzumab/ibrutinib regimen. The median progression-free survival was not reached in either arm.

Because MCL and CLL are considered incurable, the study aimed to test the efficacy and safety profile of cirmtuzum$\mathrm{ab}$-an agent that inhibits the tumor-promoting activity of oncoembryonic tyrosine kinase receptor ROR1, which is found in many solid and hematologic cancers-plus ibrutinib in patients with relapsed/refractory (R/R) MCL or treatment-naïve or R/R CLL.

The study was performed in 3 parts with separate arms: part 1, for dose escalation; part 2, for dose expansion; and part 3, comparing cirmtuzumab plus ibrutinib with ibrutinib alone in CLL. Overall, 26 patients with refractory MCL and 34 patients with treatment-naïve or R/R CLL were enrolled in the study. 
Adverse events with $20 \%$ or greater incidence included fatigue $(\mathrm{n}=11)$, diarrhea $(\mathrm{n}=9)$, contusion $(\mathrm{n}=7)$, dizziness ( $\mathrm{n}$ $=7)$, and nausea $(\mathrm{n}=7)$.

The phase 2 study for CLL is completed and is awaiting long-term follow-up. The phase 2 trial for MCL is currently enrolling.

$\rightarrow$ For the full article, visit cancernetwork.com/ASC021_cirmtuzumab

\section{Long-Term Clinical Benefit Is Observed With KTE-X19 for Treatment of Relapsed/Refractory B-ALL}

Heavily pretreated patients with relapsed/refractory B-cell acute lymphoblastic leukemia (B-ALL) who received a single infusion of a chimeric antigen receptor T-cell therapy, KTE-X19 (Tecartus), experienced robust and durable responses.

At a median follow-up of 16.4 months (range, 10.3-22.1) in the phase 2 ZUMA-3 trial (NCT02614066), the complete response/complete response with incomplete blood count recovery $(\mathrm{CR} / \mathrm{CRi})$ rate was $70.9 \%$, including a $\mathrm{CR}$ rate of $56.4 \%$. Additionally, $31 \%$ of responding patients were in ongoing remission at data cutoff, without the need for subsequent allogeneic stem cell transplantation (ASCT) consolidation. Moreover, the median overall survival was 18.2 months for all patients treated on the study and has not yet been reached in those who achieved a CR/CRi.

For patients who achieved a CR/CRi, the median time to CR/CRi was 1.1 months (range, 0.85-2.99). Additionally, 10 patients -9 who had achieved a CR/CRi and 1 with blast-free hypoplastic or aplastic bone marrow-underwent ASCT at a median of 98 days (range, 60-207) following treatment with KTE-X19. Additionally, the minimal residual disease negativity rate was $97 \%$ in responders.

The median duration of response was 12.8 months, with or without censoring patients at subsequent ASCT, and as of data cutoff, $31 \%$ of the patients who achieved a CR or CRi had ongoing remissions without receiving subsequent ASCT. Moreover, the median relapse-free survival was 11.6 months for all patients who received KTE-X19 and 14.2 months for those who achieved a CR/CRi.

In terms of safety, the most common grade ${ }^{3} 3$ adverse effects (AEs) were anemia (49\%) and pyrexia (36\%). There were also 10 instances of grade 5 AEs reported: 4 ALL; 2 treatment-emergent from KTE-X19 (brain herniation; septic shock); 3 occurring after initiation of another cancer treatment (fungal pneumonia; sepsis; respiratory failure); and 1 unrelated to treatment with KTE-X19 (pneumonia).

$\rightarrow$ For the full article, visit cancernetwork.com/ASC021_ZUMA-3

\section{Acalabrutinib Is Noninferior, Better Tolerated Than Ibrutinib in Previously Treated CLL}

Acalabrutinib (Calquence) was noninferior but better tolerated than ibrutinib (Imbruvica) in previously treated patients with chronic lymphocytic leukemia (CLL) with deletion $17 \mathrm{p}$ (del [17p]) or $\operatorname{del}(11 q)$.

Findings from an open-label, randomized, noninferiority phase 3 trial (NCT02477696) compared acalabrutinib and ibrutinib in 533 patients. Patients were randomized to receive 1 of the 2 Bruton tyrosine kinase inhibitors until disease progression or unacceptable toxicity. The primary end point was progression-free survival (PFS), and secondary end points were all-grade atrial fibrillation, grade 3 or higher infection, Richter transformation, and overall survival.

Overall, 268 patients received acalabrutinib and 265 received ibrutinib. Median age was 66 years and patients had a median of 2 prior lines of therapy; $45.2 \%$ of patients had $\operatorname{del}(17 \mathrm{p})$ and $64.2 \%$ had $\operatorname{del}(11 \mathrm{q})$.

At a median follow-up of 40.9 months, acalabrutinib was noninferior to ibrutinib, with a PFS of 38.4 months in both arms (HR, 1.00; 95\% CI, 0.79-1.27). Median overall survival was not reached in either arm. There were $63(23.5 \%)$ deaths in the acalabrutinib arm and $73(27.5 \%)$ in the ibrutinib arm.

Acalabrutinib was superior in all-grade atrial fibrillation incidence compared with ibrutinib $(9.4 \%$ vs $16.0 \%$, respectively; $P=.023$ ).

$\rightarrow$ For the full article, visit cancernetwork.com/ASC021_BTK

\section{Philippe Moreau, MD, on Daratumumab Maintenance for Newly Diagnosed Myeloma}

ONCOLOG $Y^{\circledR}$ spoke with Philippe Moreau, MD, of the University Hospital of Nantes, regarding implications from part 2 of the phase 3 CASSIOPEIA trial (NCT02541383), which investigated daratumumab (Darzalex) maintenance following consolidation with bortezomib, thalidomide, and dexamethasone (VTd) or daratumumab plus VTd (dara-VTd) in patients with newly diagnosed multiple myeloma who had received autologous stem cell transplant (ASCT).

Initial results of the trial compared the combination of daraVTd versus VTd alone as consolidation/induction in this patient population and led to the approval of dara-VTd for this indication. The current analysis looked at the addition of daratumumab maintenance for patients who had a response to therapy in part 1 of the trial.

"The main takeaway is that daratumumab maintenance is 
able to improve outcomes following stem cell transplantation, [although] this is restricted to patients who are not exposed as part of frontline treatment with a quadruplet [regimen] including daratumumab," Moreau said. "We definitely need a longer follow-up to look at progression-free survival 2 [PFS2] and overall survival. When we evaluated PFS2, we also showed that there is a clear separation of the curves that is now occurring with a follow-up of 3 years; we definitely need a longer follow-up. The best PFS2 curve is the one for patients who received daratumumab up front and daratumumab maintenance, as well.”

$\rightarrow$ To see the interview, visit cancernetwork.com/ASC021_Moreau

\section{CARTITUDE-2 Reports Strong Efficacy Data for Cilta-Cel in Multiple Myeloma}

Patients who had received 1 to 3 prior lines of therapy for multiple myeloma experienced early and deep responses when treated with a single infusion of ciltacabtagene autoleucel (cilta-cel), according to data from the phase 2 CARTITUDE-2 study (NCT04133636).

Treatment with a target dose of cilta-cel at $0.75 \times 10^{6} / \mathrm{kg}$ resulted in an overall response rate of $95 \%$ (95\% CI, 75\%$100 \%$ ) with a stringent complete response rate of $75 \%(95 \%$ CI, $51 \%-91 \%$ ), and a very good partial response rate or better of $85 \%$ (95\% CI, $62 \%-97 \%)$.

After a median follow-up of 5.8 months, the median time to first response with cilta-cel was 1.0 month (range, 0.7-3.3) and the median time to best response was 1.9 months (0.95.1). As of the January 2021 data cutoff, a median duration of response had not yet been reached.

Cilta-cel, formerly JNJ-68284528, is a second-generation chimeric antigen receptor T-cell therapy with 2 BCMA-targeting, single-domain antibodies designed to confer avidity.

The primary objective in studying this cohort of the CARTITUDE-2 trial was to assess minimal residual disease (MRD) negativity at the $10^{-5}$ threshold among patients who were refractory to lenalidomide (Revlimid) or had relapsed after 1 to 3 prior lines of therapy. All patients $(n=4)$ with MRD-evaluable samples were MRD negative at the January 2021 cutoff, and additional MRD-evaluable samples are being looked at.

$\rightarrow$ For the full article, visit cancernetwork.com/ASC021_CARTITUDE-2

\section{Compared With ASCT, KCd Consolidation Was Noninferior for Newly Diagnosed, Transplant- Eligible MM}

Compared with upfront autologous stem cell transplantation (ASCT) for newly diagnosed, transplant-eligible patients with multiple myeloma, carfilzomib (Kyprolis) consolidation with cyclophosphamide and dexamethasone $(\mathrm{KCd})$ produced promising results but failed to meet noninferirority, according to data from the CARDAMON study (NCT02315716).

Of note, the 2-year progression-free survival (PFS) was similar among the treatment arms with a small margin of difference.

Patients at prerandomization received 4 cycles of biweekly carfilzomib at $56 \mathrm{mg} / \mathrm{m}^{2}$ with $500 \mathrm{mg}$ of cyclophosphamide and $40 \mathrm{mg}$ of dexamethasone weekly. Patients who achieved at least a partial response to therapy proceeded to peripheral blood stem cell harvests and were then randomized 1:1—either to melphalan $200 \mathrm{mg} / \mathrm{m}^{2}$ plus ASCT, or to 4 cycles of $\mathrm{KCd}$ consolidation. The entire study population then received 18 cycles of maintenance carfilzomib at $56 \mathrm{mg} / \mathrm{m}^{2}$ weekly.

Among patients with standard-risk disease, the very good partial response rate after $\mathrm{KCd}$ induction $(\mathrm{n}=207)$ was $58.5 \%$, which increased to $79.5 \%$ after consolidation $\operatorname{KCd~}(\mathrm{n}=79)$ and $76.8 \%$ after ASCT $(n=85)$. The minimal residual diseasenegativity rate was $25 \%$ after induction, $37.1 \%$ after consolidation, and $48.1 \%$ after ASCT. The results were relatively similar in the high-risk patient population. In those individuals, the very good partial response rate after induction $(\mathrm{n}=52)$ was $59.6 \%, 77.3 \%$ after consolidation $(\mathrm{n}=22)$, and $89.5 \%$ after ASCT $(n=19)$. The minimal residual disease-negativity rate was $24 \%$ after induction, $31.6 \%$ after consolidation, and $66.7 \%$ after ASCT.

Survival outcomes with KCd consolidation did not meet the threshold for noninferiority compared with ASCT. At a median follow-up of 37.5 months, the 2-year PFS rate in the ASCT arm was $76 \%$ (95\% CI, 66\%-83\%) compared with 70\% (95\% CI, $60 \%-78 \%$ ) in the KCd consolidation arm. The median PFS was not reached (NR) in patients in the ASCT arm vs 3.4 years (95\% CI, 2.4-NR) in the consolidation group.

In terms of genetic risks, patients with $\mathrm{KCd}$ consolidation and standard-risk genetics achieved a median PFS of 5.1 years vs 1.7 years in high-risk patients (HR, 2.7; 95\% CI, 1.8-4.1; $P<$ .001). Moreover, among high-risk patients, the median PFS in the ASCT arm was 4.7 years (95\% CI, 3.3-NR) compared with 1.2 years in the consolidation arm (95\% CI, 0.7-NR).

$\rightarrow$ For the full article, visit cancernetwork.com/ASC021_KCd 


\section{Adding Nivolumab to Chemo Improves Outcomes in Resectable NSCLC}

The addition of neoadjuvant nivolumab (Opdivo) plus platinum-doublet chemotherapy significantly improved pathological complete response (pCR) rates and led to a greater depth of pathological response compared with chemotherapy alone in patients with resectable non-small cell lung cancer (NSCLC), according to results from the phase 3 CheckMate 816 study (NCT02998528).

Investigators randomized 358 patients in a 1:1 ratio to either nivolumab $360 \mathrm{mg}$ plus chemotherapy ( $\mathrm{n}=179$ ) or chemotherapy alone $(\mathrm{n}=179)$. Three cycles of therapy were planned in the neoadjuvant window. Surgery was performed within 6 weeks following the last dose of neoadjuvant treatment. After surgery, patients had the option to receive adjuvant chemotherapy or radiotherapy per local standard of care; no adjuvant immunotherapy was permitted.

Nivolumab plus chemotherapy elicited a pCR rate of $23 \%$ in patients with stage IIIA disease $(\mathrm{n}=113)$, compared with $1 \%$ in the chemotherapy arm $(n=115)$. Patients with stage IB NSCLC who received nivolumab $(\mathrm{n}=10)$ had a pCR rate of $40 \%$ vs $0 \%$ in the chemotherapy group $(n=8)$. Patients with stage IIA disease registered pCR rates of $23 \%$ and $3 \%$ in the nivolumab/chemotherapy $(\mathrm{n}=30)$ and chemotherapy $(\mathrm{n}=32)$ arms, respectively. Patients with stage IIB NSCLC experienced a pCR rate of $24 \%$ in the nivolumab cohort $(n=25)$ vs $9 \%$ for those treated with chemotherapy $(n=23)$.

No clinically meaningful differences were observed in time to surgery: Median time to definitive surgery was 5.3 weeks (range, 4.6-6.0) in the nivolumab plus chemotherapy arm and 5.0 weeks (range, 4.6-5.9) in the chemotherapy cohort. Surgical procedures were delayed or occurred beyond the 6-week protocol window for $21 \%$ of patients in the nivolumab/chemotherapy arm vs $18 \%$ for those in the chemotherapy group.

$\rightarrow$ For the full article, visit cancernetwork.com/ASC021_CheckMate816

\section{ARROW Trial Continues to Support Pralsetinib in RET Fusion+ NSCLC}

Durable responses coupled with a tolerable safety profile of pralsetinib (Gavreto) in patients with RET fusion-positive non-small cell lung cancer (NSCLC) were reported from the ARROW trial (NCT03037385), further supporting use of the agent in this patient subset.

Initial findings from the phase 1/2 trial led to the accelerated approval of the agent in the indicated patient population.
The phase $1 / 2$ study included 233 patients with RET fusionpositive NSCLC and 238 patients with other RET-altered solid tumors. Originally, patients who were treatment naive were not eligible, but the requirement was later removed by a protocol amendment.

Follow-up was conducted for a median of 17.1 months. For the 216 patients who received pralsetinib and were RET fusion positive, the objective response rate was $69 \%$ (95\% CI, 62\%$75 \%$ ), the disease control rate (DCR) was $92 \%$ (95\% CI, $87 \%$ $95 \%$ ), clinical benefit rate was 77\% (95\% CI, 71\%-82\%), and median duration of response (DOR) was 22.3 months (95\% CI, 15.1 to not reached [NR]). In the posteligibility population with 25 patients, the objective response rate was $88 \%$ (95\% CI, 69\%-98\%), CBR was $88 \%$ (95\% CI, $69 \%-98 \%$ ), and DCR was $96 \%$ (95\% CI, 80\%-100\%). In addition, median DOR and median PFS were NR. The median DOR in patients with prior platinum-based chemotherapy was 22.3 months (95\% CI, 15.1-NR).

Overall, $6 \%$ of patients in the entire study (26 of 471) discontinued treatment due to treatment-related adverse events.

$\rightarrow$ For the full article, visit cancernetwork.com/ASC021_ARROW

\section{EGFR ex 20+ NSCLC After Platinum Chemotherapy Responds to Mobocertinib Monotherapy}

Deep and durable responses to single-agent mobocertinib were observed in patients with metastatic non-small cell lung cancer (NSCLC) harboring EGFR exon 20 insertion mutations following prior treatment with platinum chemotherapy, according to data from a phase $1 / 2$ trial (NCT02716116).

In the platinum-pretreated patient cohort of the trial $(\mathrm{n}=$ 114 ), the confirmed overall response rate (ORR) by independent review committee (IRC) assessment was 28\% (95\% CI, 20\%-37\%); all responses were partial responses (PRs). By investigator assessment, the confirmed ORR was 35\% (95\% CI, $26 \%-45 \%$ ); the PR rate was $34 \%$, with a complete response rate of less than $1 \%$.

The median duration of response was 17.5 months (95\% CI, 7.4-20.3) by IRC and 11.2 months (95\% CI, 5.6 to not evaluable) by investigator assessment. The confirmed disease control rate was $78 \%$ (95\% CI, 69\%-85\%) by both IRC and investigator assessment.

The median overall survival was 24.0 months $(95 \% \mathrm{CI}$, 14.6-28.8) and the median progression-free survival was 7.3 months (95\% CI, 5.5-9.2).

$\rightarrow$ For the full article, visit cancernetwork.com/ASC021_mobocertinib 


\section{OS by Patient Subgroups Support Frontline Avelumab Maintenance in Advanced Urothelial Cancer}

In a post hoc analysis of the phase 3 JAVELIN Bladder 100 trial (NCT02603432), avelumab (Bavencio) as frontline maintenance was superior to best supportive care (BSC) alone across several previously unreported subgroups of patients with advanced urothelial cancer who had not progressed on first-line platinum-containing chemotherapy.

The progression-free survival (PFS) and overall survival (OS) improvements were seen in patients with upper tract or lower tract disease; metastatic disease, unresectable locally advanced disease, or lymph node-only disease; PD-L1-positive tumors who received frontline gemcitabine/carboplatin; and tumor genomic subtypes defined by The Cancer Genome Atlas, with the exception of the luminal subtype (HR, 1.01; 95\% CI, 0.403-2.509).

The PFS and OS benefits were observed irrespective of primary tumor site or type of advanced disease. While there was a greater difference of OS outcomes in those with lower tract primary tumors (HR, 0.62; 95\% CI, 0.477-0.802), unresectable locally advanced disease (HR, 0.40; 95\% CI, 0.265-0.617), or lymph node-only disease following chemotherapy (HR, $0.55 ; 95 \% \mathrm{CI}, 0.259-1.152$ ), the investigators noted that the number of patients and events were small.

In the luminal-infiltrated group, the median OS was 19.9 months (95\% CI, 18.2 to not estimable [NE]) with avelumab/ BSC $(\mathrm{n}=143)$ and 14.3 months (95\% CI, 12.8-18.6) with BSC alone ( $\mathrm{n}=143$; HR, 0.68; 95\% CI, 0.481-0.968). Finally, in the luminal papillary subgroup, the median OS was 22.5 months (95\% CI, 18.2-26.0) with avelumab/BSC $(\mathrm{n}=61)$ compared with 13.4 months (95\% CI, 10.1-NE) with BSC alone ( $\mathrm{n}=63$; HR, 0.63; 95\% CI, 0.370-1.079).

Further data showed that longer outcomes were seen in the avelumab arm in patients with PD-L1-positive disease who received frontline gemcitabine/carboplatin for PFS (HR, 0.61; 95\% CI, 0.390-0.958) and OS (HR, 0.67; 95\% CI, 0.3931.137). These data were consistent with those of the overall study population.

$\rightarrow$ For the full article, visit cancernetwork.com/ASCO21_JAVELIN

\section{TURBT With Nivolumab/Chemo as Bladder- Sparing Strategy in MIBC}

As a bladder-sparing treatment strategy, transurethral resection of the bladder tumor (TURBT) with nivolumab (Opdivo) and chemotherapy showed promise for patients with muscle-in- vasive bladder cancer (MIBC), according to findings from the phase 2 HCRN GU16-257 trial (NCT03558087).

The study enrolled 76 patients with cisplatin-eligible cT2 to T4aN0M0 clinically localized urothelial bladder cancer. Tumor stages at baseline were cT2 (57\%), cT3 (32\%), and cT4 $(12 \%)$. Regarding histology, $76 \%$ of patients had transitional cell urothelial cancer and $24 \%$ had urothelial cancer with variant histology.

Patients initially received 4 cycles of gemcitabine plus cisplatin plus nivolumab. Following these 4 cycles, all patients underwent clinical restaging, including MRI/CT of the bladder (unless otherwise contraindicated), urine cytology, and cystoscopy with bladder/prostatic urethral biopsies. Patients with a clinical complete response (CR) could then opt to proceed with cystectomy or proceed without cystectomy and instead receive an additional 4 months of single-agent nivolumab. For patients without a clinical CR, a cystectomy was recommended.

At the time of the data cutoff, 64 patients had completed clinical restaging. Of these patients, 31 (48\%; 95\% CI, 36\%$61 \%$ ) achieved a clinical CR. Thirty of these patients chose not to undergo cystectomy and instead receive an additional 4 months of nivolumab monotherapy. Only 1 patient opted for immediate cystectomy.

Six patients with a clinical CR underwent delayed cystectomy after local recurrence. Of these patients, 1 patient each had a pathological stage of ypT0N0, ypTaN0, YpTisN0, and ypT4N1, and 2 patients had a stage of ypT2N0.

Among 28 patients who did not reach a clinical CR and underwent immediate cystectomy, the pathological stages were ypT0N0 ( $=2)$, YpTisN0 ( $=5), y p T 1 N 0(n=3), y p T 2 N 0$ ( $=5), \operatorname{ypT} 3 \mathrm{~N} 0(\mathrm{n}=3), \operatorname{ypT} 4 \mathrm{~N} 0(\mathrm{n}=1)$, and ypTanyN+ $(\mathrm{n}=9)$.

$\rightarrow$ For the full article, visit cancernetwork.com/ASC021_TURBT

\section{Abiraterone/ADT and Docetaxel in de novo mCSPC Improves rPFS}

For treating men with de novo metastatic castration-sensitive prostate cancer (mCSPC), adding abiraterone acetate (Zytiga) and prednisone to androgen-deprivation therapy (ADT) and docetaxel improved radiographic progression-free survival (rPFS).

The phase 3 PEACE-1 study (NCT01957436) randomized men with de novo mCSPC in a 1:1:1:1 ratio to standard of care (SOC), SOC plus abiraterone acetate/prednisone, SOC plus radiotherapy (RT), or SOC plus abiraterone plus RT. The findings showed that adding abiraterone/prednisone to standard docetaxel/ADT was associated with an approximately 2.5-year absolute benefit in median rPFS in this patient population.

The investigators found no interaction between the effect of 
local RT and that of abiraterone on rPFS, which allowed the pooling of the 2 abiraterone arms for analysis. When divided into abiraterone and SOC arms, patient characteristics were similar among the arms.

The investigators reported a median rPFS of 4.5 years in the abiraterone arm in the overall population vs 2.2 years for SOC $(P<.0001)$. Similarly, better rPFS was observed in the abiraterone arm vs the SOC arm in the ADT plus docetaxel population (4.5 vs 2.0 years; $P<.0001$ ). In addition, PFS including PSA progression as an event was also improved with abiraterone in both the overall population (3.8 vs 1.5 years; $P<.0001)$ and the ADT plus docetaxel population $(3.2$ vs 1.4 years; $P<.0001$ ).

In the docetaxel population, grade 3 to 5 toxicities were higher in patients receiving abiraterone and docetaxel vs patients receiving docetaxel alone. Grade 3 to 5 gastrointestinal toxicity and fatigue had a lower incidence in the group of patients receiving abiraterone ( $2 \%$ vs $4 \%$, respectively), which may be due to the abiraterone cohort also receiving prednisone.

$\rightarrow$ For the full article, visit cancernetwork.com/ASC021_PEACE-1

\section{HRQOL Analysis From CLEAR Further Supports Pembrolizumab/Lenvatinib for Frontline RCC}

The combination of lenvatinib (Lenvima) plus pembrolizumab (Keytruda) for the frontline treatment of metastatic renal cell carcinoma (RCC) led to similar health-related quality of life (HRQOL) outcomes and disease-related symptom scores vs sunitinib (Sutent) for patients treated in the phase 3 CLEAR trial (NCT02811861).

Looking at pembrolizumab/lenvatinib vs sunitinib, least square mean differences indicated similar to better HRQOL with the experimental regimen across different European Organisation for Research and Treatment of Cancer Quality of Life Questionnaire for Patients with Cancer-Core 30 (EORTC QLQ-C30) global health scale/QOL functional and symptom scales. Physical functioning (HR, 3.01; 95\% CI, 0.48-5.54; $P<.05$ ), fatigue (HR, $-2.80 ; 95 \%$ CI, -5.52 to $-0.08 ; P<.05)$, dyspnea (HR, $-2.79 ; 95 \% \mathrm{CI},-5.33$ to $-0.25 ; P<.05)$, and constipation (HR, $-2.19 ; 95 \% \mathrm{CI},-4.19$ to $-0.18 ; P<.05)$ all favored the combination.

Time to first deterioration by EORTC QLQ-C30 also favored the immune checkpoint inhibitor/tyrosine kinase inhibitor combination over sunitinib for physical functioning, with a median duration of 15.29 weeks vs 12.71 weeks, respectively (HR, 0.81; 95\% CI, 0.68-0.98; P = .03). Similarly, the median duration to onset was 39.29 weeks vs 21.14 weeks, respectively, for dyspnea (HR, 0.79; 95\% CI, 0.64-0.97; P = .02), and it was 18.29 weeks vs 9.14 weeks for appetite loss (HR, 0.82;
95\% CI, 0.68-0.98; $P=.03)$. Utilizing the European Organisation for Research and Treatment of Cancer Quality of Life Questionnaire for Patients with Cancer-Core 30 (EQ-5D-3L), the EQ-visual analogue scale similarly favored pembrolizumab plus lenvatinib, at 9.43 weeks vs 9.14 weeks (HR, 0.83; $95 \%$ CI, 0.70-0.99; $P=.04$ ).

Across all 3 scales, time to definitive deterioration was significantly improved with the combination of pembrolizumab and lenvatinib vs sunitinib.

$\rightarrow$ For the full article, visit cancernetwork.com/ASC021_CLEARHRQOL

\section{Long-Term Survival Benefit Is Reported for Pembrolizumab/Axitinib in RCC}

With 3.5 years of follow-up to the phase 3 KEYNOTE-426 trial (NCT02853331), frontline treatment of renal cell carcinoma (RCC) with the combination of pembrolizumab (Keytruda) plus axitinib (Inlyta) led to a survival benefit over sunitinib (Sutent).

The international, open-label study included 861 patients 18 years or older with treatment-naïve, advanced RCC. All patients had clear cell histology. Patients were randomized between October 24, 2016, and January 24, 2018, to either pembrolizumab plus axitinib $(n=432)$ or single-agent sunitinib ( $\mathrm{n}=429)$. In the combination arm, patients received pembrolizumab at $200 \mathrm{mg}$ intravenously every 3 weeks for up to 35 cycles plus axitinib at $5 \mathrm{mg}$ orally twice daily. In the control arm, patients were treated with sunitinib at $50 \mathrm{mg}$ orally once daily for the first 4 weeks of each 6-week cycle. Treatment was administered until disease progression, unacceptable toxicity, or patient withdrawal from the trial.

At a median follow-up of 42.8 months, the median overall survival was 45.7 months ( $95 \%$ CI, 43.6 to not reached) with pembrolizumab/axitinib compared with 40.1 months (95\% CI, 34.3-44.2) with sunitinib (HR, 0.73; 95\% CI, 0.60$0.88 ; P<.001)$. The median progression-free survival was 15.7 months (95\% CI, 13.6-20.2) vs 11.1 months (95\% CI, 8.912.5), respectively (HR, 0.68; 95\% CI, 0.58-0.80; $P<.0001$ ).

Objective response rate at 42.8 months was $60.4 \%$ in the combination arm compared with $39.6 \%$ in the sunitinib arm. The complete response and partial response rates were $10.0 \%$ vs $3.5 \%$ and $50.5 \%$ vs $36.1 \%$, respectively. The median time to response was 2.8 months with the combination vs 3.0 months with sunitinib. The median duration of response was 23.6 months vs 15.3 months, respectively.

The rates of grade 3 to 5 treatment-related adverse events were $67.8 \%$ vs $63.8 \%$, respectively.

$\rightarrow$ For the full article, visit cancernetwork.com/ASC021_KEYN0TE426 


\section{Multitrial Analysis Indicates Niraparib Benefit for BRCA+ Ovarian Cancer}

The compiled results from three phase 3 trials of niraparib (Zejula) maintenance in patients with BRCA-mutant ovarian cancer following platinum response in both front- and later-line settings indicate significant reduction in the risk of disease progression or death with the PARP inhibitor.

Researchers assessed the efficacy and safety of niraparib compared with placebo in patients with $B R C A$-mutated ovarian cancer from 3 trials: ENGOT-OV16/NOVA (NCT01847274; $\mathrm{n}=203$ ),PRIMA/ENGOT-OV26/GOG-3012 (NCT02655016; $\mathrm{n}=223$ ), and NORA (NCT03705156; $\mathrm{n}=100$ ).

Patients in the PRIMA trial had newly diagnosed advanced ovarian cancer and responded to first-line platinum-based chemotherapy, and patients in the ENGOT-OV16/NOVA and NORA trials had platinum-sensitive, recurrent ovarian cancer. All trials included a subgroup analysis by $B R C A$ mutation status.

Of the 526 patients in all 3 trials, the most common mutation was in BRCA1, accounting for $60.6 \%$ to $80.0 \%$ of $B R C A$ mutations.

Niraparib led to a significant progression-free survival (PFS) benefit compared with placebo in patients with $B R C A$ mutations from the PRIMA (HR, 0.4; 95\% CI, 0.27-0.62), NOVA (HR, 0.27 ; $95 \%$ CI, 0.17-0.41), and NORA trials (HR, 0.22; 95\% CI, 0.12-0.39). No differences were observed in the PRIMA trial between patients with $B R C A 1$ and $B R C A 2$ mutations. For patients in the NOVA trial, hazard ratios for PFS were 0.39 for those with BRCA1 mutations (95\% CI, 0.23-0.66) and 0.12 for those with BRCA2 mutations (95\% CI, 0.05-0.33).

$\rightarrow$ For the full article, visit cancernetwork.com/ASC021_niraparib

\section{No Survival Difference Is Noted With Longer Bevacizumab Duration in Ovarian Cancer}

For patients with primary epithelial ovarian, fallopian tube, or peritoneal cancer, prolonged treatment of up to 30 months with bevacizumab (Avastin) is feasible and safe, although not more efficacious in terms of an overall survival improvement, according to results from the phase 3 AGO-OVAR 17/BOOST/ GINECO OV118/ENGOT Ov-15 trial (NCT01462890).

From November 2011 to August 2013, 927 patients were enrolled on the study and randomized 1:1 to receive either bevacizumab for 30 months $(n=463)$ or for 15 months $(n=$ 464). Patients were stratified based on FIGO stage IIB to stage IIIC disease with no residual tumor, FIGO stage IIB or stage IIIC disease with residual tumor, or FIGO stage IV disease.

The median progression-free survival (PFS) for patients treated with bevacizumab for 30 months was 26.0 months (95\% CI, 23.7-29.7) vs 24.2 months (95\% CI, 22.2-26.5) for those treated for 15 months (HR, 0.99; 95\% CI, 0.85-1.15), which was not significantly different $(P=.90)$. Additionally, due to evidence suggesting nonproportional distribution of events, a restricted mean analysis was performed, which demonstrated a mean PFS of 39.3 months (95\% CI, 36.2-42.4) in the experimental arm compared with 39.5 months $(95 \%$ CI, 36.3-42.7) in the standard-of-care arm, which was also not statistically significantly different $(P=.92)$.

The median overall survival for the experimental arm was 60.0 months (95\% CI, 54.0-68.6) vs 54.3 months (95\% CI, 51.0-64.6) for the standard arm (HR, 1.04; 95\% CI, 0.871.23; $P=.68)$. Moreover, the corresponding restricted OS means were 60.8 months (95\% CI, 57.8-63.8) and 60.4 months (95\% CI, 57.2-63.6) $(P=.97)$.

Additionally, due to a low event rate, the study was closed after $97 \%$ of the planned events $(n=673 / 697)$ had been observed. Moreover, at closure, the conditional probability of reaching a significant result with the full event number was $7.5 \%$.

$\rightarrow$ For the full article, visit cancernetwork.com/ASC021_AG0-0VAR17

\section{Platinum-Agnostic Recurrent Ovarian Cancer May Benefit From Mirvetuximab Soravtansine Plus Bevacizumab}

Response rates have shown promise for the combination of mirvetuximab soravtansine (IMGN853) and bevacizumab (Avastin) in treating patients with platinum-agnostic ovarian cancer in the recurrent disease setting, according to data from the phase 1b FORWARD II trial (NCT02606305).

Trial results indicate that the total patient population $(\mathrm{n}=$ 60 ) achieved an overall response rate (ORR) of $50 \%$. Moreover, patients who had high folate receptor alpha (FR $\alpha ; n=$ 33 ) expression had an ORR of $64 \%$ vs $33 \%$ in patients with medium levels of FR $\alpha$ expression $(n=27)$. Patients with platinum-resistant ovarian cancer $(\mathrm{n}=17)$ had an ORR of $59 \%$, while patients with platinum-sensitive disease $(n=16)$ had an ORR of $69 \%$. Additionally, $97 \%$ of patients demonstrated a reduction in tumor burden following treatment.

The median duration of response (DOR) across all patients was 9.7 months, while those with medium and high FR $\alpha$ expression had median DORs of 8.3 months and 11.8 months, respectively. Additionally, patients with platinum-resistant disease had a median DOR of 9.4 months, and the platinumsensitive subgroup had a median DOR of 12.7 months.

$\rightarrow$ For the full article, visit cancernetwork.com/ASC021_mirvetuximab 


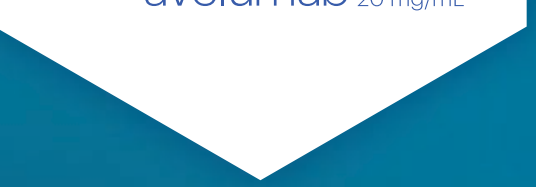

First-line maintenance treatment of urothelial carcinoma

$\mathrm{BAVENCIO}^{\circledR}$ (avelumab) is indicated for the maintenance treatment of patients with locally advanced or metastatic urothelial carcinoma (UC) that has not progressed with first-line platinum-containing chemotherapy.

\section{Based on overall survival (OS) data The FIRST and ONLY immunotherapy approved in the first-line maintenance setting}

NCCN

CATEGORY 1 \& PREFERRED

\section{National Comprehensive Cancer Network ${ }^{\circledast}$ NCCN $\left.^{\circledast}\right)$}

Recommendation

Avelumab (BAVENCIO) maintenance is the only NCCN CATEGORY 1 and PREFERRED immunotherapy

option for both cisplatin-eligible and -ineligible patients

with locally advanced or metastatic UC that has not progressed on

first-line platinum-containing chemotherapy. ${ }^{1}$

Category 1 =Based upon high-level evidence, there is uniform NCCN consensus that the intervention is appropriate.

Preferred intervention=Interventions that are based on superior efficacy, safety, and evidence; and, when appropriate, affordability.

\section{IMPORTANT SAFETY INFORMATION (continues on following pages)}

BAVENCIO can cause severe and fatal immune-mediated adverse reactions in any organ system or tissue and at any time after starting treatment with a PD-1/PD-L1 blocking antibody, including after discontinuation of treatment.

Early identification and management of immune-mediated adverse reactions are essential to ensure safe use of PD-1/PD-L1 blocking antibodies. Monitor patients closely for symptoms and signs that may be clinical manifestations of underlying immune-mediated adverse reactions. Evaluate liver enzymes, creatinine, and thyroid function at baseline and periodically during treatment. In cases of suspected immune-mediated adverse reactions, initiate appropriate workup to exclude alternative etiologies, including infection. Institute medical management promptly, including specialty consultation as appropriate. No dose reduction for BAVENCIO is recommended. For immunemediated adverse reactions, withhold or permanently discontinue BAVENCIO depending on severity. In general, withhold BAVENCIO for severe (Grade 3) immune-mediated adverse reactions. Permanently discontinue BAVENCIO for life-threatening (Grade 4) immune-mediated adverse reactions, recurrent severe (Grade 3 ) immune-mediated reactions that require systemic immunosuppressive treatment, or an inability to reduce corticosteroid dose to $10 \mathrm{mg}$ or less of prednisone or equivalent per day within 12 weeks of initiating corticosteroids. In general, if BAVENCIO requires interruption or discontinuation, administer systemic corticosteroid therapy ( 1 to $2 \mathrm{mg} / \mathrm{kg} /$ day prednisone or equivalent) until improvement to Grade 1 or less. Upon improvement to Grade 1 or less, initiate corticosteroid taper and continue to taper over at least 1 month. Consider administration of other systemic immunosuppressants in patients whose immunemediated adverse reactions are not controlled with corticosteroid therapy. Toxicity management guidelines for adverse reactions that do not necessarily require systemic corticosteroids (eg, endocrinopathies and dermatologic reactions) are discussed in subsequent sections.

BAVENCIO can cause immune-mediated pneumonitis. Withhold BAVENCIO for Grade 2, and permanently discontinue for Grade 3 or Grade 4 pneumonitis. Immune-mediated pneumonitis occurred in $1.2 \%(21 / 1738)$ of patients, including fatal $(0.1 \%)$, Grade $4(0.1 \%)$, Grade $3(0.3 \%)$, and Grade $2(0.6 \%)$ adverse reactions. Systemic corticosteroids were required in all $(21 / 21)$ patients with pneumonitis.

BAVENCIO can cause immune-mediated colitis. The primary component of immune-mediated colitis consisted of diarrhea. Cytomegalovirus infection/reactivation has been reported in patients with corticosteroid-refractory immune-mediated colitis. In cases of corticosteroid-refractory colitis, consider repeating infectious workup to exclude alternative etiologies. Withhold BAVENCIO for Grade 2 or Grade 3, and permanently discontinue for Grade 4 colitis. Immunemediated colitis occurred in $1.5 \%(26 / 1738)$ of patients, including Grade $3(0.4 \%)$ and Grade $2(0.7 \%)$ adverse reactions. Systemic corticosteroids were required in all $(26 / 26)$ patients with colitis. 
JAVELIN Bladder 100 Trial-a Phase 3, randomized, open-label, multicenter study in patients with unresectable, locally advanced or metastatic urothelial carcinoma that did not progress with first-line platinum-containing chemotherapy $(\mathrm{N}=700)^{2}$

\section{BAVENCIO $^{\circledR}$ (avelumab) + best supportive care (BSC) demonstrated superior OS vs BSC alone}

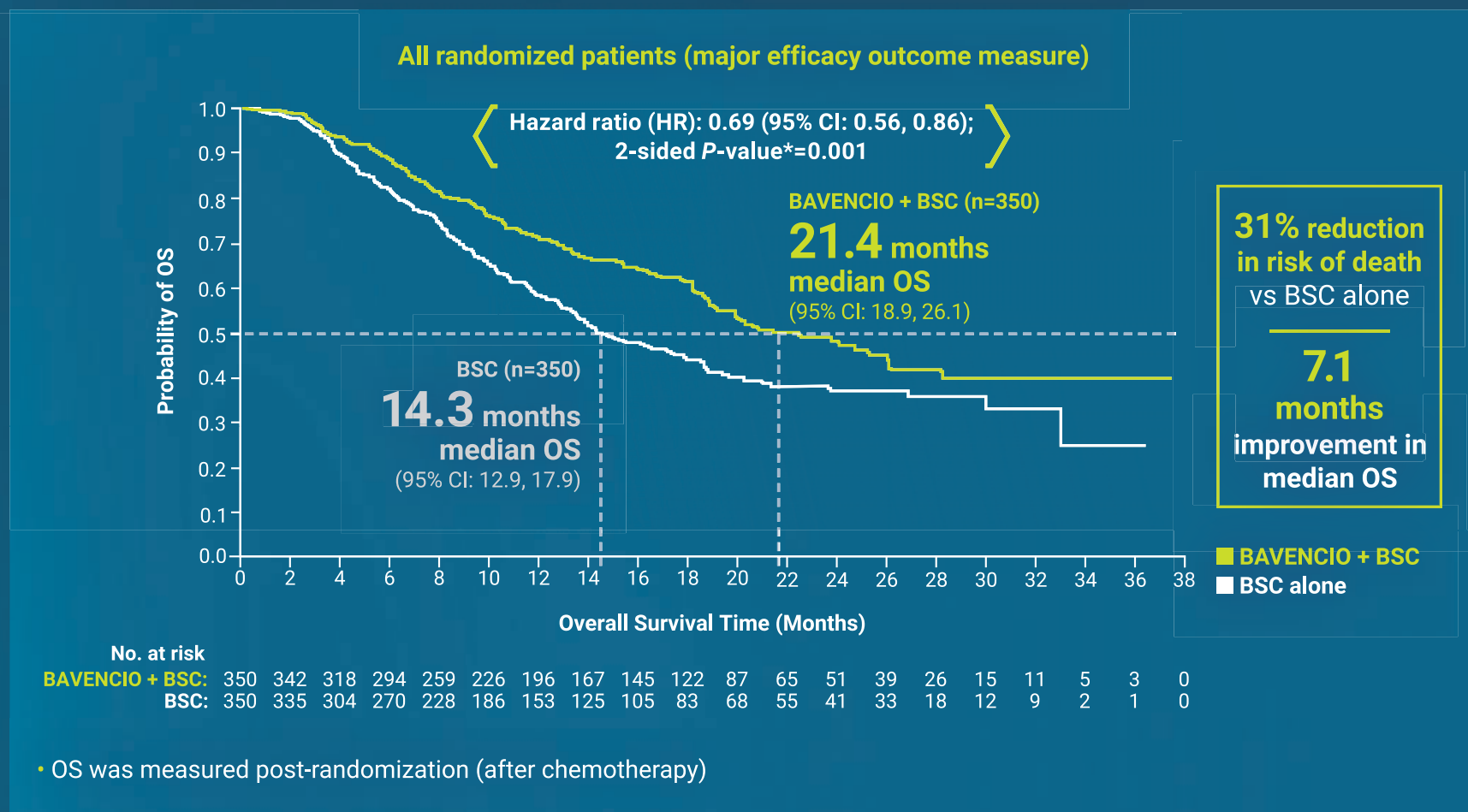

- OS in patients with PD-L1-positive tumors ${ }^{\dagger}$ (major efficacy outcome measure). BAVENCIO + BSC showed statistically significant improvement in OS vs BSC alone in patients with PD-L1-positive tumors ( $\mathrm{n}=358,51 \%)$; HR: 0.56; $(95 \%$ Cl: 0.40, 0.79; 2-sided P-value $<0.001)$

- OS in patients with PD-L1-negative tumors ${ }^{\dagger}$ (exploratory analysis). In patients with PD-L1-negative tumors ( $\left.\mathrm{n}=271,39 \%\right)$, the 0 S hazard ratio was $0.85(95 \% \mathrm{Cl}: 0.62,1.18)$

Most common adverse reactions in the JAVELIN Bladder 100 Trial

The most common adverse reactions $(\geq 20 \%)$ in patients receiving BAVENCIO + BSC vs BSC alone were:
- Fatigue (35\% vs $13 \%)$
- Musculoskeletal pain (24\% vs $15 \%)$
- Urinary tract infection ( $20 \%$ vs $11 \%)$
- Rash (20\% vs $2.3 \%)$

For information on warnings and precautions, see Important Safety Information starting on the previous page.

Study design: The JAVELIN Bladder 100 Trial was a Phase 3, 1:1 randomized, open-label, multicenter study of BAVENCIO as a first-line maintenance treatment in 700 patients with unresectable, locally advanced or metastatic UC who did not progress on 4 to 6 cycles of platinum-containing chemotherapy (gemcitabine + cisplatin and/or gemcitabine + carboplatin), and an ECOG PS of 0 or 1. ${ }^{2}$ Patients with autoimmune diseases or medical conditions requiring systemic immunosuppression were excluded. Patients were randomized to BAVENCIO $10 \mathrm{mg} / \mathrm{kg}$ intravenous infusion every 2 weeks + best supportive care $(B S C)(n=350)$ or $B S C$ alone ${ }^{\ddagger}(n=350)$ until disease progression or unacceptable toxicity. Treatment was initiated within 4 to 10 weeks after chemotherapy. OS was the major efficacy outcome measure in all randomized patients and patients with PD-L1-positive tumors. ${ }^{\S}$

\footnotetext{
*P-value based on stratified log-rank. approaches with palliative intent (excludes systemic antitumor therapy). ${ }^{2}$

§PD-L1 expression was assessed in tumor samples using the VENTANA PD-L1 (SP263) assay. ${ }^{2}$

BICR=blinded independent central review; $\mathrm{Cl}=$ confidence interval:

ECOG PS=Eastern Cooperative Oncology Group (ECOG) Performance Status;

PD-1=programmed death-1 receptor; PD-L1=programmed death ligand-1.
}

+ Using the VENTANA PD-L1 (SP263) assay, PD-L1-positive status was defined as PD-L1 expression in $\geq 25 \%$ of tumor cells or in $\geq 25 \%$ or $100 \%$ of tumor-associated immune cells if the percentage of immune cells was $>1 \%$ or $\leq 1 \%$, respectively. If none of these criteria were met, PD-L1 status was considered negative. ${ }^{2}$

‡BSC was administered as deemed appropriate by the treating physician, and could include treatment with antibiotics, nutritional support, and other patient management

Please see additional Important Safety Information and Brief Summary of the Prescribing Information on the following pages. 
BAVENCIO ${ }^{\circledR}$ (avelumab) can cause hepatotoxicity and immunemediated hepatitis. Withhold or permanently discontinue BAVENCIO based on tumor involvement of the liver and severity of aspartate aminotransferase (AST), alanine aminotransferase (ALT), or total bilirubin elevation. Immune-mediated hepatitis occurred with BAVENCIO as a single agent in $0.9 \%(16 / 1738)$ of patients, including fatal $(0.1 \%)$, Grade $3(0.6 \%)$, and Grade $2(0.1 \%)$ adverse reactions. Systemic corticosteroids were required in all $(16 / 16)$ patients with hepatitis.

BAVENCIO can cause primary or secondary immune-mediated adrenal insufficiency. For Grade 2 or higher adrenal insufficiency, initiate symptomatic treatment, including hormone replacement, as clinically indicated. Withhold BAVENCIO for Grade 3 or Grade 4 endocrinopathies until clinically stable or permanently discontinue depending on severity. Immune-mediated adrenal insufficiency occurred in $0.5 \%(8 / 1738)$ of patients, including Grade $3(0.1 \%)$ and Grade $2(0.3 \%)$ adverse reactions. Systemic corticosteroids were required in all $(8 / 8)$ patients with adrenal insufficiency.

BAVENCIO can cause immune-mediated hypophysitis. Hypophysitis can present with acute symptoms associated with mass effect such as headache, photophobia, or visual field defects. Hypophysitis can cause hypopituitarism. Initiate hormone replacement, as clinically indicated. Withhold BAVENCIO for Grade 3 or Grade 4 endocrinopathies until clinically stable or permanently discontinue depending on severity. Immune-mediated pituitary disorders occurred in $0.1 \%(1 / 1738)$ of patients, which was a Grade $2(0.1 \%)$ adverse reaction.

BAVENCIO can cause immune-mediated thyroid disorders. Thyroiditis can present with or without endocrinopathy. Hypothyroidism can follow hyperthyroidism. Initiate hormone replacement for hypothyroidism or institute medical management of hyperthyroidism, as clinically indicated. Withhold BAVENCIO for Grade 3 or Grade 4 endocrinopathies until clinically stable or permanently discontinue depending on severity. Thyroiditis occurred in $0.2 \%$ (4/1738) of patients, including Grade $2(0.1 \%)$ adverse reactions. Hyperthyroidism occurred in $0.4 \%(7 / 1738)$ of patients, including Grade $2(0.3 \%)$ adverse reactions. Systemic corticosteroids were required in $29 \%$ (2/7) of patients with hyperthyroidism. Hypothyroidism occurred in $5 \%(90 / 1738)$ of patients, including Grade $3(0.2 \%)$ and Grade $2(3.7 \%)$ adverse reactions. Systemic corticosteroids were required in $7 \%(6 / 90)$ of patients with hypothyroidism.

BAVENCIO can cause immune-mediated type I diabetes mellitus, which can present with diabetic ketoacidosis. Monitor patients for hyperglycemia or other signs and symptoms of diabetes. Initiate treatment with insulin as clinically indicated. Withhold BAVENCIO for Grade 3 or Grade 4 endocrinopathies until clinically stable or permanently discontinue depending on severity. Immune-mediated type I diabetes mellitus occurred in $0.1 \%(2 / 1738)$ of patients, including Grade $3(0.1 \%)$ adverse reactions.

BAVENCIO can cause immune-mediated nephritis with renal dysfunction. Withhold BAVENCIO for Grade 2 or Grade 3, and permanently discontinue for Grade 4 increased blood creatinine. Immune-mediated nephritis with renal dysfunction occurred in $0.1 \%$ $(1 / 1738)$ of patients, which was a Grade $2(0.1 \%)$ adverse reaction. Systemic corticosteroids were required in this patient.

BAVENCIO can cause immune-mediated dermatologic adverse reactions, including rash or dermatitis. Exfoliative dermatitis including Stevens Johnson Syndrome (SJS), drug rash with eosinophilia and systemic symptoms (DRESS), and toxic epidermal necrolysis (TEN), has occurred with PD-1/PD-L1 blocking antibodies. Topical emollients and/or topical corticosteroids may be adequate to treat mild to moderate non-exfoliative rashes. Withhold BAVENCIO for suspected and permanently discontinue for confirmed SJS, TEN, or DRESS. Immune-mediated dermatologic adverse reactions occurred in 5\% (90/1738) of patients, including Grade $3(0.1 \%)$ and Grade $2(2.0 \%)$ adverse reactions. Systemic corticosteroids were required in $29 \%$ $(26 / 90)$ of patients with dermatologic adverse reactions.
BAVENCIO can result in other immune-mediated adverse reactions. Other clinically significant immune-mediated adverse reactions occurred at an incidence of $<1 \%$ in patients who received BAVENCIO or were reported with the use of other PD-1/PD-L1 blocking antibodies. For myocarditis, permanently discontinue BAVENCIO for Grade 2, Grade 3, or Grade 4. For neurological toxicities, withhold BAVENCIO for Grade 2 and permanently discontinue for Grade 3 or Grade 4.

BAVENCIO can cause severe or life-threatening infusion-related reactions. Premedicate patients with an antihistamine and acetaminophen prior to the first 4 infusions and for subsequent infusions based upon clinical judgment and presence/severity of prior infusion reactions. Monitor patients for signs and symptoms of infusion-related reactions, including pyrexia, chills, flushing, hypotension, dyspnea, wheezing, back pain, abdominal pain, and urticaria. Interrupt or slow the rate of infusion for Grade 1 or Grade 2 infusion-related reactions. Permanently discontinue BAVENCIO for Grade 3 or Grade 4 infusion-related reactions. Infusion-related reactions occurred in $25 \%$ of patients, including three $(0.2 \%)$ Grade 4 and nine $(0.5 \%)$ Grade 3 infusion-related reactions. Eleven (92\%) of the 12 patients with Grade $\geq 3$ reactions were treated with intravenous corticosteroids.

Fatal and other serious complications of allogeneic hematopoietic stem cell transplantation (HSCT) can occur in patients who receive HSCT before or after being treated with a PD-1/PD-L1 blocking antibody. Follow patients closely for evidence of transplant-related complications and intervene promptly. Consider the benefit versus risks of treatment with a PD-1/PD-L1 blocking antibody prior to or after an allogeneic HSCT.

BAVENCIO can cause fetal harm when administered to a pregnant woman. Advise patients of the potential risk to a fetus including the risk of fetal death. Advise females of childbearing potential to use effective contraception during treatment with BAVENCIO and for at least 1 month after the last dose of BAVENCIO. It is not known whether BAVENCIO is excreted in human milk. Advise a lactating woman not to breastfeed during treatment and for at least 1 month after the last dose of BAVENCIO due to the potential for serious adverse reactions in breastfed infants.

A fatal adverse reaction (sepsis) occurred in one (0.3\%) patient with locally advanced or metastatic urothelial carcinoma (UC) receiving BAVENCIO + best supportive care (BSC) as first-line maintenance treatment. In patients with previously treated locally advanced or metastatic UC, fourteen patients $(6 \%)$ who were treated with BAVENCIO experienced either pneumonitis, respiratory failure, sepsis/urosepsis, cerebrovascular accident, or gastrointestinal adverse events, which led to death.

The most common adverse reactions (all grades, $\geq 20 \%$ ) in patients with locally advanced or metastatic UC receiving BAVENCIO + BSC (vs BSC alone) as first-line maintenance treatment were fatigue (35\% vs $13 \%)$, musculoskeletal pain ( $24 \%$ vs $15 \%)$, urinary tract infection ( $20 \%$ vs $11 \%)$, and rash ( $20 \%$ vs $2.3 \%)$. In patients with previously treated locally advanced or metastatic UC receiving BAVENCIO, the most common adverse reactions (all grades, $\geq 20 \%$ ) were fatigue, infusion-related reaction, musculoskeletal pain, nausea, decreased appetite, and urinary tract infection.

Selected laboratory abnormalities (all grades, $\geq 20 \%$ ) in patients with locally advanced or metastatic UC receiving BAVENCIO + BSC (vs BSC alone) as first-line maintenance treatment were blood triglycerides increased ( $34 \%$ vs $28 \%$ ), alkaline phosphatase increased (30\% vs $20 \%$ ), blood sodium decreased ( $28 \%$ vs $20 \%$ ), lipase increased ( $25 \%$ vs $16 \%$ ), aspartate aminotransferase (AST) increased ( $24 \%$ vs $12 \%$ ), blood potassium increased ( $24 \%$ vs $16 \%$ ), alanine aminotransferase (ALT) increased ( $24 \%$ vs $12 \%$ ), blood cholesterol increased ( $22 \%$ vs $16 \%$ ), serum amylase increased ( $21 \%$ vs $12 \%)$, hemoglobin decreased $(28 \%$ vs $18 \%)$, and white blood cell decreased ( $20 \%$ vs $10 \%)$.

Please see Brief Summary of Prescribing Information on following pages.

References: 1. Referenced with permission from the NCCN Clinical Practice Guidelines in Oncology (NCCN Guidelines ${ }^{\circledR}$ ) for Bladder Cancer V.3.2021. (C) National Comprehensive Cancer Network, Inc. 2021. All rights reserved. Accessed May 3, 2021. To view the most recent and complete version of the guidelines, go online to NCCN.org. NCCN makes no warranties of any kind whatsoever regarding their content or its use or application and disclaims any responsibility for its use or application in any way. 2. Powles T, Park SH, Voog E, et al. Avelumab maintenance therapy for advanced or metastatic urothelial carcinoma. N Engl J Med. 2020;383(13):1218-1230. 
BAVENCIO ${ }^{\circledast}$ (avelumab) injection, for intravenous use BRIEF SUMMARY: Please see package insert for Full Prescribing Information

\section{INDICATION AND USAGE}

First-Line Maintenance Treatment of Urothelial Carcinoma

BAVENCIO is indicated for the maintenance treatment of patients with locally advanced or metastatic urothelial carcinoma (UC) that has not progressed with first-line platinum-containing chemotherapy.

\section{CONTRAINDICATIONS}

\section{None.}

\section{WARNINGS AND PRECAUTIONS}

Severe and Fatal Immune-Mediated Adverse Reactions: BAVENCIO is a monoclonal antibody that belongs to a class of drugs that bind to either the programmed death-receptor 1 (PD-1) or the PD-ligand 1 (PD-L1), blocking the PD-1/PD-L1 pathway, thereby removing inhibition of the immune response, potentially breaking peripheral tolerance and inducing immune-mediated adverse reactions. Important immune-mediated adverse reactions listed under Warnings and Precautions may not include all possible severe and fatal immune-mediated reactions.

Immune-mediated adverse reactions, which may be severe or fatal, can occur in any organ system or tissue. Immune-mediated adverse reactions can occur at any time after starting treatment with a PD-1/PD-L1 blocking antibody. While immune-mediated adverse reactions usually manifest during treatment with PD-1/PD-L1 blocking antibodies, immune-mediated adverse reactions can also manifest after discontinuation of PD-1/PD-L1 blocking antibodies.

Early identification and management of immune-mediated adverse reactions are essential to ensure safe use of PD-1/PD-L1 blocking antibodies. Monitor patients closely for symptoms and signs that may be clinical manifestations of underlying immune-mediated adverse reactions. Evaluate liver enzymes, creatinine, and thyroid function at baseline and periodically during treatment. In cases of suspected immune-mediated adverse reactions, initiate appropriate workup to exclude alternative etiologies, including infection. Institute medical management promptly, including specialty consultation as appropriate.

Withhold or permanently discontinue BAVENCIO depending on severity. In general, if BAVENCIO requires interruption or discontinuation, administer systemic corticosteroid therapy (1 to $2 \mathrm{mg} / \mathrm{kg} / \mathrm{day}$ prednisone or equivalent) until improvement to Grade 1 or less. Upon improvement to Grade 1 or less, initiate corticosteroid taper and continue to taper over at least 1 month. Consider administration of other systemic immunosuppressants in patients whose immune-mediated adverse reactions ar not controlled with corticosteroid therapy. Toxicity management guidelines for adverse reactions that do not necessarily require systemic corticosteroids (e.g., endocrinopathies and dermatologic reactions) are discussed below.

Immune-Mediated Pneumonitis: BAVENCIO can cause immune-mediated pneumonitis. Immunemediated pneumonitis occurred in $1.2 \%(21 / 1738)$ of patients receiving BAVENCIO, including fatal $(0.1 \%)$, Grade $4(0.1 \%)$, Grade $3(0.3 \%)$ and Grade $2(0.6 \%)$ adverse reactions. Pneumonitis led to permanent discontinuation of BAVENCIO in $0.3 \%$ and withholding of BAVENCIO in $0.3 \%$ of patients. Systemic corticosteroids were required in all (21/21) patients with pneumonitis. Pneumonitis resolved in $57 \%(12 / 21)$ of the patients. Of the 5 patients in whom BAVENCIO was withheld for pneumonitis, 5 reinitiated treatment with BAVENCIO after symptom improvement; of these, none had recurrence of pneumonitis. With other PD-1/PD-L1 blocking antibodies, the incidence of pneumonitis is higher in patients who have received prior thoracic radiation.

Immune-Mediated Colitis: BAVENCIO can cause immune-mediated colitis. The primary component of the immune-mediated colitis consisted of diarrhea. Cytomegalovirus (CMV) infection/ reactivation has been reported in patients with corticosteroid-refractory immune-mediated colitis. In cases of corticosteroid-refractory colitis, consider repeating infectious workup to exclude alternative etiologies. Immune-mediated colitis occurred in 1.5\% (26/1738) of patients receiving BAVENCIO, including Grade $3(0.4 \%)$ and Grade $2(0.7 \%)$ adverse reactions. Colitis led to permanent discontinuation of BAVENCIO in $0.5 \%$ and withholding of BAVENCIO in $0.5 \%$ of patients. Systemic corticosteroids were required in all $(26 / 26)$ patients with colitis. Colitis resolved in $69 \%(18 / 26)$ of the patients. Of the 8 patients in whom BAVENCIO was withheld for colitis, 5 reinitiated treatment with BAVENCIO after symptom improvement; of these, $40 \%$ had recurrence of colitis.

Hepatotoxicity and Immune-Mediated Hepatitis: BAVENCIO as a single agent: BAVENCIO can cause immune-mediated hepatitis. Immune-mediated hepatitis occurred in $0.9 \%(16 / 1738)$ of patients receiving BAVENCIO, including fatal $(0.1 \%)$, Grade $3(0.6 \%)$, and Grade $2(0.1 \%)$ adverse reaction Hepatitis led to permanent discontinuation of BAVENCIO in $0.5 \%$ and withholding of BAVENCIO in $0.2 \%$ of patients. Systemic corticosteroids were required in all $(16 / 16)$ patients with hepatitis. Hepatitis resolved in $56 \%(9 / 16)$ of the patients. Of the 3 patients in whom BAVENCIO was withheld for hepatitis, 3 reinitiated treatment with BAVENCIO after symptom improvement; of these, none had recurrence of hepatitis.

Immune-Mediated Endocrinopathies: Adrenal Insufficiency: BAVENCIO can cause primary or secondary adrenal insufficiency. For Grade 2 or higher adrenal insufficiency, initiate symptomatic treatment, including hormone replacement, as clinically indicated. Withhold BAVENCIO depending on severity. Immune-mediated adrenal insufficiency occurred in $0.5 \%(8 / 1738)$ of patients receiving BAVENCIO, including Grade $3(0.1 \%)$, and Grade $2(0.3 \%)$ adverse reactions. Adrenal insufficiency led to permanent discontinuation of BAVENCIO in $0.1 \%$ and withholding of BAVENCIO in $0.1 \%$ of patients. Systemic corticosteroids were required in all $(8 / 8)$ patients with adrenal insufficiency. Adrenal insufficiency did not resolve in any patient (0/8). Of the 2 patients in whom BAVENCIO was withheld for adrenal insufficiency, none reinitiated treatment with BAVENCIO. Hypophysitis: BAVENCIO can cause immune-mediated hypophysitis. Hypophysitis can present with acute symptoms associated with mass effect such as headache, photophobia, or visual field defects. Hypophysitis can cause hypopituitarism. Initiate hormone replacement, as clinically indicated. Withhold or permanently discontinue BAVENCIO depending on severity. Immune-mediated pituitary disorders occurred in $0.1 \%(1 / 1738)$ of patients receiving BAVENCIO which was a Grade 2 $(0.1 \%)$ adverse reactions. Hypopituitarism did not lead to withholding of BAVENCIO in this patient. Systemic corticosteroids were not required in this patient. Thyroid Disorders: BAVENCIO can cause immune-mediated thyroid disorders. Thyroiditis can present with or without endocrinopathy. Hypothyroidism can follow hyperthyroidism. Initiate hormone replacement for hypothyroidism or institute medical management of hyperthyroidism, as clinically indicated. Withhold or permanently discontinue BAVENCIO depending on severity. Thyroiditis occurred in $0.2 \%(4 / 1738)$ of patients receiving BAVENCIO, including Grade $2(0.1 \%)$ adverse reactions. Thyroiditis did not lead to permanent discontinuation or withholding of BAVENCIO in any patients. No patients with thyroiditis required systemic corticosteroids. Thyroiditis did not resolve in any patients $(0 / 4)$. Hyperthyroidism occurred in $0.4 \%(7 / 1738)$ of patients receiving BAVENCIO, including Grade $2(0.3 \%)$ adverse reactions. Hyperthyroidism did not lead to permanent discontinuation of BAVENCIO in any patients and led to withholding of BAVENCIO in $0.1 \%$ of patients. Systemic corticosteroids were required in $29 \%(2 / 7)$ of patients with hyperthyroidism. Hyperthyroidism resolved in $86 \%(6 / 7)$ of the patients. Of the 2 patients in whom BAVENCIO was withheld for hyperthyroidism, 2 reinitiated treatment with BAVENCIO after symptom improvement; of these, none had recurrence of hyperthyroidism. Hypothyroidism occurred in $5 \%(90 / 1738)$ of patients receiving BAVENCIO, including Grade 3 $(0.2 \%)$ and Grade $2(3.7 \%)$ adverse reactions. Hypothyroidism led to permanent discontinuation of BAVENCIO in $0.1 \%$ and withholding of BAVENCIO in $0.5 \%$ of patients. Systemic corticosteroids were required in $7 \%(6 / 90)$ of patients with hypothyroidism. Hypothyroidism resolved in $4 \%(4 / 90)$ of the patients. Of the 8 patients in whom BAVENCIO was witheld for hypothyroidism, none reinitiated BAVENCIO. Type I Diabetes Mellitus, which can present with Diabetic Ketoacidosis: Monitor patients for hyperglycemia or other signs and symptoms of diabetes. Initiate treatment with insulin as clinically indicated. Withhold BAVENCIO depending on severity. Immune-mediated Type I diabetes mellitus occurred in $0.1 \%(2 / 1738)$ of patients receiving BAVENCIO, including
Grade $3(0.1 \%)$ adverse reactions. Type I diabetes mellitus led to permanent discontinuation of BAVENCIO in these two patients. Type I diabetes mellitus did not lead to withholding of BAVENCIO in any patient. Systemic corticosteroids were not required in any patient with Type I diabetes mellitus. Type I diabetes mellitus resolved in no patient and all patients required ongoing insulin treatment

Immune-Mediated Nephritis with Renal Dysfunction: BAVENCIO can cause immune-mediated nephritis. Immune-mediated nephritis with renal dysfunction occurred in $0.1 \%(1 / 1738)$ of patients receiving BAVENCIO, which was a Grade $2(0.1 \%)$ adverse reactions. Nephritis with renal dysfunction led to permanent discontinuation of BAVENCIO in this patient. Nephritis did not lead to withholding of BAVENCIO in any patient. Systemic corticosteroids were required in this patient. Nephritis with renal dysfunction did not resolve in this patient.

Immune-Mediated Dermatologic Adverse Reactions: BAVENCIO can cause immune-mediated rash or dermatitis. Exfoliative dermatitis, including Stevens Johnson Syndrome, DRESS, and toxic epidermal necrolysis (TEN), has occurred with PD-1/PD-L1 blocking antibodies. Topical emollients and/or topical corticosteroids may be adequate to treat mild to moderate non-exfoliative rashes. Withhold or permanently discontinue BAVENCIO depending on severity. Immune-mediated dermatologic adverse reactions occurred in $5 \%$ (90/1738) of patients receiving BAVENCIO, including Grade $3(0.1 \%)$ and Grade $2(2.0 \%)$ adverse reactions. Dermatologic adverse reactions led to permanent discontinuation of BAVENCIO in $0.3 \%$ of patients and withholding of BAVENCIO in $0.4 \%$ of patients. Systemic corticosteroids were required in $29 \%(26 / 90)$ of patients with dermatologic adverse reactions. One patient required the addition of tacrolimus to high-dose corticosteroids. Dermatologic adverse reactions resolved in $41 \%(37 / 90)$ of the patients. Of the 7 patients in whom BAVENCIO was withheld for dermatologic adverse reactions, 3 reinitiated dermatologic adverse reaction.

Other Immune-Mediated Adverse Reactions: The following clinically significant immunemediated adverse reactions occurred at an incidence of $<1 \%$ (unless otherwise noted) in patients who received BAVENCIO or were reported with the use of other PD-1/PD-L1 blocking antibodies. Severe or fatal cases have been reported for some of these adverse reactions. Cardiac/Nascular: Myocarditis, pericarditis, vasculitis. Gastrointestinal: Pancreatitis to include increases in serum amylase and lipase levels, gastritis, duodenitis. Nervous System: Meningitis, increases in serum amylase and lipase levels, gastritis, duodenitis. Nervous System: Meningitis,
encephalitis, myelitis and demyelination, myasthenic syndrome/myasthenia gravis (including exacerbation), Guillain-Barré syndrome, nerve paresis, autoimmune neuropathy. Ocular: Uveitis, iritis, and other ocular inflammatory toxicities can occur. Some cases can be associated with retinal detachment. Various grades of visual impairment, including blindness, can occur. If uveitis occurs in combination with other immune-mediated adverse reactions, consider a Vogt-KoyanagiHarada like syndrome, as this may require treatment with systemic corticosteroids to reduce the risk of permanent vision loss. Musculoskeletal and Connective Tissue: Myositis/polymyositis, rhabdomyolysis (and associated sequelae including renal failure), arthritis, polymyalgia rheumatic. Endocrine: Hypoparathyroidism. Other (Hematologic/lmmune): Hemolytic anemia, aplastic anemia, hemophagocytic lymphohistiocytosis, systemic inflammatory response syndrome, histiocytic necrotizing lymphadenitis (Kikuchi lymphadenitis), sarcoidosis, immune thrombocytopenic purpura, solid organ transplant rejection.

Infusion-Related Reactions: BAVENCIO can cause severe or life-threatening infusion-related reactions. Premedicate with antihistamine and acetaminophen prior to the first 4 infusions. Monitor patients for signs and symptoms of infusion-related reactions including pyrexia, chills, flushing, hypotension, dyspnea, wheezing, back pain, abdominal pain, and urticaria. Interrupt or slow the rate of infusion for mild or moderate infusion-related reactions. Stop the infusion and permanently discontinue BAVENCIO for severe (Grade 3) or life-threatening (Grade 4) infusion-related reactions. Infusion-related reactions occurred in $25 \%$ of patients treated with BAVENCIO including three $(0.2 \%)$ Grade 4 and nine $(0.5 \%)$ Grade 3 infusion-related reactions. Ninety-three percent of patients Grade $\geq 3$ reactions were treated with intravenous corticosteroids. Fourteen percent of patients had infusion-related reactions that occurred after the BAVENCIO infusion was completed.

Complications of Allogeneic HSCT: Fatal and other serious complications can occur in patients who receive allogeneic hematopoietic stem cell transplantation (HSCT) before or after being treated with a PD-1/PD-L1 blocking antibody. Transplant-related complications include hyperacute graft-versushost-disease (GVHD), acute GVHD, chronic GVHD, hepatic veno-occlusive disease (VOD) after reduced intensity conditioning, and steroid-requiring febrile syndrome (without an identified infectious cause). These complications may occur despite intervening therapy between PD-1/PD-L1 blockade and allogeneic HSCT. Follow patients closely for evidence of transplant-related complications and intervene promptly. Consider the benefit versus risks of treatment with a PD-1/PD-L1 blocking antibody prior to or after an allogeneic HSCT.

Embryo-Fetal Toxicity: Based on its mechanism of action, BAVENCIO can cause fetal harm when administered to a pregnant woman. Animal studies have demonstrated that inhibition of the PD-1/ PD-L1 pathway can lead to increased risk of immune-mediated rejection of the developing fetus resulting in fetal death. If this drug is used during pregnancy, or if the patient becomes pregnant while taking BAVENCIO, inform the patient of the potential risk to a fetus. Advise females of childbearing potential to use effective contraception during treatment with BAVENCIO and for at least one month after the last dose of BAVENCIO.

\section{ADVERSE REACTIONS}

The following clinically significant adverse reactions are described elsewhere in the labeling:

- Severe and fatal immune-mediated adverse reactions

- Infusion-related reactions

- Complications of allogeneic HSCT

Clinical Trials Experience: Because clinical trials are conducted under widely varying conditions, adverse reaction rates observed in the clinical trials of a drug cannot be directly compared to rates in the clinical trials of another drug and may not reflect the rates observed in practice. The data described in the WARNINGS AND PRECAUTIONS section reflect exposure to BAVENCIO $10 \mathrm{mg} /$ $\mathrm{kg}$ intravenously every 2 weeks as a single agent in 1738 patients enrolled in the JAVELIN Merkel 200 and JAVELIN Solid Tumor trials and to BAVENCIO $10 \mathrm{mg} / \mathrm{kg}$ intravenously every 2 weeks in combination with axitinib $5 \mathrm{mg}$ orally twice daily in 489 patients enrolled in the JAVELIN Renal 100 and JAVELIN Renal 101 trials. In the BAVENCIO monotherapy population, $24 \%$ of patients were exposed for $\geq 6$ months and $7 \%$ were exposed for $\geq 12$ months. The following criteria were used to classify an adverse reaction as immune-mediated: onset within 90 days after last dose of BAVENCIO, no spontaneous resolution within 7 days of onset, treatment with corticosteroids or other mmunosuppressant or hormone replacement therapy, biopsy consistent with immune-mediated reaction, and no other clear etiology.

\section{Locally Advanced or Metastatic Urothelial Carcinoma}

First-Line Maintenance Treatment of Urothelial Carcinom

The safety of BAVENCIO was evaluated in the JAVELIN Bladder 100 trial where patients received BAVENClO $10 \mathrm{mg} / \mathrm{kg}$ every 2 weeks plus best supportive care $(\mathrm{BSC})(\mathrm{N}=344)$ or $\mathrm{BSC}$ alone $(\mathrm{N}=345)$. Patients with autoimmune diseases or conditions requiring systemic immunosuppression were excluded. In the BAVENCIO plus BSC arm, $47 \%$ were exposed to BAVENCIO for $>6$ months and $28 \%$ were exposed for $>1$ year. The median age of patients treated with BAVENCIO plus BSC was 69 years (range: 37 to 90 ) $63 \%$ of patients were 65 years or older $76 \%$ were male, $67 \%$ were White, and the ECOG performance score was $0(61 \%)$ or $1(39 \%)$. A fatal adverse reaction (sepsis) occurred in one $(0.3 \%)$ patient receiving BAVENCIO plus BSC. Serious adverse reactions occurred in $28 \%$ of patients receiving BAVENCIO plus BSC. Serious adverse reactions in $\geq 1 \%$ of patients included urinary tract infection (including kidney infection, pyelonephritis, and urosepsis) (6.1\%), pain (including abdominal, back, bone, flank, extremity, and pelvic pain) (3.2\%), acute kidney injury $(1.7 \%)$, hematuria $(1.5 \%)$, sepsis $(1.2 \%)$, and infusion-related reaction $(1.2 \%)$. Permanent discontinuation due to an adverse reaction of BAVENCIO plus BSC occurred in $12 \%$ of patients. 
Adverse reactions resulting in permanent discontinuation of BAVENCIO in $>1 \%$ of patients were myocardial infarction (including acute myocardial infarction and troponin T increased) $(1.5 \%)$ and infusion-related reaction (1.2\%). Dose interruptions due to an adverse reaction, excluding temporary interruptions of BAVENCIO infusions due to infusion-related reactions, occurred in $41 \%$ of patients receiving $\mathrm{BAVENCIO}$ plus $\mathrm{BSC}$. Adverse reactions leading to interruption of $\mathrm{BAVENCIO}$ in $>2 \%$ of patients were urinary tract infection (including pyelonephritis) $(4.7 \%)$ and blood creatinine increased (including acute kidney injury, renal impairment, and renal failure) (3.8\%). The most common adverse reactions $(\geq 20 \%)$ in patients receiving BAVENCIO plus BSC were fatigue, musculoskeletal pain, urinary tract infection, and rash. Thirty-one (9\%) patients treated with BAVENCIO plus BSC received an oral prednisone dose equivalent to $\geq 40 \mathrm{mg}$ daily for an immune-mediated adverse reaction. Table 5 summarizes adverse reactions that occurred in $\geq 10 \%$ of patients treated with BAVENCIO plus BSC.

Table 5: Adverse Reactions $(\geq 10 \%$ ) of Patients Receiving BAVENCIO plus BSC (JAVELIN Bladder 100 Trial)

\begin{tabular}{|c|c|c|c|c|}
\hline \multirow{2}{*}{ Adverse Reactions } & \multicolumn{2}{|c|}{$\begin{array}{c}\text { BAVENCIO plus BSC } \\
(\mathrm{N}=344)\end{array}$} & \multicolumn{2}{|c|}{$\begin{array}{c}\text { BSC } \\
(\mathrm{N}=345)\end{array}$} \\
\hline & $\begin{array}{c}\text { All Grades } \\
\%\end{array}$ & $\begin{array}{c}\text { Grade } 3-4 \\
\%\end{array}$ & $\begin{array}{c}\text { All Grades } \\
\%\end{array}$ & $\begin{array}{c}\text { Grade } 3-4 \\
\%\end{array}$ \\
\hline \multicolumn{5}{|c|}{ General Disorders and Administration Site Conditions } \\
\hline Fatigue $^{\mathrm{a}}$ & 35 & 1.7 & 13 & 1.7 \\
\hline Pyrexia & 15 & 0.3 & 3.5 & 0 \\
\hline \multicolumn{5}{|c|}{ Musculoskeletal and Connective Tissue Disorders } \\
\hline Musculoskeletal pain ${ }^{b}$ & 24 & 1.2 & 15 & 2.6 \\
\hline Arthralgia & 16 & 0.6 & 6 & 0 \\
\hline \multicolumn{5}{|c|}{ Skin and Subcutaneous Tissue Disorders } \\
\hline Rash $^{c}$ & 20 & 1.2 & 2.3 & 0 \\
\hline Pruritus & 17 & 0.3 & 1.7 & 0 \\
\hline \multicolumn{5}{|c|}{ Infections and Infestations } \\
\hline Urinary tract infection $^{d}$ & 20 & 6 & 11 & 3.8 \\
\hline \multicolumn{5}{|c|}{ Gastrointestinal Disorders } \\
\hline Diarrhea & 17 & 0.6 & 4.9 & 0.3 \\
\hline Constipation & 16 & 0.6 & 9.0 & 0 \\
\hline Nausea & 16 & 0.3 & 6 & 0.6 \\
\hline Vomiting & 13 & 1.2 & 3.5 & 0.6 \\
\hline \multicolumn{5}{|c|}{ Respiratory, Thoracic and Mediastinal Disorders } \\
\hline Coughe & 14 & 0.3 & 4.6 & 0 \\
\hline \multicolumn{5}{|c|}{ Metabolism and Nutrition Disorders } \\
\hline Decreased appetite & 14 & 0.3 & 7 & 0.6 \\
\hline \multicolumn{5}{|l|}{ Endocrine disorders } \\
\hline Hypothyroidism & 12 & 0.3 & 0.6 & 0 \\
\hline \multicolumn{5}{|c|}{ Injury, Poisoning and Procedural Complications } \\
\hline Infusion-related reaction & 10 & 0.9 & 0 & 0 \\
\hline
\end{tabular}

${ }^{\mathrm{a}}$ Fatigue is a composite term that includes fatigue, asthenia and malaise.

${ }^{b}$ Musculoskeletal pain is a composite term that includes musculoskeletal pain, back pain, myalgia, and neck pain.

"Rash is a composite term that includes rash, rash maculo-papular, erythema, dermatitis acneiform, eczema, erythema multiforme, rash erythematous, rash macular, rash papular, rash pruritic, drug eruption and lichen planus.

dUrinary tract infection is a composite term that includes urinary tract infection, urosepsis, cystitis, kidney infection, pyuria, pyelonephritis, bacteriuria, pyelonephritis acute, urinary tract infection bacterial, and Escherichia urinary tract infection.

${ }^{e}$ Cough is a composite term that includes cough and productive cough

Patients received pre-medication with an anti-histamine and acetaminophen prior to each infusion Infusion-related reactions occurred in 10\% (Grade 3: $0.9 \%$ ) of patients treated with BAVENCIO plus BSC.

Table 6: Selected Laboratory Abnormalities Worsening from Baseline Occurring in $\geq 10 \%$ of Patients Receiving BAVENCIO plus BSC (JAVELIN Bladder 100 Trial)

\begin{tabular}{|c|c|c|c|c|}
\hline \multirow[b]{2}{*}{ Laboratory Abnormality } & \multicolumn{2}{|c|}{ BAVENCIO plus BSC* } & \multicolumn{2}{|c|}{ BSC $^{*}$} \\
\hline & $\begin{array}{c}\text { Any Grade } \\
\%\end{array}$ & $\begin{array}{c}\text { Grade } 3-4 \\
\% \\
\end{array}$ & $\begin{array}{c}\text { Any Grade } \\
\%\end{array}$ & $\begin{array}{c}\text { Grade } 3-4 \\
\%\end{array}$ \\
\hline \multicolumn{5}{|l|}{ Chemistry } \\
\hline Blood triglycerides increased & 34 & 2.1 & 28 & 1.2 \\
\hline $\begin{array}{l}\text { Alkaline phosphatase } \\
\text { increased }\end{array}$ & 30 & 2.9 & 20 & 2.3 \\
\hline Blood sodium decreased & 28 & 6 & 20 & 2.6 \\
\hline Lipase increased & 25 & 8 & 16 & 6 \\
\hline $\begin{array}{l}\text { Aspartate aminotransferase } \\
\text { (AST) increased }\end{array}$ & 24 & 1.7 & 12 & 0.9 \\
\hline Blood potassium increased & 24 & 3.8 & 16 & 0.9 \\
\hline $\begin{array}{l}\text { Alanine aminotransferase } \\
\text { (ALT) increased }\end{array}$ & 24 & 2.6 & 12 & 0.6 \\
\hline $\begin{array}{l}\text { Blood cholesterol } \\
\text { increased }\end{array}$ & 22 & 1.2 & 16 & 0.3 \\
\hline Serum amylase increased & 21 & 5 & 12 & 1.8 \\
\hline CPK increased & 19 & 2.4 & 12 & 0 \\
\hline Phosphate decreased & 19 & 3.2 & 15 & 1.2 \\
\hline \multicolumn{5}{|l|}{ Hematology } \\
\hline Hemoglobin decreased & 28 & 4.4 & 18 & 3.2 \\
\hline $\begin{array}{l}\text { White blood cell } \\
\text { decreased }\end{array}$ & 20 & 0.6 & 10 & 0 \\
\hline Platelet count decreased & 18 & 0.6 & 12 & 0.3 \\
\hline
\end{tabular}

*Each test incidence is based on the number of patients who had both baseline and at least one onstudy laboratory measurement available: BAVENCIO plus BSC group (range: 339 to 344 patients) and BSC group (range: 329 to 341 patients).
Immunogenicity: As with all therapeutic proteins, there is potential for immunogenicity. The detection of antibody formation is highly dependent on the sensitivity and specificity of the assay. Additionally, the observed incidence of antibody (including neutralizing antibody) positivity in an assay may be influenced by several factors including assay methodology, sample handling, timing of sample collection, concomitant medications, and underlying disease. For these reasons, comparison of the incidence of antibodies to avelumab in the studies described below with the incidence of antibodies in other studies or to other products may be misleading. Of the 344 patients treated with BAVENCIO $10 \mathrm{mg} / \mathrm{kg}$ as an intravenous infusion every 2 weeks plus BSC, 325 were evaluable for treatment-emergent anti-drug antibodies (ADA) and $62(19.1 \%)$ tested positive in the JAVELIN Bladder 100 trial. Patients who tested positive for treatment-emergent ADA had decreased systemic BAVENCIO exposure. In exploratory analyses, the effect of ADA on the efficacy or safety could not be determined due to insufficient numbers of patients in the ADA-positive subgroup and confounding variables.

USE IN SPECIFIC POPULATIONS

Pregnancy, Risk Summary: Based on its mechanism of action, BAVENCIO can cause fetal harm when administered to a pregnant woman. There are no available data on the use of BAVENCIO in pregnant women. Animal studies have demonstrated that inhibition of the PD-1/PD-L1 pathway can lead to increased risk of immune-mediated rejection of the developing fetus resulting in fetal death. Human IgG1 immunoglobulins (IgG1) are known to cross the placenta. Therefore, BAVENCIO has the potential to be transmitted from the mother to the developing fetus. If this drug is used during pregnancy, or if the patient becomes pregnant while taking this drug, advise the patient of the potential risk to a fetus. In the U.S. general population, the estimated background risk of major birth defects and miscarriage in clinically recognized pregnancies is $2 \%$ to $4 \%$ and $15 \%$ to $20 \%$, respectively.

Data, Animal Data: Animal reproduction studies have not been conducted with BAVENCIO to evaluate its effect on reproduction and fetal development. A central function of the PD-1/PD-L1 pathway is to preserve pregnancy by maintaining maternal immune tolerance to the fetus. In murine models of pregnancy, blockade of PD-L1 signaling has been shown to disrupt tolerance to the fetus and to result in an increase in fetal loss; therefore, potential risks of administering BAVENCIO during pregnancy include increased rates of abortion or stillbirth. As reported in the literature, there were no malformations related to the blockade of PD-1/PD-L1 signaling in the offspring of these animals; however, immune-mediated disorders occurred in PD-1 and PD-L1 knockout mice. Based on its mechanism of action, fetal exposure to BAVENCIO may increase the risk of developing immunerelated disorders or altering the normal immune response.

Lactation, Risk Summary: There is no information regarding the presence of avelumab in human milk, the effects on the breastfed infant, or the effects on milk production. Since many drugs including antibodies are excreted in human milk, advise a lactating woman not to breastfeed during treatment and for at least one month after the last dose of BAVENCIO due to the potential for serious adverse reactions in breastfed infants.

Females and Males of Reproductive Potential, Contraception: Based on its mechanism of action, BAVENCIO can cause fetal harm when administered to a pregnant woman. Advise females of reproductive potential to use effective contraception during treatment with BAVENCIO and for at least 1 month after the last dose of BAVENCIO.

Pediatric Use: Safety and effectiveness of BAVENCIO have not been established in pediatric patients Geriatric Use

Locally Advanced or Metastatic Urothelial Carcinoma: Of the 344 patients randomized to BAVENCIO $10 \mathrm{mg} / \mathrm{kg}$ plus BSC in the JAVELIN Bladder 100 trial, $63 \%$ were 65 years or older and $24 \%$ were 75 years or older. No overall differences in safety or efficacy were reported between elderly patients and younger patients.

PATIENT COUNSELING INFORMATION: Advise the patient to read the FDA-approved patient labeling (Medication Guide)

Immune-Mediated Adverse Reactions: Inform patients of the risk of immune-mediated adverse reactions requiring corticosteroids or hormone replacement therapy, including, but not limited to:

- Pneumonitis: Advise patients to contact their healthcare provider immediately for new or worsening cough, chest pain, or shortness of breath.

- Colitis: Advise patients to contact their healthcare provider immediately for diarrhea or severe abdominal pain.

- Hepatitis: Advise patients to contact their healthcare provider immediately for jaundice, severe nausea or vomiting, pain on the right side of abdomen, lethargy, or easy bruising or bleeding.

- Endocrinopathies: Advise patients to contact their healthcare provider immediately for signs or symptoms of adrenal insufficiency, hypothyroidism, hyperthyroidism, and diabetes mellitus.

- Nephritis with Renal Dysfunction: Advise patients to contact their healthcare provider immediately for signs or symptoms of nephritis including decreased urine output, blood in urine, swelling in ankles, loss of appetite, and any other symptoms of renal dysfunction.

- Dermatologic Adverse Reactions: Advise patients to contact their healthcare provider immediately for signs or symptoms of skin rash, itchy skin, rash with tiny spots and bumps, reddening of skin, blisters or peeling.

Infusion-Related Reactions: Advise patients to contact their healthcare provider immediately for signs or symptoms of potential infusion-related reactions.

Complications of Allogeneic HSCT: Advise patients of the risk of post-allogeneic hematopoietic stem cell transplantation complication

Embryo-Fetal Toxicity: Advise females of reproductive potential that BAVENCIO can cause fetal harm. Instruct females of reproductive potential to use effective contraception during and for at least one month after the last dose of BAVENCIO.

Lactation: Advise nursing mothers not to breastfeed while taking BAVENCIO and for at least one month after the final dose.

Manufactured by: EMD Serono, Inc. Rockland, MA 02370 U.S.A. US License No: 1773 Marketed by: EMD Serono, Inc. and Pfizer Inc. BAVENCIO is a trademark of Merck KGaA, Darmstadt, Germany

February 2021 US-AVE-00577

Copyright $\odot 2021$ EMD Serono, Inc. All rights reserved. 


\section{CONTINUING MEDICAL EDUCATION (CME)}

\section{The Gut Microbiome and Immunotherapy}

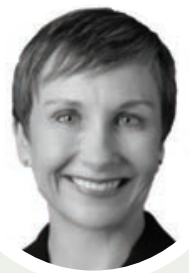

FACULTY

Jennifer McQuade, MD, MS, MA, LAc Assistant Professor

Department of Melanoma Medical Oncology

The University of Texas MD Anderson Cancer Center

Houston, Texas

This activity was written by $P E R^{\circledR}$ editorial staff under faculty guidance and review. The Q\&A portion of the activity was transcribed from a recorded interview with the faculty and edited by faculty and $\mathrm{PER}^{\circledR}$ editorial staff for clarity.

\section{CME PROVIDER CONTACT} INFORMATION

Physicians' Education Resource ${ }^{\circledR}$, LLC 2 Clarke Drive, Suite 110

Cranbury, NJ 08512

Toll-Free: 888-949-0045

Local: 609-378-3701

Fax: 609-257-0705

info@gotoper.com

\section{LEARNING OBJECTIVES}

Upon successful completion of this activity, you should be better prepared to:

- Describe the associations between the gut microbiome and immunotherapy

- Assess key data from ongoing clinical trials evaluating gut microbiome biomarkers for the use of immunotherapy

- Outline potential strategies to modulate the gut microbiome to improve efficacy of immunotherapy

RELEASE DATE: July 1,2021

EXPIRATION DATE: July 1, 2022

\section{INSTRUCTIONS FOR PARTICIPATION / HOW TO RECEIVE CREDIT}

\section{Read this activity in its entirety.}

2. Go to https://www.gotoper.com/go/onc-gut2021immuno to access and complete the posttest.

3. Answer the evaluation questions.

4. Request credit using the drop-down menu.

You may immediately download your certificate.

FACULTY, STAFF, AND PLANNERS' DISCLOSURES

In accordance with ACCME Guidelines, PER ${ }^{\circledR}$ has identified and resolved all COl for faculty, staff, and planners prior to the start of this activity by using a multistep process.

Disclosures (Dr McQuade): Consultant: Merck

The staff of PER ${ }^{\circledast}$ have no relevant financial relationships with commercial interests to disclose.

\section{OFF-LABEL DISCLOSURE AND DISCLAIMER}

This activity may or may not discuss investigational, unapproved, or off-label use of drugs. Learners are advised to consult prescribing information for any products discussed. The information provided in this activity is for accredited continuing education purposes only and is not meant to substitute for the independent clinical judgment of a healthcare professional relative to diagnostic, treatment, or management option for a specific patient's medical condition. The opinions expressed in the content are solely those of the individual faculty members, and do not reflect those of PER ${ }^{\circledR}$ or any of the companies that provided commercial support for this activity.

This activity is funded by $\mathrm{PER}{ }^{\circledR}$.

\section{ACCREDITATION/CREDIT DESIGNATION}

Physicians' Education Resource ${ }^{\circledast}$, LLC, is accredited by the Accreditation Council for Continuing Medical Education (ACCME) to provide continuing medical education for physicians. Physicians' Education Resource ${ }^{\circledR}$, LLC, designates this enduring material for a maximum of 0.5 AMA PRA Category 1 Credits $^{\text {tm. }}$. Physicians should claim only the credit commensurate with the extent of their participation in the activity. 


\section{ACTIVITY}

The emergence of cancer immunotherapy has brought hope to many patients with cancer. Cancer immunotherapy blocks immune checkpoints, thereby reactivating immune function and restoring the anti-tumor activity of T cells. ${ }^{1}$ The most widely used immune checkpoint inhibitors (ICIs) are monoclonal antibodies that target the programmed cell death protein 1 (PD-1), its ligand (PD-L1), or the cytotoxic T-lymphocyte antigen 4 protein (CTLA-4). Immune checkpoint inhibitors are highly effective for a subset of patients, but only 10 to $60 \%$ of all tumors respond to treatment. ${ }^{2,3}$ A critical need remains to identify predictors of response and resistance and to develop synergistic strategies to increase response to treatment. ${ }^{4}$

Multiple studies indicate that gut microbes, a diverse community of more than 100 trillion microorganisms residing in the intestines, can profoundly influence outcomes with immunotherapy. ${ }^{5,6}$ Initial evidence for the contribution of specific microbes in response to anti-CTLA-4 and anti-PD-1/PD-L1 was first demonstrated in mouse models. Results from multiple institutions have since contributed to the growing consensus that the gut microbiome is linked to immunotherapy efficacy in patients with cancer. ${ }^{5}$ To date, these studies implicate a range of bacteria in facilitating a response or non-response to ICIs in patients with melanoma. ${ }^{2}$ Immune-related toxicity of ICIs has also been linked to the composition of the gut microbiome. ${ }^{5}$

These findings open up the exciting possibility that efficacy of ICIs may be improved by manipulating the gut flora. Potential intervention strategies that have been suggested range from less precise to more targeted therapeutic approaches.
These include fecal microbiota transplantation (FMT), the use of prebiotics/ diet, probiotics, genetically modified bacteria, and selective depletion of harmful species from the microbiota. ${ }^{5}$

The hope is that manipulating the intestinal microbiota could enhance the effectiveness of ICIs and reduce adverse effects, thereby leading to new breakthroughs in immunotherapy and cancer management. ${ }^{1}$

Jennifer McQuade, MD, MS, MA, LAc, reviews recent advances in modulation of the gut microbiome to enhance response to immunotherapy.

\section{What are the major overarching physiologic mechanisms by which the gut microbiome influences the immune system in healthy individuals?} MCQUADE: There is an intimate and symbiotic relationship between microorganisms and their hosts, with whom they coevolved. ${ }^{7}$ We know from germ-free mouse studies that the microbiome is actually critical in training our immune system during early life and these early exposures influence our susceptibility to infection and disease throughout our life. The gut microbiota is the most well-studied microbial "niche". There, the microbiota-immune interaction occurs at the mucosal barrier. We need to maintain this compartmentalization and for our immune system to recognize and eliminate potential pathogenic bacteria if that barrier is broken. However, our immune system also needs to tolerate the good bacteria that are helping us break down food and extract nutrients. ${ }^{8}$

\section{In what ways could microbiome dysregulation contribute to oncogenesis and tumor progression?} MCQUADE: This has been most well studied in the context of GI luminal diseases, for example colorectal cancer and gastric cancer. We know that H. pylori, a pathogenic bacterium in our stomach, is implicated in ulcerative disease as well as in gastric cancer. ${ }^{9}$ With colorectal cancer there's actually been quite a bit of work around pathogenic bacteria leading to oncogenesis. And there most of the focus has been on a particular bacterium called fusobacterium. ${ }^{10}$

The role the gut microbiome may play in non-luminal GI malignancies is something that a lot of people are actively looking at. But there's not great data, with the exception of something that connects a bit with the lumen; pancreatic cancer. What we see there is that you can actually have retrograde movement of some of these bacteria actually into the pancreas. You don't necessarily see these pathogenic bacteria within the healthy pancreas but you do within the diseased pancreas. There have actually been some FMT studies in mice showing that if you modify the gut bacteria, then you can modify the pancreatic tumor microbiome as well, and then change progression of disease and course of disease, as well as influence response to chemotherapy. ${ }^{11,12}$ There is also now increasing evidence that non-luminal cancers have a distinct microbiome.

\section{In what ways could the gut microbiome affect the effectiveness of immune checkpoint inhibitors?}

MCQUADE: If we want to think about immune checkpoint inhibitors, we should probably back up a little bit first and think about the original immunotherapies. The first data around the gut microbiome and response to any form of immunotherapy was really in stem cell transplants. We know that in patients with AML - who of course are patients who tend to be quite ill, they have often received broad-spectrum antibiotics, their nutritional status might be impaired, et cetera-the gut microbiome is associated with outcomes in stem cell transplants. ${ }^{13}$ There, the microbiome has been associated with risk of graft-versus-host 
disease and transplant-related mortality. ${ }^{14}$ There was then some work led by Laurence Zitvogel showing that cyclophosphamide, which is a chemotherapy drug that has a heavy reliance on immune function for its anticancer activity, is dependent on the gut microbiome. ${ }^{15}$.

As far as with checkpoint inhibitors, data first emerged in two papers that were published in 2015 looking at the role of the gut microbiome in response to checkpoint inhibitors in mice. ${ }^{16,17}$ These were from Tom Gajewski's group in Chicago and Laurence Zitvogel in France, and the story behind this is actually really interesting. When we do mouse experiments, we buy our mice from a couple of large vendors here in the US. We inject the mice with cancer and then give them anticancer treatment. If you give chemotherapy, it really doesn't matter which lab you get the mice from with regard to how they respond to treatment, as they are genetically identical mice, just from different vendors.

However, investigators studying immunotherapy noted that the tumors injected into mice that were purchased from one vendor would not respond to immunotherapy, whereas the tumors of the mice purchased from the other vendor, would. So they said, "These are genetically identical mice, so why would this be?" It had to be environmental. And that's what led them to look at the microbiome, which is largely determined by our exposures/ environment. And, sure enough, they profiled the gut microbiomes and saw different bugs in the mice that came from one vendor compared to the other. This, of course, doesn't prove that this was the cause of the difference in immune responsiveness. They, therefore, then did FMTs from one vendor's mice to the other and found they could basically transmit responsiveness to immunotherapy via gut microbiome modulation. ${ }^{18}$

So that was really interesting. But those are mice in a highly controlled environment that doesn't really represent the complexity of the human microbiome

\section{aci}

"In cancer patients with immunotherapy, fecal microbiota transplantation is safe, it can modulate the microbiome, and there is enough promise of immune activation to lead to larger studies."

or of our environmental exposures. The question then became, "Is this actually relevant in humans?" So Tom Gajewski, Laurence Zitvogel, and then Jennifer Wargo at MD Anderson all started profiling the gut microbiota of humans who were starting immune checkpoint blockade. Each of their groups identified specific bacterial signatures that were associated with response versus non-response to immunotherapy. ${ }^{19-21}$

Now this is, of course, correlation and not causation. So they then took this back into the mouse model. They did experiments where they took fecal material from the humans who responded to immunotherapy and from the humans who did not respond. They then did an FMT from the humans into the mice, and then injected the mice with cancer and treated them with immunotherapy. And what they saw was that in those mice who received the responder FMT, their tumors would then respond to immunotherapy. And those mice who received the non-responder FMT, theirs would not. ${ }^{22}$ This was a really important proof of principle of (1) looking at causality, but (2) also looking at the idea: can we actually use microbiome modulation to impact response to immune checkpoint blockade?

\section{What are the key strategies to modulating the gut microbiome?}

MCQUADE: There are a number of different ways to modulate the microbiome. FMT is obviously the most direct and has the advantage that we don't actually have to completely understand which bacteria or groups of bacteria are potentially beneficial as we are giving them all. In all of these human cohort studies, specific bacteria have been associated with response, but the microbiome is a very complex ecosystem. Is it really that specific bacteria, or is that bacteria just a bellwether? Or is it not just one particular type of bacteria but a clade of bacteria; a little unit of 10 or 20 different bacteria that are working together? Or is it not actually about the particular bacteria, but what the bacteria are doing, for example, producing short-chain fatty acid metabolites? So with FMT, you don't have to know what the magic ingredient is; you give them everything. ${ }^{4}$

The other way to modulate the microbiome would be with probiotics. ${ }^{5}$ Probiotics are basically putatively beneficial bacteria. Commercially available probiotics are basically aerobic bacteria that are easy to culture. But if we think about it, $90 \%$ of the bacteria that live in our gut are actually anaerobic bacteria. The bacteria that are identified as associated with response to immunotherapy are predominantly anaerobic bacteria. And so commercially available probiotics are really not necessarily what the gut needs but what are easy to culture and grow and make into pills. ${ }^{23}$ There have been some interesting studies, too, where when you give commercially available probiotics you're actually crowding out some of the beneficial bacteria. There was a really beautiful study from Israel that was published in Cell where these bacteria are actually actively secreting something that's inhibiting the growth of other bacteria. ${ }^{24}$ So nonselective probiotics could be potentially harmful by 
disrupting this ecosystem.

Next-generation probiotics are something that people are now thinking about. As we become more sophisticated with anaerobically culturing these bacteria and then identifying not just one kind of bacteria but maybe a group of bacteria that work together, we can even do some assays where we take these groups of bacteria outside of the body and then see what those can do as far as stimulating T-cell function. There are a number of clinical trials right now that are looking at these next-generation, multistrain probiotics that are really rationally designed specifically within this context of immune checkpoint blockade.

Other strategies include the use of prebiotics. Instead of giving us the good bacteria, can we instead provide the food sources that would support the growth and outgrowth of these bacteria? ${ }^{5}$ Now, the prototypical prebiotic is fiber, and a lot of the bacteria that have been associated with response are actually bacteria that have known roles in fiber fermentation. When these bacteria ferment fiber, they produce these short-chain fatty acid metabolites that are actually the primary nutrient source for our gut enterocytes. They improve the mucosal barrier, and there's also some evidence now that there can be direct epigenetic modulation of T-cell activity to increase memory $\mathrm{T}$ cells. So there are also ongoing studies looking at high-fiber diet to modulate the microbiome in cancer patients. ${ }^{25}$

\section{What are the current major} challenges in modulating gut microbiome to improve response to immunotherapy in patients with cancer? MCQUADE: Each of these things has their own challenges. For FMT, the issue is scal- ability. FMT is a specialized and invasive procedure that is not widely available. Many of the early trials have used patients who responded to immunotherapy as donors, but that has proven to be very challenging in terms of donor identification and recruitment. So are "healthy donors" just as good? We are also still working to define who the most appropriate recipients would be. Should it be in the treatment-naive setting or in the treatment-refractory setting? Should it be based on baseline profiling showing a "bad" microbiome? But do we really know enough at this point to distinguish what is a good vs a bad microbiome?

Diet, on the other hand, is accessible to everyone. And we know that a high-fiber diet with fruits, vegetables, and whole grains also decreases our risk of cardiovascular disease, stroke and secondary cancer. The issue here is that diet change is really hard. We know that these things are really good for secondary cancer prevention, we know that they're good for cardiovascular disease, but a lot of us still don't do those things. And so it's important to support people through these changes. Also, is it a big enough instrument to really influence response to immunotherapy? What is the dose of diet change required? How radically do you have to shift the diet to really make that change apparent in the microbiome and immunity?

In terms of probiotics, we are back to the issue of do we really know which bacteria are the critical ones for stimulating anti-tumor immunity or is it an entire clan? Will it be a one-size-fits-all solution or can we selectively modulate individuals' microbiomes?

So, accessibility is the issue for FMT while for probiotics, it's really identify- ing what the beneficial ones are. And then for diet it's the fact that behavior change is hard. But the other real thing is we're still learning. We went from the associations to starting to look at causality, and the third phase that we're in right now is starting to understand mechanism. We know if we change the microbiome, we can change immunity, but we don't know exactly how we're doing it quite yet.

\section{. What are the key data from clinical trials evaluating gut microbiome modulating strategies for patients receiving cancer immunotherapy?}

MCQUADE: There were actually just 2 papers that were published in Science. The first: pilot studies, phase 1 studies looking at FMT plus PD-1 immunotherapy in patients with melanoma that were previously refractory to immunotherapy. One study is from the Israeli group looking at 10 patients, ${ }^{26}$ and then another one is from the University of Pittsburgh looking at 15 patients. ${ }^{27}$

These early data give us the hint that this is a promising strategy. I do think, however, that we have to be mindful of the fact that these were phase 1 studies, and the endpoint of phase 1 studies is safety, not efficacy. So was this safe? Yes. The patients got an FMT, either via colonoscopy or via fecal pills. And they saw no increase in any rates of adverse events beyond what you would expect to see with just immunotherapy alone. So, is it safe to be doing FMT in cancer patients actively receiving immunotherapy? These data suggest that it is.

The second question that was really relevant is can we modulate the microbiome? We have known for a long time with Clostridium difficile that we can do an FMT from a healthy donor and very reliably shift the gut microbiome, basi-

Need even more continuing medical education? Join us online at www.gotoPER.com for more available CME courses! 
cally making the recipient microbiome look like the donor microbiome. It is an excellent treatment for treatment-refractory $C$ diff. ${ }^{28}$

Now, $C$ diff is a profoundly dysbiotic state. The entire normal gut flora has been totally wiped out, not only by the overgrowth of this pathogenic bacteria, but also by multiple rounds of the antibiotics that led to the $C$ diff, followed by multiple courses of antibiotics to try to get rid of $C$ diff, hospitalizations, et cetera. That one is an easy one to shift the microbiome in.

There have been lots of studies looking at FMT in diseases that are not as dysbiotic, for example, inflammatory bowel disease, which is relative dysbiosis but not as severe as $C$ diff. ${ }^{29}$ They found there that a lot of times it's not enough to just do one single FMT; you need these fecal pills with repeated microdoses to be able to sustain this. And there is also the question of whether you should use antibiotics beforehand to ablate the microbiome and wipe it out. But what if we wipe it out and we cause harm and then we're not able to really reestablish it? So these 2 studies were really important because they showed that there was a shift in the microbiome, and we did see the recipient microbiomes become like the donor microbiome.

Now, beyond the safety of microbiome modulation, we all, of course, also want to know if there is a suggestion of efficacy. Both of these studies enrolled patients with immunotherapy treatment-refractory melanoma. Typically, if we try to rechallenge with immunotherapy, we would expect about $15 \%$ response rate. Here, we're seeing a response rate more like $30 \%$, which is promising, but again you can't be sure about a response rate from a 10 -person study. But what was interesting is that both of these studies also looked at immune markers as well. ${ }^{26,27}$ This goes along with responding to immunotherapy, but after FMT we went from a cold microenvironment to a hot microenvironment with T-cell infil- trates and activation of all of these markers. They've seen changes in the mucosal immunity as well. I think these are really important early proof-of-principle studies that in cancer patients with immunotherapy, FMT is safe. FMT can modulate the microbiome in this setting, and there is enough promise of immune activation to lead to larger studies. But then we come back to the scalability issues.

\section{Do you have any concluding thoughts?}

MCQUADE: I would say that there's now a really strong body of evidence that the gut microbiome can indeed influence response to immunotherapy. Where we are now is improving our understanding of the mechanism and then being able to design scalable interventions to modulate the microbiome and identify patients who would benefit.

\section{KEY REFERENCES}

4. McQuade JL, Ologun GO, Arora R, Wargo JA. Gut microbiome modulation via fecal microbiota transplant to augment immunotherapy in patients with melanoma or other cancers. Curr Oncol Rep. 2020;22(7):74. doi:10.1007/ s11912-020-00913-y

5. Fessler J, Matson V, Gajewski TF. Exploring the emerging role of the microbiome in cancer immunotherapy. $J$ Immunother Cancer. 2019;7(1):108. doi:10.1186/s40425019-0574-4

19. Derosa L, Routy B, Kroemer G, Zitvogel L. The intestinal microbiota determines the clinical efficacy of immune checkpoint blockers targeting PD-1/PD-L1. Oncoimmunology. 2018;7(6):e1434468. doi:10.1080/2162402X.2018.1434468

20. Matson V, Fessler J, Bao R, et al. The commensal microbiome is associated with anti-PD-1 efficacy in metastatic melanoma patients. Science. 2018;359(6371):104108. doi:10.1126/science.aao3290

21. Gopalakrishnan V, Spencer CN, Nezi L, et al. Gut microbiome modulates response to anti-PD-1 immunotherapy in melanoma patients. Science. 2018;359(6371):97-103. doi: 10.1126/science.aan4236

26. Baruch EN, Youngster I, Ben-Betzalel G, et al. Fecal microbiota transplant promotes response in immunotherapy-refractory melanoma patients. Science. 2021;371(6529): 602-609. doi:10.1126/science.abb5920

27. Davar D, Dzutsev AK, McCulloch JA, et al. Fecal microbiota transplant overcomes resistance to anti-PD-1 therapy in melanoma patients. Science. 2021;371(6529):595-602. doi:10.1126/science.abf3363

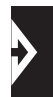

For full reference list, visit https://www.gotoper.com/go/ onc-gut2021immuno

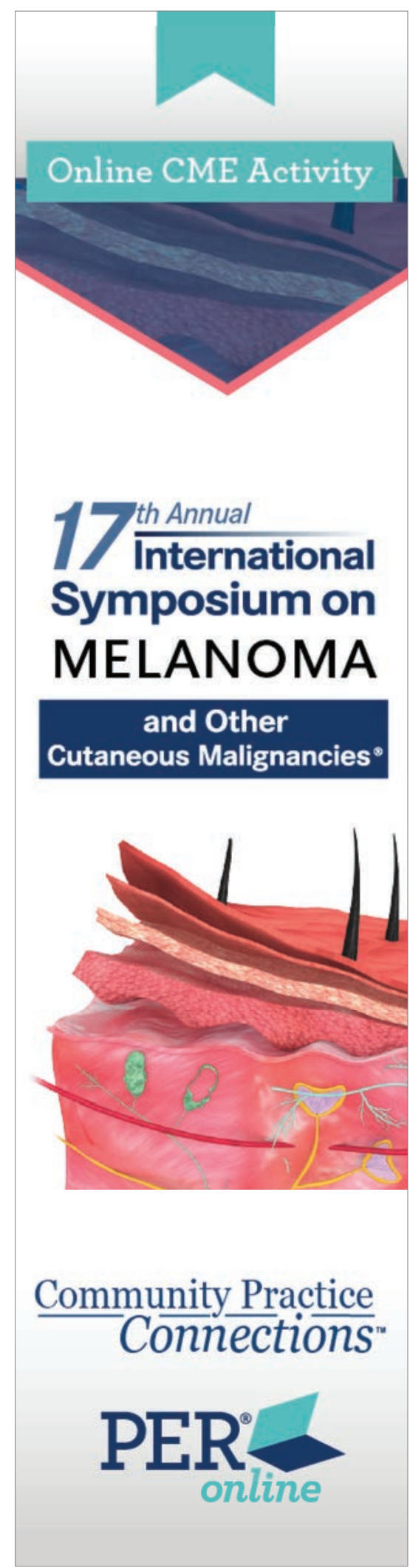




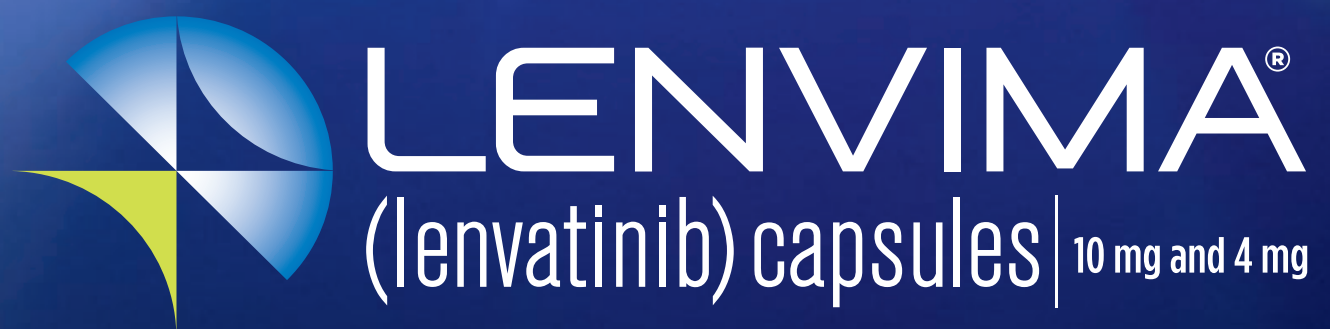

\section{(lenvatinib) capsules| 10 mgand ams}

\section{To learn more, visit www.LENVIMA.com/hcp}

